\title{
Álgebras de Koszul e resoluções projetivas
}

Francisco Batista de Medeiros

\author{
DisSERTAÇÃO APRESENTADA \\ $\mathrm{AO}$ \\ Instituto De Matemática E Estatística \\ DA \\ Universidade de São Paulo \\ PARA \\ OBTENÇÃO DO TÍTULO \\ $\mathrm{DE}$ \\ Mestre Em CiÊnCIAS
}

\author{
Programa: Matemática \\ Orientador: Prof. Dr. Eduardo do Nascimento Marcos
}

Durante o desenvolvimento deste trabalho o autor recebeu auxílio financeiro do CNPq

São Paulo, julho de 2009 


\title{
Álgebras de Koszul e resoluções projetivas
}

\author{
Este exemplar corresponde à redação \\ final da dissertação devidamente corrigida \\ e defendida por Francisco Batista de Medeiros \\ e aprovada pela Comissão Julgadora.
}

Banca Examinadora:

- Prof. Dr. Eduardo do Nascimento Marcos (orientador) - IME-USP.

- Prof. Dr. Héctor Alfredo Merklen Goldschmidt - IME-USP.

- Prof. Dr. Wagner de Oliveira Cortes - UFRGS. 


\section{Resumo}

Neste trabalho estudamos algumas características das álgebras de Koszul, como por exemplo, a maneira como elas se relacionam com suas respectivas álgebras de Yoneda. Descrevemos a álgebra de Yoneda de uma álgebra monomial e como aplicação construímos uma família de álgebras: as chamadas homologicamente auto-duais. Uma álgebra de Koszul pode ser definida a partir da existência de resoluções lineares dos módulos simples. Por isso faz-se necessário a dedicação de parte de nossa atenção ao estudo destas resoluções. Além disso, achamos interessante estudar métodos para a construção de resoluções projetivas de módulos sobre quocientes de álgebras de caminhos. Para tal construção usamos essencialmente a teoria de bases de Gröbner não comutativas. Finalmente, para o caso de módulos lineares sobre álgebras de Koszul, veremos que é possível modificar essa construção de modo que a resolução resultante seja linear.

Palavras-chave: álgebras de Koszul, resoluções projetivas, resoluções lineares, bases de Gröbner, álgebra de extensões. 


\section{Abstract}

In this work we study some features of Koszul algebras as, for example, the way that they are related with their Yoneda algebras. We describe the Yoneda algebra of a monomial algebra and as an application we construct a family of algebras: the so called homologically self-dual algebras. A Koszul algebra can be defined as an algebra for which there are linear resolutions of their simple modules. Because of this we dedicate part of our attention to the study of projective resolutions. The study of methods for the construction of projectives resolutions of modules over quotients of path algebras, has an of interest its own. For the study of projective resolutions we used the theory of noncommutative, Gröbner bases. Finally, for the case of linear modules over Koszul algebras, we will see that it is possible to modify the general construction described here, so that the resulting resolution is linear.

Keywords: Koszul algebras, projetive resolutions, linear resolutions, Gröbner bases, algebra of extensions. 


\section{Sumário}

Introdução vii

1 Preliminares $\quad 1$

1.1 Categorias e Funtores . . . . . . . . . . . . . . . . . . . . . 1

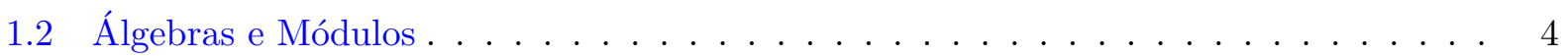

1.3 Produto tensorial de módulos . . . . . . . . . . . . . . . . . 11

1.4 Álgebra de Caminhos . . . . . . . . . . . . . . . . . . . . . . 13

1.5 Álgebras Graduadas . . . . . . . . . . . . . . . . . . . . 16

2 Álgebras de Yoneda $\quad 21$

2.1 Introdução . . . . . . . . . . . . . . . . . . . . . . . . 21

2.2 Álgebra de Yoneda de uma álgebra monomial . . . . . . . . . . . . . . . . . . 23

2.3 Carcás de $E(\Lambda) \ldots \ldots \ldots \ldots \ldots \ldots \ldots \ldots$

2.4 Uma construção de álgebras homologicamente auto-duais . . . . . . . . . . . . . 29

3 Álgebras de Koszul $\quad 33$

3.1 Resoluções Lineares . . . . . . . . . . . . . . . . . . . . . . . . 33

3.2 Álgebras de Koszul e suas álgebras de Yoneda . . . . . . . . . . . . . . . . . . . 35

3.3 Álgebras de Koszul e álgebras quadráticas . . . . . . . . . . . . . . . . . . . 41

3.4 Álgebra de Koszul vista como $\Lambda^{e}$-módulo . . . . . . . . . . . . . . . . . . 44

4 Resoluções Projetivas $\quad 47$

4.1 Introdução . . . . . . . . . . . . . . . . . . . 47

4.2 Exemplo de módulos com base ordenada . . . . . . . . . . . . . . . . . . 55

4.3 Construindo uma resolução projetiva . . . . . . . . . . . . . . . . 60

4.4 Resoluções finitamente geradas . . . . . . . . . . . . . . . . . . 75

4.5 Resoluções de módulos lineares sobre álgebras de Koszul . . . . . . . . . . . . . . . 79

$\begin{array}{lr}\text { Considerações Finais } & 87\end{array}$

$\begin{array}{ll}\text { Referências Bibliográficas } & 89\end{array}$

$\begin{array}{ll}\text { Índice Remissivo } & 91\end{array}$ 


\section{Introdução}

As álgebras de Koszul foram introduzidas por Priddy ${ }^{1}$ em 1970. Desde 1970, muito se estudou a respeito desta classe de álgebras graduadas. Elas desempenham um papel importante no estudo de Álgebras Comutativas e na Topologia Algébrica. Na década de 90, apareceram artigos com aplicações importantes de álgebras de Koszul a topologia algébrica, Teoria de Lie e Grupos Quânticos, como pode ser visto em [7,10,23,24].

Uma álgebra graduada gerada em graus 0 e 1 é chamada álgebra de Koszul se cada módulo simples admite um resolução linear, ou seja, uma resolução projetiva na qual:

- o projetivo $P_{i}$ que ocupa a $i$-ésima posição na resolução é um módulo graduado gerado em grau $i$.

- cada $d_{i}: P_{i} \rightarrow P_{i-1}$ é um morfismo de grau zero, ou seja, a imagem da componente $\left(P_{i}\right)_{j}$ de grau $j$ de $P_{i}$ está contida na componente $\left(P_{i-1}\right)_{j}$ de grau $j$ de $P_{i-1}$.

Começamos o trabalho com um estudo da álgebra de Yoneda $E(\Lambda)$, também conhecida como álgebra de extensões, de uma álgebra $\Lambda$ dada, pois resoluções lineares possuem uma relação estreita com módulos graduados sobre a álgebra de Yoneda, como pode ser vista no seguinte teorema:

Teorema. Sejam $\Lambda=\bigoplus_{n \geq 0} \Lambda_{n}$ uma álgebra graduada 0,1-split-básica e $M$ um $\Lambda$-módulo graduado gerado em grau 0. Então as seguintes afirmações são equivalentes:

1. $M$ possui uma resolução linear.

2. $E(M)$ é um $E(\Lambda)$-módulo graduado gerado em grau 1.

Uma k-álgebra graduada $\Lambda=\bigoplus_{n \geq 0} \Lambda_{n}$ é chamada 0,1 -split-básica quando é gerada em graus zero e um, $\Lambda_{0}$ é um produto direto de cópias de k e $\Lambda_{1}$ é um k-espaço vetorial de dimensão finita.

Num primeiro momento, nos restrigimos às álgebras monomiais, ou seja, álgebras da forma $\Lambda=\mathrm{k} \Gamma / I$, onde $\mathrm{k}$ é um corpo, $\Gamma$ um carcás finito e $I$ um ideal de $\mathrm{k} \Gamma$ gerado por monômios. Uma das vantagens de considerar esse caso é a existência de uma k-base multiplicativa para $E(\Lambda)$, a qual pode ser descrita por subconjuntos ${ }^{2}$ do carcás $\Gamma$. A partir de um subconjunto minimal desta k-base, que ainda gere $E(\Lambda)$ como álgebra, será possível construir um carcás $\Delta$ e uma apresentação

\footnotetext{
${ }^{1}$ Veja $[25]$

${ }^{2}$ Conhecidos na literatura por conjuntos de cadeias ou de "overlaps".
} 
$\psi$ para $E(\Lambda)$ de modo que $E(\Lambda)$ seja isomorfa a $\mathrm{k} \Delta /$ Nuc $(\psi)$ e os elementos do núcleo de $\psi$ ficam bem determinados a partir do conjunto minimal escolhido acima. A partir dessa apresentação, Green e Zacharia em [20] mostram que a álgebra de Yoneda de uma álgebra quadrática monomial é ainda uma álgebra monomial, é finitamente gerada e seus geradores estão em grau no máximo 1. Isso implicará que as álgebras quadráticas monomiais são exemplos de álgebras de Koszul. Além disso, como aplicação desses conceitos, fizemos um breve estudo das álgebras homologicamente auto-duais, que são aquelas isomorfas a sua álgebra de extensões. Em [20], os autores perguntam se todas as álgebras (monomiais) homologicamente auto-duais têm a forma descrita no texto e no Capítulo 2 exibimos contra-exemplos para esta questão.

Num segundo momento, de forma menos específica, passamos a procurar outros exemplos de álgebras de Koszul, além das quadráticas monomiais. Fazendo uso de uma fórmula de Butler concluiremos que as álgebras de Koszul são quadráticas e que as quadráticas com dimensão global igual a 2 também são exemplos de álgebras de Koszul.

Um resultado importante que relaciona álgebras de Koszul com sua álgebra de extensões é o seguinte:

Teorema. Seja $\Lambda$ uma álgebra 0,1-split-básica. Então $\Lambda$ é uma álgebra de Koszul se, e somente se, $E(\Lambda)$ é gerada em graus 0 e 1 .

Uma consequência desse resultado é que uma álgebra graduada 0,1-split-básica é Koszul se, e somente se, o carcás de $E(\Lambda)$ coincide com o carcás de $\Lambda$. Veremos também que quando a álgebra de Yoneda da álgebra de Yoneda de $\Lambda$ for isomorfa a $\Lambda$, como álgebra graduada, então $\Lambda$ é uma álgebra de Koszul.

Finalmente, daremos uma caracterização de álgebras de Koszul através de sua álgebra envolvente.

Vimos aqui que as álgebras de Koszul estão diretamente relacionadas com a existência de resoluções lineares para os módulos simples. Por isso, achamos conveniente estudar métodos para construção de resoluções projetivas de módulos sobre quocientes de álgebras de caminhos e ver em que situações tal construção pode ser transformada em uma resolução linear. Tal construção é feita para módulos sobre álgebras que dadas como quocientes de álgebras de caminhos, uma vez que toda álgebra graduada 0,1-split-básica é isomorfa a uma deste tipo. Para a construção de resoluções projetivas, Green e Solberg em [13] fazem uso da teoria de Bases de Gröbner não comutativas, uma ferramenta importante e comum em outras áreas, como Álgebra Computacional e Geometria Algébrica. Essa resolução nem sempre é minimal, mas é sempre algorítimica. Um dos resultados interessantes é o seguinte teorema de [13]:

Teorema. Se $I$ é um ideal que possui base de Gröbner finita e $M$ é um $\mathrm{k} \Gamma / I$-módulo (à direita) que é finitamente apresentado como $\mathrm{k} \Gamma$-módulo, então podemos construir uma $\mathrm{k} \Gamma / I$-resolução projetiva para $M$ onde cada projetivo é finitamente gerado. 
Anteriormente, em [14], Green, Solberg e Zacharia demonstraram um resultado análogo, supondo porém que $\mathrm{k} \Gamma / I$ é uma álgebra noetheriana, $I$ um $\mathrm{k} \Gamma$-módulo finitamente gerado e $M$ um $\mathrm{k} \Gamma / I$ módulo finitamente gerado.

Um caso particular de qualquer um desses resultados, é quando $I$ é um ideal admissível e $M$ é $\mathrm{k} \Gamma / I$-módulo finitamente gerado.

É importante salientar que independentemente disso, o estudo de resoluções projetivas se justificaria por outras razões, como por exemplo, é uma ferramenta de suma importância para o tratamento de propriedades homológicas de anéis e módulos. Além disso, é bem conhecido que tais resoluções ocupam espaço dentro da teoria de anéis comutativos e teoria de Representação de Álgebras de dimensão finita.

\section{Organização do Trabalho}

No Capítulo 1, fazemos um resumo com definições e resultados gerais envolvendo teoria de categorias, módulos sobre álgebras, produto tensorial e álgebras graduadas. Tais conceitos serão utilizados livremente no decorrer do texto. A bibliografia utilizada foi $[1,2,8,21,22]$.

No Capítulo 2 estudamos a estrutura da álgebra de extensões, também chamada álgebra de Yoneda, de uma álgebra monomial. O estudo é baseado basicamente no fato, apresentado em [20], de que a álgebra de Yoneda de uma álgebra monomial possui uma base multiplicativa. O principal resultado mostra que a álgebra de extensões de uma álgebra monomial quadrática é finitamente gerada e todos os seus geradores estão gerados em grau no máximo 1.

Já o Capítulo 3 é dedicado ao estudo da álgebra de Yoneda de uma álgebra graduada. Veremos que a existência de resoluções projetivas (lineares) dos módulos simples sobre uma álgebra graduada $\Lambda$, está estreitamente ligada ao comportamento da sua álgebra de extensões $E(\Lambda)$. Mostramos, por exemplo, que se cada módulo simples sobre $\Lambda$ têm resolução linear, então $E(\Lambda)$ é gerada em grau no máximo 1, e vice-versa. O desenvolvimento deste capítulo segue basicamente [18,26]. Usaremos também alguns resultados de [19] e de [4].

Finalmente, no Capítulo 4 apresentamos um procedimento para a construção de resoluções projetivas de módulos sobre quocientes de álgebras de caminhos, isto é, álgebras da forma $\Lambda=\mathrm{k} \Gamma / I$, para algum ideal $I$ contido em $J^{2}$, onde $J$ denota o ideal de $\mathrm{k} \Gamma$ gerado por todas as flechas de $\Gamma$. Veremos o que podemos exigir do módulo em questão e do ideal $I$ para que este procedimento produza uma resolução projetiva onde cada termo é um $\Lambda$-módulo finitamente gerado. Faremos também, em certas condições, ajustes na construção para que a resolução obtida seja linear. A ferramenta fundamental neste estudo será a teoria de Bases de Gröbner. No desenrolar do capítulo, seguimos o que foi feito em $[13,14]$ e fazemos uso de $[5,11,12]$. 


\section{Capítulo 1}

\section{Preliminares}

Começamos nosso trabalho incluindo as definições e os resultados básicos que serão utilizados nos próximos capítulos. A maior parte dos resultados terão suas demonstrações omitidas, as quais podem ser encontradas, por exemplo, em [1,2,8,22]. Em algumas partes do trabalho utilizamos propriedades de categorias e funtores, por isso incluímos inicialmente uma seção com algumas definições e resultados. Estes podem ser encontrados por exemplo em $[1,8]$.

\subsection{Categorias e Funtores}

Uma categoria $\mathcal{C}$ é definida por:

- uma classe de objetos de $\mathcal{C}$, denotada por Ob $\mathcal{C}$;

- para cada par $(X, Y)$ de objetos de $\mathcal{C}$ associamos um conjunto chamado de conjunto de morfismos de $X$ para $Y$, denotado por $\operatorname{Hom}_{\mathcal{C}}(X, Y)$, tal que se $(X, Y) \neq\left(X^{\prime}, Y^{\prime}\right)$ então $\operatorname{Hom}_{\mathcal{C}}(X, Y) \cap \operatorname{Hom}_{\mathcal{C}}\left(X^{\prime}, Y^{\prime}\right)=\emptyset$;

- para cada tripla $(X, Y, Z)$ de objetos de $\mathcal{C}$, há uma operação de composição de morfismos, denotada por o: $\operatorname{Hom}_{\mathcal{C}}(Y, Z) \times \operatorname{Hom}_{\mathcal{C}}(X, Y) \rightarrow \operatorname{Hom}_{\mathcal{C}}(X, Z)$ tal que

$-h \circ(g \circ f)=(h \circ g) \circ f$ para todo $f \in \operatorname{Hom}_{\mathcal{C}}(X, Y), g \in \operatorname{Hom}_{\mathcal{C}}(Y, Z), h \in \operatorname{Hom}_{\mathcal{C}}(Z, W)$;

- para todo objeto $X$ de $\mathcal{C}$ existe um morfismo $i d_{X}$ em $\operatorname{Hom}_{\mathcal{C}}(X, X)$, chamado de morfismo identidade de $X$, tal que $f \circ i d_{X}=f$ e $i d_{X} \circ g=g$, para todo morfismo $f \in \operatorname{Hom}_{\mathcal{C}}(X, Y)$ e $g \in \operatorname{Hom}_{\mathcal{C}}(Z, X)$.

Escreveremos, por abuso de notação, $X \in \mathrm{Ob} \mathcal{C}$, ou ainda $X \in \mathcal{C}$ para dizer que $X$ é um objeto da categoria $\mathcal{C}$ e $f: X \rightarrow Y$ (ou $X \stackrel{f}{\rightarrow} Y$ ) para denotar um morfismo em $\operatorname{Hom}_{\mathcal{C}}(X, Y)$. Além disso, na maioria dos casos também escreveremos $f g$ no lugar de $f \circ g$.

Dizemos que um morfismo $f: X \rightarrow Y$ é um epimorfismo que cinde se existe um morfismo $g: Y \rightarrow X$ tal que $f g=i d_{Y}$, e que é um monomorfismo que cinde se existe um morfismo $h: Y \rightarrow X$ tal que $h f=i d_{X}$. Diremos que o morfismo $f: X \rightarrow Y$ é um isomorfismo se ele é um epimorfismo que cinde e um monomorfismo que cinde, ou seja, se existir $h: Y \rightarrow X$ tal que $h f=i d_{X}$ e $f h=i d_{Y}$. Nesse último caso, dizemos que os objetos $X$ e $Y$ são isomorfos e escrevemos $X \cong Y$. 
Dada uma família de objetos $\left\{X_{\sigma}\right\}_{\sigma}$ de $\mathcal{C}$, a soma direta é um objeto de $\mathcal{C}$, denotado por $\bigoplus_{\sigma} X_{\sigma}$, junto com uma família de morfismos $u_{\sigma}: X_{\sigma} \rightarrow \bigoplus_{\sigma} X_{\sigma}$, tais que para cada objeto $Z \in$ Ob $\mathcal{C}$ e cada conjunto de morfismos $f_{\sigma}: X_{\sigma} \rightarrow Z$, em $\mathcal{C}$, existe um único morfismo $f: \bigoplus_{\sigma} X_{\sigma} \rightarrow Z$ tal que para cada $\sigma$ vale $f_{\sigma}=f \circ u_{\sigma}$. Cada morfismo $u_{\sigma}$ é chamado da $\sigma$-ésima inclusão.

Uma categoria $\mathcal{C}$ é dita aditiva se:

- para quaisquer objetos $X_{1}, \ldots, X_{n}$ de $\mathcal{C}$ existe a soma direta $X_{1} \oplus \ldots \oplus X_{n}$ em $\mathcal{C}$;

- o conjunto $\operatorname{Hom}_{\mathcal{C}}(X, Y)$ tem estrutura de grupo abeliano, para cada $X, Y \in \mathcal{C}$;

- existe um objeto zero, $0 \in \mathrm{Ob} \mathcal{C}$, tal que o morfismo identidade $i d_{0}$ é o elemento nulo do grupo abeliano $\operatorname{Hom}_{\mathcal{C}}(0,0)$;

- para $f, g, h$ morfismos em $\mathcal{C}$, vale $(f+g) \circ h=f \circ h+g \circ h$ e $f \circ(g+h)=f \circ g+f \circ h$ (desde que estas operações estejam definidas), ou seja, a composição é bilinear.

Para uma categoria $\mathcal{C}$, a categoria dual ou oposta, denotada por $\mathcal{C}^{o p}$ é definida como a categoria cujos objetos são os mesmos de $\mathcal{C}, \operatorname{Hom}_{\mathcal{C}^{o p}}(X, Y)=\operatorname{Hom}_{\mathcal{C}}(Y, X)$ para $X, Y \in \operatorname{Ob} \mathcal{C}$ e a composição de $f \in \operatorname{Hom}_{\mathcal{C}^{o p}}(X, Y) \operatorname{com} g \in \operatorname{Hom}_{\mathcal{C}^{o p}}(Y, Z)$ é $f g \in \operatorname{Hom}_{\mathcal{C}}(Z, X)=\operatorname{Hom}_{\mathcal{C}^{o p}}(X, Z)$.

Dizemos que uma categoria $\mathcal{D}$ é uma subcategoria de $\mathcal{C}$ se:

- a classe $\mathrm{Ob} \mathcal{D}$ é uma subclasse de $\mathrm{Ob} \mathcal{C}$;

- se $X, Y \in \mathrm{Ob} \mathcal{D}$, então $\operatorname{Hom}_{\mathcal{D}}(X, Y) \subseteq \operatorname{Hom}_{\mathcal{C}}(X, Y)$;

- a composição de $\mathcal{D}$ é a restrição da composição em $\mathcal{C}$;

- para cada objeto $X$ de $\mathcal{D}$, o morfismo identidade em $\operatorname{Hom}_{\mathcal{D}}(X, X)$ coincide com o morfismo identidade em $\operatorname{Hom}_{\mathcal{C}}(X, X)$.

Uma subcategoria $\mathcal{D}$ de $\mathcal{C}$ é dita plena se $\operatorname{Hom}_{\mathcal{D}}(X, Y)=\operatorname{Hom}_{\mathcal{C}}(X, Y)$ para todos os objetos $X$ e $Y$ em $\mathcal{D}$.

\section{Funtores}

Sejam $\mathcal{C}$ e $\mathcal{D}$ categorias, um funtor covariante $F: \mathcal{C} \rightarrow \mathcal{D}$ consiste de dois ingredientes, associase para cada objeto $X$ de $\mathcal{C}$ um objeto $F X$ (ou $F(X)$ ) de $\mathcal{D}$ e para cada morfismo $f: X \rightarrow Y$ em $\mathcal{C}$, um morfismo $F f: F X \rightarrow F Y$ (ou $F(f))$ em $\mathcal{D}$ tal que:

- $F(g f)=(F g)(F f)$, para todos $f$ e $g$ morfismos em $\mathcal{C}$;

- $F i d_{X}=i d_{F X}$, para todo objeto $X$ de $\mathcal{C}$.

Define-se um funtor contravariante $F: \mathcal{C} \rightarrow \mathcal{D}$ associando para cada objeto $X$ de $\mathcal{C}$ um objeto $F X$ de $\mathcal{D}$ e para cada morfismo $f: X \rightarrow Y$ em $\mathcal{C}$, um morfismo $F f: F Y \rightarrow F X$ em $\mathcal{D}$ tal que:

- $F(g f)=(F f)(F g)$, para todos $f$ e $g$ morfismos em $\mathcal{C}$; 
- $F i d_{X}=i d_{F X}$, para todo objeto $X$ de $\mathcal{C}$.

Observe que um funtor contravariante $F: \mathcal{C} \rightarrow \mathcal{D}$ é o "mesmo" que um funtor covariante $F: \mathcal{C} \rightarrow \mathcal{D}^{o p}$

Sejam $\mathcal{C}$ e $\mathcal{D}$ categorias aditivas. Um funtor $F: \mathcal{C} \rightarrow \mathcal{D}$ é dito aditivo se $F: \operatorname{Hom}_{\mathcal{C}}(X, Y) \rightarrow$ $\operatorname{Hom}_{\mathcal{D}}(F X, F Y)$ é um homomorfismo de grupos abelianos, para todos $X$ e $Y$ em $\mathcal{C}$.

Sejam $F$ e $G$ funtores covariantes da categoria $\mathcal{C}$ na categoria $\mathcal{D}$. Um morfismo funtorial ou transformação natural $\Phi: F \rightarrow G$ é uma família $\left\{\Phi_{X}\right\}_{X \in O b \mathcal{C}}$ de morfismos $\Phi_{X}: F X \rightarrow G X$ de $\mathcal{D}$ tal que, se $f: X \rightarrow Y$ é um morfismo de $\mathcal{C}$, então $\Phi_{Y} \circ F f=G f \circ \Phi_{X}$, ou seja, o seguinte diagrama é comutativo:

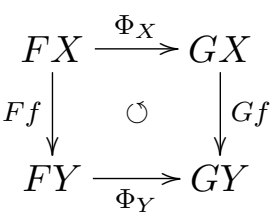

Sejam $F$ e $G$ funtores contravariantes da categoria $\mathcal{C}$ na categoria $\mathcal{D}$. Um morfismo funtorial ou transformação natural $\Phi: F \rightarrow G$ é uma família $\left\{\Phi_{X}\right\}_{X \in O b \mathcal{C}}$ de morfismos $\Phi_{X}: F X \rightarrow G X$ de $\mathcal{D}$ tal que, se $f: X \rightarrow Y$ é um morfismo de $\mathcal{C}$, então $\Phi_{X} \circ F f=G f \circ \Phi_{Y}$, ou seja, o seguinte diagrama é comutativo:

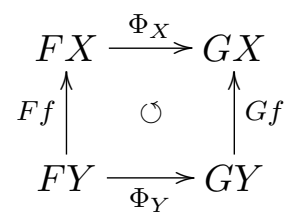

A composição de morfismos funtoriais definida por $(\Phi \Psi)_{X}=\Phi_{X} \Psi_{X}$ é ainda um morfismo funtorial. Para cada funtor $F$ tem-se um morfismo funtorial identidade $i d_{F}: F \rightarrow F$ dado por $\left(i d_{F}\right)_{X}=i d_{F X}$. Um morfismo funtorial $\Phi: F \rightarrow G$ é dito isomorfismo funtorial se cada $\Phi_{X}$ é um isomorfismo em $\mathcal{D}$, nesse caso existe um morfismo funtorial $\Psi: G \rightarrow F$ tal que $\Phi \Psi=i d_{G}$ e $\Psi \Phi=i d_{F}$. Utilizaremos $F \approx G$ para dizer que existe um isomorfismo funtorial entre $F$ e $G$. A composição de isomorfismos funtoriais é também um isomorfismo funtorial.

Um funtor (covariante) $F: \mathcal{C} \rightarrow \mathcal{D}$ é dito uma equivalência entre as categorias $\mathcal{C}$ e $\mathcal{D}$ se existir um funtor $G: \mathcal{D} \rightarrow \mathcal{C}$ tal que $F G \approx i d_{\mathcal{D}}$ e $G F \approx i d_{\mathcal{C}}$. Nesse caso, dizemos que $G$ é um quaseinverso de $F$ e que as categorias $\mathcal{C}$ e $\mathcal{D}$ são equivalentes $(\mathcal{C} \approx \mathcal{D})$. Um funtor contravariante $F: \mathcal{C} \rightarrow \mathcal{D}$ é dito uma dualidade entre as categorias $\mathcal{C}$ e $\mathcal{D}$ se o funtor covariante induzido $\dot{F}^{\prime}: \mathcal{C}^{o p} \rightarrow \mathcal{D}$ for uma equivalência de categorias.

Um funtor covariante $F: \mathcal{C} \rightarrow \mathcal{D}$ induz, para cada par $(X, Y)$ de objetos de $\mathcal{C}$, uma aplicação $F: \operatorname{Hom}_{\mathcal{C}}(X, Y) \rightarrow \operatorname{Hom}_{\mathcal{D}}(F X, F Y)$ dada por $f \mapsto F f$. Se esta aplicação é injetora, dizemos que o funtor $F$ é fiel. Se for sobrejetora, dizemos que o funtor $F$ é pleno. O funtor $F$ é dito denso se para cada objeto $M$ de $\mathcal{D}$ existir um objeto $X$ de $\mathcal{C}$ tal que $M$ e $F X$ são isomorfos em $\mathcal{D}$, isto é, existem morfismos $M \stackrel{f}{\rightarrow} F X$ e $F X \stackrel{g}{\rightarrow} M$ em $\mathcal{D}$ com $f g=i d_{F X}$ e $g f=i d_{M}$.

A seguinte proposição nos dá uma boa caracterização de uma esquivalência de categorias, e esta é de suma importância. 
Proposição 1.1 Sejam $\mathcal{C}$ e $\mathcal{D}$ categorias. Um funtor $F: \mathcal{C} \rightarrow \mathcal{D}$ é uma equivalência de categorias se, e somente se, $F$ é fiel, pleno e denso.

\section{2 Álgebras e Módulos}

Seja k um anel associativo, comutativo e com unidade 1. Uma k-álgebra $\Lambda$ é um anel com unidade (diferente de zero) que ao mesmo tempo é um módulo sobre $\mathrm{k}$ e tal que para todo $x \in \mathrm{k}$ e para todo $a, b \in \Lambda$ vale:

$$
(a b) x=a(b x)=(a x) b .
$$

Pode-se formalizar essa noção dizendo que $\Lambda$ é uma k-álgebra se existe um homomorfismo de anéis $\mathrm{k} \rightarrow \operatorname{centro}(\Lambda)$.

A toda k-álgebra $\Lambda$ associamos uma outra k-álgebra, chamada de álgebra oposta $\Lambda^{o p}$ que tem a mesma estrutura de k-módulo de $\Lambda$, mas a multiplicação $*$ é definida por $a * a^{\prime}:=a^{\prime} a$ para todos $a, a^{\prime} \in \Lambda$.

Um ideal à esquerda (à direita) $I$ da k-álgebra $\Lambda$ é um ideal à esquerda do anel $\Lambda$, ou seja, $I$ é um subgrupo aditivo de $\Lambda$ e $a x \in I(x a \in I)$, para todos $a \in \Lambda$ e $x \in I$. Quando $I$ é um ideal à esquerda e à direita dizemos que $I$ é um ideal bilateral, ou simplesmente um ideal.

Um ideal (à esquerda ou à direita) $I$ é dito nilpotente se existe um número natural $n \geq 1$ tal que $I^{n}=0$.

Exemplo 1.1 Consideremos $I$ um ideal de uma k-álgebra $\Lambda$. Seja $\Lambda / I$ o conjunto das classes módulo $I$ da forma $a+I=\{a+\alpha: \alpha \in I\}$, para todo $a \in \Lambda$. Então, $\Lambda / I$ tem uma estrutura de k-módulo dada por $(a+I)+(b+I):=(a+b)+I$ e $(a+I) x:=a x+I$, para todo $a, b \in \Lambda$ e para todo $x \in \mathrm{k}$. Note que $\Lambda / I$ tem uma estrutura de anel dada por $(a+I)(b+I):=a b+I$, para todo $a, b \in \Lambda$. Além disso, essas duas estruturas são compatíveis, ou seja, para todos $a, b \in \Lambda$ e para todo $x \in \mathrm{k}:((a+I)(b+I)) x=(a+I)((b+I) x)=((a+I) x)(b+I)$. Logo, $\Lambda / I$ é uma k-álgebra, $a$ qual chamamos de álgebra quociente de $\Lambda$ por $I$.

Seja $I$ um ideal à direita da k-álgebra $\Lambda$. Um subconjunto $\rho \subseteq I$ é dito um gerador do ideal $I$ se $I$ o é menor ideal à direita contendo $\rho$. Ou equivalentemente, se cada elemento $\omega$ de $I$ possa ser escrito como $\omega=\sum_{i=1}^{n} \omega_{i} a_{i}$ onde $\omega_{i}=\prod_{j=1}^{t} \rho_{j}$ e cada $\rho_{j} \in \rho$. Se existir algum conjunto gerador finito, $I$ é dito finitamente gerado.

Um ideal $I$ próprio de uma k-álgebra $\Lambda$ é dito maximal se não existir um ideal $\bar{I}$ de $\Lambda$ tal que $I \subsetneq \bar{I} \subsetneq \Lambda$.

Definição 1.1 Seja $\Lambda$ uma k-álgebra. A interseção de todos os ideais maximais de $\Lambda$ é chamado de radical (de Jacobson) de $\Lambda$ e é denotado por $\underline{\mathrm{r}}$.

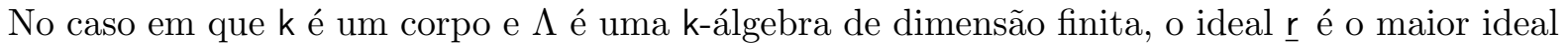
nilpotente de $\Lambda$. 


\section{Morfismos de álgebras}

Sejam $\Lambda$ e $\Sigma$ duas k-álgebras. Um morfismo ou homomorfismo de k-álgebras de $\Lambda$ em $\Sigma$ é uma aplicação $\phi: \Lambda \rightarrow \Sigma$ que é k-linear e é um homomorfismo de anéis. Dizemos que as álgebras $\Lambda$ e $\Sigma$ são isomorfas e denotamos por $\Lambda \cong \Sigma$ se existe um morfismo de k-álgebras $\phi: \Lambda \rightarrow \Sigma$ que é uma bijeção.

Seja $\phi: \Lambda \rightarrow \Sigma$ um morfismo de álgebras. Então:

1. A imagem de $\phi(\operatorname{Im} \phi:=\{\phi(a) \mid a \in \Lambda\})$ é uma subálgebra de $\Sigma$;

2. $\phi(0)=0$ e $\phi(-a)=-\phi(a)$

3. O núcleo de $\phi$ ( Nuc $\phi:=\{a \in \Lambda \mid \phi(a)=0\}$ ) é um ideal bilateral de $\Lambda$;

4. $\phi$ é injetora se, e somente se, Nuc $\phi=0$.

Proposição 1.2 (Teorema do isomorfismo) Se $\phi: \Lambda \rightarrow \Sigma$ é um homomorfismo de álgebras, então

$$
\frac{\Lambda}{\operatorname{Nuc} \phi} \cong \operatorname{Im} \phi
$$

\section{Módulos}

Seja $\Lambda$ uma k-álgebra. Um $\Lambda$-módulo à direita $M$ é simplesmente um módulo à direita sobre o anel $\Lambda$. Neste caso, $M$ é dotado de uma estrutura natural de k-módulo à direita, dada por $m x:=m\left(1_{\Lambda} x\right)$, para todo $m \in M$ e para todo $x \in \mathrm{k}$. Como k é comutativo, $M$ também tem estrutura de k-módulo à esquerda. Além disso, se $x \in \mathrm{k}, m \in M$ e $a \in \Lambda$ vale

$$
(x m) a=x(m a)=m(x a) .
$$

Usaremos a seguinte notação: $M_{\Lambda}$ denota um $\Lambda$-módulo à direita.

Um subconjunto $X \subseteq M_{\Lambda}$ é um conjunto gerador de $M$ se $M$ é o menor $\Lambda$-módulo à direita contendo $X$. Ou equivalentemente, se cada elemento $m \in M$ pode ser escrito na forma $\sum_{i=1}^{n} m_{i} a_{i}$, para alguns $m_{i} \in X$ e alguns $a_{i} \in \Lambda$. Se existir um conjunto gerador finito, $M$ é dito finitamente gerado.

Exemplo 1.2 Sejam $M_{\Lambda}$ um $\Lambda$-módulo e I um ideal de $\Lambda$, então o conjunto

$$
M I:=\left\{\sum_{j} m_{j} \alpha_{j} \mid m_{j} \in M, \alpha_{j} \in I\right\}
$$

é um $\Lambda$-submódulo de $M_{\Lambda}$.

Se $M$ for anulado pelo ideal $I$, isto é, se $M I=0$, então $M$ possui uma estrutura natural de $\Lambda / I$-módulo, dada por $m \cdot(a+I):=$ ma, para $m \in M$ e $a \in \Lambda$. 
Sejam k um corpo e $\Lambda$ uma k-álgebra de dimensão finita e $M$ é um $\Lambda$-módulo à direita. $\mathrm{O}$ radical de $M$, denotado por $\operatorname{rad} M$, é o $\Lambda$-submódulo $M \underline{r}$. O radical do $\Lambda$-módulo $\Lambda_{\Lambda}$ é $\operatorname{rad} \Lambda_{\Lambda}=\Lambda \underline{r}=\underline{r}$. Vale ainda que $\operatorname{rad}(M \oplus N)=\operatorname{rad} M \oplus \operatorname{rad} N$; e se $N_{\Lambda}$ é um submódulo de $M \operatorname{com} N \subseteq \operatorname{rad} M$, então $\operatorname{rad}\left(\frac{M}{N}\right)=\frac{\operatorname{rad} M}{N}$.

Sejam $\Lambda$ e $\Sigma$ duas k-álgebras. Um conjunto $M$ que tem estrutura de $\Lambda$-módulo à esquerda e estrutura de $\Sigma$-módulo à direita é um $(\Lambda-\Sigma)$-bimódulo se estas estruturas forem compatíveis, isto é, se $a(m b)=(a m) b$ para todo $a \in \Lambda, m \in M$ e $b \in \Sigma$. Denotaremos um $(\Lambda$ - $\Sigma)$-bimódulo $M$ por ${ }_{\Lambda} M_{\Sigma}$.

\section{Morfismos de módulos}

Sejam $M$ e $N$ dois $\Lambda$-módulos à direita. Um morfismo de $\Lambda$-módulos ou homomorfismo de $\Lambda$-módulos é uma aplicação $f: M \rightarrow N$ tal que $f\left(m_{1} a+m_{2} b\right)=f\left(m_{1}\right) a+f\left(m_{2}\right) b$ para todos $m_{1}, m_{2} \in M$ e $a, b \in \Lambda$. Um morfismo de $\Lambda$-módulos é, claramente, k-linear.

Se $f: M \rightarrow N$ é um morfismo de $\Lambda$-módulos, então $f$ é um monomorfismo se $f$ é injetora e $f$ é um epimorfismo se $f$ é sobrejetora. Se $f$ é bijetora, então é um isomorfismo de módulos, neste caso dizemos que $M$ e $N$ são isomorfos e denotamos por $M \cong N$.

Para um morfismo de módulos à direita $f: M \rightarrow N$ valem as seguintes propriedades:

1. A imagem de $f$ e o núcleo de $f$ são submódulos de $N$ e $M$ respectivamente;

2. $f(0)=0, f(-m)=-f(m)$ e $f(\operatorname{rad} M) \subseteq \operatorname{rad} N$;

3. $f$ é um monomorfismo se, e somente se, Nuc $f=0$;

4. $f$ é um epimorfismo se, e somente se, Conuc $f:=\frac{M}{\operatorname{Im} f}=0$.

Denotamos por $\operatorname{Hom}_{\Lambda}(M, N)$ o conjunto dos morfismos (de $\Lambda$-módulos) de $M$ em $N$. Tal conjunto tem estrutura de k-módulo com as seguintes operações: se $f, g \in \operatorname{Hom}_{\Lambda}(M, N)$ e $x \in \mathrm{k}$ definimos $(f+g)(m):=f(m)+g(m)$ e $(f x)(m):=f(m) x$, para todo $m \in M$.

Denotaremos por Mod $\Lambda$ ( $\Lambda$-Mod ) a categoria aditiva cujos objetos são os $\Lambda$-módulos à direita (à esquerda) e os morfismos são os morfismos de $\Lambda$-módulos e, por mod $\Lambda$ ( $\Lambda$-mod ) a subcategoria plena de Mod $\Lambda$ ( $\Lambda$-Mod ) cujos objetos são os $\Lambda$-módulos finitamente gerados.

Seja $M_{\Lambda}$ um $\Lambda$-módulo. Definimos um funtor covariante $\operatorname{Hom}_{\Lambda}(M,-): \operatorname{Mod} \Lambda \rightarrow$ Mod k que a cada $\Lambda$-módulo $N_{\Lambda}$ associa o k-módulo $\operatorname{Hom}_{\Lambda}(M, N)$ e a cada morfismo de $\Lambda$-módulos $f: L_{\Lambda} \rightarrow N_{\Lambda}$ associa o morfismo k-linear $\operatorname{Hom}_{\Lambda}(M, f): \operatorname{Hom}_{\Lambda}(M, L) \rightarrow \operatorname{Hom}_{\Lambda}(M, N)$, onde $\operatorname{Hom}_{\Lambda}(M, f)(g):=$ fg. Analogamente, definimos o funtor contravariante $\operatorname{Hom}_{\Lambda}(-, M): \operatorname{Mod} \Lambda \rightarrow$ Mod k que a cada módulo $N_{\Lambda}$ associa o k-módulo $\operatorname{Hom}_{\Lambda}(M, N)$ e a cada morfismo de $\Lambda$-módulos $f: L_{\Lambda} \rightarrow N_{\Lambda}$ associa o morfismo k-linear $\operatorname{Hom}_{\Lambda}(f, M): \operatorname{Hom}_{\Lambda}(N, M) \rightarrow \operatorname{Hom}_{\Lambda}(L, M)$, onde $\operatorname{Hom}_{\Lambda}(f, M)(g):=g f$.

Exemplo 1.3 Dado um $\Lambda$-módulo $M_{\Lambda} \in \operatorname{Mod} \Lambda$ temos que $\operatorname{Hom}_{\Lambda}\left({ }_{\Lambda} \Lambda_{\Lambda}, M_{\Lambda}\right) \cong M_{\Lambda}$. Mais ainda, esse isomorfismo é funtorial, ou seja, $\operatorname{Hom}_{\Lambda}\left(\Lambda_{\Lambda} \Lambda_{\Lambda},-\right) \approx i d_{\operatorname{Mod} \Lambda}$. 
Exemplo 1.4 (Funtor dual) Sejam k um corpo e $\Lambda$ uma k-álgebra de dimensão finita. O funtor contravariante $D:=\operatorname{Hom}_{\mathrm{k}}(-, \mathrm{k}): \bmod \Lambda \rightarrow \bmod \Lambda^{o p}$ é uma dualidade cujo funtor quase-inverso é $D:=\operatorname{Hom}_{\mathrm{k}}(-, \mathrm{k}): \bmod \Lambda^{o p} \rightarrow \bmod \Lambda$, ou seja, $D^{2}:=D \circ D \approx i d_{\bmod \Lambda}$ e portanto existe uma dualidade entre as categorias $\bmod \Lambda$ e $\bmod \Lambda^{o p}$

\section{Sequência exata de módulos}

Uma sequência de $\Lambda$-módulos e de morfismos de $\Lambda$-módulos

$$
\cdots \longrightarrow M_{i+1} \stackrel{f_{i+1}}{\longrightarrow} M_{i} \stackrel{f_{i}}{\longrightarrow} M_{i-1} \stackrel{f_{i-1}}{\longrightarrow} \cdots
$$

é dita exata em $M_{i}$ se $\operatorname{Im} f_{i+1}=$ Nuc $f_{i}$. Além disso, a sequência é dita exata se for exata em todo $M_{i}$.

Observemos que dado um morfismo de $\Lambda$-módulos $f: M \rightarrow N$, temos que $f$ é um monomorfismo se, e somente se, a sequência $0 \rightarrow M \stackrel{f}{\rightarrow} N$ for exata; e $f$ é um epimorfismo se, e somente se, a sequência $M \stackrel{f}{\rightarrow} N \rightarrow 0$ for exata.

Uma sequência exata de $\Lambda$-módulos da forma $0 \longrightarrow L \stackrel{f}{\longrightarrow} M \stackrel{g}{\longrightarrow} N \longrightarrow 0$ é chamada de sequência exata curta. Dizemos que uma sequência exata curta cinde se $f$ for um monomorfismo que cinde, ou equivalentemente, se $g$ for um epimorfismo que cinde. Nesse caso, $M \cong L \oplus N$.

Uma dualidade é sempre exata, ou seja, se $0 \rightarrow L \rightarrow M \rightarrow N \rightarrow 0$ é uma sequência exata curta, então a sequência induzida $0 \rightarrow D N \rightarrow D M \rightarrow D L \rightarrow 0$ também é exata.

Lema 1.3 (Lema da Serpente) Considere um diagrama comutativo de linhas exatas como abaixo:

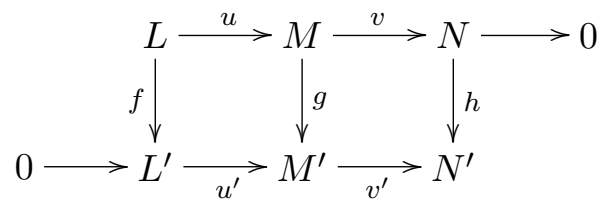

Então existe uma sequência exata da forma

$$
\operatorname{Nuc}(f) \stackrel{u_{1}}{\rightarrow} \operatorname{Nuc}(g) \rightarrow \operatorname{Nuc}(h) \stackrel{\delta}{\rightarrow} \text { Conuc }(f) \rightarrow \text { Conuc }(g) \stackrel{v_{2}}{\rightarrow} \text { Conuc }(h)
$$

Além disso,

- Se u é um monomorfismo, então u também é um monomorfismo.

- Se v' é um epimorfismo, então $v_{2}$ também é um epimorfismo.

Podemos visualizar o lema acima através do seguinte diagrama comutativo com linhas e colunas 
exatas:

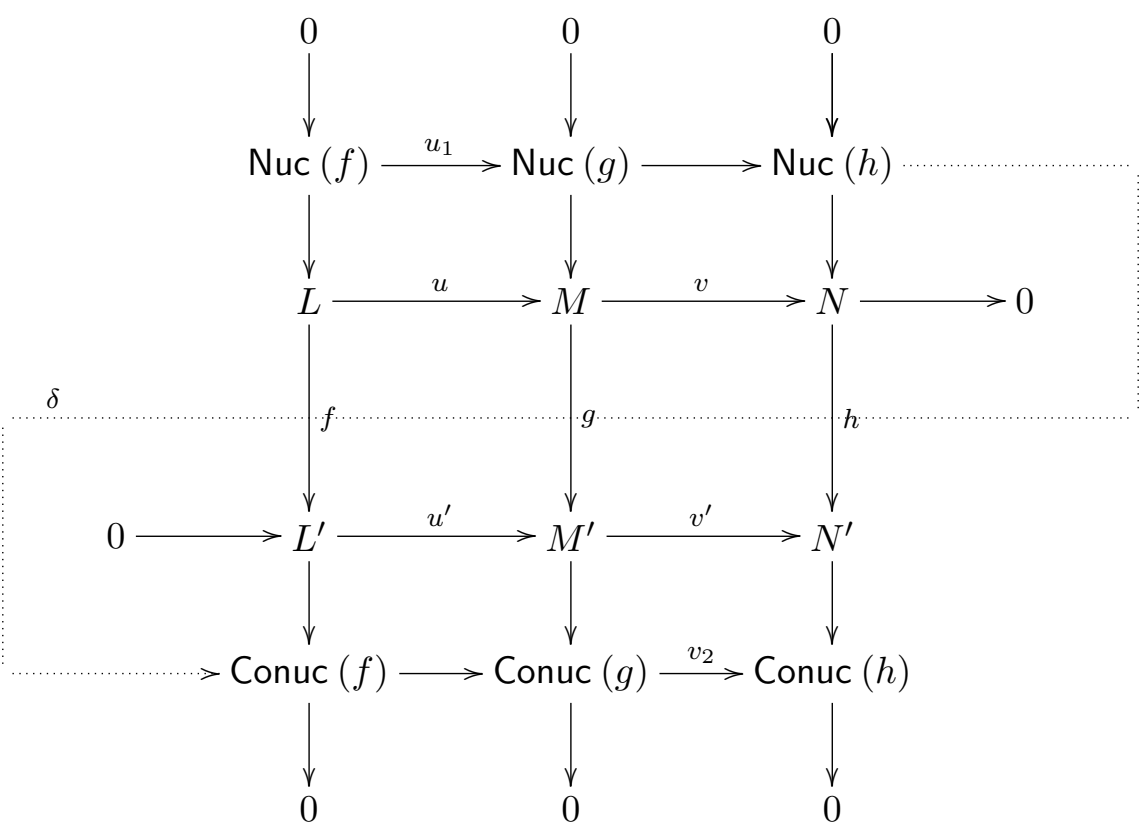

Proposição 1.4 Seja $\Lambda$ uma k-álgebra.

a) A sequência $0 \rightarrow L_{\Lambda} \stackrel{f}{\rightarrow} M_{\Lambda} \stackrel{g}{\rightarrow} N_{\Lambda}$ é exata se, e somente se, para todo $X_{\Lambda}$, a sequência de k-módulos $0 \longrightarrow \operatorname{Hom}_{\Lambda}(X, L) \stackrel{\operatorname{Hom}_{\Lambda}(X, f)}{\longrightarrow} \operatorname{Hom}_{\Lambda}(X, M) \stackrel{\operatorname{Hom}_{\Lambda}(X, g)}{\longrightarrow} \operatorname{Hom}_{\Lambda}(X, N)$ é exata.

b) A sequência $L_{\Lambda} \stackrel{f}{\rightarrow} M_{\Lambda} \stackrel{g}{\rightarrow} N_{\Lambda} \rightarrow 0$ é exata se, e somente se, para todo $X_{\Lambda}$, a sequência de k-módulos $0 \longrightarrow \operatorname{Hom}_{\Lambda}(N, X) \stackrel{\operatorname{Hom}_{\Lambda}(g, X)}{\longrightarrow} \operatorname{Hom}_{\Lambda}(M, X) \stackrel{\operatorname{Hom}_{\Lambda}(f, X)}{\longrightarrow} \operatorname{Hom}_{\Lambda}(L, X)$ é exata.

c) A sequência $0 \rightarrow L_{\Lambda} \stackrel{f}{\rightarrow} M_{\Lambda} \stackrel{g}{\rightarrow} N_{\Lambda} \rightarrow 0$ é exata e cinde se, e somente se, para todo $X_{\Lambda}$, a sequência de k-módulos $0 \longrightarrow \operatorname{Hom}_{\Lambda}(X, L) \longrightarrow \operatorname{Hom}_{\Lambda}(X, M) \longrightarrow \operatorname{Hom}_{\Lambda}(X, N) \longrightarrow 0$ é exata.

d) A sequência $0 \rightarrow L_{\Lambda} \stackrel{f}{\rightarrow} M_{\Lambda} \stackrel{g}{\rightarrow} N_{\Lambda} \rightarrow 0$ é exata e cinde se, e somente se, para todo $X_{\Lambda}$, a sequência de k-módulos $0 \longrightarrow \operatorname{Hom}_{\Lambda}(N, X) \longrightarrow \operatorname{Hom}_{\Lambda}(M, X) \longrightarrow \operatorname{Hom}_{\Lambda}(L, X) \longrightarrow 0$ é exata.

\section{Módulos indecomponíveis, simples e projetivos}

Seja $\Lambda$ uma k-álgebra. Lembraremos nessa seção mais algumas definições e propriedades que serão utilizadas livremente no decorrer do texto.

Um $\Lambda$-módulo $M_{\Lambda}$ não nulo é dito indecomponível se sempre que $M=M_{1} \oplus M_{2}$, então $M_{1}=0$ ou $M_{2}=0$. Por outro lado, um $\Lambda$-módulo $S_{\Lambda}$ não nulo é dito simples se os seus únicos submódulos são os triviais, ou seja, $S_{\Lambda}$ e 0 . Um módulo que é soma direta de módulos simples, é dito semisimples. 
Definição 1.2 Um $\Lambda$-módulo $P_{\Lambda}$ é dito projetivo se satisfazer alguma das seguintes propriedades equivalentes:

1. para todo epimorfismo $f: M_{\Lambda} \rightarrow N_{\Lambda}$ e todo morfismo $g: P_{\Lambda} \rightarrow N_{\Lambda}$ existe um morfismo $\bar{g}: P_{\Lambda} \rightarrow M_{\Lambda}$ tal que $f \bar{g}=g$, ou seja, o seguinte diagrama é comutativo:

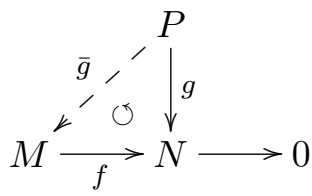

2. $P_{\Lambda}$ é um somando direto do $\Lambda$-módulo $\Lambda^{(T)}=\bigoplus_{t \in T} \Lambda_{t}$ onde $\Lambda_{t}=\Lambda$ para todo $t \in T$.

3. toda sequência exata curta da forma $0 \longrightarrow M \stackrel{f}{\longrightarrow} N \stackrel{g}{\longrightarrow} P \longrightarrow 0$ cinde.

4. o funtor $\operatorname{Hom}_{\Lambda}(P,-): \operatorname{Mod} \Lambda \rightarrow$ Mod k é exato, ou seja, dada uma sequência exata curta $0 \longrightarrow L \stackrel{f}{\longrightarrow} M \stackrel{g}{\longrightarrow} N \longrightarrow 0$ em Mod $\Lambda$, então a sequência induzida em Mod k, $0 \longrightarrow \operatorname{Hom}_{\Lambda}(P, L) \longrightarrow \operatorname{Hom}_{\Lambda}(P, M) \longrightarrow \operatorname{Hom}_{\Lambda}(P, N) \longrightarrow 0$ também é exata.

Definição 1.3 (Cobertura projetiva) Uma cobertura projetiva de um $\Lambda$-módulo $M$ é um par $(P, f)$, onde $P$ é um $\Lambda$-módulo projetivo e $f: P \rightarrow M$ é um epimorfismo supérfluo, isto é, $f$ é um epimorfismo com a seguinte propriedade:

- se $h: L \rightarrow P$ é morfismo tal que a composição $f h: L \rightarrow M$ é um epimorfismo, então $h$ é um epimorfismo.

Definição 1.4 Um submódulo $N$ de um $\Lambda$-módulo $M$ é dito supérfluo em $M$ se, para todo submódulo $L$ de $M$, tal que $N+L=M$ implicar que $L=M$.

Quando $\Lambda$ é uma álgebra de dimensão finita, a definição de $N$ ser supérfluo em $M$ é equivalente a dizer que $N \subseteq \operatorname{rad} M$.

Temos a seguinte (importante) caracterização de epimorfismos supérfluos.

Lema 1.5 (Nakayama) Sejam $\Lambda$ uma álgebra de dimensão finita e $M$ um $\Lambda$-módulo indecomponível. Então $f: M \rightarrow N$ é um epimorfismo supérfluo se, e somente se, Nuc $(f)$ é supérfluo em $M$.

Mostra-se, em [2] e [1] por exemplo, que todo módulo indecomponível sobre uma álgebra de dimensão finita admite cobertura projetiva. Além disso, tal cobertura projetiva é única no seguinte sentido:

- Se $(P, f)$ e $(\bar{P}, \bar{f})$ são coberturas projetivas de $M$, então existe um isomorfismo $h: \bar{P} \rightarrow P$ tal que $f h=\bar{f}$. 
Muitas vezes, a título de simplificação, diremos apenas que $P$ é uma cobertura projetiva de $M$, ou ainda, que $f: P \rightarrow M$ é uma cobertura projetiva de $M$.

Definição 1.5 (Apresentação projetiva) Uma apresentação projetiva de um $\Lambda$-módulo $M$ é uma sequência exata $P_{1} \stackrel{d_{1}}{\longrightarrow} P_{0} \stackrel{d_{0}}{\longrightarrow} M \rightarrow 0$ onde $P_{1}$ e $P_{0}$ são $\Lambda$-módulos projetivos. Tal apresentação é dita minimal se $d_{0}: P_{0} \rightarrow M$ e $d_{1}: P_{1} \rightarrow$ Nuc $\left(d_{0}\right)$ forem coberturas projetivas.

Passamos a definição do objeto de principal estudo neste texto.

Definição 1.6 (Resolução projetiva) Uma resolução projetiva de um $\Lambda$-módulo $M$ é uma sequência exata

$$
\cdots \rightarrow P_{n} \stackrel{d_{n}}{\longrightarrow} P_{n-1} \rightarrow \cdots \rightarrow P_{1} \stackrel{d_{1}}{\longrightarrow} P_{0} \stackrel{d_{0}}{\longrightarrow} M \rightarrow 0
$$

onde cada $P_{i}$ é um $\Lambda$-módulo projetivo.

Tal resolução é dita minimal se $d_{0}: P_{0} \rightarrow M$ e $d_{i}: P_{i} \rightarrow$ Nuc $\left(d_{i-1}\right), i \geq 1$, forem coberturas projetivas.

Uma resolução projetiva é dita de comprimento $n$ se $P_{n} \neq 0$ e $P_{i}=0, \forall i>n$. Neste caso, se $n$ for o menor inteiro com essa propriedade, dizemos que $M$ tem dimensão projetiva igual a $n e$ denotamos por dp $M$.

Chamamos de dimensão global de $\Lambda$, e denotamos por $\operatorname{dim} . g l . \Lambda, ~ o \sup \{\operatorname{dp} M: M \in \operatorname{Mod} \Lambda\}$. No caso em que $\Lambda$ é uma álgebra de dimensão finita, tem-se que

$$
\text { dim.gl. } \Lambda=\sup \{\operatorname{dp} S: S \text { é um } \Lambda \text {-módulo simples }\} .^{1}
$$

Teorema 1.6 (Teorema da Comparação) Sejam $M$ e $N$ dois $\Lambda$-módulos e $f: M \rightarrow N$ um morfismo de $\Lambda$-módulos. Se

$$
\cdots \rightarrow P_{n} \rightarrow P_{n-1} \rightarrow \cdots \rightarrow P_{1} \rightarrow P_{0} \rightarrow M \rightarrow 0
$$

é uma resolução projetiva para $M e$

$$
\cdots \rightarrow Q_{n} \rightarrow Q_{n-1} \rightarrow \cdots \rightarrow Q_{1} \rightarrow P_{0} \rightarrow N \rightarrow 0
$$

é uma sequência exata, então existe um diagrama comutativo da forma

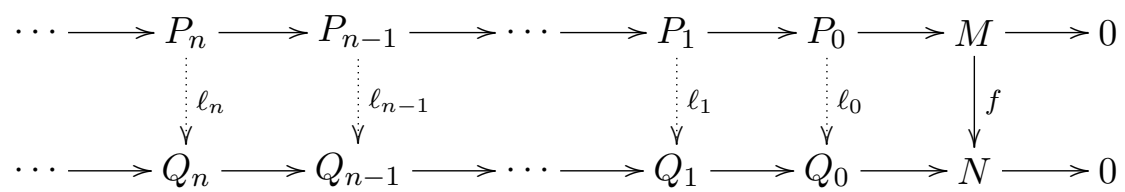

Os morfismos $\ell_{n}$ 's são chamados de "liftings" para $f$.

\footnotetext{
${ }^{1}$ Veja, por exemplo, [2].
} 
Proposição 1.7 Sejam $\Lambda$ uma k-álgebra de dimensão finita e $N$ um $\Lambda$-módulo semisimples. Se

$$
(*) \quad \cdots \rightarrow P_{n} \stackrel{d_{n}}{\longrightarrow} P_{n-1} \rightarrow \cdots \rightarrow P_{1} \stackrel{d_{1}}{\longrightarrow} P_{0} \stackrel{d_{0}}{\longrightarrow} M \rightarrow 0
$$

é uma resolução projetiva minimal para $M$, então $\operatorname{Hom}_{\Lambda}\left(P_{n}, N\right)=\operatorname{Nuc}\left(d_{n+1}^{*}\right)$ e $d_{n+1}^{*} \equiv 0$, para todo $n \geq 0$, onde $d_{i}^{*}:=\operatorname{Hom}_{\Lambda}\left(d_{i}, N\right)$.

\section{Prova.}

Para a primeira parte, mostraremos somente que $\operatorname{Hom}_{\Lambda}\left(P_{n}, N\right) \subseteq$ Nuc $\left(d_{n+1}^{*}\right)$, pois a outra é sempre verdadeira, não dependo do fato da resolução $(*)$ ser projetiva minimal.

Pela minimalidade da resolução $(*)$, temos que cada $n, \operatorname{Im}\left(d_{n+1}\right) \subseteq P_{n} \underline{r}$, onde $\underline{r}$ é o radical de Jacobson de $\Lambda$. Por outro lado, se $f \in \operatorname{Hom}_{\Lambda}\left(P_{n}, N\right)$ então $P_{n} \underline{\mathbf{r}} \subseteq \operatorname{Nuc}(f)$, pois $f\left(P_{n} \underline{r}\right) \subseteq N \underline{r}=$ $\operatorname{rad} N$ e dado que $N$ é semisimples $\operatorname{rad} N=0$. Assim $\operatorname{Im}\left(d_{n+1}\right) \subseteq P_{n} \underline{\underline{ }} \subseteq$ Nuc $(f)$ e, portanto, $d_{n+1}^{*}(f):=f d_{n+1}=0$. Isto mostra que $\operatorname{Hom}_{\Lambda}\left(P_{n}, N\right) \subseteq \operatorname{Nuc}\left(d_{n+1}^{*}\right)$ e que $d_{n+1}^{*} \equiv 0$.

\subsection{Produto tensorial de módulos}

Para essa seção, a menos de menção em contrário, k denotará um anel comutativo com unidade e $\Lambda$ uma k-álgebra.

Sejam $M_{\Lambda}$ e ${ }_{\Lambda} N \Lambda$-módulos e $W$ um k-módulo. Uma função $g: M \times N \rightarrow W$ é dita $\Lambda$-bilinear se para todos $m, m^{\prime} \in M, n, n^{\prime} \in N, x, x^{\prime} \in R$ e $a \in \Lambda$,

i) $g\left(m x+m^{\prime} x^{\prime}, n\right)=g(m, n) x+g\left(m^{\prime}, n\right) x^{\prime}$

ii) $g\left(m, n x+n^{\prime} x^{\prime}\right)=g(m, n) x+g\left(m, n^{\prime}\right) x^{\prime}$

iii) $g(m a, n)=g(m, a n)$.

Um produto tensorial de $M$ e $N$ é um par $(T, \tau)$, onde $T$ é um k-módulo e $\tau: M \times N \rightarrow T$ é uma aplicação $\Lambda$-bilinear, que tem a seguinte propriedade: para cada par $(W, g)$, onde $W$ é um k-módulo e $g: M \times N \rightarrow W$ é $\Lambda$-bilinear, existe uma única aplicação k-linear $\bar{g}: T \rightarrow W$ tal que $\bar{g} \tau=g$, ou seja, que o seguinte diagrama é comutativo:

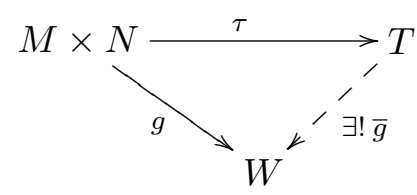

Dados os $\Lambda$-módulos $M_{\Lambda}$ e ${ }_{\Lambda} N$ existe, e é unico (a menos de isomorfismo), o produto tensorial de $M$ e $N$. Denotamos $T$ por $M \otimes_{\Lambda} N$, $\tau$ é chamada de função tensorial e $\tau(m, n)=m \otimes n$ são ditos tensores. Temos que $\tau(M \times N)$ gera $M \otimes_{\Lambda} N$, e então podemos escrever para cada $t \in M \otimes_{\Lambda} N$, $t=\sum_{i=1}^{n}\left(m_{i} \otimes n_{i}\right)$. Portanto, se $M$ e $N$ são finitamente gerados, então $M \otimes_{\Lambda} N$ também é finitamente gerado. 
Sejam $f: M_{\Lambda} \rightarrow M_{\Lambda}^{\prime}$ e $g: N_{\Lambda} \rightarrow N_{\Lambda}^{\prime}$ morfismos de $\Lambda$-módulos . A aplicação $f \times g: M \times N \rightarrow$ $M^{\prime} \times N$ definida por $(f \times g)(m, n):=(f(m), g(n))$ composta com a função tensorial de $M^{\prime} \otimes_{\Lambda} N^{\prime}$ é uma aplicação $\Lambda$-bilinear de $M \times N$ em $M^{\prime} \otimes_{\Lambda} N^{\prime}$. Logo induz uma aplicação k-linear denotada por $f \otimes g: M \otimes_{\Lambda} N \rightarrow M^{\prime} \otimes_{\Lambda} N^{\prime}$ que satisfaz $(f \otimes g)(m \otimes n)=f(m) \otimes g(n)$.

O próximo exemplo ilustra como são construídos isomorfismos que envolvem produtos tensoriais.

Exemplo 1.5 Seja $M_{\Lambda}$ um $\Lambda$-módulo. Então, $M \otimes_{\Lambda} \Lambda \cong M_{\Lambda}$. De fato, se definimos $f: M \times \Lambda \rightarrow$ $M$ por $f(m, a):=m a$, então é fácil verificar que $f$ é uma aplicação $\Lambda$-bilinear, e assim pela definição do produto tensorial, existe $\bar{f}: M \otimes_{\Lambda} \Lambda \rightarrow M$ k-linear tal que $\bar{f}(m \otimes a)=$ ma. Definimos também $g: M \rightarrow M \otimes_{\Lambda} \Lambda$ por $g(m):=m \otimes 1_{\Lambda}$. Temos que $g$ é k-linear e que $\bar{f} g=i d_{M}$ e $g \bar{f}=i d_{M \otimes_{\Lambda} N}$. Portanto, $M \otimes_{\Lambda} \Lambda \cong M_{\Lambda}$ como k-módulos. Porém, observamos que $\bar{f}$ e g são também morfismos de $\Lambda$-módulos, logo $M \otimes_{\Lambda} \Lambda \cong M_{\Lambda}$ também como $\Lambda$-módulos.

Mais ainda, o isomorfismo acima é funtorial, ou seja, $-\otimes_{\Lambda} \Lambda \approx i d_{\operatorname{Mod} \Lambda}$. Além disso, também é válido os seguintes isomorfismos funtoriais:

1. Se ${ }_{\Lambda} M$ é um módulo então $\Lambda \otimes_{\Lambda} M \cong_{\Lambda} M$.

2. Se $L_{\Lambda},{ }_{\Lambda} M_{\Sigma}$ e $N_{\Sigma}$ são três módulos, então $L \otimes_{\Lambda}\left(M \otimes_{\Sigma} N\right) \cong\left(L \otimes_{\Lambda} M\right) \otimes_{\Sigma} N$.

3. Se $I$ é um ideal bilateral de $\Lambda$ e $L_{\Lambda}$ um módulo qualquer, então $L \otimes_{\Lambda} \frac{\Lambda}{I} \approx \frac{L}{L I}$.

Proposição 1.8 (Teorema da adjunção) Sejam $\Lambda$ e $\Sigma$ k-álgebras $e_{\Sigma} M_{\Lambda}$ um ( $\Sigma$ - $\Lambda$ )-bimódulo. Os funtores $M \otimes_{\Lambda}-e \operatorname{Hom}_{\Sigma}(M,-)$ são adjuntos, ou seja, dados os módulos ${ }_{\Lambda} L$ e ${ }_{\Sigma} N$ existe o seguinte isomorfismo funtorial (em cada variável):

$$
\operatorname{Hom}_{\Lambda}\left(L, \operatorname{Hom}_{\Sigma}(M, N)\right) \approx \operatorname{Hom}_{\Sigma}\left(M \otimes_{\Lambda} L, N\right)
$$

Finalizaremos este tópico com um exemplo.

Exemplo 1.6 Sejam $\Lambda$ e $\Sigma$ duas k-álgebras. Então $\Lambda \otimes_{\mathrm{k}} \Sigma$ tem também uma estrutura natural de k-módulo. Além disso, $\Lambda \otimes_{\mathrm{k}} \Sigma$ tem também uma estrutura de k-álgebra dada pelo seguinte produto

$$
\left(a_{1} \otimes b_{1}\right) \cdot\left(a_{2} \otimes b_{2}\right):=\left(a_{1} a_{2}\right) \otimes\left(b_{1} b_{2}\right)
$$

com $a_{i} \in \Lambda$ e $b_{i} \in \Sigma$. No caso em que $\mathrm{k}$ é um corpo, a álgebra $\Lambda^{e}:=\Lambda^{o p} \otimes_{\mathrm{k}} \Lambda$ é chamada de álgebra envolvente de $\Lambda$. Vale salientar que se $M$ é um $\Lambda^{e}$-módulo (à direita), então podemos definir uma estrutura de ( $\Lambda$ - $\Lambda$ )-bimódulo para $M$ da seguinte forma

$$
a m:=m(a \otimes 1) \text { e } m b:=m(1 \otimes b)
$$


para todos $a, b \in \Lambda$ e $m \in M$. Por outro lado, se $M$ é um ( $\Lambda$ - $\Lambda$ )-bimódulo, então ele tem uma estrutura de $\Lambda^{e}$-módulo, dada por

$$
m(a \otimes b):=(a m) b=a(m b)
$$

para todos $a, b \in \Lambda$ e $m \in M$.

Talvez seja interessante lembrar que se $\Lambda$ é uma k-álgebra de dimensão finita, então existe uma equivalência entre a categoria de $\Lambda^{e}$-módulos à direita e a categoria de $(\Lambda$ - $\Lambda$ )-bimódulos.

\section{4 Álgebra de Caminhos}

Ao longo dessa seção vamos considerar k um corpo. Para um estudo mais amplo deste tópico, sugerimos ao leitor consultar $[1,2]$.

Um carcás $\Gamma$ é uma quádrupla $\left(\Gamma_{0}, \Gamma_{1}, o, t\right)$ onde $\Gamma_{0}$ e $\Gamma_{1}$ são conjuntos e o, $t: \Gamma_{1} \rightarrow \Gamma_{0}$ são funções. Os elementos de $\Gamma_{0}$ são chamados de vértices de $\Gamma$ e os elementos de $\Gamma_{1}$ são chamados de flechas de $\Gamma$. Dada uma flecha $a \in \Gamma_{1}$, chamamos o $(a)$ de origem de $a$ e t $(a)$ de término de a. Uma carcás $\Gamma$ é dito finito quando os conjuntos $\Gamma_{0}$ e $\Gamma_{1}$ são finitos.

Exemplo 1.7 Em geral, representamos um carcás por um diagrama da forma $\underset{i}{\stackrel{a}{\longrightarrow}} \dot{2} \stackrel{c}{\longrightarrow} \cdot \dot{3}$, por exemplo. Aqui temos $\Gamma_{0}=\{1,2,3\}, \Gamma_{1}=\{a, b, c\}, \mathrm{o}(a)=\mathrm{o}(b)=1, \mathrm{o}(c)=\mathrm{t}(a)=\mathrm{t}(b)=2 e$ $\mathrm{t}(c)=3$.

Um subcarcás de $\Gamma$ é um carcás $\left(\tilde{\Gamma}_{0}, \tilde{\Gamma}_{1}\right.$, õ, $\left.\tilde{\mathrm{t}}\right)$ de forma que $\tilde{\Gamma}_{0} \subseteq \Gamma_{0}, \tilde{\Gamma}_{1} \subseteq \Gamma_{1}$, o $=$ o $\left.\right|_{\tilde{\Gamma}_{1}}$ e $\tilde{\mathrm{t}}=\left.\mathrm{t}\right|_{\tilde{\Gamma}_{1}}$. Um subcarcás é dito pleno se a flecha $u \stackrel{a}{\rightarrow} v$ estiver em $\tilde{\Gamma}_{1}$ sempre que $u, v \in \tilde{\Gamma}_{0}$.

Um caminho $\omega$ em $\Gamma$ de comprimento $n>0$ é uma sequência de flechas $\omega=a_{1} \cdots a_{n}$, tal que $\mathrm{t}\left(a_{i}\right)=\mathrm{o}\left(a_{i+1}\right)$ para $1 \leq i<n$. Denotamos o comprimento de $\omega$ por $\ell(\omega)$. Dizemos que $\gamma=a_{i} \cdots a_{i+t}$ é um subcaminho de $\omega$ se $1 \leq i, i+t \leq n$. Por convenção, um caminho de comprimento zero (ou caminho trivial) é um caminho sem flechas associado a um vértice $v \in \Gamma_{0}$, que denotamos por $e_{v}$. Para um caminho não trivial $\omega=a_{1} \cdots a_{n}$ definimos o vértice inicial (ou origem) de $\omega$ por $\mathrm{o}(\omega):=\mathrm{o}\left(a_{1}\right)$ e o vértice final (ou término) de $\omega \operatorname{por} \mathrm{t}(\omega):=\mathrm{t}\left(a_{n}\right)$. Para um caminho trivial $e_{v}$ definimos o $\left(e_{v}\right)=\mathrm{t}\left(e_{v}\right)=v$. (Na maior parte do texto, denotaremos este "caminho trivial" apenas pelo vértice que o representa.) Um caminho $\omega$ de comprimento $n \geq 1$ é dito um ciclo orientado quando o $(\omega)=\mathrm{t}(\omega)$.

Consideremos agora $\Gamma$ um carcás finito. Seja $k \Gamma$ o k-espaço vetorial cuja base é o conjunto de todos os caminhos de $\Gamma$. Definimos em $\mathrm{k} \Gamma$ o seguinte produto: dados $\gamma$ e $\sigma$ caminhos de $\Gamma$, então

- $\operatorname{set}(\gamma) \neq \mathrm{o}(\sigma), \gamma \cdot \sigma=0$

- $\operatorname{set}(\gamma)=\mathrm{o}(\sigma), \gamma \cdot \sigma=\left\{\begin{array}{cll}\sigma, & \text { se } & \gamma=e_{v} \text { para algum } v \in \Gamma_{0} \\ \gamma, & \text { se } & \sigma=e_{v} \text { para algum } v \in \Gamma_{0} \\ a_{1} \cdots a_{n} b_{1} \cdots b_{t}, & \text { se } & \gamma=a_{1} \cdots a_{n} \text { e } \sigma=b_{1} \cdots b_{t}\end{array}\right.$ 
Estendendo esse produto, por linearidade, aos elementos de $k \Gamma$ temos que $k \Gamma$ é uma k-álgebra, a qual chamamos de álgebra de caminhos de $\Gamma$.

Exemplo 1.8 Seja Гo carcás

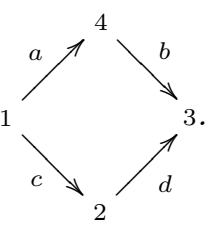

$A$ base de $\mathrm{k} \Gamma$ como $\mathrm{k}$-espaço vetorial é $\left\{e_{1}, e_{2}, e_{3}, e_{4}, a, b, c, d, a b, c d\right\}$ e portanto a dimensão de $\mathrm{k} \Gamma$ é $\operatorname{dim}_{\mathrm{k}} \mathrm{k} \Gamma=10$. Quanto a multiplicação teremos, por exemplo, $a \cdot b=a b, a \cdot d=0, e_{2} \cdot d=d$, etc. $\triangleleft$

Definição 1.7 Um elemento $x$ em $\mathrm{k} \Gamma$ é chamado uniforme à direita (à esquerda) se existe um vértice $v \in \Gamma_{0}$ tal que $x v=x(v x=x)$. Se ele for uniforme à direita e à esquerda, ele é dito uniforme.

Uma relação $\rho$ em $\Gamma$ é uma combinação linear de caminhos uniformes, onde cada caminho tem comprimento pelo menos dois.

Denotaremos por $J_{\Gamma}$, ou simplesmente $J$, o ideal de $\mathrm{k} \Gamma$ gerado pelas flechas de $\Gamma$. Na maior parte do texto, nossas álgebras serão quocientes, $\mathrm{k} \Gamma / I$, de álgebras de caminhos onde o ideal $I$ de $\mathrm{k} \Gamma$ está contido em $J^{2}$.

Se $I$ é um ideal de $\mathrm{k} \Gamma$ gerado por combinações lineares de caminhos de comprimento dois, dizemos que $\Lambda=\mathrm{k} \Gamma / I$ é uma álgebra quadrática. Por outro lado, se cada gerador de $I$ for um caminho de $\Gamma$, dizemos que $\Lambda$ é uma álgebra monomial.

Proposição 1.9 Sejam $\Gamma$ um carcás finito com $\Gamma_{0}=\{1, \ldots, n\}$, $\mathrm{k} \Gamma$ sua álgebra de caminhos e $e_{i} o$ caminho trivial associado ao vértice $i \in \Gamma_{0}$. Então:

1. k $\Gamma$ é uma álgebra associativa.

2. o conjunto $\left\{e_{i}\right\}_{i \in \Gamma_{0}}$ é um sistema completo de idempotentes ortogonais primitivos de $\mathrm{k} \Gamma$. Em particular, $\mathrm{k} \Gamma$ tem identidade $1=e_{1}+\cdots+e_{n}$.

3. $\mathrm{k} \Gamma$ é uma álgebra básica, isto é, $\mathrm{k} \Gamma=e_{1}(\mathrm{k} \Gamma) \oplus \cdots \oplus e_{n}(\mathrm{k} \Gamma)$ é a decomposição de $\mathrm{k} \Gamma$ em módulos projetivos indecomponíveis, dois a dois não isomorfos.

4. $\mathrm{k} \Gamma$ tem dimensão finita se, e somente se, $\Gamma$ não possui ciclos orientados.

5. $J_{\Gamma}=\underline{\mathrm{r}}$ se, e somente se, $\Gamma$ não possui ciclos orientados.

6. o número de flechas de $i$ para $j$ é igual ao número $\operatorname{dim}_{\mathrm{k}}\left(e_{i}\left(\frac{J_{\Gamma}}{J_{\Gamma}^{2}}\right) e_{j}\right)$.

Teorema 1.10 Seja $\Lambda$ uma k-álgebra básica e de dimensão finita sobre um corpo algebricamente fechado $\mathrm{k}$. Existe um carcás $\Gamma_{\Lambda}$ e um epimorfismo $\eta_{\Lambda}: \mathrm{k} \Gamma_{\Lambda} \rightarrow \Lambda$ onde $I_{\Lambda}:=$ Nuc $\eta_{\Lambda}$ é um ideal de $\mathrm{k} \Gamma_{\Lambda}$ tal que $J^{n} \subseteq I_{\Lambda} \subseteq J^{2}$, para algum $n \geq 2$.

O epimorfismo do teorema acima é chamado de uma apresentação de $\Lambda$ e o carcás $\Gamma_{\Lambda}$ é chamado de carcás ordinário de $\Lambda$. 


\section{Módulos e representações de carcáses}

Sejam k um corpo e $\Gamma$ um carcás finito. Uma representação de $\Gamma$ é dada por $V=\left(\left(V_{i}\right)_{i \in \Gamma_{0}},\left(T_{a}\right)_{a \in \Gamma_{1}}\right)$, onde para cada $i \in \Gamma_{0}, V_{i}$ é um k-espaço vetorial de dimensão finita e para cada $a \in \Gamma_{1}, T_{a}$ é uma transformação linear de $V_{\mathrm{o}(a)}$ em $V_{\mathrm{t}(a)}$.

Exemplo 1.9 Seja $\Gamma$ o carcás $1 \stackrel{a}{\longleftarrow} 2$. Então $\mathrm{k}^{2} \underset{T_{b}}{\stackrel{T_{a}}{\longleftarrow}} \mathrm{k}$ é uma representação de $\Gamma$, onde $V_{1}=\mathrm{k}^{2}, V_{2}=\mathrm{k}, T_{a}=\left[\begin{array}{l}1 \\ 0\end{array}\right]$ e $T_{b}=\left[\begin{array}{l}0 \\ 1\end{array}\right]$. Observe que $\mathrm{k}^{2} \underset{T_{b}^{\prime}}{\stackrel{T_{a}^{\prime}}{\longleftarrow}} \mathrm{k}^{2}$ também é uma representação de $\Gamma$, onde $V_{1}=\mathrm{k}^{2}, V_{2}=\mathrm{k}^{2}, T_{a}^{\prime}=\left[\begin{array}{ll}1 & 0 \\ 0 & 1\end{array}\right]$ e $T_{b}^{\prime}=\left[\begin{array}{ll}0 & 0 \\ 1 & 0\end{array}\right]$.

Seja $\omega=a_{1} a_{2} \ldots a_{n}$ um caminho não trivial de $\Gamma$. Definimos a transformação linear $T(\omega): V_{\mathrm{o}(\omega)} \rightarrow V_{\mathrm{t}(\omega)}$ dada pela composta $T_{a_{1}} T_{a_{2}} \cdots T_{a_{n}}$. Estendemos esta definição para uma combinação linear de caminhos $\omega=\sum_{i=1}^{t} \lambda_{i} \omega_{i}$, onde o $\left(\omega_{i}\right)=\mathrm{o}\left(\omega_{j}\right) \operatorname{et}\left(\omega_{i}\right)=\mathrm{t}\left(\omega_{j}\right), \forall i, j \in\{1, \ldots, n\}$, fazendo $T(\omega)=\sum_{i=1}^{t} \lambda_{i} T\left(\omega_{i}\right)$.

Uma representação de $(\Gamma, I)$ é uma representação de $\Gamma$ de forma que para cada relação $\omega$ de $I$ tem-se $T(\omega)=0$.

Dadas duas representações $V=\left(\left(V_{i}\right)_{i \in \Gamma_{0}},\left(T_{a}\right)_{a \in \Gamma_{1}}\right)$ e $W=\left(\left(W_{i}\right)_{i \in \Gamma_{0}},\left(S_{a}\right)_{a \in \Gamma_{1}}\right)$, um morfismo $\Phi: V \rightarrow W$ é uma família $\left\{\phi_{i}\right\}_{i \in \Gamma_{0}}$ de transformações lineares tal que, para cada flecha $i \stackrel{a}{\rightarrow} j \mathrm{o}$ seguinte diagrama é comutativo

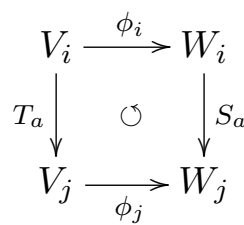

ou seja, $\phi_{j} T_{a}=S_{a} \phi_{i}$. A composta de dois morfismos é definida coordenada a coordenada. Definimos então a categoria mod $(\Gamma, I)$ cujos objetos são as representações de $(\Gamma, I)$ e os morfismos são os descritos acima.

Exemplo 1.10 Continuando o exemplo acima, observe que

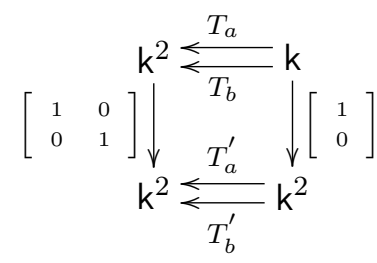

é um morfismo entre as duas representações.

Teorema 1.11 Seja $\Lambda=\mathrm{k} \Gamma / I$ onde $\Gamma$ é um carcás finito e $I$ um ideal de $\mathrm{k} \Gamma$ tal que $J^{n} \subseteq I \subseteq J^{2}$, para algum $n \geq 2$. Então as categorias $\bmod (\Gamma, I)$ e $\bmod \Lambda$ são equivalentes. 
Pelo teorema acima podemos identificar os $\Lambda$-módulos com representações de $(\Gamma, I)$. Em especial, as representações que correspondem aos $\Lambda$-módulos simples, projetivos indecomponíveis e injetivos indecomponíveis podem ser calculados a partir do carcás ordinário $(\Gamma)$ e das relações $(I)$. Para detalhes dessas descrições ver, por exemplo, [1,2].

\section{5 Álgebras Graduadas}

Assim como na seção anterior, $\mathrm{k}$ denotará um corpo.

Nesta seção, abordaremos alguns conceitos da teoria de módulos graduados sobre uma álgebra graduada. Para isso, seguimos basicamente [18,22].

Uma k-álgebra $\Lambda$ é dita (positivamente) graduada quando $\Lambda=\bigoplus_{n \geq 0} \Lambda_{n}$, onde os $\Lambda_{n}$ 's são k-espaços vetoriais tais que $\Lambda_{i} \Lambda_{j} \subseteq \Lambda_{i+j}$, para todos $i, j \geq 0$. Os elementos não nulos de $\Lambda_{j}$ são chamados de elementos homogêneos de grau $j$. Dizemos que uma k-álgebra graduada $\Lambda$ é gerada em graus 0 e 1 quando $\Lambda_{i} \Lambda_{j}=\Lambda_{i+j}$, para todos $i, j \geq 0$ ou, equivalentemente, quando $\Lambda_{k}=\prod_{i=1}^{k} \Lambda_{1}$, para todo $k \geq 1$. De fato, se $\Lambda_{i} \Lambda_{j}=\Lambda_{i+j}$ e $\Lambda_{n}=\prod_{i=1}^{n} \Lambda_{1}$, para todo $n \leq k$, então $\Lambda_{k+1}=\Lambda_{k} \Lambda_{1}=\left(\prod_{i=1}^{k} \Lambda_{1}\right) \Lambda_{1}=\prod_{i=1}^{k+1} \Lambda_{1}$. Reciprocamente, $\Lambda_{i} \Lambda_{j}=\left(\prod_{l=1}^{j} \Lambda_{1}\right)\left(\prod_{l=1}^{i} \Lambda_{1}\right)=$ $\prod_{l=1}^{i+j} \Lambda_{1}=\Lambda_{i+j}$

Um ideal $I$ de uma álgebra graduada $\Lambda$ é dito graduado ${ }^{2}$ quando $I=\bigoplus_{n \geq 0} I_{n}$, onde cada componente $I_{j}$ é um k-espaço vetorial e para cada $k$ e $j$ tem-se que $I_{j} \Lambda_{k} \subseteq I_{j+k}$. Observe que isto é equivalente a dizer que cada elemento de $I=\bigoplus_{n \geq 0} I_{n}$ é uma soma de elementos homogêneos.

Exemplo 1.11 (a) Seja $\mathrm{k} \Gamma$ a álgebra de caminhos de um carcás $\Gamma$ sobre k. Para cada $n \geq 0$, denotamos por $(\mathrm{k} \Gamma)_{n}$ o $\mathrm{k}$-espaço vetorial gerado pelos caminhos de comprimento $n$ em $\Gamma$. Então, como $\mathrm{k}$-espaço vetorial temos $\mathrm{k} \Gamma=\bigoplus_{n \geq 0}(\mathrm{k} \Gamma)_{n}$. Além disso, dado que o comprimento do produto de caminhos é igual a soma dos comprimentos (no caso não nulo) de cada caminho segue que $(\mathrm{k} \Gamma)_{m}(\mathrm{k} \Gamma)_{n} \subseteq(\mathrm{k} \Gamma)_{m+n}$, para todos $m, n \geq 0$, e com isso $\mathrm{k} \Gamma$ é uma álgebra graduada. Por outro lado, como todo caminho de comprimento $n \geq 1$ é produto de $n$ flechas, então $(\mathrm{k} \Gamma)_{n}=\prod_{i=1}^{n}(\mathrm{k} \Gamma)_{1}$, o que mostra que $\mathrm{k} \Gamma$ é uma álgebra graduada gerada em graus 0 e 1.

(b) Sejam $\Lambda=\bigoplus_{n \geq 0} \Lambda_{n}$ e $\Sigma=\bigoplus_{n \geq 0} \Sigma_{n}$ álgebras graduadas. Então o produto tensorial $\Lambda \otimes_{\mathrm{k}} \Sigma$ é uma álgebra graduada com graduação dada por

$$
\Lambda \otimes_{\mathrm{k}} \Sigma=\bigoplus_{n \geq 0}\left(\sum_{i+j=n} \Lambda_{i} \otimes_{\mathrm{k}} \Sigma_{j}\right) .
$$

Além disso, se $\Lambda$ e $\Sigma$ são geradas em graus 0 e 1 , então $\Lambda \otimes_{\mathrm{k}} \Sigma$ também é gerada em graus 0 e 1 . Por exemplo,

$$
\begin{aligned}
\left(\Lambda \otimes_{\mathrm{k}} \Sigma\right)_{1}\left(\Lambda \otimes_{\mathrm{k}} \Sigma\right)_{1} & =\left(\Lambda_{0} \otimes_{\mathrm{k}} \Sigma_{1}+\Lambda_{1} \otimes_{\mathrm{k}} \Sigma_{0}\right)\left(\Lambda_{0} \otimes_{\mathrm{k}} \Sigma_{1}+\Lambda_{1} \otimes_{\mathrm{k}} \Sigma_{0}\right) \\
& =\Lambda_{0} \otimes_{\mathrm{k}} \Sigma_{2}+\Lambda_{1} \otimes_{\mathrm{k}} \Sigma_{1}+\Lambda_{1} \otimes_{\mathrm{k}} \Sigma_{1}+\Lambda_{2} \otimes_{\mathrm{k}} \Sigma_{0} \\
& =\Lambda_{0} \otimes_{\mathrm{k}} \Sigma_{2}+\Lambda_{1} \otimes_{\mathrm{k}} \Sigma_{1}+\Lambda_{2} \otimes_{\mathrm{k}} \Sigma_{0} \\
& =\left(\Lambda \otimes_{\mathrm{k}} \Sigma\right)_{2} .
\end{aligned}
$$

\footnotetext{
${ }^{2}$ Alguns autores também chamam ideal graduado de ideal homogêneo.
} 
(c) Dada uma álgebra $\Lambda$ e um ideal $I$ de $\Lambda$, considere o anel $\operatorname{Gr}_{I}(A)=\bigoplus_{n \geq 0} I^{n} / I^{n+1}$, com o produto dado por

$$
\left(x+I^{n+1}\right) \cdot\left(y+I^{m+1}\right)=\left(x y+I^{n+m+1}\right), \text { para todos } m, n \geq 0, \text { com } I^{0}:=A .
$$

Então $\operatorname{Gr}_{I}(A)$ é um anel graduado gerado em graus 0 e 1 .

A proposição que se segue nos dá uma espécie de recíproca para o item (c) do exemplo acima.

Proposição 1.12 ([18]) Sejam $\Lambda=\bigoplus_{n \geq 0} \Lambda_{n}$ um anel graduado gerado em grau 0 e 1 , e $J=$ $\bigoplus_{n \geq 1} \Lambda_{n}$. Então $J$ é um ideal de $\Lambda$ tal que $\Lambda$ é isomorfo (como anéis graduados) $a \bigoplus_{n \geq 0} J^{n} / J^{n+1}$, onde $J^{0}:=\Lambda$

Prova.

Dado que $\Lambda_{i \geq 0} \Lambda_{j \geq 1} \subseteq \Lambda_{i+j \geq 1}$ e que $\Lambda_{i \geq 1} \Lambda_{j \geq 0} \subseteq \Lambda_{i+j \geq 1}$, então $J \Lambda \subset J$ e $\Lambda J \subset J$. (Observe que para isso não usamos o fato de $\Lambda$ ser gerado em graus 0 e 1.) Além disso, como $J^{0}:=\Lambda$, então $J^{0} / J$ é isomorfo a $\Lambda_{0}$. Agora como $\Lambda$ é gerado em grau 0 e $1(\star)$, temos que

$$
J^{2}=\sum_{n \geq 2}\left(\sum_{i+j=n} \Lambda_{i} \Lambda_{j}\right) \stackrel{\star}{=} \sum_{n \geq 2} \Lambda_{n}=\bigoplus_{n \geq 2} \Lambda_{n} .
$$

Logo, $J / J^{2}$ é isomorfo a $\Lambda_{1}$. Seguindo esse racicínio, teremos que $J^{k}=\bigoplus_{n \geq k} \Lambda_{n}$ e, portanto, $J^{k} / J^{k+1} \simeq \Lambda_{k}$.

O ideal $J$ definido na proposição acima é um ideal "especial", pois se $I=\bigoplus_{n \geq 0} I_{n}$ é um ideal graduado à esquerda do anel graduado $\Lambda=\bigoplus_{n \geq 0} \Lambda_{n}$ tal que $J+I=\Lambda$, então $I=\Lambda$.

De fato, como $I_{0}:=I \cap \Lambda_{0}$, então $I_{0} \subseteq \Lambda_{0}$. Por outro lado, se $x_{0} \in \Lambda_{0}$, então $x_{0}=$ $\dot{\sum}_{s \geq 0} i_{s}+\dot{\sum}_{t \geq 1} \lambda_{t}$, com cada $i_{s} \in I_{s}$ e cada $\lambda_{t} \in \Lambda_{t}$. Assim $x_{0}-i_{0}=\dot{\sum}_{s \geq 1} i_{s}+\dot{\sum}_{t \geq 1} \lambda_{t} \in \underline{r}$, o que implica que $x_{0}=i_{0} \in I_{0}$. Isto mostra que $I_{0}=\Lambda_{0}$. Além disso, dados $\lambda_{s} \in \Lambda_{s} \mathrm{e}$ $i_{0} \in I_{0} \subseteq I$, tem-se que $\lambda_{s} i_{0} \in I \cap \Lambda_{s}:=I_{s}$ (pois $\Lambda_{s} I_{0}=\Lambda_{s} \Lambda_{0} \subseteq \Lambda_{s}$ ), ou seja, $\Lambda_{s} I_{0} \subseteq I_{s}$. Mas, $1 \in \Lambda_{0}=I_{0}$ implica que $I_{s}=\Lambda_{s}$ para todo $s \geq 0$ e com isso concluímos que $I=\Lambda$.

Acabamos de mostrar que o ideal $J$ é um ideal supérfluo, justificando assim a seguinte definição:

Definição 1.8 Seja $\Lambda=\bigoplus_{n \geq 0} \Lambda_{n}$ um anel graduado tal que $\Lambda_{0}$ é um anel semi-simples, então dizemos que o ideal $\underline{\mathbf{r}}=\bigoplus_{n \geq 1} \Lambda_{n}$ é o radical de Jacobson graduado de $\Lambda$.

Observação 1.1 O radical de Jacobson graduado é a interseção dos ideais maximais graduados de $\Lambda$. Como consequência anula todos os módulos graduados simples.

Desta observação, obtemos uma versão graduada da Proposição 1.7. 
Se $\Lambda$ é uma k-álgebra graduada, então um $\Lambda$-módulo $M$ é dito graduado quando $M=$ $\bigoplus_{n \geq 0} M_{n}$, onde para cada $k$ e $j$ tem-se que $M_{j} \Lambda_{k} \subseteq M_{k+j}$ e cada componente $M_{i}$ é um k-espaço vetorial. Dado que $M_{n} \Lambda_{0} \subseteq M_{n}$, então cada $M_{n}$ é um $\Lambda_{0}$-módulo. Um $\Lambda$-módulo graduado $M=\bigoplus_{n \geq 0} M_{n}$ é gerado em grau $k$ se $M_{n}=0$, para todo $n<k$, e $M_{n}=M_{k} \Lambda_{n-k}$, sempre que $n \geq k$. Os elementos não nulos de $M_{n}$ são chamados de elementos homogênios de grau $n$. Observe que um ideal de $\Lambda$ é graduado se é um $\Lambda$-módulo graduado.

Dados dois $\Lambda$-módulos graduados $M$ e $N$, um $\Lambda$-homomorfismo $f: M \rightarrow N$ é dito de grau $j$ se $f\left(M_{n}\right) \subseteq N_{n+j}$, para todo $n \geq 0$. Em particular, é dito de grau 0 se $f\left(M_{n}\right) \subseteq N_{n}$, para todo $n \geq 0$.

Para uma dada k-álgebra graduda $\Lambda$ denotaremos por $\operatorname{Gr} \Lambda$ a categoria que tem como objetos os $\Lambda$-módulos (à direita) graduados e como morfismos as aplicações de grau 0 , isto é, dados $M=$ $\bigoplus_{n \geq 0} M_{n}$ e $N=\bigoplus_{n \geq 0} N_{n}$ dois $\Lambda$-módulos graduados, então

$$
\operatorname{Hom}_{\mathrm{Gr} \Lambda}(M, N):=\left\{f \in \operatorname{Hom}_{\Lambda}(M, N): f\left(M_{k}\right) \subseteq N_{k}, \text { para todo } k \geq 0\right\}
$$

A categoria $\operatorname{Gr} \Lambda$ possui somas diretas e produtos. De fato, para um família de $\Lambda$-módulos graduados $\left\{M_{\sigma}\right\}_{\sigma \in \Sigma}$, uma soma direta $S_{\Sigma}=\bigoplus_{n \geq 0} S_{n}$ pode ser dada fazendo $S_{n}=\bigoplus_{\sigma}\left(M_{\sigma}\right)_{n}$ e um produto pode ser obtido tomando $P_{n}=\prod_{\sigma}\left(M_{\sigma}\right)_{n}$ e então $P_{\Sigma}=\bigoplus_{n \geq 0} P_{n}$.

Exemplo 1.12 Sejam $M$ e $N$ dois $\Lambda$-módulos graduados. Então o $\Lambda$-módulo graduado $M \oplus N$ é gerado em grau $j$ se, e somente se, $M$ e $N$ são ambos gerados em grau $j$.

Exemplo 1.13 Sejam $\Lambda$ e $\Sigma$ álgebras graduadas e $M_{\Lambda}$ e $N_{\Sigma}$ dois módulos graduados. Então $M \otimes_{\mathrm{k}} N$ é um módulo graduado sobre $\Lambda \otimes_{\mathrm{k}} \Sigma$, com graduação dada por

$$
M \otimes_{\mathrm{k}} N=\bigoplus_{n \geq 0}\left(\sum_{i+j=n} M_{i} \otimes_{\mathrm{k}} N_{j}\right) .
$$

Além disso, se $M_{j}$ é gerado em grau $i$ e $N$ em grau $j$, então $M \otimes_{\mathrm{k}} N$ é gerado em grau $i+j$.

A categoria dos $\Lambda$-módulos graduados finitamente gerados será denotada por gr $\Lambda$. Mais precisamente, gr $\Lambda$ é a subcategoria plena de $\operatorname{Gr} \Lambda$ que tem como objetos aqueles objetos $M$ de $\operatorname{Gr} \Lambda$ tais que $F(M) \in \bmod \Lambda$, onde $F: \operatorname{Gr} \Lambda \rightarrow \operatorname{Mod} \Lambda$ é o funtor esquecimento, ou seja, o funtor que a cada módulo graduado $M$ associa o módulo $M$ (desconsiderando a graduação de $M$ ) e cada morfismo $f$ associa o morfismo $f$.

Dado $M \in \operatorname{Gr} \Lambda$ e $d$ um inteiro qualquer, definimos $d$-shift de $M$ como sendo o $\Lambda$-módulo graduado $M(d)=\bigoplus_{m \geq 0} M(d)_{m}$, onde $M(d)_{m}:=M_{m+d}$. Isto define um funtor $T_{d}: \operatorname{Gr} \Lambda \ni M \longmapsto$ $M(d) \in \operatorname{Gr} \Lambda$. Observe que para quaisquer que sejam os inteiros $b$ e $d$, temos que $T_{d} \circ T_{b}=T_{d+b}$ e, portanto, $T_{d} \circ T_{-d}=\mathrm{Id}$. Em particular, $T_{d}$ é um isomorfismo na categoria $\operatorname{Gr} \Lambda$, para todo $d \in \mathbb{Z}$. Observe também que $F \circ T_{d}=F$. 
Em parte deste trabalho, restrigiremos nossa atenção a uma classe especial de álgebras graduadas, que serão as álgebras graduadas $\Lambda=\bigoplus_{n \geq 0} \Lambda_{n}$ tais que:

- $\Lambda$ é gerada em graus 0 e 1

- $\operatorname{dim}_{\mathrm{k}} \Lambda_{1}<\infty$

- $\Lambda_{0}$ é isomorfo a um produto finito de cópias do corpo $\mathrm{k}$, ou seja, $\Lambda_{0} \simeq \mathrm{k} \times \cdots \times \mathrm{k}$.

Uma álgebra graduada dessa forma será chamada de 0-1-split-básica. A partir de agora, a menos de menção em contrário, assumiremos que as álgebras graduadas são 0,1-split-básicas, omitindo assim este termo.

Exemplo 1.14 (a) Sejam $\Gamma$ um carcás finito e $R=\mathrm{k} \Gamma$ a álgebra de caminhos de $\Gamma$ sobre um corpo k. Como sabemos $R=\bigoplus_{n \geq 0}(\mathrm{k} \Gamma)_{n}$ é uma graduação para $R$, onde $(\mathrm{k} \Gamma)_{n}$ é o $\mathrm{k}$-espaço vetorial gerado pelos caminhos em $\Gamma$ de comprimento $n$. Além disso $R$ é gerada em graus 0 e 1 e como $\Gamma$ é finito, segue que $\operatorname{dim}_{\mathrm{k}} \Lambda_{1}<\infty$ e que $(\mathrm{k} \Gamma)_{0}$ é isomorfo a um produto finito de cópias de $\mathrm{k}$. Portanto $R$ é uma álgebra graduada 0,1-split-básica.

(b) Se $R$ é como acima e I é um ideal graduado de $R$ tal que $I \subseteq J^{2}$, onde $J=\bigoplus_{n \geq 1}(\mathrm{k} \Gamma)_{n}$, então $R / I$ é uma álgebra graduada 0,1-split-básica com radical graduado $\underline{\mathrm{r}}=\mathrm{J} / \mathrm{I}^{3}$

O próximo resultado nos mostra uma caracteriazação das álgebras graduadas 0,1-split-básicas. (Compare com o exemplo acima!)

Proposição 1.13 ([18]) Seja $\Lambda=\bigoplus_{n \geq 0} \Lambda_{n}$ uma k-álgebra graduada 0,1-split-básica. Então existem um carcás finito $\Gamma$ e um ideal graduado I em $\mathrm{k} \Gamma$, contido em $J^{2}$, tais que $\Lambda$ é isomorda a $\mathrm{k} \Gamma / I$ como k-álgebras graduadas.

Talvez seja interessante destacar que na prova da proposição acima, o carcás $\Gamma$ e o ideal $I$ são obtidos da seguinte forma:

- o conjunto dos vértices $\left\{v_{1}, \ldots, v_{n}\right\}$ está em correspondência biunívoca com o conjunto $\left\{e_{1}, \ldots, e_{n}\right\}$ de idempotentes ortogonais primitivos centrais de $\Lambda_{0}$;

- o número de flechas de $v_{i}$ para $v_{j}$ é igual a $n_{i, j}=\operatorname{dim}_{\mathrm{k}}\left(e_{j}\left(\underline{\mathrm{r}} / \underline{\mathrm{r}}^{2}\right) e_{i}\right)$, onde $\underline{\mathrm{r}}=\bigoplus_{n \geq 1} \Lambda_{n}$. Observe que cada $n_{i, j}<\infty$, pois pela Proposição $1.12 \underline{\mathrm{r}} / \underline{\mathrm{r}}^{2} \cong \Lambda_{1}$ e por hipótese $\operatorname{dim}_{\mathrm{k}} \Lambda_{1}<\infty$.

- o ideal $I$ é o núcleo do epimorfismo $\psi: \mathrm{k} \Gamma \rightarrow \Lambda$ dado por $\psi\left(v_{i}\right)=e_{i}$ e $\psi\left(\alpha_{i, j}^{s}\right)=\beta_{i, j}^{s}$, onde para cada $1 \leq i, j \leq n,\left\{\beta_{i, j}^{s}\right\}_{s=1}^{n_{i, j}}$ é uma k-base fixada para o k-espaço vetorial $e_{j}\left(\underline{\mathrm{r}} / \underline{\mathrm{r}}^{2}\right) e_{i}$ e $\left\{\alpha_{i, j}^{s}\right\}_{s}$ denota o conjunto das $n_{i, j}$ flechas de $v_{i}$ para $v_{j}$.

\footnotetext{
${ }^{3}$ Observe que não estamos assumindo que $R / I$ tem dimensão finita.
} 
Usando a notação acima temos que $e_{i} \Lambda$ é um $\Lambda$-módulo projetivo graduado, com $\left(e_{i} \Lambda\right)_{n}:=e_{i} \Lambda_{n}$. Neste caso, assumiremos que cada um desses projetivos está gerado em grau 0. Além disso, quando falarmos de um $\Lambda$-módulo projetivo graduado (finitamente gerado) estaremos supondo que este é uma soma direta (finita) da forma $\bigoplus_{j} e_{i_{j}} \Lambda\left(s_{j}\right)\left(\bigoplus_{j=1}^{m} e_{i_{j}} \Lambda\left(s_{j}\right)\right)$, isto é, uma soma direta de cópias $\operatorname{dos} e_{i} \Lambda$ 's "shiftiados". Os $\Lambda$-módulos simples graduados são isomomorfos a algum $S_{i}=e_{i}(\Lambda / J)$, onde, como um $\Lambda$-módulo graduado, $\left(S_{i}\right)_{0}=e_{i}\left(\Lambda_{0}\right)$ e $\left(S_{i}\right)_{j}=0$ se $j \neq 0$.

Sejam $M, P \in \operatorname{gr} \Lambda, p: P \rightarrow M$ um epimorfirmo de grau 0 e $P$ um $\Lambda$-módulo projetivo tais que Nuc $(p) \subseteq \operatorname{rad} P:=P \underline{r}$. Então o par $(p, P)$ é chamado de cobertura projetiva graduada de $M$. É sabido ${ }^{4}$ que todo módulo $M \in \operatorname{gr} \Lambda$ possui cobertura projetiva $p: P \rightarrow M$ e que $P$ é gerado em grau $j$ sempre $M$ for gerado em grau $j$. Além disso, se $g: Q \rightarrow M$ é uma outra cobertura projetiva graduada para $M$, então existe um isomorfismo de módulos graduados $h: P \rightarrow Q$ tal que $g h=p$.

Definição 1.9 Seja $M$ um $\Lambda$-módulo graduado. Por uma resolução projetiva graduada para $M$, entendemos uma sequência exata

$$
\cdots \rightarrow P_{n} \stackrel{d_{n}}{\longrightarrow} P_{n-1} \rightarrow \cdots \rightarrow P_{1} \stackrel{d_{1}}{\longrightarrow} P_{0} \stackrel{d_{0}}{\longrightarrow} M \rightarrow 0
$$

onde, para cada $i \geq 0, P_{i}$ é um $\Lambda$-módulo projetivo graduado e cada aplicação $d_{i}$ é um $\Lambda$ homomorfismo de grau 0 . Se além disso, $\operatorname{Im} d_{n} \subseteq P_{n-1} \underline{\mathrm{r}}$ para todo $n \geq 1$, então a resolução acima é dita minimal.

No Capítulo 3 estudamos módulos que admitem resoluções projetivas graduadas onde o projetivo que ocupa a $i$-ésima posição da resolução é gerado em grau $i$, para todo $i \geq 0$. Uma resolução com esta propriedade é chamada de resolução linear.

Para as álgebras graduadas 0,1-split-básicas valem, para módulos graduados, alguns resultados análogos aos dos casos usuais para álgebras de dimensão finita . Por exemplo:

1. Um submódulo graduado $N$ de um módulo graduado $M$ finitamente gerado é supérfluo, no sentido graduado, se e somente se está contido no $\operatorname{radical} \operatorname{graduado} \operatorname{rad} M:=M \underline{\mathrm{r}}$ de $M$.

2. Um epimorfismo $f: L \rightarrow M$ na categoria de módulos graduados é supérfluo se e somente se seu núcleo está contido no radical graduado $\operatorname{rad} L$ de $L$.

3. O radical graduado $\operatorname{rad} M$ de um módulo graduado $M$ é a intersecção de todos os submódulos graduados maximais de $M$.

\footnotetext{
${ }^{4}$ Veja, por exemplo, [18].
} 


\section{Capítulo 2}

\section{Álgebras de Yoneda}

Neste capítulo estudaremos a estrutura da álgebra de Extensões, também chamada álgebra de Yoneda, de uma álgebra monomial. Num primeiro momento, construiremos uma base multiplicativa para a álgebra de Yoneda, veremos que essa construção nos permite dar uma boa descrição do seu carcás e com isso apresentamos o principal resultado do capítulo, que dá algumas características da álgebra de Yoneda de uma álgebra monomial quadrática. Ao final, como aplicação deste resultado, construiremos uma classe de álgebras que coincidem com sua álgebra de Yoneda. Este capítulo segue basicamente o que foi feito em [20].

\subsection{Introdução}

Começamos relembrando duas formas de olhar os elementos da álgebra de Yoneda. Aqui $\Lambda$ denota uma k-álgebra.

\section{Via resoluções projetivas}

Considere uma resolução projetiva para o $\Lambda$-módulo $M$ :

$$
(\mathcal{P}) \quad \cdots \longrightarrow P_{n} \stackrel{d_{n}}{\longrightarrow} \cdots \stackrel{d_{2}}{\longrightarrow} P_{1} \stackrel{d_{1}}{\longrightarrow} P_{0} \stackrel{d_{0}}{\longrightarrow} M \longrightarrow 0
$$

Fixando um $\Lambda$-módulo $N$ e aplicando o funtor $\operatorname{Hom}_{\Lambda}(-, N)$ à seqüência acima, obtemos o seguinte complexo

$$
0 \longrightarrow \operatorname{Hom}_{\Lambda}\left(P_{0}, N\right) \stackrel{d_{1}^{*}}{\longrightarrow} \operatorname{Hom}_{\Lambda}\left(P_{1}, N\right) \longrightarrow \cdots \stackrel{d_{n}^{*}}{\longrightarrow} \operatorname{Hom}_{\Lambda}\left(P_{n+1}, N\right) \longrightarrow \cdots
$$

onde $d_{i}^{*}:=\operatorname{Hom}_{\Lambda}\left(d_{i}, N\right): \operatorname{Hom}_{\Lambda}\left(P_{i-1}, N\right) \rightarrow \operatorname{Hom}_{\Lambda}\left(P_{i}, N\right)$ é definida por $d_{i}^{*}(f)=f \circ d_{i}$, para cada morfismo $f: P_{i-1} \rightarrow N$. Define-se então $\operatorname{Ext}_{\Lambda}^{n}(M, N):=\frac{\operatorname{Nuc}\left(d_{n+1}^{*}\right)}{\operatorname{Im} d_{n}^{*}}$, e dado $\xi \in$ Nuc $\left(d_{n+1}^{*}\right)$ denotaremos por $[\xi]$ sua classe em $\operatorname{Ext}_{\Lambda}^{n}(M, N)$.

Se $(\mathcal{P})$ e $(\mathcal{Q})$ são resoluções projetivas de $M$ e $N$, respectivamente, $[\eta] \in \operatorname{Ext}_{\Lambda}^{m}(M, N)$ e $[\xi] \in$ $\operatorname{Ext}_{\Lambda}^{n}(N, L)$, então temos o seguinte diagrama comutativo: 


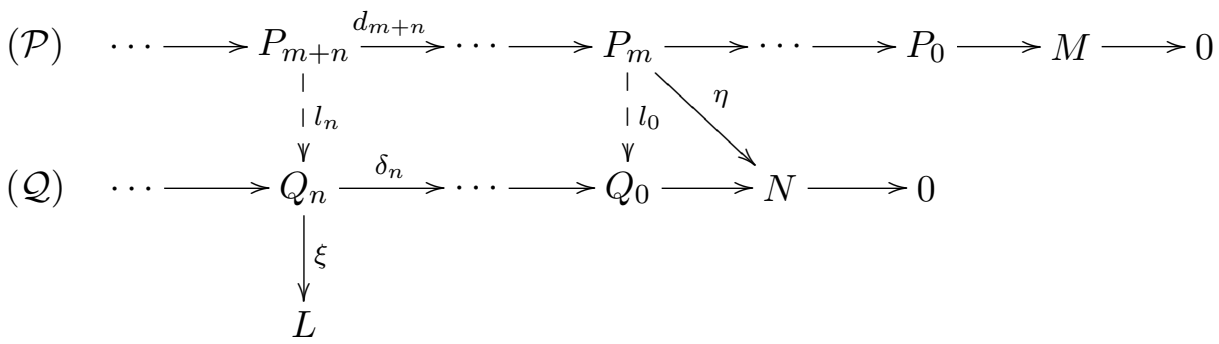

onde os $l_{i}$ 's denotam sucessivos "liftings" para $\eta$, obtidos no Teorema da Comparação (Teo. 1.6).

Define-se o produto de $[\xi]$ por $[\eta]$ como $[\xi] \cdot[\eta]:=\left[\xi \circ l_{n}\right]$. Observemos que, como $\xi \circ l_{n} \in$ $\operatorname{Hom}_{\Lambda}\left(P_{m+n}, L\right)$ e o diagrama acima é comutativo, então $d_{n+m+1}^{*}\left(\xi \circ l_{n}\right):=\xi \circ l_{n} \circ d_{m+n+1}=$ $\xi \circ \delta_{n+1} \circ l_{n+1}=\xi \circ 0=0$. Logo $\left[\xi \circ l_{n}\right] \in \operatorname{Ext}_{\Lambda}^{n+m}(M, L)$.

\section{Via extensões}

Sejam $M$ e $N$ dois $\Lambda$-módulos. Uma $n$-extensão de $M$ por $N$ é uma sequência exata de $\Lambda$-módulos

$$
\xi: 0 \rightarrow N \rightarrow M_{n-1} \rightarrow \cdots \rightarrow M_{0} \rightarrow M \rightarrow 0
$$

iniciando em $N$, terminando em $M$ e com $n$ termos intermediários.

Sejam $\xi: 0 \rightarrow N \rightarrow M_{n-1} \rightarrow \cdots \rightarrow M_{0} \rightarrow M \rightarrow 0$ e $\eta: 0 \rightarrow N \rightarrow M_{n-1}^{\prime} \rightarrow \cdots \rightarrow M_{0}^{\prime} \rightarrow M \rightarrow$ 0 duas $n$-extensões de $M$ por $N$. Dizemos que $\xi$ está relacionada com $\eta$, e escrevemos $\xi \rightsquigarrow \eta$, quando existir um diagrama comutativo da forma:

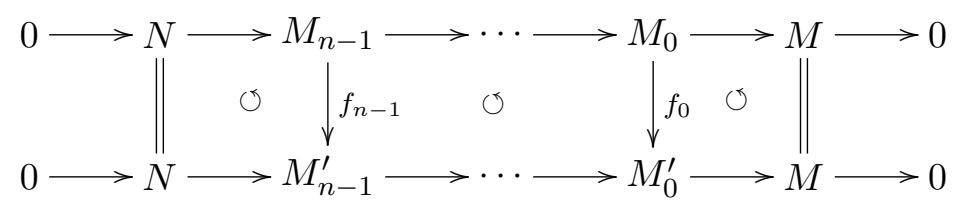

Observação 2.1 A relação “»” definida acima não é necessariamente simétrica.

A observação acima sugere a seguinte definição.

Dizemos que duas $n$-extensões $\xi$ e $\eta$ de $M$ por $N$, são equivalentes, e escrevemos $\xi \sim \eta$, se existe uma sequência de $n$-extensões de $M$ por $N$

$$
\xi=\xi_{0} ; \xi_{1} ; \ldots ; \xi_{n}=\eta
$$

tal que para cada $i=1, \ldots, n-1$, temos que $\xi_{i} \rightsquigarrow \xi_{i+1}$ ou $\xi_{i+1} \rightsquigarrow \xi_{i}$.

Assim, a relação " " é claramente uma relação de equivalência e o conjunto de todas as classes de equivalência de $n$-extensões de $M$ por $N$ será denotado por $\mathcal{E} \mathcal{X} \mathcal{T}_{\Lambda}^{n}(M, N)$ e seus elementos por $[\xi]$.

No que segue, definiremos o chamado Produto de Yoneda entre duas extensões quaisquer:

Se $\xi: 0 \rightarrow N \rightarrow M_{n-1} \rightarrow \cdots \rightarrow M_{0} \rightarrow M \rightarrow 0$ e $\eta: 0 \rightarrow M \rightarrow L_{m-1} \rightarrow \cdots \rightarrow L_{0} \rightarrow L \rightarrow 0$ representam elementos de $\mathcal{E} \mathcal{X} \mathcal{T}_{\Lambda}^{n}(M, N)$ e $\mathcal{E} \mathcal{X} \mathcal{T}_{\Lambda}^{m}(L, M)$, respectivamente, então considerando o 
diagrama

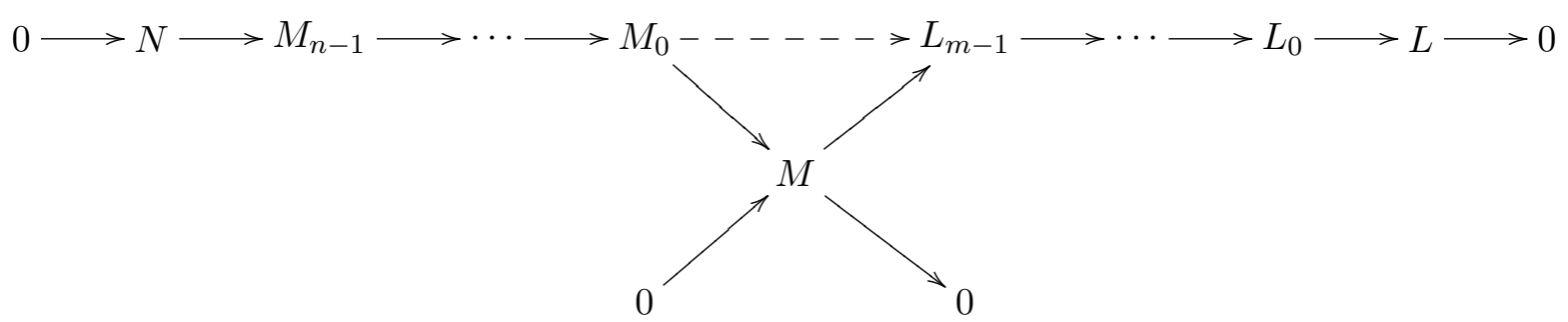

obtemos um elemento em $\mathcal{E X}^{\mathcal{T}} \mathcal{T}_{\Lambda}^{n+m}(L, N)$, denotado por $\xi \eta$. Assim, definimos o produto (de Yoneda) de $[\xi]$ por $[\eta]$ como sendo a classe de equivalência da extensão $\xi \eta$. Mais brevemente, $[\xi] \cdot[\eta]:=[\xi \eta]$.

Para maiores detalhes sobre as estruturas definidas acima, consulte por exemplo [8](2.4 e 2.6) ou [21] (Cap. 3), onde vemos também que essas duas formas são equivalentes. Por isso, a partir de agora não faremos distinção entre ambas, denotando por $\operatorname{Ext}_{\Lambda}^{n}(M, N)$ ambos os conjuntos e fazendo uso do produto mais apropriado em cada caso.

Finalmente, se $\Lambda$ é uma k-álgebra tal que $\Lambda / \underline{r}$ é um anel artiniano semisimples, então $E(\Lambda):=$ $\bigoplus_{n \geq 0} \operatorname{Ext}_{\Lambda}^{n}(\Lambda / \underline{r}, \Lambda / \underline{r})$ é a álgebra de Yoneda de $\Lambda$. Observe que

$$
\operatorname{Ext}_{\Lambda}^{m}(\Lambda / \underline{r}, \Lambda / \underline{r}) \cdot \operatorname{Ext}_{\Lambda}^{n}(\Lambda / \underline{r}, \Lambda / \underline{r}) \subseteq \operatorname{Ext}_{\Lambda}^{m+n}(\Lambda / \underline{r}, \Lambda / \underline{r})
$$

para todos $m, n \geq 0$. Em particular, $E(\Lambda)$ é uma álgebra graduada. Também, se $M$ é um $\Lambda$-módulo então $E(M):=\bigoplus_{n \geq 0} \operatorname{Ext}_{\Lambda}^{n}(M, \Lambda / \underline{r})$ é uma $E(\Lambda)$-módulo graduado, onde $E(M)_{j} \cdot E(\Lambda)_{k}$ é dado pelo produto de Yoneda.

\section{2 Álgebra de Yoneda de uma álgebra monomial}

Sejam $\Gamma$ um carcás finito e $\rho=\left\{\rho_{1}, \cdots, \rho_{n}\right\}$ um conjunto de caminhos em $\Gamma$ tal que nenhum $\rho_{i}$ é subcaminho de um outro $\rho_{j}$. Além disso, assumimos também que cada $\rho_{i}$ tem comprimento maior ou igual a dois. Seja $\Lambda=\mathrm{k} \Gamma / I$, onde $I=\langle\rho\rangle$. Lembramos que $\Gamma_{0}$ e $\Gamma_{1}$ denotam, respectivamente, os conjuntos dos vértices e das flechas de $\Gamma$. Definimos indutivamente conjuntos $\Gamma_{i}$, cuja união pode ser vista como uma k-base (multiplicativa) para $E(\Lambda)$.

Definição 2.1 Sejam $\Gamma_{2}:=\rho, \mathcal{B}$ a base usual de $\mathrm{k} \Gamma$ consistindo de todos os caminhos em $\Gamma$, $e$ $\mathcal{M}:=\{p \in \mathcal{B}:$ nenhum subcaminho de p pertence a $\rho\}$. Para $i \geq 2$, um caminho $p$ em $\mathrm{k} \Gamma e ́$ uma $i$-pré-cadeia se $p=q r s$, onde $q \in \Gamma_{i-1}$, qr $\in \Gamma_{i}, s \in \mathcal{M}-\Gamma_{0}$ e rs contém um subcaminho pertencente $a \Gamma_{2}$.

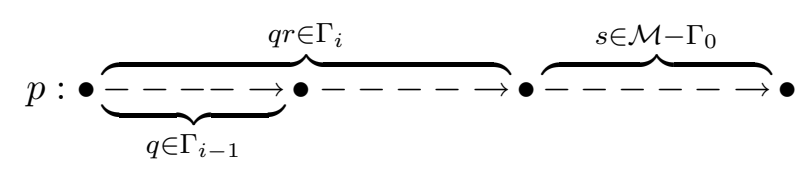

Dizemos que uma i-pré-cadeia é uma i-cadeia se nenhum subcaminho próprio inicial (ou à esquerda) é uma i-pré-cadeia. Definimos $\Gamma_{i+1}$ como o conjunto de todas as $i$-cadeias. 
Esta sequência de conjuntos é de suma importância, pois "descreve" uma resolução projetiva minimal do $\Lambda$-módulo $\bar{\Lambda}=\Lambda / \underline{r}$ da seguinte forma:

$$
(\mathcal{P}): \quad \cdots \rightarrow P_{n} \rightarrow \cdots \rightarrow P_{1} \rightarrow P_{0} \rightarrow \bar{\Lambda} \rightarrow 0
$$

com o $i$-ésimo projetivo dado por

$$
P_{i}=\bigoplus_{p \in \Gamma_{i}} e_{p} \Lambda
$$

onde $e_{p}$ é o idempotente correspondente ao caminho trivial $\mathrm{t}(p)$ (ver [15]). Sabendo que $\operatorname{Ext}_{\Lambda}^{i}(\bar{\Lambda}, \bar{\Lambda})=$

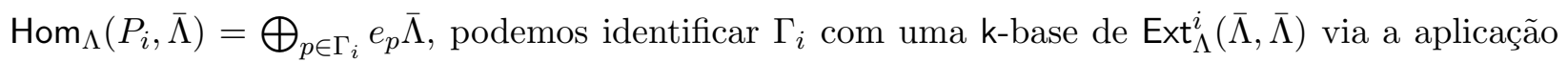
$\Gamma_{i} \ni p \longmapsto h_{p}^{i} \in \operatorname{Hom}_{\Lambda}\left(P_{i}, \bar{\Lambda}\right)$, onde

$$
h_{p}^{i}\left(e_{q} \lambda\right):=\left\{\begin{array}{rl}
0, & \text { se } p \neq q \text { em } \mathrm{k} \Gamma \\
e_{q} \bar{\lambda}, & \text { se } p=q \text { em } \mathrm{k} \Gamma
\end{array},\right.
$$

e $\bar{\lambda}:=\lambda+\underline{r}$.

O conjunto $\mathcal{G}_{i}:=\left\{h_{p}^{i}: p \in \Gamma_{i}\right\}$ é uma k-base para $\operatorname{Ext}_{\Lambda}^{i}(\bar{\Lambda}, \bar{\Lambda})$ e de $[20]$ temos que a união $\bigcup_{i \geq 0} \mathcal{G}_{i}$ forma uma base multiplicativa para $E(\Lambda)$, no sentido que para $h_{p}^{i} \in \mathcal{G}_{i}$ e $h_{q}^{j} \in \mathcal{G}_{j}$, temos que

$$
h_{p}^{i} h_{q}^{j}=\left\{\begin{array}{rl}
h_{p q}^{i+j}, & \text { se } p q \in \Gamma_{i+j} \\
0, & \text { caso contrário }
\end{array} .\right.
$$

Como consequência da discussão acima, temos a seguinte

Proposição 2.1 Sejam $\Gamma$ um carcás finito e $J$ o ideal de $\mathrm{k} \Gamma$ gerado pelas flechas de $\Gamma$. Então, para todo $n \geq 2$, temos que a álgebra $E\left(\mathrm{k} \Gamma / J^{n}\right)$ é finitamente gerada, com geradores em grau no máximo 2.

Prova.

Observemos inicialmente que por hipótese $\Gamma_{2}$ é igual ao conjunto dos caminhos de comprimento igual a $n$. Assim, se $p \in \Gamma_{3}$ então (por definição) $p=q r s$, onde $q \in \Gamma_{1}, q r \in \Gamma_{2}$ e $r s$ contém um subcaminho em $\Gamma_{2}$. Então $\ell(r)=n-1$ e, como $p$ não possui subcaminho inicial em $\Gamma_{3}, \ell(s)=1$. De onde segue que $p=q_{1} q_{2}$, onde $q_{1}:=q r \in \Gamma_{2}$ e $q_{2}:=s \in \Gamma_{1}$. Em particular, mostramos que os caminhos em $\Gamma_{2+1}$ têm comprimento $n+1$.

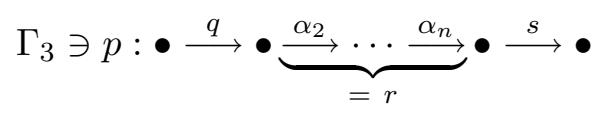

Analogamente, se $p \in \Gamma_{4}$, então temos a seguinte situação:

$$
p: \bullet \underbrace{\stackrel{q \in \Gamma_{2}}{\rightarrow} \bullet \rightarrow r}_{q r \in \Gamma_{3}} \rightarrow \stackrel{r}{\rightarrow} \bullet \stackrel{s \in \mathcal{M}-\Gamma_{0}}{\rightarrow} \bullet
$$


Como observado acima, $\ell(q r)=n+1$ e, portanto, $\ell(r)=1$. Assim, dado que $s \in \mathcal{M}-\Gamma_{0}$, segue as desigualdades $2 \leq \ell(r s) \leq n$. Mas como $r s$ contém um subcaminho de comprimento $n$, então $\ell(r s)=n$ e, portanto, $r s \in \Gamma_{2}$. Isto mostra que $p=q_{1} q_{2}$, onde $q_{1}:=q \in \Gamma_{2}$ e $q_{2}:=r s \in \Gamma_{2}$. Em particular, os caminhos pertencentes a $\Gamma_{2 \times 2}$ têm comprimento $2 n$.

Por indução, segue que os elementos de $\Gamma_{2 k+1}$ têm comprimento $k n+1$ e os elementos de $\Gamma_{2 k}$ têm comprimento $k n$, para todo $k \geq 1$. Logo,

$$
p=\left\{\begin{array}{cll}
q_{1} \cdots q_{k} \alpha, & \text { se } & p \in \Gamma_{2 k+1} \\
q_{1} \cdots q_{k}, & \text { se } & p \in \Gamma_{2 k}
\end{array},\right.
$$

onde $q_{i} \in \Gamma_{2}$ e $\alpha \in \Gamma_{1}$. Como $\Gamma_{0} \cup \Gamma_{1} \cup \Gamma_{2}$ é finito e $\bigcup \Gamma_{i}$ é uma k-base para $E\left(\mathrm{k} \Gamma / J^{n}\right)$ o resultado segue.

Na verdade em [17] este resultado é estendido no seguinte sentido:

- Seja $I$ um ideal monomial gerado por elementos, de um conjunto $\rho$, de comprimento $d \geq 2$. Então $E(\mathrm{k} \Gamma / I)$ é gerada em grau no máximo 2 se, e somente se, $\rho$ satisfaz a seguinte condição:

- se qrs é um caminho em $\Gamma$ com $q r, r s \in \rho$ e $\ell(r) \geq 1$, então todo subcaminho de qrs de comprimento $d$ está em $\rho$.

Exemplo 2.1 Seja $\Lambda=\mathrm{k} \Gamma /\langle\rho\rangle$, onde todos os caminhos em $\rho$ têm comprimento 2. Se $p \in \Gamma_{3}$, então $p=\alpha \beta$ s, onde $\alpha, \beta \in \Gamma_{1}, \alpha \beta \in \Gamma_{2}:=\rho e \beta s \in \Gamma_{2}$ (e assim $s \in \Gamma_{1}$ ).

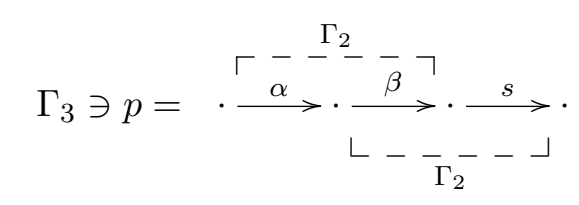

E se $p \in \Gamma_{4}$, então $p=q r s$, onde $q \in \Gamma_{2}(\Rightarrow q=\alpha \beta), q r \in \Gamma_{3}(\Rightarrow q r=\alpha \beta \gamma)$, rs $\in \Gamma_{2}$ (e assim $\left.s \in \Gamma_{1}\right)$.

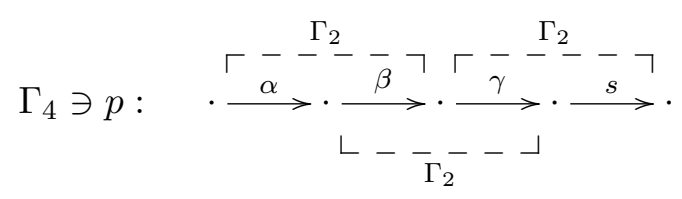

Por indução, segue a seguinte a forma para um elemento de $\Gamma_{n}, n \geq 5$ :

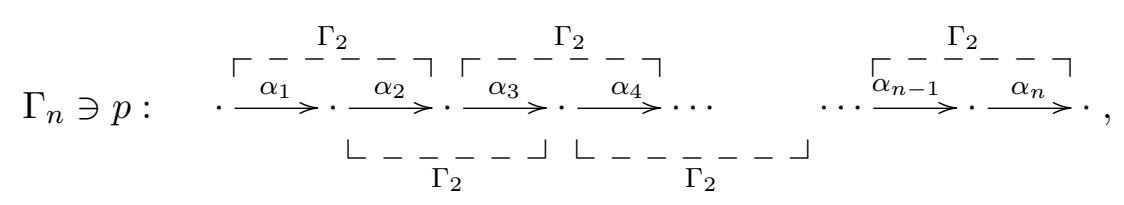

ou seja, se $p \in \Gamma_{n}$, então $p=\alpha_{1} \alpha_{2} \cdots \alpha_{n}$, onde para cada $1 \leq i \leq n-1, \alpha_{i} \alpha_{i+1} \in \Gamma_{2}$. Isto mostra que $E(\Lambda)$ é gerada em graus 0 e 1 , ou seja, se $\Lambda$ é uma álgebra monomial quadrática então $E(\Lambda)$ é gerada em graus 0 e $1 .^{1}$

\footnotetext{
${ }^{1}$ Veja o Teorema 2.3.
} 


\subsection{Carcás de $E(\Lambda)$}

Nessa seção $\Gamma$ é um carcás finito e $\Lambda=\mathrm{k} \Gamma / I$ uma álgebra monomial, como anteriormente.

No que segue, escreveremos a álgebra de Yoneda $E(\Lambda)$ como quociente de uma álgebra de caminhos. Para tanto, iniciamos com as seguintes convenções:

- fixe um subconjunto $Q \subseteq \bigcup_{i \geq 0} \Gamma_{i}$ tal que o subconjunto $\left\{h_{\bar{p}}\right\}_{\bar{p} \in Q}$ da k-base multiplicativa $\bigcup_{i \geq 0} \mathcal{G}_{i}:=\bigcup_{i \geq 0}\left\{h_{p}^{i}: p \in \Gamma_{i}\right\}$ de $E(\Lambda)$ seja um conjunto minimal de geradores de $E(\Lambda)$ como k-álgebra. Observe que tal conjunto sempre existe, pois a própria k-base $\bigcup_{i \geq 0} \mathcal{G}_{i}$ gera $E(\Lambda)$ como k-álgebra. Além disso, note que $\Gamma_{0} \cup \Gamma_{1} \subseteq Q$.

- $\Sigma$ é um conjunto de indeterminadas $\left\{x_{\bar{p}}\right\}$ que estão em correpondência biunívoca com o subconjunto $\left\{h_{\bar{p}}\right\}_{\bar{p}}$. Então obtemos uma função injetora $f: \Sigma \rightarrow \bigcup_{i \geq 0} \Gamma_{i}$ definida por $f\left(x_{\bar{p}}\right):=\bar{p}$ e, com isso, dizemos que $x_{\bar{p}}$ tem grau $i$ quando $\bar{p}$ for um elemento de $\Gamma_{i}$.

- $\Sigma_{0}:=\left\{x_{\bar{p}}: \bar{p} \in \Gamma_{0}\right\}$ e $\Sigma_{+}:=\left\{x_{\bar{p}}: \bar{p} \in Q \backslash \Gamma_{0}\right\}$. Então $\Sigma=\Sigma_{0} \cup \dot{U} \Sigma_{+}$e existe uma correspondência biunívoca entre $\Sigma_{0}$ e $\Gamma_{0}$.

Com estas convenções e observações, podemos definir um carcás associado a $E(\Lambda)$ como segue:

Definição 2.2 Seja $\Delta$ o carcás tendo como conjunto de vértices $\Delta_{0}:=\Sigma_{0}$ e como conjunto de flechas $\Delta_{1}:=\Sigma_{+}$. Mais precisamente, se $i$ e $j$ são dois vértices em $\Delta$, então existe uma flecha de $x_{\bar{q}}: i \rightarrow j$ se e somente se, no carcás $\Gamma$, existe um caminho não nulo $\bar{q}: f(i) \rightarrow \cdots \rightarrow f(j)$ tal que $\bar{q} \in Q$.

Do fato de $\Gamma_{1} \subseteq Q$, temos que se $\alpha$ é uma flecha em $\Gamma$, então $h_{\alpha}^{1}$ é um elemento do conjunto minimal $\left\{h_{\bar{p}}\right\}_{\bar{p} \in Q}$ e, portanto, existe único $x_{\alpha} \in \Sigma_{+}, x_{\alpha}: f^{-1}(o(\alpha)) \rightarrow f^{-1}(t(\alpha))$. De onde segue que $\Gamma$ é um subcarcás de $\Delta$.

Exemplo 2.2 (a) Seja $\Gamma$ o carcás $1 \underset{\beta}{\stackrel{\alpha}{\rightleftarrows}} 2, \rho=\{\alpha \beta \alpha\}$ e $\Lambda:=\mathrm{k} \Gamma /\langle\rho\rangle$. Então $\Gamma_{0}=\{1,2\}, \Gamma_{1}=$ $\{\alpha, \beta\}, \Gamma_{2}=\{\alpha \beta \alpha\}, \Gamma_{3}=\{\alpha \beta \alpha \beta \alpha\}, \ldots, \Gamma_{n+1}=\left\{(\alpha \beta)^{n} \alpha\right\}, \ldots$ Neste caso, $Q=\bigcup_{i \geq 0} \Gamma_{i}$ e o carcás $\Delta$ é dado por

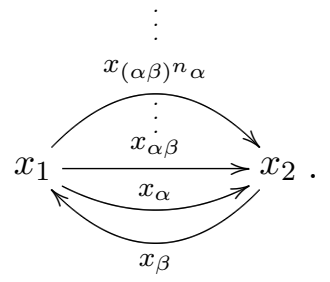

Em particular $E(\Lambda)$ não é finitamente gerada. 
(b) Seja $\Gamma$ o carcás do item (a), $\rho=\{\alpha \beta \alpha, \beta \alpha \beta\}$ e $\Lambda:=\mathrm{k} \Gamma /\langle\rho\rangle$. Então $\Gamma_{0}=\{1,2\}, \Gamma_{1}=$ $\{\alpha, \beta\}, \Gamma_{2}=\{\alpha \beta \alpha, \beta \alpha \beta\}, \Gamma_{3}=\{\alpha \beta \alpha \beta, \beta \alpha \beta \alpha\}, \Gamma_{4}=\{\alpha \beta \alpha \beta \alpha \beta, \beta \alpha \beta \alpha \beta \alpha\} \ldots, \Gamma_{n+1}=$ $\left\{(\alpha \beta)^{n},(\beta \alpha)^{n}\right\}, \ldots$

$$
\begin{aligned}
& \Gamma_{3}: \quad \cdot \stackrel{\Gamma}{\longrightarrow} \cdot \stackrel{\beta}{\longrightarrow} \cdot \stackrel{\alpha}{\longrightarrow} \cdot \stackrel{\beta}{\longrightarrow} . \\
& \Gamma_{4}: \quad \cdot \stackrel{\ulcorner\alpha}{\longrightarrow} \cdot \stackrel{\beta}{\llcorner-------\lrcorner} \stackrel{\longrightarrow}{\longrightarrow} \cdot \stackrel{\beta}{\longrightarrow} \cdot \stackrel{\alpha}{\longrightarrow} \cdot \stackrel{\beta}{\longrightarrow} .
\end{aligned}
$$

Observe que $E(\Lambda)$ é finitamente gerada (Proposição 2.1) e $Q=\Gamma_{0} \cup \Gamma_{1} \cup \Gamma_{2}$. De onde segue que o carcás de $E(\Lambda)$ tem a seguinte forma

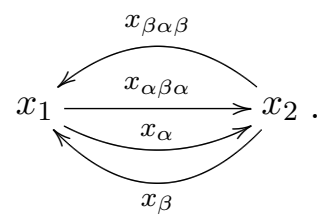

Seja $\Delta$ o carcás de $E(\Lambda)$ como na Definição 2.2. Considere a álgebra de caminho $\mathrm{k} \Delta$ e definamos $\psi: \mathrm{k} \Delta \rightarrow E(\Lambda)$ da seguinte forma:

- se $x_{p}$ é um vértice em $\Delta$, então $p$ é um vértice em $\Gamma$ e $\psi_{0}\left(x_{p}\right):=h_{p}$. Observe que, dados $p, q \in \Gamma_{0}$, então $x_{p}=x_{q}$ se e somente se $p=q$. Logo $\psi_{0}$ é função.

- se $x_{\bar{p}_{1}} \cdots x_{\bar{p}_{t}}$ é um caminho em $\Delta$, então $\psi_{1}\left(x_{\bar{p}_{1}} \cdots x_{\bar{p}_{t}}\right):=h_{\bar{p}_{1}} \cdots h_{\bar{p}_{t}}$. Note que $x_{\bar{p}_{1}} \cdots x_{\bar{p}_{s}}=$ $x_{\bar{q}_{1}} \cdots x_{\bar{q}_{t}}$ implica que $s=t$ (pois cada compontente do caminho é uma flecha) e $x_{\bar{p}_{i}}=x_{\bar{q}_{i}}$ para todo $i=1, \ldots, t$. Isto mostra que $h_{\bar{p}_{1}} \cdots h_{\bar{p}_{t}}=h_{\bar{q}_{1}} \cdots h_{\bar{q}_{t}}$ e, portanto, que $\psi_{1}$ é uma função.

- finalmente, definamos $\psi$ como sendo o homomorfismo de k-álgebra que extende $\psi_{0}$ e $\psi_{1}$.

Como $\left\{h_{\bar{p}}\right\}$ gera $E(\Lambda)$, então $\psi: \mathrm{k} \Delta \rightarrow E(\Lambda)$ é um epimorfismo entre k-álgebras, de onde segue que $E(\Lambda) \cong \mathrm{k} \Delta /$ Nuc $\psi$. A próxima proposição explicita o ideal Nuc $\psi$. Usaremos a seguinte notação: $\partial\left(\bar{p}_{1} \cdots \bar{p}_{n}\right) \doteq\left(\right.$ grau de $\left.x_{\bar{p}_{1}}\right)+\cdots+\left(\right.$ grau de $\left.x_{\bar{p}_{n}}\right)$.

Proposição 2.2 O Nuc $\psi$ é o ideal de $\mathrm{k} \Delta$ gerado pelos elementos da forma $x_{\bar{p}_{1}} \cdots x_{\bar{p}_{n}}$ com o caminho $\bar{p}_{1} \cdots \bar{p}_{n}$ não pertencente a $\Gamma_{\partial\left(\bar{p}_{1} \cdots \bar{p}_{n}\right)}$, e pelos elementos da forma $x_{\bar{p}_{1}} \cdots x_{\bar{p}_{n}}-x_{\bar{q}_{1}} \cdots x_{\bar{q}_{m}}$ com $\bar{p}_{1} \cdots \bar{p}_{n}=\bar{q}_{1} \cdots \bar{q}_{m}$ e $\partial\left(\bar{p}_{1} \cdots \bar{p}_{n}\right)=\partial\left(\bar{q}_{1} \cdots \bar{q}_{m}\right)$.

Prova.

Se $x_{\bar{p}_{1}} \cdots x_{\bar{p}_{n}}$ é um caminho em k $\Delta$ tal que $\bar{p}_{1} \cdots \bar{p}_{n} \notin \Gamma_{\partial\left(\bar{p}_{1} \cdots \bar{p}_{n}\right)}$ então, pela definição das $h_{q}^{i}$ 's, temos que $h_{\bar{p}_{1}} \cdots h_{\bar{p}_{t}}=0$ em $E(\Lambda)$, e portanto, $x_{\bar{p}_{1}} \cdots x_{\bar{p}_{n}} \in$ Nuc $\psi$. E se $x_{\bar{p}_{1}} \cdots x_{\bar{p}_{n}}-x_{\bar{q}_{1}} \cdots x_{\bar{q}_{m}}$ é 
um caminho em k $\Delta$ tal que $\bar{p}_{1} \cdots \bar{p}_{n}=\bar{q}_{1} \cdots \bar{q}_{m}$ e $\partial\left(\bar{p}_{1} \cdots \bar{p}_{n}\right)=\partial\left(\bar{q}_{1} \cdots \bar{q}_{m}\right)$, então

$$
\begin{aligned}
\psi\left(x_{\bar{p}_{1}} \cdots x_{\bar{p}_{n}}\right) & :=h_{\bar{p}_{1} \cdots h_{\bar{p}_{n}}} \\
& =\left\{\begin{aligned}
h_{\bar{p}_{1} \cdots \bar{p}_{n}}, & \text { se } \bar{p}_{1} \cdots \bar{p}_{n} \in \Gamma_{\partial\left(\bar{p}_{1} \cdots \bar{p}_{n}\right)} \\
0, & \text { caso contrário }
\end{aligned}\right. \\
& =\left\{\begin{aligned}
h_{\bar{q}_{1} \cdots \bar{q}_{n}}, & \text { se } \left.\bar{q}_{1} \cdots \bar{q}_{n} \in \Gamma_{\partial\left(\bar{q}_{1} \cdots \bar{q}_{n}\right)}\right) \\
0, & \text { caso contrário } \\
& =h_{\bar{q}_{1}} \cdots h_{\bar{q}_{n}} \\
& :=\psi\left(x_{\bar{q}_{1}} \cdots x_{\bar{q}_{n}}\right) .
\end{aligned}\right.
\end{aligned}
$$

Logo, $x_{\bar{p}_{1}} \cdots x_{\bar{p}_{n}}-x_{\bar{q}_{1}} \cdots x_{\bar{q}_{n}}$ é um elemento do núcleo de $\psi$. Restá-nos mostrar que o Nuc $\psi$ é um subconjunto do ideal de $\mathrm{k} \Delta$ gerado pelos elemetos da forma enunciada. Para tanto, considere $\alpha_{i}$ 's como sendo escalares não nulos e $u_{i}$ 's caminhos em $\mathrm{k} \Delta$ tal que $\sum \alpha_{i} u_{i} \in$ Nuc $\psi$. Então $\sum \alpha_{i} \psi\left(u_{i}\right)=0$ em $E(\Lambda)$. Assim podemos supor que cada $u_{i}$ não pertecence ao Nuc $\psi$. Portanto, para cada $i, \psi\left(u_{i}\right)=h_{p_{i}}$, onde $p_{i}$ é algum caminho em $\Gamma_{0} \cup \Gamma_{1} \cup \ldots$ Mas dado que $\left\{h_{p}\right\}$ é uma k-base para $E(\Lambda)$, então cada $\alpha_{i}=0$, o que é um absurdo. Portanto, os elementos não nulos pertencentes ao núcleo de $\psi$ são caminhos de $\mathrm{k} \Delta$. Seja $x_{\bar{p}_{1}} \cdots x_{\bar{p}_{n}}$ um caminho em $\mathrm{k} \Delta$ tal que $\psi\left(x_{\bar{p}_{1}} \cdots x_{\bar{p}_{n}}\right)=0$. Logo, $h_{\bar{p}_{1}} \cdots h_{\bar{p}_{n}}=0$ em $E(\Lambda)$, o que implica que $\bar{p}_{1} \cdots \bar{p}_{n} \notin \Gamma_{\partial\left(\bar{p}_{1} \cdots \bar{p}_{n}\right)}$.

Passaremos a estudar álgebras monomiais que são dadas por relações quadrátricas. Na Proposição 2.1 mostramos que se o conjunto das relações de uma álgebra monomial possui todos os caminhos de um determinado comprimento, então a álgebra de Yoneda desta álgebra é gerada em grau no máximo 2. O próximo teorema garante que a álgebra de Yoneda, de álgebra monomial $\Lambda$, é gerada em grau 0 e 1, sempre que as relações de $\Lambda$ têm comprimento 2.

Teorema 2.3 Sejam $\Lambda$ uma álgebra monomial dada por relações quadrátricas, $\mathcal{M}_{2}$ o conjunto de todos os caminhos de comprimento 2 (no carcás de $\Lambda$ ) e $\mathcal{R}$ o conjunto de relações de $\Lambda$. Então:

(a) $E(\Lambda)$ é finitamente gerada com todos os geradores gerados em grau 1.

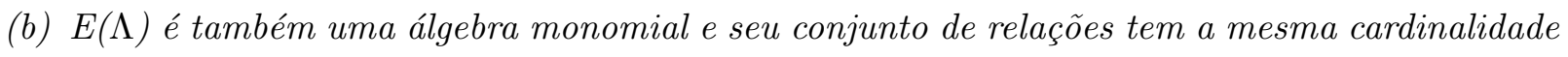
do conjunto $\mathcal{M}_{2} \backslash \mathcal{R}$.

(c) $\Lambda$ e $E(E(\Lambda))$ são isomorfas como k-álgebras.

Prova.

Seja $\Gamma$ o carcás de $\Lambda$.

(a) Seja $\mathcal{A} \subseteq \Gamma_{1}$ o conjunto das flechas em $\Gamma$ que forma (por composição) as relações em $\mathcal{R}$. Se $p$ é um caminho em $\Gamma$ tal que $p \in \Gamma_{n}$, para algum $n \geq 2$, então $p$ é uma composição de $n$ flechas de $\mathcal{A}$, dado que todas as relações de $\mathcal{R}$ têm comprimento 2 (veja Exemplo 2.1). Portanto, como $\left\{h_{p}^{i}\right\}_{p}$ é uma k-base multiplicativa para $E(\Lambda)$, segue que $E(\Lambda)$ finitamente gerada, como k-álgebra, e $\left\{h_{p}^{i}\right\}_{p \in \Gamma_{0} \cup \Gamma_{1}}$ é um sistema de geradores. 
(b) Do item (a), segue que o carcás $\Delta$ de $E(\Lambda)$ e o carcás $\Gamma$ são iguais. Se $a, b$ são flechas tais que $\mathrm{t}(a)=\mathrm{o}(b)$, então $x_{a} x_{b} \in \mathrm{Nuc} \psi$ se, e somente se, $a b \notin \Gamma_{2}$. Como $\Gamma_{2}:=\mathcal{R}$, então $x_{a} x_{b} \in$ Nuc $\psi$ se, e somente se, $a b \in \mathcal{M}_{2} \backslash \mathcal{R}$. Mas, dado que $\Delta=\Gamma$, temos que o conjunto $\left\{x_{a} x_{b}: a b \in \mathcal{M}_{2} \backslash \mathcal{R}\right.$ e $\left.a, b \in \Gamma_{1}\right\}$ gera o Nuc $\psi$. O resultado segue da proposição acima.

(c) Basta aplicar os itens (a) e (b) a álgebra $E(\Lambda)$.

Exemplo 2.3 (a) Seja $\Lambda=\mathrm{k} \Gamma$ a álgebra de caminhos do carcás finito $\Gamma$. Então $\mathcal{R}=\emptyset$ e portanto $E(\Lambda) \cong \mathrm{k} \Gamma / J^{2}$, onde $J$ é o ideal de $\mathrm{k} \Gamma$ gerado pelas flechas de $\Gamma$.

(b) Seja $\Lambda=\mathrm{k} \Gamma / J^{2}$. Então $\mathcal{R}=\mathcal{M}_{2}$ e $E(\Lambda) \cong \mathrm{k} \Gamma$. Em particular, se $\Gamma$ é o carcás $\mathrm{G} \circlearrowleft y$ e $\Lambda=\mathrm{k} \Gamma / I$, onde $I=\left\langle x^{2}, x y, y x, y^{2}\right\rangle$, então $E(\Lambda)=\mathrm{k}\langle x, y\rangle$ é a álgebra livre nas variáveis $x$ e $y$.

Observe que desse modo temos uma relação, até certo ponto inesperada, entre uma álgebra de dimensão finita com dimensão global infinita e uma álgebra hereditária de dimensão infinita.

\subsection{Uma construção de álgebras homologicamente auto-duais}

Dedicaremos esta seção à construção de álgebras que são isomorfas a sua álgebra de Yoneda. O principal resultado para esta construção segue como uma aplicação direta do que foi feito na seção anterior, mais especificamente do Teorema 2.3(b).

Definição 2.3 Uma k-álgebra $\Lambda$ é dita homologicamente auto-dual quando é isomorfa, como k-álgebra, à $E(\Lambda)$.

Consideremos $\Gamma$ um carcás finito, $G$ um grupo e $W: \Gamma_{1} \rightarrow G$ uma função, onde $\Gamma_{1}$ é o conjunto das flechas em $\Gamma$. Construímos um novo carcás $\Gamma_{W}$ da seguinte forma:

- $\left(\Gamma_{W}\right)_{0}:=\Gamma_{0} \times G$

- $\left(\Gamma_{W}\right)_{1}:=\{(a, g):(u, g) \rightarrow(v, g \cdot W(a)) \mid a: u \rightarrow v$ é uma flecha em $\Gamma$ e $g \in G\}$

Observe que um caminho de comprimento 2 em $\Gamma_{W}$ é da forma $p=(a, g)(b, g \cdot W(a))$, onde $a: u \rightarrow v, b: v \rightarrow w$ e $g \in G$.

Dado $h \in G$ definimos uma ação de $G$ em $\Gamma_{W}$ por $h \cdot(x, g):=(x, g \cdot h)$, onde $x$ é um elemento da união $\left(\Gamma_{W}\right)_{0} \cup\left(\Gamma_{W}\right)_{1}$. Tal ação induz uma bijeção $\phi_{h}: \Gamma_{W} \rightarrow \Gamma_{W}$ dada por $(x, g) \mapsto(x, g \cdot h)$, com inversa $\phi_{h}{ }^{-1}: \Gamma_{W} \rightarrow \Gamma_{W}$ dada por $(x, g) \mapsto\left(x, g \cdot h^{-1}\right)$. Consequentemente, existe um automorfismo de álgebras $\Phi_{h}: \mathrm{k} \Gamma_{W} \rightarrow \mathrm{k} \Gamma_{W}$ definido por $\Phi_{h}\left(\left(x_{1}, g_{1}\right) \ldots\left(x_{n}, g_{n}\right)\right):=\phi_{h}\left(x_{1}, g_{1}\right) \ldots \phi_{h}\left(x_{n}, g_{n}\right) \mathrm{e}$ estendido linearmente.

Um ponto crucial para nossa construção de álgebras homologicamente auto-duais é escolher "bem" caminhos de comprimento 2 no carcás $\Gamma_{W}$ definido acima. Para isto, suponhamos que $G$ seja um grupo (aditivo) cíclico de ordem par gerado por $h \in G$. Seja $X$ um conjunto de caminhos de comprimento 2 em $\Gamma$, escolhido arbitrariamente e defina $X_{W}$ como sendo o conjunto de caminhos $(a, g)(b, g+W(a))$ de comprimento 2 em $\Gamma_{W}$ tais que 
- $a b \in X$ e $g=n h$ para algum $n$ par; ou

- $a b \notin X$ e $g=n h$ para algum $n$ ímpar.

Note que para definição de $X_{W}$ é necessário que o grupo $G$ tenha ordem par. O lema abaixo mostra uma relação importante entre os elementos de $X_{W}$ e o automorfismo $\Phi_{h}$.

Lema 2.4 Seja $p \in \Gamma_{W}$ um caminho de comprimento 2. Então $p \in X_{W}$ se e somente se $\Phi_{h}(p) \notin$ $X_{W}$.

Prova.

Sejam $a, b \in \Gamma_{1}$ e $g=n h \in G$ tais que $p=(a, g)(b, g+W(a))$. Assim,

$$
\Phi_{h}(p)=(a, g+h)(b, g+h+W(a)) \text { e } g+h=(n+1) h .
$$

$(\Rightarrow)$ se $n$ é par então $a b \in X$. Logo $\Phi_{h}(p) \notin X_{W}$, pois $a b \in X$ e $n+1$ é ímpar.

De forma análoga para $n$ ímpar.

$(\Leftarrow)$ se $a b \in X$ temos que $n+1$ é ímpar e então $n$ é par. Logo $p \in X_{W}$.

De forma análoga para $a b \notin X$.

Com o resultado acima e o Teorema 2.3, estamos aptos a provar o principal resultado da seção.

Teorema 2.5 Sejam $\Gamma$ um carcás finito, $G$ um grupo cíclico de ordem par, $W: \Gamma_{1} \rightarrow G$ uma função e $X_{W}$ o conjunto definido anteriormente. Se I é o ideal monomial de $\mathrm{k}_{W}$ gerado por $X_{W}$, então a álgebra $\Lambda=\mathrm{k}_{W} / I$ é homologicamente auto-dual.

Prova.

Consideremos $X_{W}^{\prime}=\left\{\right.$ caminhos de comprimento 2 em $\left.\Gamma_{W}\right\} \backslash X_{W}$. Temos então que $E\left(\mathrm{k} \Gamma_{W} / I\right) \cong$ $\mathrm{k} \Gamma_{W} /\left\langle X_{W}^{\prime}\right\rangle$.

Vamos mostrar que existe um automorfismo $\Psi: \mathrm{k} \Gamma_{W} \rightarrow \mathrm{k} \Gamma_{W}$ tal que $\Psi\left(\left\langle X_{W}\right\rangle\right)=\left\langle X_{W}^{\prime}\right\rangle$, o qual induzirá um isomorfismo

$$
\bar{\Psi}: \frac{\mathrm{k} \Gamma_{W}}{\left\langle X_{W}\right\rangle} \longrightarrow \frac{\mathrm{k} \Gamma_{W}}{\left\langle X_{W}^{\prime}\right\rangle}
$$

Daí seguirá que

$$
\Lambda=\frac{\mathrm{k} \Gamma}{I}=\frac{\mathrm{k} \Gamma_{W}}{\left\langle X_{W}\right\rangle} \cong \frac{\mathrm{k} \Gamma_{W}}{\left\langle X_{W}^{\prime}\right\rangle} \cong E(\Lambda) .
$$

De fato, seja $\Psi=\Phi_{h}$ onde $h \in G$ é um gerador de $G$, pois segue do Lema 2.4 que $\Phi_{h}\left(\left\langle X_{W}\right\rangle\right)=$ $\left\langle X_{W}^{\prime}\right\rangle$. 
Exemplo 2.4 Consideremos $\Gamma$ o carcás $\underset{r_{c}}{\stackrel{a}{\longrightarrow}} v, G=\mathbb{Z}_{2}$, a função $W: \Gamma_{1} \rightarrow \mathbb{Z}_{2}$ definida por $W(a)=W(b)=0$ e $W(c)=1$ e o conjunto $X=\{a c\}$.

$O$ carcás $\Gamma_{W}$ é dado por

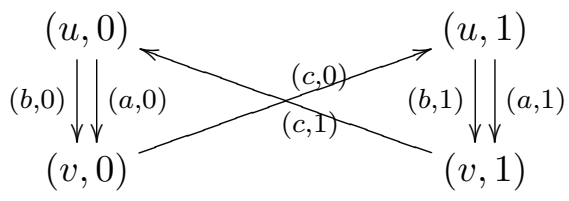

$X_{W}=\{(a, 0)(c, 0) ;(b, 1)(c, 1) ;(c, 1)(a, 0) ;(c, 1)(b, 0)\}$ e $\mathrm{k} \Gamma_{W} /\left\langle X_{W}\right\rangle$ é homologicamente auto-dual.

Finalizaremos a seção com a questão deixada por Green e Zacharia em [20]:

Suponha que $\frac{\mathrm{k} \Gamma}{I}$ é uma álgebra (monomial) homologicamente auto-dual. Será que existem um carcás $\bar{\Gamma}$, um número par $n$, uma função $W: \bar{\Gamma}_{1} \rightarrow \mathbb{Z}_{n}$ e um conjunto $X$ de caminhos de comprimento 2 em $\bar{\Gamma}$, tais que $\Gamma=\bar{\Gamma}_{W}$ e $I=\left\langle X_{W}\right\rangle$ ?

Observe que como $n$ é um número par, uma resposta positiva para essa questão implica que o carcás de toda ágebra homologicamente auto-dual tem um número par de vértices, pois pela contrução acima $\Gamma_{0}=\left(\bar{\Gamma}_{W}\right)_{0}=\bar{\Gamma}_{0} \times \mathbb{Z}_{n}$. Porém o próximo exemplo mostra que isso não é verdade em geral.

Exemplo 2.5 Consideremos a álgebra de caminhos dada pelo carcás $G_{9} \bigcirc$ y e pelo conjunto de relações $\rho=\left\{x^{2}, x y\right\}$. Temos que

$$
E\left(\frac{\mathrm{k} \Gamma}{\left\langle x^{2}, x y\right\rangle}\right) \cong \frac{\mathrm{k} \Gamma}{\left\langle y^{2}, y x\right\rangle} \cong \frac{\mathrm{k} \Gamma}{\left\langle x^{2}, x y\right\rangle}
$$

onde o primeiro isomorfismo é dado pelo Teorema 2.3 e o segundo é dado por $x \mapsto y$ e $y \mapsto x$. Portanto essa é uma álgebra homologicamente auto-dual cujo carcás tem um número ímpar de vértices.

O que naturalmente nos perguntamos agora é se a questão proposta acima é válida no caso em que o número de vértices de $\Gamma$ é um número par.

A resposta continua negativa nesse caso, como nos mostra o exemplo abaixo.

Exemplo 2.6 Consideremos a álgebra de Kronecker, isto é, a álgebra de caminhos dada pelo carcás $\Gamma: \bullet \underset{\beta}{\stackrel{\alpha}{\longrightarrow}}$ e cujo conjunto de relações é vazio. Observe que o conjunto $\mathcal{M}_{2}$ dos caminhos de comprimento 2 em $\Gamma$ é também vazio. Portanto $E(\mathrm{k} \Gamma) \cong \mathrm{k} \Gamma$, ou seja, a ágebra de Kronecker é homologicamente auto-dual. 
Suponhamos que existam um carcás $\bar{\Gamma}$ e um número par $n$ tais que $\Gamma_{0}=\bar{\Gamma}_{0} \times \mathbb{Z}_{n}$. Como o menor inteiro par é 2 então a cardinalidade de $\bar{\Gamma}_{0}$ deve ser 1 , digamos $\bar{\Gamma}_{0}=\{u\}$. Então $\Gamma_{0}=$ $\{(u, 0) ;(u, 1)\}$.

Como a cardinalidade de $\Gamma_{1}$ é igual a 2 então existe uma flecha a em $\bar{\Gamma}_{1}$. Suponhamos que $W(a)=1$, então temos as seguintes flechas em $\Gamma_{1}$ :

- $(a, 0):(u, 0) \rightarrow(u, 0+W(a))=(u, 1)$

- $(a, 1):(u, 1) \rightarrow(u, 1+W(a))=(u, 0)$

ou seja, $(u, 0) \underset{(a, 1)}{\stackrel{(a, 0)}{\longrightarrow}}(u, 1)$ seria um subcarcás de $\Gamma$, o que nãa acontece. Se $W(a)=0$, então teríamos a flecha $(a, 0):(u, 0) \rightarrow(u, 0+W(a))=(u, 0)$, ou seja, $\bigcap_{(u, 0)}^{(a, 0)}$ seria um subcarcás de $\Gamma$, o que também não acontece. Portanto não existe uma função $W: \bar{\Gamma}_{1} \rightarrow \mathbb{Z}_{2}$ que satisfaça as condições do teorema.

Deixaremos uma questão:

Toda álgebra homologicamente auto-dual é uma álgebra monomial? 


\section{Capítulo 3}

\section{Álgebras de Koszul}

No capítulo anterior estudamos a álgebra de Yoneda de uma álgebra monomial. Neste capítulo faremos um estudo um pouco mais geral, considerando álgebras graduadas. Um caso particular dessas álgebras, as chamadas álgebras de Koszul, será destacado em grande parte do capítulo, assim como a relação delas com sua respectiva álgebra de extensões. Veremos algumas relações entre álgebras quadráticas e álgebras de Koszul e finalizaremos o capítulo com uma caracterização destas álgebras quando olhadas como um módulo sobre sua álgebra envolvente.

O desenvolvimento deste capítulo segue basicamente $[18,26]$. Usaremos também alguns resultados de [19] e de [4].

A menos de menção em contrário, $k$ denotará um corpo, $\Gamma$ um carcás finito e $\mathrm{k} \Gamma$ a álgebra de caminhos de $\Gamma$ sobre $\mathrm{k}$. Olharemos para $\mathrm{k} \Gamma$ como uma álgebra graduada, onde um caminho de comprimento $n$ é um elemento homogênio de grau $n$, e $J=\bigoplus_{n \geq 1}(\mathrm{k} \Gamma)_{n}$ sendo o radical de Jacobson graduado. Além disso, para um dado ideal graduado $I$ de $\mathrm{k} \Gamma$, contido em $J^{2}$, denotaremos $\mathrm{k} \Gamma / I$ por $\Lambda$. Como vimos antes, $\Lambda$ é uma álgebra graduada 0,1 -split-básica e qualquer álgebra graduada 0,1 -split-básica tem essa forma. Neste caso, $\underline{r}=J / I$ é o radical de Jacobson graduado de $\Lambda$.

\subsection{Resoluções Lineares}

Iniciaremos com resoluções lineares.

Definição 3.1 (Resolução Linear) Seja $M$ um $\Lambda$-módulo graduado finitamente gerado. Suponha que $P_{n} \rightarrow P_{n-1} \rightarrow \cdots \rightarrow P_{1} \rightarrow P_{0} \rightarrow M \rightarrow 0$ é uma resolução projetiva graduada de $M$. Dizemos que esta resolução é linear de comprimento $n$ se para todo $0 \leq i \leq n, P_{i}$ é gerado em grau $i$. Por outro lado, dizemos que $M$ tem uma resolução linear se existe uma resolução projetiva graduada

$$
\cdots \rightarrow P_{n} \rightarrow P_{n-1} \rightarrow \cdots \rightarrow P_{1} \rightarrow P_{0} \rightarrow M \rightarrow 0
$$

que é linear para cada $n \geq 0$.

O próximo resultado mostra, em particular, que toda resolução linear é minimal (no sentido graduado). 
Lema 3.1 Sejam $\Lambda=\mathrm{k} \Gamma / I$, para algum ideal graduado $I$, e $M \in \operatorname{gr} \Lambda$ gerado em grau 0 . Se

$$
P_{n} \stackrel{d_{n}}{\longrightarrow} P_{n-1} \stackrel{d_{n-1}}{\longrightarrow} \cdots \stackrel{d_{1}}{\longrightarrow} P_{0} \stackrel{d_{0}}{\longrightarrow} M \longrightarrow 0
$$

é uma resolução linear (de comprimento $n$ ), então Nuc $\left(d_{i}\right) \subseteq P_{i} \underline{\underline{r}}$ e Nuc $\left(d_{i}\right) \underline{r}=P_{i} \underline{\underline{r}}^{2} \cap \operatorname{Nuc}\left(d_{i}\right)$, para todo $1 \leq i \leq n$.

Prova.

Suponha que $(*)$ é linear. Então para todo $0 \leq i \leq n, P_{i}$ é gerado em grau $i$. Mas como $d_{i+1}\left(P_{i+1}\right)=$ Nuc $\left(d_{i}\right)$, segue que Nuc $\left(d_{j}\right)$ é gerado em grau $j+1$, para todo $0 \leq j \leq n-1$. Por outro lado, dado que $P_{j}$ é gerado em grau $j$, então os elementos de grau $j+1$ estão em $P_{j} \underline{\text { e }}$ e, portanto, Nuc $\left(d_{j}\right) \subseteq P_{j} \underline{r}$. Analogamente, Nuc $\left(d_{j}\right) \underline{r} \subseteq \operatorname{Nuc}\left(d_{j}\right) \cap P_{j} \underline{r}^{2}$. Para a outra inclusão, seja $x \in \operatorname{Nuc}\left(d_{j}\right) \cap P_{j} \underline{r}^{2}$ um elemento homogêneo de grau $i$. Observe que $i \geq j+2$. Se $x \notin$ Nuc $\left(d_{j}\right) \underline{r}$, então $x$ é um gerador de Nuc $\left(d_{j}\right)$, que é gerado em grau $j+1$, contradizendo assim o fato de $i \geq j+2$.

Talvez seja interessante chamar a atenção do leitor para o fato de que a recíproca do lema acima também é verdadeira, isto é, se $(*)$ é uma resolução projetiva graduada de $M$ tal que Nuc $\left(d_{i}\right) \subseteq P_{i} \underline{r}$ e Nuc $\left(d_{i}\right) \underline{r}=P_{i} \underline{r}^{2} \cap \operatorname{Nuc}\left(d_{i}\right)$, para todo $1 \leq i \leq n$, então $(*)$ é linear. ${ }^{1}$

Proposição 3.2 Sejam $\Lambda=\mathrm{k} \Gamma / I$ e $M$ como no lema acima. Uma resolução projetiva

$$
P_{n} \stackrel{d_{n}}{\longrightarrow} P_{n-1} \stackrel{d_{n-1}}{\longrightarrow} \cdots \stackrel{d_{1}}{\longrightarrow} P_{0} \stackrel{d_{0}}{\longrightarrow} M \longrightarrow 0
$$

é linear se e somente se:

- $P_{i}=\bigoplus_{l \geq 0} e_{i_{l}} \Lambda(-i)$, para todo $0 \leq i \leq n$;

- a componente de $d_{i}\left(e_{i_{l}}\right)$ em algum somando $e_{(i-1)_{m}} \Lambda$ de $P_{i-1}$ está em $\Lambda_{1}$, para todo $0 \leq i \leq n$;

Prova.

$(\Rightarrow)$ A igualdade $P_{i}=\bigoplus_{l \geq 0} e_{i_{l}} \Lambda(-i)$ segue diretamente do fato de $P_{i}$ ser um módulo projetivo graduado gerado em grau $i$. Por outro lado, como cada $d_{i}$ é um morfismo de grau 0 , segue que $d_{i}\left(e_{i_{l}}\right) \in\left(P_{i-1}\right)_{i}$. Como $P_{i-1}$ é gerado em grau $i-1$, então os elementos de $\left(P_{i-1}\right)_{i}$ estão em $\left(P_{i-1}\right)_{i-1} \Lambda_{1}$ e, portanto, a componente de $d_{i}\left(e_{i_{l}}\right)$ em algum somando $e_{(i-1)_{m}} \Lambda$ de $P_{i-1}$ está em $\Lambda_{1}$.

$(\Leftarrow)$ Cada $P_{i}$ é um módulo projetivo graduado, gerado em grau $i$, pois cada somando $e_{i_{l}} \Lambda(-i)$ possui a mesma propriedade. Falta mostrar que $d_{i}\left(\left(P_{i}\right)_{m}\right) \subseteq\left(P_{i-1}\right)_{m}$, para todo $m$ e para todo $i$. Para isso, observe que

$$
\left(P_{i}\right)_{m}=\bigoplus_{l} e_{i_{l}} \Lambda_{m-i} \quad \text { e }\left(P_{i-1}\right)_{m}=\bigoplus_{t} e_{i_{t}} \Lambda_{m-i+1}
$$

\footnotetext{
${ }^{1}$ Veja Prop. 3.1 de [18].
} 
e por hipótese $d_{i}\left(e_{i_{l}}\right) \in \bigoplus_{t} e_{(i-1)_{t}} \Lambda_{1}$, de onde segue o resultado.

Observe que o segundo item da proposição acima diz que as entradas da matriz, que representa cada homomorfismo $d_{i}$, estão sempre em $\Lambda_{1}$.

O próximo resultado resume mais algumas propriedades básicas de resoluções lineares.

Corolario 3.3 Sejam $\Lambda=\mathrm{k} \Gamma / I$ uma álgebra graduada e $M$ um $\Lambda$-módulo com resolução linear

$$
\cdots \longrightarrow P_{n} \stackrel{d_{n}}{\longrightarrow} P_{n-1} \stackrel{d_{n-1}}{\longrightarrow} \cdots \stackrel{d_{1}}{\longrightarrow} P_{0} \stackrel{d_{0}}{\longrightarrow} M \longrightarrow 0
$$

Então $M$ é gerado em grau 0 , Nuc $\left(d_{i}\right)(i+1)$ tem resolução linear e, para cada $i \geq 0$, o núcleo de $d_{i}: P_{i} \rightarrow P_{i-1}$ é gerado em grau $i+1$.

\section{Prova.}

Como Nuc $\left(d_{i}\right)=\operatorname{Im}\left(d_{i+1}: P_{i+1} \rightarrow P_{i}\right)$, toda resolução linear é minimal e cada $P_{i}$ é gerado em grau $i$, então Nuc $\left(d_{i}\right)$ é gerado em grau $i+1$. Para ver que $M$ é gerado em grau 0 , basta lembrar que cada $d_{i}$ preserva graus e que $\operatorname{Im}\left(d_{0}\right)=M$. Finalmente,

$$
\cdots \longrightarrow P_{n}(i+1) \longrightarrow \cdots \longrightarrow P_{i+1}(i+1) \longrightarrow \text { Nuc }\left(d_{i}\right)(i+1) \longrightarrow 0
$$

é uma resolução linear para Nuc $\left(d_{i}\right)(i+1)$.

Passamos às definições de módulos e álgebras de Koszul.

Definição 3.2 (Módulo de Koszul) Seja $\Lambda$ uma álgebra graduada 0,1-split-básica e $M$ um $\Lambda$ módulo graduado. Dizemos que $M$ é um módulo de Koszul se $M$ possui uma resolução linear.

Do corolário acima, segue que todo módulo de Koszul é gerado em grau 0.

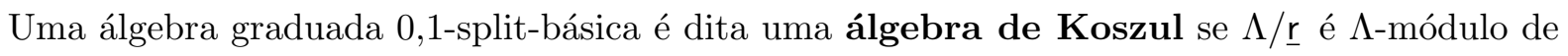
Koszul, ou equivalentemente, se cada $\Lambda$-módulo simples tem resolução linear.

\section{2 Álgebras de Koszul e suas álgebras de Yoneda}

A próxima proposição relaciona $\Lambda$-módulos que têm resoluções lineares de comprimento finito com certos módulos sobre a álgebra de extensões de $\Lambda$.

Proposição 3.4 ([18]) Seja $M$ um $\Lambda$-módulo gerado em grau 0, onde $\Lambda$ é uma álgebra graduada 0,1-split-básica. Se $M$ tem resolução linear de comprimento $n-1$, então $\operatorname{Ext}_{\Lambda}^{1}(\Lambda / \underline{r}, \Lambda / \underline{r})$. $\operatorname{Ext}_{\Lambda}^{j}(M, \Lambda / \underline{r})=\operatorname{Ext}_{\Lambda}^{j+1}(M, \Lambda / \underline{r})$, para todo $0 \leq j \leq n-1$.

Prova.

A prova será feita por indução sobre $n$. O caso $n=1$ será omitido porque ele é similar ao passo indutivo que segue. Assuma que o resultado é verdadeiro para todo $j=1, \ldots, k-1$. 
Seja $x \in \operatorname{Ext}_{\Lambda}^{k}(M, S)$ para algum módulo simples $S$ e considere uma resolução linear

$$
P_{k-1} \stackrel{d_{k-1}}{\longrightarrow} P_{k-2} \stackrel{d_{k-2}}{\longrightarrow} \cdots \longrightarrow P_{1} \stackrel{d_{1}}{\longrightarrow} P_{0} \stackrel{d_{0}}{\longrightarrow} M \longrightarrow 0
$$

de $M$ de comprimento $k-1$. Como $\operatorname{Ext}_{\Lambda}^{k}(M, S)$ é isomorfo a $\operatorname{Hom}_{\Lambda}\left(\operatorname{Nuc}\left(d_{k-1}\right), S\right)$, consideremos $g: H \rightarrow S\left(H:=\operatorname{Nuc}\left(d_{k-1}\right)\right)$ como a aplicação correspondente a $x$ sobre o isomorfismo acima. Dado

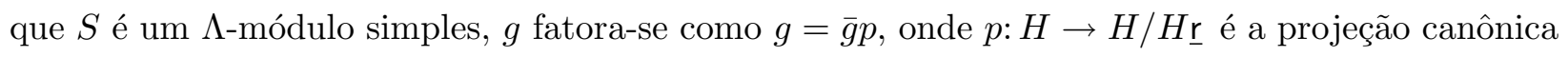
e $\bar{g}: H / H \underline{r} \rightarrow S$ é alguma aplicação. Pelo Lema 3.1 temos que $H \subseteq P_{k-1} \underline{\mathrm{r}}$ e $H \underline{\mathrm{r}}=P_{k-1} \underline{\mathrm{r}}^{2} \cap H$. Assim, temos o seguinte quadrado comutativo

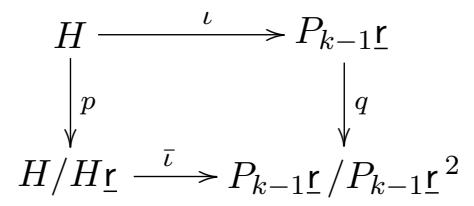

onde $\iota$ é a inclusão, $\iota$ é a aplicação induzida por $\iota$ e $q$ é a projeção canônica. Observe que $\bar{\iota}$ é injetora. Dado que $H / H \underline{r}$ e $P_{k-1} \underline{r} / P_{k-1} \underline{r}^{2}$ são $\Lambda$-módulos semisimples, então $\bar{\iota}$ é um monomorfismo que cinde. Assim, existe $p^{\prime}: P_{k-1} \underline{\mathrm{r}} \rightarrow H / H \underline{\mathrm{r}}$ tal que $p=p^{\prime} \iota$. Logo $g=\bar{g} p=\bar{g} p^{\prime} \iota$. Denotando $\bar{g} p^{\prime}: P_{k-1} \underline{\mathrm{r}} \rightarrow S$ por $g^{\prime}$, temos $g=g^{\prime} \iota$.

Agora considere o seguinte diagrama comutativo:

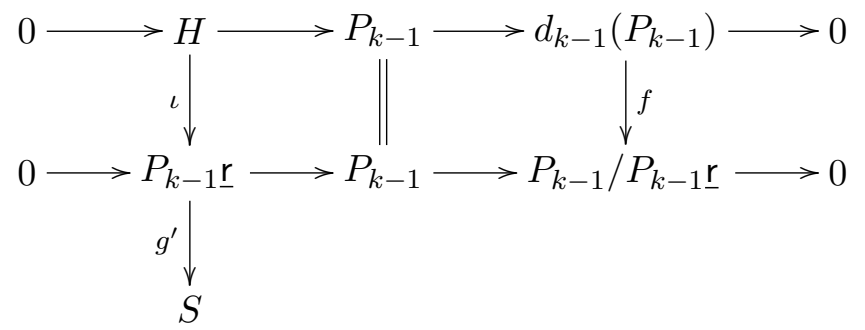

onde $f$ é a sobrejeção de $d_{k-1}\left(P_{k-1}\right)$ sobre seu topo. Seja $0 \rightarrow S \rightarrow E \rightarrow P_{k-1} / P_{k-1} \underline{r}$ o pushout de $S \stackrel{g^{\prime}}{\longleftarrow} P_{k-1} / \underline{r} P_{k-1} \longrightarrow P_{k-1}$. Suponhamos que $P_{k-1} / \underline{r} P_{k-1} \cong \bigoplus_{i=1}^{m} S_{i}$ onde cada $S_{i}$ é módulo simples. Agora $\operatorname{Hom}_{\Lambda}\left(f_{k-1}\left(P_{k-1}\right), \bigoplus_{i=1}^{m} S_{i}\right)$ é isomorfo a $\operatorname{Ext}_{\Lambda}^{k-1}\left(M, \bigoplus_{i=1}^{m} S_{i}\right)$. Assim, temos extensões $z_{i} \in \operatorname{Ext}_{\Lambda}^{k-1}\left(M, S_{i}\right)$ tais que $z=\left(z_{1}, \ldots, z_{m}\right)$ corresponde a $f \in \operatorname{Hom}_{\Lambda}\left(d_{k-1}\left(P_{k-1}\right), \bigoplus_{i=1}^{m} S_{i}\right)$. Seja $y=\left(y_{1}, \ldots, y_{m}\right)^{t}$ a extensão $0 \rightarrow S \rightarrow E \rightarrow \bigoplus_{i=1}^{m} S_{i} \operatorname{com} y_{i} \in \operatorname{Ext}_{\Lambda}^{1}\left(S_{i}, S\right)$. Então provamos que $x=z \cdot y=\sum_{i=1}^{m} z_{i} \cdot y_{i}$.

$\operatorname{Assim} \operatorname{Ext}_{\Lambda}^{k}(M, \Lambda / \underline{r})=\operatorname{Ext}_{\Lambda}^{1}(\Lambda / \underline{r}, \Lambda / \underline{r}) \cdot \operatorname{Ext}_{\Lambda}^{k-1}(M, \Lambda / \underline{r})$ e o resultado segue por indução.

O teorema seguinte nos diz que um $\Lambda$-módulo $M$ é Koszul se e somente se $E(M):=\bigoplus_{i \geq 0} \operatorname{Ext}_{\Lambda}^{i}(M, \Lambda / \underline{r})$ é gerado em grau 1 como um $E(\Lambda)$-módulo graduado.

Teorema 3.5 Sejam $\Lambda$ uma álgebra graduada 0,1-split-básica e $M$ um $\Lambda$-módulo graduado gerado em grau 0. Então as seguintes afirmações são equivalentes:

1. M é um módulo de Koszul. 
2. Para todo $j \geq 0$, tem-se que $\operatorname{Ext}_{\Lambda}^{j}(M, \Lambda / \underline{r}) \cdot \operatorname{Ext}_{\Lambda}^{1}(\Lambda / \underline{r}, \Lambda / \underline{r})=\operatorname{Ext}_{\Lambda}^{j+1}(M, \Lambda / \underline{r})$.

Prova.

A implicação "1. $\Rightarrow$ 2." segue da proposição anterior. Para a outra implicação, observemos inicialmente que como $\Lambda / \underline{r}=\bigoplus_{i=1}^{n} S_{i}$, então

$$
\begin{aligned}
\bigoplus_{i=1}^{n} \operatorname{Hom}(M, \Lambda / \underline{\mathbf{r}}) \cdot \operatorname{Ext}^{1}\left(\Lambda / \underline{r}, S_{i}\right) & =\operatorname{Hom}(M, \Lambda / \underline{r}) \cdot \operatorname{Ext}^{1}\left(\Lambda / \underline{r}, \oplus_{i=1}^{n} S_{i}\right) \\
& =\operatorname{Ext}^{1}\left(M, \oplus_{i=1}^{n} S_{i}\right) \\
& =\bigoplus_{i=1}^{n} \operatorname{Ext}^{1}\left(M, S_{i}\right) .
\end{aligned}
$$

Por outro lado, $\operatorname{como} \operatorname{Hom}(M, \Lambda / \underline{r}) \cdot \operatorname{Ext}^{1}\left(\Lambda / \underline{r}, S_{i}\right) \subseteq \operatorname{Ext}^{1}\left(M, S_{i}\right)$, segue da igualdade acima que

$$
\operatorname{Hom}(M, \Lambda / \underline{\mathrm{r}}) \cdot \operatorname{Ext}^{1}\left(\Lambda / \underline{\mathrm{r}}, S_{i}\right)=\operatorname{Ext}^{1}\left(M, S_{i}\right), \text { para todo } i
$$

Considere agora o início de uma resolução projetiva minimal graduada $P_{0} \rightarrow M \rightarrow 0$ de $M$. Como $M$ é gerado em grau 0 , então $P_{0}$ é gerado em grau zero. A implicação seguirá por indução, onde mostraremos que o núcleo de $P_{0} \rightarrow M$ é gerado em grau 1.

Considere a sequência exata $0 \rightarrow \Omega^{1} \rightarrow P_{0} \rightarrow M \rightarrow 0$ em gr $\Lambda$, onde $\Omega^{1}$ denota o núcleo da aplicação $P_{0} \rightarrow M$. Nestas condições, temos o seguinte diagrama comutativo com linhas exatas em $\operatorname{gr} \Lambda$ :

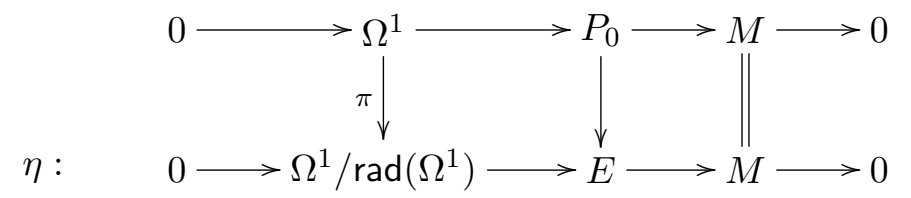

onde a linha superior é a sequência dada e a linha inferior é obtida através do pushout ${ }^{2} E$. Assim $\eta \in \operatorname{Ext}_{\Lambda}^{1}\left(M, \Omega^{1} / \operatorname{rad} \Omega^{1}\right)$.

Por outro lado, dado que $\Omega^{1} / \operatorname{rad}\left(\Omega^{1}\right)$ é um $\Lambda$-módulo semisimples, então da igualdade 3.1 temos que

$$
\operatorname{Hom}(M, \Lambda / \underline{r}) \cdot \operatorname{Ext}^{1}\left(\Lambda / \underline{r}, \Omega^{1} / \operatorname{rad} \Omega^{1}\right)=\operatorname{Ext}^{1}\left(M, \Omega^{1} / \operatorname{rad} \Omega^{1}\right)
$$

\footnotetext{
${ }^{2}$ Para a definição de pushout, veja [1] (pag. 432).
} 
e, com isso, $\eta=\sum f_{l} \xi_{l} \operatorname{com}$ cada $f_{l} \in \operatorname{Hom}(M, \Lambda / \underline{r})$ e cada $\xi_{l} \in \operatorname{Ext}^{1}\left(\Lambda / \underline{r}, \Omega^{1} / \operatorname{rad} \Omega^{1}\right)$. Pela definição de produto, temos o seguinte diagrama comutativo em $\operatorname{gr} \Lambda$ :

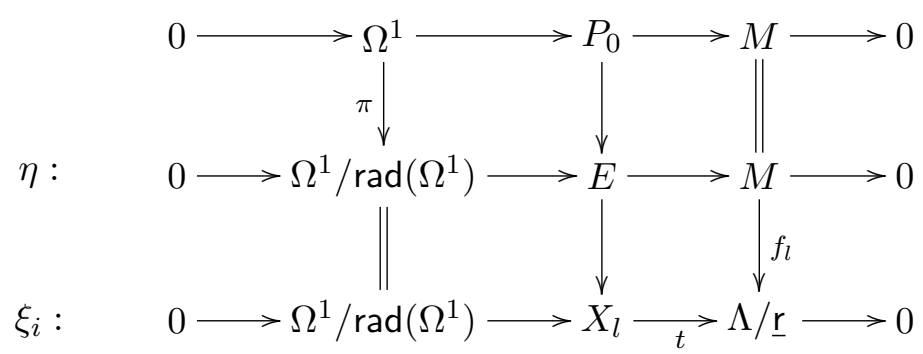

"Ocultando" a linha intermediária do diagrama acima, obtemos

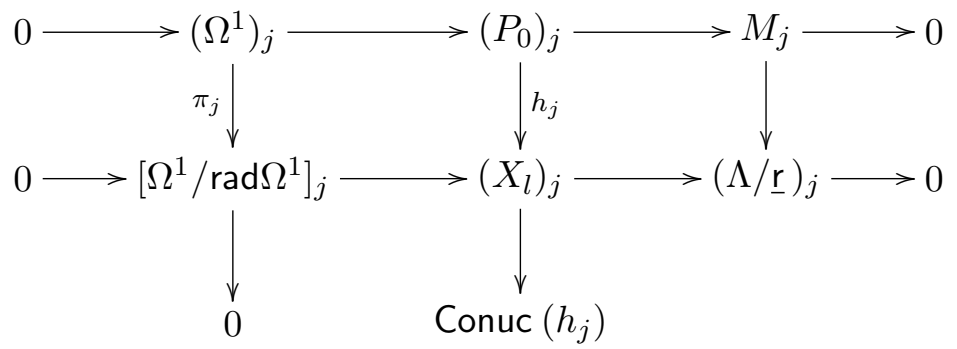

para todo $j \geq 0$. Assim, se tomarmos $j \geq 1$ e lembrarmos que $\Lambda / \underline{r}$ não tem nada em grau maior ou igual a 1 , teremos

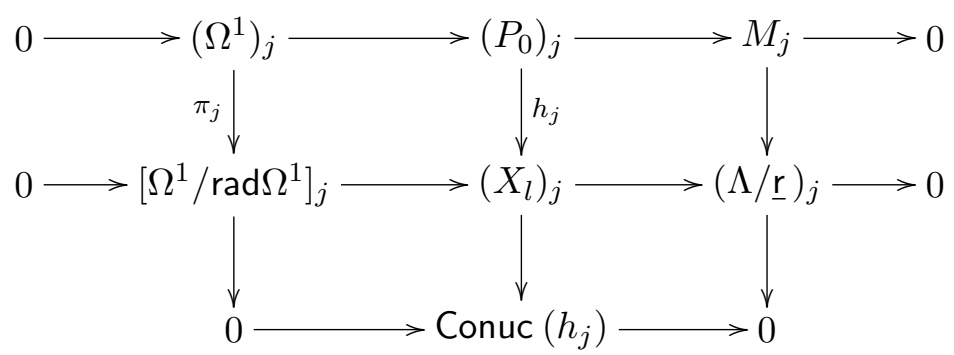

onde a linha inferior é exata. De onde segue que $h_{j}$ é um epimorfismo, para cada $j \geq 1$, e portanto qualquer elemento em $X_{l}$ de grau $j \geq 1$ é proveniente de um elemento em $P_{0}$ de grau $j \geq 1$. Neste caso, cada $X_{l}$ será gerado em grau 0 , pois assim é $P_{0}$.

Por outro lado, como $\Omega^{1} \subseteq \operatorname{rad} P_{0}$ e todos os elementos de $\operatorname{rad} P_{0}$ têm grau maior ou igual a 1 , então Nuc $(t)=\Omega^{1} / \operatorname{rad} \Omega^{1} \subseteq \bigoplus_{j \geq 1}\left(X_{l}\right)_{j} \subseteq$ Nuc $(t)$, onde a última inclusão é dada pelos fatos de $t$ preservar graus e $\Lambda / \underline{r}$ só ter elementos em grau 0 . Logo $\Omega^{1} / \operatorname{rad} \Omega^{1}=\bigoplus_{j \geq 1}\left(X_{l}\right)_{j}$ e como $X_{l}$ é gerado em grau 0 , segue que $\Omega^{1} / \operatorname{rad} \Omega^{1}$ é gerado em grau 1 .

Com o resultado do teorema acima temos uma caracterização para álgebras de Koszul.

Corolario 3.6 Sejam $\mathrm{k}$ um corpo, $\Gamma$ um carcás finito e $\Lambda=\mathrm{k} \Gamma / I$, onde $I$ é um ideal graduado contido em $J^{2}$. Então $\Lambda$ é uma álgebra de Koszul se e só se sua álgebra de extensões, E( $\left.\Lambda\right)$, é 
gerada em graus 0 e 1 .

Podemos agora enunciar um resultado importante que relaciona o carcás de uma álgebra de Koszul com o carcás de sua álgebra de Yoneda e para isso precisamos dos dois lemas que seguem.

Lema 3.7 Sejam $\Lambda$ uma k-álgebra e $M$ um $\Lambda$-módulo. Então

$$
\operatorname{Hom}_{\Lambda}(M, \Lambda / \underline{r}) \cong \operatorname{Hom}_{\Lambda / \underline{r}}(M / M \underline{r}, \Lambda / \underline{r})
$$

Prova.

Definamos $\phi: \operatorname{Hom}_{\Lambda}(M, \Lambda / \underline{r}) \rightarrow \operatorname{Hom}_{\Lambda / \underline{r}}(M / M \underline{r}, \Lambda / \underline{r})$ por $\phi(f)=\bar{f}$, onde $\bar{f}(m+M \underline{r}):=f(m)$. Não é difícil mostrar que $\phi$ é um isomorfismo de k-espaços vetoriais. Verificaremos apenas que $\phi$ é uma função.

Se $m-m^{\prime} \in M \underline{r}$, então existem $n_{i} \in M$ e $r_{i} \in \underline{r}$ tais que $m-m^{\prime}=\sum_{i} n_{i} r_{i}$. Logo

$$
f\left(m-m^{\prime}\right)=\sum_{i} f\left(n_{i}\right) r_{i}=\sum_{i}\left(\lambda_{i}+\underline{r}\right) r_{i}=0 \Rightarrow f(m)=f\left(m^{\prime}\right)
$$

Isto mostra que $\bar{f}$ é uma função. Além disso, é fácil verificar que $\bar{f}$ é um morfismo de $\Lambda / \underline{r}$-módulos e, portanto, $\phi$ é uma função.

Lema 3.8 Seja $R=\bigoplus_{i \geq 0} R_{i}$ uma k-álgebra graduada tal que $R_{1}=\underline{\mathrm{r}}_{R} / \underline{\mathrm{r}}_{R}^{2}$. Então $R$ é gerada em graus 0 e 1.

Prova.

Como $\underline{r}=0 \oplus R_{1} \oplus R_{2} \oplus \cdots$ é um ideal bilateral (graduado) de $R$, então

$$
\begin{aligned}
\underline{r}^{2}:=\underline{r} \cdot \underline{r} & =\left(0 \oplus R_{1} \oplus R_{2} \oplus \ldots\right) \cdot\left(0 \oplus R_{1} \oplus R_{2} \oplus \ldots\right) \\
& =0 \oplus 0 \oplus R_{1}^{2} \oplus\left(R_{1} R_{2}+R_{2} R_{1}\right) \oplus\left(R_{1} R_{3}+R_{2}^{2}+R_{3} R_{1}\right) \oplus \ldots
\end{aligned}
$$

o que implica que

$$
\frac{\underline{r}}{\underline{r}^{2}}=\frac{0}{0} \oplus \frac{R_{1}}{0} \oplus \frac{R_{2}}{R_{1}^{2}} \oplus \frac{R_{3}}{R_{1} R_{2}+R_{2} R_{1}} \oplus \frac{R_{4}}{R_{1} R_{3}+R_{2}^{2}+R_{3} R_{1}} \oplus \cdots
$$

Por hipótese $\underline{r} / \underline{r}^{2}=R_{1}$ e então da igualdade acima concluímos que $R_{2}=R_{1}^{2}, R_{3}=R_{1} R_{2}+$ $R_{2} R_{1}=R_{1} R_{1}^{2}+R_{1}^{2} R_{1}=R_{1}^{3}, R_{4}=R_{1} R_{3}+R_{2}^{2}+R_{3} R_{1}=R_{1} R_{1}^{3}+R_{1}^{2} R_{1}^{2}+R_{1}^{3} R_{1}=R_{1}^{4}, \ldots$ Em geral, $R_{n}=R_{1}^{n}$, para todo $n \geq 1$.

Proposição 3.9 Seja $\Lambda=\mathrm{k} \Gamma / I$ uma álgebra graduada 0,1-split-básica. Então $\Lambda$ é Koszul se, e somente se, $E(\Lambda)$ tem o mesmo carcás de $\Lambda$. 
Prova.

Suponhamos que $\Lambda$ é uma álgebra de Koszul. Dado que $\operatorname{Ext}_{\Lambda}^{0}(\Lambda / \underline{r}, \Lambda / \underline{r})=\operatorname{Hom}_{\Lambda}(\Lambda / \underline{r}, \Lambda / \underline{r}) \cong$ $(\Lambda / \underline{r})^{o p}$ é um anel semisimples, então $\underline{r}_{E}$ é o radical de Jacobson graduado de $E(\Lambda)$. Além disso, como $\Lambda$ é Koszul, então $E(\Lambda)$ é gerada em graus 0 e 1 e assim, da Proposição $1.12, E(\Lambda)=$ $\bigoplus_{n \geq 0} \underline{r}_{E}^{n} / \underline{r}_{E}^{n+1}$ como anéis graduados. Em particular, $\underline{r}_{E} / \underline{r}_{E}^{2} \cong \operatorname{Ext}_{\Lambda}^{1}(\Lambda / \underline{r}, \Lambda / \underline{r})$. Pela resolução de Butler $^{3}$

$$
0 \rightarrow I / I J \rightarrow J / I J \rightarrow \Lambda \stackrel{\pi}{\rightarrow} \Lambda / \underline{r} \rightarrow 0,
$$

obtemos o isomorfismo $\operatorname{Ext}_{\Lambda}^{1}(\Lambda / \underline{r}, \Lambda / \underline{r}) \cong \operatorname{Hom}_{\Lambda}(J / I, \Lambda / \underline{r})$, pois $J / I=\operatorname{Nuc}(\pi)$. Por outro lado, pelo Lema 3.7, temos que $\operatorname{Hom}_{\Lambda}(J / I, \Lambda / \underline{r}) \cong \operatorname{Hom}_{\Lambda / \underline{r}}\left(\underline{r} / \underline{r}^{2}, \Lambda / \underline{r}\right)$. Logo, se $e_{i}$ e $e_{j}$ são idempotentes em $E(\Lambda)_{0}$, então

$$
\begin{aligned}
& e_{i}\left(\underline{\mathrm{r}} E / \underline{\mathrm{r}}_{E}^{2}\right) e_{j} \cong e_{i} \operatorname{Ext}_{\Lambda}^{1}(\Lambda / \underline{\mathrm{r}}, \Lambda / \underline{\mathrm{r}}) e_{j} \cong e_{i} \operatorname{Hom}_{\Lambda}(J / I, \Lambda / \underline{\mathrm{r}}) e_{j} \cong e_{i} \operatorname{Hom}_{\Lambda / \underline{\mathrm{r}}}\left(\underline{\mathrm{r}} / \underline{\mathrm{r}}^{2}, \Lambda / \underline{\mathrm{r}}\right) e_{j} \\
& \cong \operatorname{Hom}_{\Lambda / \underline{\mathrm{r}}}\left(e_{i} \underline{\mathrm{r}} / e_{i} \underline{\underline{r}}^{2}, e_{j}(\Lambda / \underline{\mathrm{r}})\right) \cong \operatorname{Hom}_{\mathrm{k}}\left(e_{j}(\Lambda / \underline{\mathrm{r}}) \otimes_{\Lambda / \underline{\mathrm{r}}} e_{i} \underline{\mathrm{r}} / e_{i} \underline{\mathrm{r}}^{2}, \mathrm{k}\right) \cong D\left(e_{j}\left(\underline{\mathrm{r}} / \underline{\mathrm{r}}^{2}\right) e_{i}\right)
\end{aligned}
$$

onde $D:=\operatorname{Hom}_{\mathrm{k}}(-, \mathrm{k})$ é funtor dual e o penúltimo isomorfismo é dado pelo Teorema da Adjunção ${ }^{4}$.

Reciprocamente, observemos que se $\Lambda$ e $E(\Lambda)$ têm o mesmo carcás então para todos $i$ e $j$, $e_{i}\left(\underline{r}_{E} / \underline{r}_{E}^{2}\right) e_{j} \cong e_{i}\left(\underline{\mathrm{r}} / \underline{\mathrm{r}}^{2}\right) e_{j}$. Usando uma sequência de isomorfismos análoga à acima, obtemos $e_{i}\left(\underline{\mathbf{r}} E / \underline{r}_{E}^{2}\right) e_{j} \cong e_{i} \operatorname{Ext}_{\Lambda}^{1}(\Lambda / \underline{r}, \Lambda / \underline{r}) e_{j}$ e portanto $\underline{r}_{E} / \underline{r}_{E}^{2} \cong \operatorname{Ext}_{\Lambda}^{1}(\Lambda / \underline{r}, \Lambda / \underline{r})$. Pelo Lema 3.8 segue que $E(\Lambda)$ é gerada em graus 0 e 1 , e pelo Corolário $3.6 \Lambda$ é Koszul.

Proposição 3.10 Seja $\Lambda=\mathrm{k} \Gamma / I$ uma álgebra graduada 0,1-split-básica. Se $E(E(\Lambda))$ e $\Lambda$ são isomorfas como álgebras graduadas, então $\Lambda$ é uma álgebra de Koszul.

Prova.

Como uma álgebra graduada, $E(\Lambda)=E(\Lambda)_{0} \oplus E(\Lambda)_{1} \oplus E(\Lambda)_{2} \oplus \cdots$ onde $E(\Lambda)_{n}=\operatorname{Ext}_{\Lambda}^{n}\left(\Lambda_{0}, \Lambda_{0}\right)$, para todo $n \geq 0$. O radical graduado, $\underline{r}_{E}$, de $E(\Lambda)$ é $E(\Lambda)_{1} \oplus E(\Lambda)_{2} \oplus \cdots$. Pela prova do Lema 3.8 , vimos que

$$
\underline{r}_{E} / \underline{r}_{E}^{2} \cong E(\Lambda)_{1} \oplus X_{2} \oplus X_{3} \oplus \cdots
$$

Como k-espaços vetoriais vemos que $E(\Lambda)_{1} \subseteq \underline{r}_{E} / \underline{r}_{E}^{2}$ e $\operatorname{assim} \operatorname{dim}_{\mathrm{k}}\left(E(\Lambda)_{1}\right) \leq \operatorname{dim}_{\mathrm{k}}\left(\underline{\mathrm{r}} E / \underline{\mathrm{r}}_{E}^{2}\right)$. Além disso, temos também que $\operatorname{dim}_{k}\left(E(\Lambda)_{1}\right)=\operatorname{dim}_{k}\left(\Lambda_{1}\right)$.

Vamos mostrar que cada $X_{i}=0$. De fato, suponhamos que exista $i \geq 2$ tal que $X_{i} \neq 0$, então $\operatorname{dim}_{\mathbf{k}}\left(\underline{r}_{E} / \underline{r}_{E}^{2}\right)>\operatorname{dim}_{\mathbf{k}}\left(\Lambda_{1}\right)$. Por outro lado, temos a seguinte sequência de isomorfismos

$$
\begin{array}{r}
E(E(\Lambda))_{1}=\operatorname{Ext}_{E(\Lambda)}^{1}\left(E(\Lambda)_{0}, E(\Lambda)_{0}\right) \cong \operatorname{Hom}_{E(\Lambda)}\left(\underline{r}_{E}, E(\Lambda)_{0}\right) \cong \\
\cong \operatorname{Hom}_{E(\Lambda) / \underline{r}_{E}}\left(\underline{r}_{E} / \underline{r}_{E}^{2}, E(\Lambda)_{0}\right) \cong D\left(\underline{r}_{E} / \underline{r}_{E}^{2}\right) .
\end{array}
$$

\footnotetext{
${ }^{3}$ Veja [9].

${ }^{4}$ Se necessário veja o Exemplo 1.4 e o Teorema 1.8, respectivamente.
} 
Logo $\operatorname{dim}_{\mathrm{k}} E(E(\Lambda))_{1}=\operatorname{dim}_{\mathrm{k}} \underline{\mathrm{r}} E / \underline{\mathrm{r}}_{E}^{2}>\operatorname{dim}_{\mathrm{k}}\left(\Lambda_{1}\right)$, o que é um absurdo, uma vez que $E(E(\Lambda))$ e $\Lambda$ são isomorfas como álgebras graduadas.

Portanto, $\underline{r}_{E} / \underline{r}_{E}^{2} \cong E(\Lambda)_{1}$ e o resultado segue do Lema 3.8 e do Corolário 3.6.

Mostra-se em [19] a recíproca desta proposição, generalizando o item (c) do Teorema 2.3:

Teorema 3.11 Seja $\Lambda$ uma álgebra graduada 0,1-split-básica. Então $\Lambda$ é Koszul se, e somente se, $E(E(\Lambda))$ e $\Lambda$ são isomorfas como álgebras graduadas.

Segue desse resultado e do Corolário 3.6 que a álgebra de Yoneda de uma álgebra de Koszul é também Koszul.

\section{3 Álgebras de Koszul e álgebras quadráticas}

Nesta seção iremos estudar uma relação entre as álgebras de Koszul e seu conjunto de relações. Iniciamos relembrando o funtor Tor e com alguns lemas técnicos.

Sejam $\Sigma$ uma k-álgebra,$M_{\Sigma}$ um $\Sigma$-módulo à direita, ${ }_{\Sigma} N$ um $\Sigma$-módulo à esquerda e

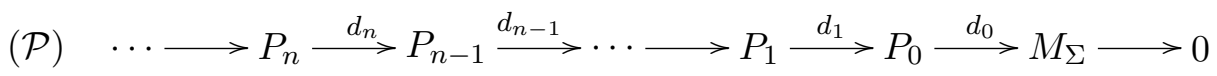

uma resolução projetiva de $M_{\Sigma}$. Aplicando o funtor $-\otimes_{\Sigma} N$ em $(\mathcal{P})$, obtemos um complexo

$$
\cdots \longrightarrow P_{n} \otimes_{\Sigma} N \stackrel{d_{n} \otimes 1}{\longrightarrow} P_{n-1} \otimes_{\Sigma} N \longrightarrow \cdots \longrightarrow P_{1} \otimes_{\Sigma} N \stackrel{d_{1} \otimes 1}{\longrightarrow} P_{0} \otimes_{\Sigma} N \longrightarrow 0 .
$$

Então

$$
\operatorname{Tor}_{n}^{\Sigma}(M, N)=\frac{\operatorname{Nuc}\left(d_{n} \otimes 1\right)}{\operatorname{Im}\left(d_{n+1} \otimes 1\right)}
$$

Lema 3.12 Sejam $\Lambda$ uma álgebra graduada 0,1-split-básica e

$$
\text { (P) } \quad \cdots \longrightarrow P_{n} \stackrel{d_{n}}{\longrightarrow} P_{n-1} \stackrel{d_{n-1}}{\longrightarrow} \cdots \longrightarrow P_{1} \stackrel{d_{1}}{\longrightarrow} P_{0} \stackrel{d_{0}}{\longrightarrow} \Lambda / \underline{\mathrm{r}} \longrightarrow 0
$$

uma resolução projetiva graduada minimal de $\Lambda / \underline{r}$. Então $\operatorname{Tor}_{n}^{\Lambda}(\Lambda / \underline{r}, \Lambda / \underline{r}) \cong P_{n} / \operatorname{rad} P_{n}$.

Lema 3.13 Seja $\Lambda=\mathrm{k} \Gamma / I$, onde $I \subseteq J^{2}$. Assim:

(a) Se $\Lambda$ é quadrática então $J^{3} \cap I=I J+J I$.

(b) Se $\Lambda$ é graduada e $J^{3} \cap I=I J+J I$ então $\Lambda$ é quadrática.

Prova.

(a) Como $I \subseteq J^{2}$, temos que $I J+J I \subseteq J^{3} \cap I$. Por outro lado, seja $x \in J^{3} \cap I$. Como $\Lambda$ é quadrátrica, temos que $x=\sum_{i=1}^{m} c_{i} \mu_{i} \rho_{i} \nu_{i}$, onde para cada $i=1, \ldots, m, \rho_{i}$ é uma relação de $\Lambda, \mu_{i}$ e $\nu_{i}$ são caminhos em $\Gamma$ e $c_{i} \in \mathrm{k} \backslash\{0\}$.

Afirmação: Nenhum dos $\mu_{i}$ e dos $\nu_{i}$, que aparecem na expressão acima, são vértices. 
$\triangleright$ De fato, se reescrevemos a expressão de $x$ na forma

$$
x=\sum_{\ell\left(\mu_{i}\right) \geq 1} c_{i} \mu_{i} \rho_{i} \nu_{i}+\sum_{j} c_{j} \rho_{j}^{\prime}
$$

teremos que cada termo $c_{i} \mu_{i} \rho_{i} \nu_{i}$ do primeiro somatório está em $J^{3}$ e, com isto,

$$
x-\sum_{\ell\left(\mu_{i}\right) \geq 1 \text { ou } \ell\left(\nu_{i}\right) \geq 1} c_{i} \mu_{i} \rho_{i} \nu_{i} \in J^{3} .
$$

Assim, como o comprimento de cada $\rho_{j}^{\prime}$ é dois, segue que $\sum_{j} c_{j} \rho_{j}^{\prime}=0$.

Da afirmação, temos que cada $\mu_{i}$ e cada $\nu_{i}$ ocorrendo na expressão $x=\sum_{i} c_{i} \mu_{i} \rho_{i} \nu_{i}$ tem comprimento maior ou igual a um e, portanto, $x \in I J+J I$. Isto conclui a prova da primeira parte.

(b) Assumamos que $\Lambda$ é graduada e seja $\rho=\sum_{j=1}^{t} c_{j} \gamma_{j} \in I \backslash(I J+J I)$ com todos os caminhos $\gamma_{j}$ de mesmo comprimento. Pela escolha de $\rho$ e da igualdade $I J+J I=J^{3} \cap I$ segue que $\rho \notin J^{3}$. Portanto o comprimento de cada $\gamma_{j}$ é dois e assim $I$ é quadrático.

Antes de mostrar dois resultados que relacionam álgebras quadráticas e álgebras de Koszul, é importante lembrar ao leitor uma fórmula de Butler ${ }^{5}$.

Teorema 3.14 ([9]) Sejam $\Lambda=\mathrm{k} \Gamma / I$, onde $I \subseteq J^{2}$. Então

$$
\operatorname{Tor}_{2 n}^{\Lambda}(\Lambda / \underline{r}, \Lambda / \underline{r}) \cong \frac{I^{n} \cap J I^{n-1} J}{J I^{n}+I^{n} J}, \text { para todo } n \geq 1
$$

$e$

$$
\operatorname{Tor}_{2 n+1}^{\Lambda}(\Lambda / \underline{\mathrm{r}}, \Lambda / \underline{\mathrm{r}}) \cong \frac{J I^{n} \cap I^{n} J}{I^{n+1}+J I^{n} J}, \text { para todo } n \geq 0
$$

O próximo resultado mostra que toda álgebra de Koszul é quadrárica.

Proposição 3.15 Seja $\Lambda=\mathrm{k} \Gamma / I$, onde $I \subseteq J^{2}$ é um ideal graduado. Se $\Lambda$ é uma álgebra de Koszul, então as relações de $\Lambda$ são combinações lineares de caminhos de comprimento 2 em $\Gamma$.

Prova.

Seja

$$
\cdots \rightarrow P_{n} \rightarrow \cdots \rightarrow P_{1} \rightarrow P_{0} \rightarrow \Lambda / \underline{r} \rightarrow 0
$$

uma resolução linear de $\Lambda / \underline{r}$ sobre $\Lambda$. Como a resolução acima é minimal, podemos identificar $P_{n} / \operatorname{rad} P_{n} \operatorname{com} \operatorname{Tor}_{n}^{\Lambda}(\Lambda / \underline{r}, \Lambda / \underline{r})$, para todo $n \geq 0$. Dado que $P_{2}$ é gerado em grau 2 e que

$$
\frac{I}{J I+I J}=\frac{I \cap J^{2}}{J I+I J}=\operatorname{Tor}_{2 \cdot 1}^{\Lambda}(\Lambda / \underline{r}, \Lambda / \underline{r})=\frac{P_{2}}{\operatorname{rad} P_{2}},
$$

\footnotetext{
${ }^{5}$ Veja [9].
} 
então $I /(J I+I J)$ é gerado por uma combinação linear de caminhos de comprimento 2 . Como $I$ é um ideal de $\mathrm{k} \Gamma$, temos que nenhum elemento $\rho$ de um sistema minimal de geradores de $I$ pertence a $I J+J I$. Dado que

$$
\rho+(J I+I J)=\sum_{i} \alpha_{i} p_{i}+(J I+I J) \neq 0
$$

onde cada $p_{i}$ tem grau 2, então $\rho-\sum_{i} \alpha_{i} p_{i} \in J I+I J \subseteq I$ e $\rho=\sum_{i} \alpha_{i} p_{i}+\left(\rho-\sum_{i} \alpha_{i} p_{i}\right)$. Pela minimalidade do sistema de geradores, $\rho=\sum_{i} \alpha_{i} p_{i}$.

Nem toda álgebra quadrática gerada em graus 0 e 1 é uma álgebra de Koszul. (Veja o próximo exemplo.) No entanto se a álgebra for quadrática monomial, então ela será Koszul, como mostrado no Teorema 2.3. Outra condição suficiente seria adicionarmos a hipótese de que a dimensão global de $\Lambda$ é dois, como mostra a próxima proposição.

Proposição 3.16 Seja $\Lambda$ uma álgebra quadrática, gerada em graus 0 e 1, de dimensão global igual a dois. Então $\Lambda$ é uma álgebra de Koszul.

Prova.

Considere uma resolução graduada minimal $0 \rightarrow P_{2} \rightarrow P_{1} \rightarrow P_{0} \rightarrow \Lambda / \underline{r} \rightarrow 0$ para o $\Lambda$-módulo $\Lambda / \underline{r}$. Como $\Lambda / \underline{r}$ é gerado em grau 0 , então $P_{0}$ é gerado em grau zero. Por outro lado, pela fórmula de Butler, segue que $P_{1} / \operatorname{rad} P_{1} \cong J /\left(I+J^{2}\right)=J / J^{2}$ e cada somando de $P_{1}$ é gerado em grau 1. Ainda pela fórmula de Butler, $P_{2} / \operatorname{rad} P_{2} \cong I /(I J+J I)$. Mas como $I$ é quadrático, então $J I+I J=\left(J^{3} \cap I\right)$ e assim $P_{2} / \operatorname{rad} P_{2}=I /\left(J^{3} \cap I\right)$, de onde segue que cada somando de $P_{2}$ é gerado em grau 2.

Exemplo 3.1 Sejam $\Gamma$ o carcás

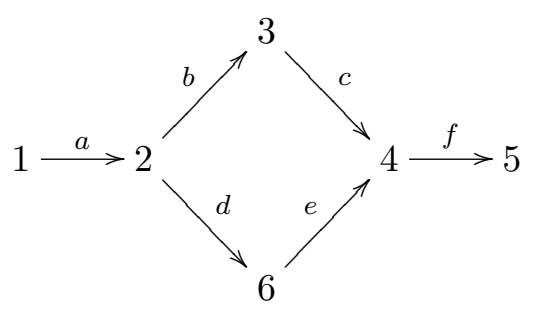

e I o ideal de $\mathrm{k} \Gamma$ dado por $I=\langle a b, e f, b c-d e\rangle$. Então $\Lambda=\mathrm{k} \Gamma / I$ é uma álgebra quadrática que não é Koszul. De fato, temos a seguinte resolução minimal para $S_{1}$ :

$$
0 \rightarrow P_{5}(-4) \stackrel{(c f)}{\longrightarrow} P_{3}(-2) \stackrel{(b)}{\longrightarrow} P_{2}(-1) \stackrel{(a)}{\longrightarrow} P_{1} \rightarrow S_{1} \rightarrow 0
$$

que não é linear, pois $(c f) v_{5}=c f \notin v_{3} \Lambda_{1}$ (veja Proposição 3.2). Observe que a dimensão global dessa álgebra é três. 


\section{4 Álgebra de Koszul vista como $\Lambda^{e}$-módulo}

Veremos agora uma outra caracterização para álgebras de Koszul e para esta caracterização precisamos de alguns resultados.

Sejam $\Lambda=\bigoplus_{n \geq 0} \Lambda_{n}$ e $\Sigma=\bigoplus_{n \geq 0} \Sigma_{n}$ k-álgebras graduadas. Então, como já observado antes,

$$
\Lambda \otimes_{\mathrm{k}} \Sigma=\bigoplus_{n \geq 0}\left(\sum_{i+j=n} \Lambda_{i} \otimes_{\mathrm{k}} \Sigma_{j}\right)
$$

é um álgebra graduda. Além disso, se $\Lambda$ e $\Sigma$ são 0,1 -split-básicas, então $\Lambda \otimes_{\mathrm{k}} \Sigma$ é também 0,1-split-básica.

Com a proposição a seguir, temos uma forma de construir álgebras de Koszul a partir do produto tensorial entre álgebras de Koszul.

Proposição 3.17 ([19]) Sejam $\Lambda$ e $\Sigma$ duas álgebras de Koszul. Então $\Lambda \otimes_{\mathrm{K}} \Sigma$ é uma álgebra de Koszul.

Exemplo 3.2 Sejam $\Gamma$ o carcás $\bigcirc x$ e $\Lambda=\mathrm{k} \Gamma$. Então $\Lambda$ é uma álgebra de Koszul. Além disso, $\Lambda \cong \mathrm{k}[x]$ e, pela proposição acima, temos que $\mathrm{k}\left[x_{1}, \ldots, x_{n}\right] \cong \mathrm{k}\left[x_{1}\right] \otimes_{\mathrm{k}} \cdots \otimes_{\mathrm{k}} \mathrm{k}\left[x_{n}\right]$ é uma álgebra de Koszul.

Para o que segue, precisaremos que a álgebra envolvente de uma álgebra de Koszul também seja uma álgebra Koszul. Para tanto, vamos assumir mais um resultado, o qual também pode ser encontrado em [19].

Proposição 3.18 ([19]) Se $\Lambda$ é uma álgebra de Koszul então sua álgebra oposta, $\Lambda^{o p}$, é uma álgebra de Koszul.

Como consequência dessas duas proposições temos que $\Lambda^{e}=\Lambda^{o p} \otimes_{\mathrm{k}} \Lambda$, a álgebra envolvente de $\Lambda$, é Koszul sempre que $\Lambda$ for uma álgebra de Koszul.

Antes de mostrar um resultado que nos fornece uma caracterização de álgebras de Koszul, primeiro mostraremos que se $\Lambda$ é uma álgebra de Koszul, então ela pode ser vista como um $\Lambda^{e}$ módulo graduado. No fundo, obteremos uma $\Lambda^{e}$-apresentação projetiva (minimal) para $\Lambda .^{6}$

Seja $\Lambda=k \Gamma / I$ uma álgebra graduada gerada em graus 0 e 1 . Como sempre, $\Gamma_{0}$ denotará os vértices de $\Gamma$ e $\Gamma_{1}$ o conjunto das flechas. Podemos ver $\Lambda$ como um $\Lambda^{e}$-módulo graduado da seguinte forma:

- Para cada vértice $v$ em $\Gamma$ sejam $\Lambda v$ e $v \Lambda$ os $\Lambda$-módulos graduados - à esquerda e à direita, respectivamente - projetivos indecomponíveis, gerados em grau 0. Então seja $P_{0}=$ $\bigoplus_{v \in \Gamma_{0}}\left(\Lambda v \otimes_{\mathrm{k}} v \Lambda\right)$. Como $P_{0}$ é um somando direto de $\Lambda^{e}$, segue que $P_{0}$ é um $\Lambda^{e}$-módulo projetivo. Além disso, como cada $\Lambda v \otimes_{\mathrm{k}} v \Lambda$ é um $\Lambda^{e}$-módulo graduado gerado em grau zero, então $P_{0}$ é um módulo projetivo graduado sobre $\Lambda^{e}$ gerado em grau 0.

\footnotetext{
${ }^{6}$ Para detalhes sobre essa construção, o leitor pode consultar Bardzell, [3,4]
} 
- Para cada flecha $a$ em $\Gamma$, denote por o $(a)$ e t $(a)$ a origem e o término de $a$, respectivamente. Nesta notação, considere $P_{1}=\bigoplus_{a \in \Gamma_{1}}\left(\Lambda \mathrm{o}(a) \otimes_{\mathrm{k}} \mathrm{t}(a) \Lambda\right)$. Olhando $\Lambda \mathrm{o}(a)$ como um $\Lambda$-módulo (projetivo) graduado gerado em grau 0 e $\mathrm{t}(a) \Lambda$ como um $\Lambda$-módulo (projetivo) gerado em grau 1, vemos que $P_{1}$ é um $\Lambda^{e}$-módulo graduado projetivo gerado em grau 1 . Rigorosamente, deveríamos ter escrito $P_{1}$ na forma $\bigoplus_{a \in \Gamma_{1}}\left(\Lambda \circ(a)[-1] \otimes_{\mathrm{k}} \mathrm{t}(a) \Lambda\right)$.

- Agora definimos o morfismo $\phi: P_{1} \rightarrow P_{0}$ pela fórmula

$$
\phi(\mathrm{o}(a) \otimes \mathrm{t}(a)):=a \otimes \mathrm{t}(a)-\mathrm{o}(a) \otimes a=a(\mathrm{o}(a) \otimes \mathrm{t}(a))-(\mathrm{o}(a) \otimes \mathrm{t}(a)) a .
$$

Então $\phi$ é um morfismo de $\Lambda^{e}$-módulos que preserva graus. Além disso, $\Lambda$ é o Conuc $(\phi)$ e, portanto, pode ser vista como $\Lambda^{e}$-módulo graduado.

Éinteressante chamar a atenção para o fato de que $P_{1} \rightarrow P_{0} \rightarrow \Lambda \rightarrow 0$ é uma apresentação projetiva minimal graduada de $\Lambda$ sobre $\Lambda^{e}$.

Agora estamos aptos a apresentar uma caracterização para álgebras de Koszul. Esta caracterização é devida a E. Green e R. Martinez-Villa. Porém, a prova que apresentaremos foi dada por E. N. Marcos.

Teorema 3.19 Seja $\Lambda$ uma k-álgebra graduada gerada em graus 0 e 1. Então $\Lambda$ é uma álgebra de Koszul se, e somente se, quando vista como um $\Lambda^{e}$-módulo possui uma resolução linear.

Prova.

Afirmação 1: Se $P=\Lambda v \otimes_{\mathrm{k}} w \Lambda$ é um $\Lambda^{e}$-módulo projetivo indecomponível e $M$ um $\Lambda$-módulo, então $M \otimes_{\Lambda} P \cong(w \Lambda)^{\operatorname{dim}(M v)}$.

$\triangleright$ De fato, se $V$ é um k-espaço vetorial com k-base $\left\{v_{t}\right\}_{t \in T}$, então

$$
V \otimes_{\mathrm{k}} w \Lambda \cong \bigoplus_{t \in T}\left(\mathrm{k} v_{t} \otimes_{\mathrm{k}} w \Lambda\right) \cong \bigoplus_{t \in T} v_{t}(w \Lambda) \cong(w \Lambda)^{(T)}
$$

Logo,

$$
M \otimes_{\Lambda} P \cong\left(M \otimes_{\Lambda} \Lambda v\right) \otimes_{\mathrm{k}} w \Lambda \cong M v \otimes_{\mathrm{k}} w \Lambda \cong(w \Lambda)^{\operatorname{dim}(M v)}
$$

Em particular, se $S$ é um $\Lambda$-módulo simples, então $S \otimes_{\Lambda} P \cong_{\Lambda} w \Lambda$ se $S \cong v \Lambda / v \underline{\text { r ou }} S \otimes_{\Lambda} P \cong 0$ caso contrário. Portanto, podemos escrever ainda $w \Lambda \cong \Lambda / \underline{r} \otimes_{\Lambda} P$. De onde segue que cada $\Lambda$-módulo projetivo $Q$ é isomorfo a $\Lambda / \underline{\mathrm{r}} \otimes_{\Lambda} P$, para algum $\Lambda^{e}$-módulo projetivo $P$.

Precisamos de mais um resultado para concluir nossa prova:

Afirmação 2: Seja $f: P \rightarrow Q$ um morfismo entre $\Lambda^{e}$-módulos projetivos finitamente gerados. Então $\operatorname{Im}(f) \subseteq Q \underline{r} \Lambda^{e}$ se, e somente se, $\operatorname{Im}\left(1_{S} \otimes_{\Lambda} f\right) \subseteq\left(S \otimes_{\Lambda} Q\right) \underline{r}$, para cada $\Lambda$-módulo simples $S$. $\triangleright$ Sem perda de generalidade, assumamos que $Q=\Lambda v \otimes w \Lambda$ é um $\Lambda^{e}$-módulo indecomponível.

$(\Leftarrow)$ Se $f$ não é sobrejetora, então $\operatorname{Im}(f)$ é um $\Lambda^{e}$-submódulo próprio de $Q$ e segue que $\operatorname{Im}(f) \subseteq$ $Q \underline{r}_{\Lambda}^{e}$, pois $Q \underline{r}_{\Lambda}{ }^{e}$ é o único submódulo maximal de $Q$. Por outro lado, se $f$ fosse sobrejetora, então 
a aplicação $1_{S} \otimes f: S \otimes_{\Lambda} P \rightarrow S \otimes_{\Lambda} Q$ também seria sobrejetora para todo $\Lambda$-módulo simples $S$. Escolhendo $S=v \Lambda / v \underline{r}$, teremos pela primeira afirmação que $S \otimes_{\Lambda} Q \cong w \Lambda$ (como $\Lambda$-módulos) e, portanto,

$$
w \Lambda \cong S \otimes_{\Lambda} Q=\operatorname{Im}(1 \otimes f) \subseteq\left(S \otimes_{\Lambda} Q\right) \underline{r} \cong w \underline{r} \subseteq w \Lambda
$$

ou seja, $w \underline{r}=w \Lambda$, que é uma contradição.

$(\Rightarrow)$ Primero observe que se $S$ é um $\Lambda$-módulo simples não isomorfo a $v \Lambda / v \underline{r}$, temos pela Afirmação 1 que $S \otimes_{\Lambda} Q=0$ e assim $0=\operatorname{Im}\left(1_{S} \otimes f\right)=\left(S \otimes_{\Lambda} Q\right) \underline{r} \Lambda^{e}$. Agora seja $S \cong v \Lambda / v \underline{r}$. Como $\underline{\mathrm{r}} \Lambda^{e}=\left(\underline{\mathrm{r}} \Lambda^{o p}\right) \otimes_{\mathrm{k}} \Lambda+\Lambda^{o p} \otimes_{\mathrm{k}} \underline{\mathrm{r}}$, temos que $Q \underline{\mathrm{r}}_{\Lambda^{e}}=\underline{\mathrm{r}} v \otimes_{\mathrm{k}} w \Lambda+\Lambda v \otimes_{\mathrm{k}} w \underline{\mathrm{r}}$. Por hipótese $\operatorname{Im}(f) \subseteq Q \underline{\mathrm{r}} \Lambda^{e}$, com isso

$$
\begin{aligned}
\operatorname{Im}\left(1_{S} \otimes f\right)=S \otimes \operatorname{Im}(f) & \subseteq S \otimes_{\Lambda}\left(\underline{r} v \otimes_{\mathrm{k}} w \Lambda\right)+S \otimes_{\Lambda}\left(\Lambda v \otimes_{\mathrm{k}} w \underline{r}\right) \\
& =0+S \otimes_{\Lambda}\left(\Lambda v \otimes_{\mathrm{k}} w \Lambda\right) \underline{r} \\
& =\left(S \otimes_{\Lambda} Q\right) \underline{r}
\end{aligned}
$$

Se $(\mathcal{P}): \quad \cdots \rightarrow P_{n} \rightarrow \cdots \rightarrow P_{0} \rightarrow \Lambda \rightarrow 0$ é uma resolução linear para $\Lambda_{\Lambda^{e}}$, então cada $P_{i}$ é gerado em grau $i$ e assim cada $\Lambda / \underline{r} \otimes_{\Lambda} P_{i}$ é gerado em grau $i$. Daí segue que

$$
\left(\mathcal{P} \otimes_{\Lambda} \Lambda / \underline{r}\right): \quad \cdots \rightarrow \Lambda / \underline{r} \otimes_{\Lambda} P_{n} \rightarrow \cdots \rightarrow \Lambda / \underline{r} \otimes_{\Lambda} P_{0} \rightarrow \Lambda / \underline{r} \rightarrow 0
$$

é uma resolução linear para $\Lambda / \underline{r}$.

Suponha agora que $(\mathcal{Q}): \quad \cdots \rightarrow Q_{n} \rightarrow \cdots \rightarrow Q_{0} \rightarrow \Lambda / \underline{r} \rightarrow 0$ é uma resolução linear para $\Lambda / \underline{r}$, que é portanto minimal. Seja

$$
(\mathcal{P}): \quad \cdots \rightarrow P_{n} \rightarrow \cdots \rightarrow P_{0} \rightarrow \Lambda \rightarrow 0
$$

uma resolução projetiva minimal para $\Lambda_{\Lambda^{e}}$. Pela segunda afirmação,

$$
\left(\mathcal{P} \otimes_{\Lambda} \Lambda / \underline{r}\right): \quad \cdots \rightarrow \Lambda / \underline{r} \otimes_{\Lambda} P_{n} \rightarrow \cdots \rightarrow \Lambda / \underline{r} \otimes_{\Lambda} P_{0} \rightarrow \Lambda / \underline{r} \rightarrow 0
$$

é uma resolução projetiva minimal para $\Lambda / \underline{\mathrm{r}}$ e, portanto, $Q_{i} \cong \Lambda / \underline{\mathrm{r}} \otimes_{\Lambda} P_{i}$, para todo $i$. Como cada $Q_{i}$ é gerado em grau $i$, então $P_{i}$ é gerado em grau $i$ e, portanto, $(\mathcal{P})$ é uma resolução linear. 


\section{Capítulo 4}

\section{Resoluções Projetivas}

O objetivo principal deste capítulo é apresentar um procedimento para a construção de resoluções projetivas de módulos sobre quocientes de álgebras de caminhos, isto é, álgebras da forma $\Lambda=\mathrm{k} \Gamma / I$, para algum ideal $I$ contido em $J^{2}$. Este procedimento é baseado em algumas propriedades dos elementos da resolução projetiva dada em [14] e, principalmente na teoria de Bases de Gröbner para álgebras de caminhos. Veremos o que podemos exigir do módulo em questão e do ideal $I$ para que este procedimento produza uma resolução projetiva onde cada termo é um $\Lambda$-módulo finitamente gerado. No final do capítulo mostraremos como modificar tal procedimento para a construção de uma resolução linear (minimal) de módulos lineares sobre álgebras de Koszul.

O desenvolvimento deste capítulo segue basicamente o que foi feito em $[13,14]$. Também, faremos uso de $[5,11,12]$.

\subsection{Introdução}

Na maior parte desta seção, $\Gamma$ será um carcás finito, k um corpo, $R$ uma k-álgebra e $\mathcal{B}$ uma k-base para $R$. Faz-se necessário as seguintes convenções:

- se $\mathcal{I}$ é um conjunto de índices e $r_{i} \in R$ para cada $i \in \mathcal{I}$, então $\sum_{i \in \mathcal{I}} r_{i}$ denota uma soma onde quase todos os $r_{i}$ 's são nulos, isto é, existe apenas um número finito de índices $i$ para os quais $r_{i}$ é não nulo. Por outro lado, se escrevemos $\sum_{i \in \mathcal{I}} \alpha_{i} b_{i} \operatorname{com} \alpha_{i} \in \mathrm{k}$ e $b_{i} \in \mathcal{B}$, então quase todos os escalares $\alpha_{i}$ 's são iguais a zero e, a menos de mensão em contrário, todos os $b_{i}$ 's são distintos.

- Seja $r \in R$ tal que $r=\sum_{i \in \mathcal{I}} \alpha_{i} b_{i}$ com $\alpha_{i} \in \mathrm{k}$ e $b_{i} \in \mathcal{B}$. Diremos que $b_{j}$ ocorre em $r$ se $\alpha_{j} \neq 0$. Alguns autores dizem, neste caso, que $b_{j}$ é um elemento do suporte de $r$.

Sejam $\mathcal{B}=\left\{b_{i}\right\}_{i \in \mathcal{I}}$ uma k-base para $R,>$ uma boa ordem sobre $\mathcal{B}$ e $x=\sum_{i \in \mathcal{I}} \alpha_{i} b_{i}$ um elemento não nulo de $R$, com cada $\alpha_{i} \in \mathrm{k}$ e os $b_{i}$ 's em $\mathcal{B}$. O termo líder de $x$, denotado por tip $(x)$, é o maior elemento $b_{i}$ (com respeito a ordem $>$ ) que ocorre na expressão de $x$ acima, isto é, tip $(x)=b_{i}$ se e só se $b_{i}$ ocorre em $x$ e $b_{i}>b_{j}$ para todo $b_{j} \neq b_{i}$, com $b_{j}$ ocorrendo em $x$. O escalar $\alpha_{i}$ que aparece na decomposição $x=\sum_{i=1}^{n} \alpha_{i} b_{i}$ "acompanhando" o $b_{i}=\operatorname{tip}(x)$ é chamado de coeficiente líder de $x$ e denotado por $\mathrm{c} \ell(x)$. Quando $X$ é um subconjunto de $R$, definimos tip $(X)$ como sendo o subconjunto de $\mathcal{B}$ formado pelos termos líderes dos elementos não nulos de $X$, ou seja, 
$\operatorname{tip}(X):=\{b \in \mathcal{B}:$ existe $x \in X \backslash\{0\}$ de modo que $b=\operatorname{tip}(x)\}$. Note que $\operatorname{tip}(X)$ e tip $(x)$ dependem tanto da base $\mathcal{B}$ quanto da escolha da boa ordem $>$ sobre $\mathcal{B}$.

\section{Bases de Gröbner}

No que segue, faremos uma pequena exposição sobre a teoria de bases de Gröbner para álgebras associativas. Como referência indicamos $[5,11,12]$. Para tanto, precisamos definir os conceitos de base multiplicativa e de ordem admissível.

Definição 4.1 Seja $R$ uma k-álgebra. Dizemos que $\mathcal{B}$ é uma base multiplicativa para $R$ se $\mathcal{B}$ é uma k-base de $R$ e $b_{1} \cdot b_{2} \in \mathcal{B} \cup\{0\}$, para todos $b_{1}, b_{2} \in \mathcal{B}$.

É interessante destacar que esta noção de base multiplicativa foi definida em [6], porém a definição encontrada em [6] é mais restritiva que a que exigimos aqui.

Exemplo 4.1 Se $R=\mathrm{k} \Gamma$ e $\mathcal{B}$ é a $\mathrm{k}$-base usual de $R$, isto é, o conjunto de todos caminhos em $\Gamma$, então $\mathcal{B}$ é uma base multiplicativa para $R$.

Agora seja $R$ uma k-álgebra com base multiplicativa $\mathcal{B}$. Para o que queremos a seguir, apenas uma boa ordem arbitrária sobre $\mathcal{B}$ não é suficiente, precisamos de uma boa ordem que se comporte bem com a estrutura multiplicativa da base $\mathcal{B}$. Para tanto, indroduziremos o conceito de ordem admissível.

Definição 4.2 Seja $R$ uma k-álgebra com uma base multiplicativa $\mathcal{B}$. Dizemos que uma boa ordem $>($ sobre $\mathcal{B})$ é uma ordem admissível para $\mathcal{B}$ se as seguintes propriedades são verdadeiras:

- Para todos $b_{1}, b_{2}, b_{3} \in \mathcal{B}$, se $b_{1}>b_{2}$ então $b_{1} b_{3}>b_{2} b_{3}$, sempre que $b_{1} b_{3}$ e $b_{2} b_{3}$ forem não nulos.

- Para todos $b_{1}, b_{2}, b_{3} \in \mathcal{B}$, se $b_{1}>b_{2}$ então $b_{3} b_{1}>b_{3} b_{2}$, sempre que $b_{3} b_{1}$ e $b_{3} b_{2}$ forem não nulos.

- Para todos $b_{1}, b_{2}, b_{3}, b_{4} \in \mathcal{B}$, se $b_{1}=b_{2} b_{3} b_{4}$, então $b_{1} \geq b_{3}$.

A título de ilustração, faremos um exemplo que exibe uma ordem admissível sobre a base usual de $k \Gamma$. Há diversas outras ordens admissíveis sobre a álgebra de caminhos, o leitor pode encontrar uma boa variedade destas em, por exemplo, $[5,11]$.

Exemplo 4.2 (Grau-Lexicográfica) Seja $R=\mathrm{k} \Gamma$ a álgebra de caminhos do carcás $\Gamma$. Ordene totalmente e arbitrariamente os vértices e as flechas de $\Gamma$ de modo que cada vértice seja menor que qualquer flecha. Se p e q são caminhos de comprimento maior ou igual a um, então $p>q$ se o comprimento de p é maior que o comprimento de $q$, ou se os comprimentos são iguais e $p=p_{1} \ldots p_{n}$ e $q=q_{1} \ldots q_{n}$, onde os $p_{i}$ 's e os $q_{i}$ 's são flechas, então existe algum $i$ tal que $p_{j}=q_{j}$ se $j<i e$ $p_{i}>q_{i}$. Assim definida, $>$ é uma ordem admissivel sobre o conjunto de todos os em $\Gamma$. Essa ordem é chamada grau-lexicográfica. 
Usaremos a terminologia " $R$ possui uma base multiplicativa ordenada $(\mathcal{B},>)$ " para indicar que $\mathcal{B}$ é uma base multiplicativa para $R$ e $>$ é uma ordem admissível sobre $\mathcal{B}$.

É interessante mencionar que o uso de ordens admissíveis aparece frequentemente em problemas computacionais. Escolher uma ordem adequada é uma arte. Um problema pode não ter solução algorítmica com uma determinada ordem e ter com outra.

Definiremos agora um conceito que será útil para o desenvolvimento do texto.

Definição 4.3 Sejam $R$ uma k-álgebra com base multiplicativa ordenada $(\mathcal{B},>)$ e I um ideal de $R$. Dizemos que um subconjunto $\mathcal{G}$ de I é uma base de Gröbner para $I$, com respeito a $(\mathcal{B},>)$, se

$$
\langle\operatorname{tip}(\mathcal{G})\rangle=\langle\operatorname{tip}(I)\rangle
$$

isto é, o ideal gerado pelos termos líderes de $\mathcal{G}$ é igual ao gerado pelos termos líderes de $I$.

Observe que esta definição é equivalente a dizer o seguinte:

- dado $x \in I$, então existem $g \in \mathcal{G}$ e $p, q \in \mathcal{B}$ tais que $\operatorname{tip}(x)=p \operatorname{tip}(g) q$, isto é, $\operatorname{tip}(g) \mid \operatorname{tip}(x)$.

Sempre existe base de Gröbner para um dado ideal $I$, uma vez que o próprio $I$ é uma base de Gröbner. Em ([11], §2.4), por exemplo, encontramos um procedimento que constrói uma base de Gröbner para um ideal $I=\left\langle f_{1}, \ldots, f_{n}\right\rangle$ gerado por um conjunto finito de elementos uniformes em $R$. O procedimento produz uma sequência (possivelmente infinita) de elementos uniformes $g_{1}, g_{2}, \ldots$, onde $g_{i}=f_{i}$ para $1 \leq i \leq n$ e, para $i>n, g_{i} \in I$ é tal que

$$
\operatorname{tip}\left(g_{i}\right) \notin\left\langle\operatorname{tip}\left(g_{1}\right), \operatorname{tip}\left(g_{2}\right), \ldots, \operatorname{tip}\left(g_{i-1}\right)\right\rangle,
$$

de forma que o conjunto $\mathcal{G}=\left\{g_{1}, g_{2}, \ldots, g_{n}, g_{n+1}, \ldots\right\}$ é uma base de Gröbner para $I$. Além disso, quando o ideal gerado pelo tip $(I)$ possui um conjunto finito de (monômios) geradores, então este procedimento é algorítmico, isto é, ele produz uma base de Gröbner num número finito de passos.

Usamos tal procedimento para encontrar bases de Gröbner no exemplo a seguir.

Exemplo 4.3 Sejam $\Gamma$ o carcás

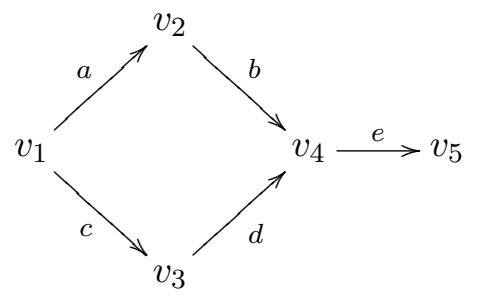

e I o ideal de $\mathrm{k} \Gamma$ gerado pelo conjunto $\{a b-c d, b e\}$.

(a) Se Escolhemos $>_{1}$ como a ordem grau-lexicográfica induzida por $a>b>c>d>e>v_{1}>$ $v_{2}>v_{3}>v_{4}>v_{5}$, então o conjunto $\mathcal{G}=\{a b-c d, b e, c d e\}$ é uma base de Gröbner para o 
ideal I. Além disso, qualquer subconjunto próprio de $\mathcal{G}$ não é base Gröbner (com respeito a ordem $\left.>_{1}\right)$. Em particular, o conjunto gerador $\{a b-c d, b e\}$ de I não é base de Gröbner.

(b) Se escolhemos $>_{2}$ como a ordem grau-lexicográfica induzida por $e>d>\ldots>a>v_{1}>v_{2}>$ $\ldots>v_{5}$, então $\mathcal{G}=\{a b-c d, b e\}$ é base de Gröbner.

Existem alguns tipos de bases de Gröbner que são, em certo sentido, melhores que as demais. Dentre estas, precisamos das bases de Gröbner denominadas reduzida e tip-reduzida. Mas antes de definir o que é uma base de Gröber reduzida, precisamos do seguinte resultado.

Lema 4.1 ([11]) Sejam $R$ uma k-álgebra com base multiplicativa ordenada $(\mathcal{B},>)$. Se I é um ideal monomial de $R$ então I possui um único conjunto gerador monomial minimal. Além disso, todo conjunto gerador monomial o contém.

Nestas condições, se $\mathcal{G}$ é uma base de Gröbner de $I$, então tip $(\mathcal{G})$ deve conter o conjunto minimal que gera o ideal monomial $\langle\operatorname{tip}(I)\rangle$.

Definição 4.4 (Base de Gröbner reduzida) Sejam $R$ uma k-álgebra com base multiplicativa ordenada $(\mathcal{B},>)$ e I um ideal de $R$. Uma base de Gröbner $\mathcal{G}$ de I satisfazendo as três condições

1. $c \ell(g)=1$, para todo $g \in \mathcal{G}$

2. o conjunto tip $(\mathcal{G})$ é o conjunto gerador monomial minimal de $\langle\operatorname{tip}(I)\rangle$

3. todo elemento $g \in \mathcal{G}$ é reduzido, isto é, qualquer elemento do conjunto $\mathcal{B} \backslash\{\operatorname{tip}(g)\}$ que ocorre em g pertence a $\mathcal{B} \backslash \operatorname{tip}(\mathcal{G})$

é dita base de Gröbner reduzida do ideal $I$.

O resultado seguinte, que será utilizado livremente no texto, garante a existência de uma única base de Gröbner reduzida para um ideal $I$ de uma k-álgebra com base multiplicativa ordenada.

Teorema 4.2 ([11]) Sejam $R$ uma k-álgebra com base multiplicativa ordenada $(\mathcal{B},>)$ e I um ideal de $R$. Então I possui uma única base de Gröbner reduzida (com respeito a ordem >).

É interessante observar que a propriedade 3 da Definição 4.4 é essencial para a unicidade da base de Gröbner reduzida do teorema acima. (Veja, por exemplo, [5].)

Uma outra classe de bases de Gröbner, contendo as bases de Gröbner reduzida, são as tipreduzidas.

Definição 4.5 Seja $X$ um subconjunto de $R$. Dizemos que $X$ é tip-reduzido se não existem elementos distintos $x$ e $y$ em $X$ tais que $\operatorname{tip}(x) \mid \operatorname{tip}(y)$. 
Assim, um conjunto $\mathcal{G}$ é dito uma base de Gröbner tip-reduzida se $\mathcal{G}$ é uma base de Gröbner que é um conjunto tip-reduzido.

Lembremos a definição de uma classe de elementos da álgebra de caminhos $\mathrm{k} \Gamma$, a qual será bastante utilizada no desenrolar do texto.

Definição 4.6 Dizemos que um elemento não nulo $x$ na álgebra de caminhos $R=\mathrm{k} \Gamma$ é uniforme à direita (à esquerda) se existe um vértice $v$ em $\Gamma$ tal que $x v=x(v x=x)$. Se existem vértices $v$ e $w$ tais que $v x w=x$, então $x$ é dito uniforme. ${ }^{1}$

De maneira análoga, se $M$ é um kГ-módulo e $m \in M$ é tal que $m v=m$, para algum $v \in \Gamma_{0}$, então $m$ é dito uniforme á direita.

\section{Bases de Gröbner à direita}

Existem também as noções de base de Gröbner (à direita) e ordem admissível para módulos sobre k-álgebras com uma base multiplicativa ordenada. E isto é o que passaremos a apresentar. Todos os resultados expostos aqui podem ser encontrado em [11].

Até o final desta seção, a menos de menção em contrário, $R$ denotará uma k-álgebra com base multiplicativa ordenada $(\mathcal{B},>)$ e $M$ um $R$-módulo à direita. Assim como em álgebras, precisamos de uma k-base para $M$ e de uma ordem admissível sobre ela.

Seja $\mathcal{M}$ uma k-base de $M$. Dizemos que $\mathcal{M}$ é uma base coerente (com respeito a $\mathcal{B}$ ) se para todo $m \in \mathcal{M}$ e todo $b \in \mathcal{B}, m b=0$ ou $m b \in \mathcal{M}$. (Observe que todo elemento de uma base coerente é uniforme à direita.) Por outro lado, dizemos que uma boa ordem $\succ$ sobre uma base coerente $\mathcal{M}$ é uma ordem admissível à direita sobre $\mathcal{M}$ se as seguintes condições são satisfeitas:

- para todos $m_{1}, m_{2} \in \mathcal{M}$ e todo $b \in \mathcal{B}$, se $m_{1} \succ m_{2}$ então $m_{1} b \succ m_{2} b$, sempre que $m_{1} b$ e $m_{2} b$ forem não nulos.

- para todo $m \in \mathcal{M}$ e todos $b_{1}, b_{2} \in \mathcal{B}$, se $b_{1}>b_{2}$ então $m b_{1} \succ m b_{2}$, sempre que $m b_{1}$ e $m b_{2}$ forem não nulos.

Como anteriormente, a título de simplificação, escrevemos " $(\mathcal{M}, \succ)$ é uma base ordenada de $M$ " para dizer que $\mathcal{M}$ é uma k-base coerente e $\succ$ é uma ordem admissível à direita sobre $\mathcal{M}$.

Exemplo 4.4 Sejam $R$ a álgebra de caminhos de um carcás $\Gamma$ e $M$ o $R$-módulo à direita vR,

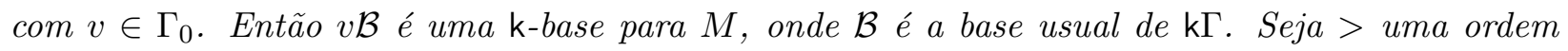
admissivel sobre $\mathcal{B}$. Definindo $\succ$ como a ordem $>$ restrita ao conjunto vB temos que $(v \mathcal{B}, \succ)$ é uma base ordenada de $M$.

Na próxima seção mostraremos que somas (diretas) arbitrárias destes tipos de $R$-módulos "sempre" admite bases ordenadas.

\footnotetext{
${ }^{1}$ Esta definição pode ser estendida para uma k-álgebra com base multiplicativa ordenada. Veja, por exemplo, seção $\S 3$ de [12].
} 
Definição 4.7 Seja $\left(\mathcal{M}=\left\{m_{i}\right\}_{i \in \mathcal{I}}, \succ\right)$ uma base ordenada de $M$.

(a) Se $m=\sum_{i \in \mathcal{I}} \alpha_{i} m_{i}$ é um elemento não nulo de $M$ (com cada $\alpha_{i} \in \mathrm{k}$ ) então o termo líder de $m$, denotado por tip $(m)$, é o maior elemento $m_{i}$ (com respeito a ordem $\succ$ ) que ocorre na decomposição de $m$.

(b) Se $X$ é um subconjunto de $M$ então $\operatorname{tip}(X):=\left\{m_{i} \in \mathcal{M}: m_{i}=\operatorname{tip}(x)\right.$ para algum $x \in$ $X \backslash\{0\}\}$.

(c) Sejam $N$ um submódulo de $M$ e $\mathcal{G}$ um subconjunto de $N$. Dizemos que $\mathcal{G}$ é uma base de Gröbner à direita de $N$ se o submódulo de $M$ gerado por tip $(\mathcal{G})$ é igual ao submódulo de $M$ gerado por tip $(N)$. Se além disso, para quaisquer $g, g^{\prime} \in \mathcal{G}$, distintos, não existir $b \in \mathcal{B}$ tal que $\operatorname{tip}\left(g^{\prime}\right)=\operatorname{tip}(g) b$, dizemos que $\mathcal{G}$ é tip-reduzida.

Observação 4.1 Se $\mathcal{G}$ é uma base de Gröbner à direita para um submódulo $N$ de um módulo com base ordenada, então $\mathcal{G}$ gera $N$ como um submódulo. ${ }^{2}$

Assim como na última parte do item (c) da definição acima, se $m$ e $m^{\prime}$ são elementos de $\mathcal{M}$ tais que existe $b \in \mathcal{B}$ de forma que $m^{\prime}=m b$, dizemos que $m$ divide $m^{\prime}$ à esquerda e denotamos por $\left.m\right|_{e} m^{\prime}$.

O próximo lema, que será utilizado livremente no decorrer do texto, mostra que é sempre possível encontrar uma base de Gröbner à direita tip-reduzida para um submódulo de um módulo com base de Gröbner ordenada. A prova que exibimos usa argumentos similares aos que serão utilizados na Proposição 4.9.

Lema 4.3 Sejam $R$ uma k-álgebra com base multiplicativa ordenada $(\mathcal{B},>)$ e $M$ um $R$-módulo com base ordenada $(\mathcal{M}, \succ)$. Se $N$ é um submódulo de $M$, então existe uma base de Gröbner à direita tip-reduzida com respeito $a \succ$.

Prova.

Sejam $T:=\operatorname{tip}(N)$ e $T^{*}=\left\{t \in T\right.$ : se existe $t^{\prime} \in T$ tal que $\left.t^{\prime}\right|_{e} t$, então $\left.t^{\prime}=t\right\}$.

Afirmação: $T^{*}$ é diferente do conjunto vazio.

$\triangleright$ De fato, se $t_{0} \in T$ e existe $t_{1} \in T \backslash\left\{t_{0}\right\}$ tal que $\left.t_{1}\right|_{e} t_{0}$, então $t_{0} \succ t_{1}$. Continuando este processo, produzimos uma cadeia da forma

$$
t_{0} \succ t_{1} \succ \cdots \succ t_{n} \succ \cdots
$$

onde cada $t_{i}$, para $i>0$, pertence ao conjunto $T \backslash\left\{t_{0}, \ldots, t_{i-1}\right\}$. Por outro lado, como $\succ$ é uma boa ordem, então existe algum $m$ tal que $t_{m}=t_{m+1} \cdot \triangleleft$

Para cada $t \in T^{*}$, seja $n_{t} \in N$ tal que tip $\left(n_{t}\right)=t$ e considere o conjunto $N^{*}=\left\{n_{t}: t \in T^{*}\right\} \subseteq N$. Afirmamos que $N^{*}$ é uma base de Gröbner à direita tip-reduzida para o submódulo $N$. De fato,

\footnotetext{
${ }^{2}$ Veja, por exemplo, [12] - Proposição 4.2.
} 
basta verificar que é uma base de Gröbner à direita, pois que $N^{*}$ é tip-reduzido segue da definição de $T^{*}$. Além disso, é claro que o $R$-submódulo de $M$ gerado por tip $\left(N^{*}\right)=T^{*}$ está contido no submódulo gerado por tip $(N)$. Por outro lado, se $t \in T \backslash T^{*}$ então existe $t^{\prime} \in T$ tal que $\left.t^{\prime}\right|_{e} t$. Dado que $\succ$ é uma boa ordem e $t b \succ t$, para todo $b \in \mathcal{B}$ tal que $t b \neq 0$, segue que existem $t^{*} \in T^{*}$ e $b \in \mathcal{B}$ de modo que $t=t^{*} b$. Isto mostra que $t$ pertence ao submódulo de $M$ gerado por $\operatorname{tip}\left(N^{*}\right)$.

Observação 4.2 No lema acima, se $R=\mathrm{k} \Gamma$ e $\mathcal{B}$ é a base usual de $R$, então $T^{*} \subseteq \mathcal{B}$ é um conjunto enumerável. Isto mostra que base de Gröbner à direita $N^{*}:=\left\{n_{t}: t \in T^{*}\right\}$, obtida na demonstração, é enumerável.

Antes de prosseguir com outros resultados, faremos mais uma observação sobre o último acima. Para tanto, recordamos que se $\Gamma$ é um carcás finito, então a unidade de $R=\mathrm{k} \Gamma$ é dada pela soma $\sum_{v \in \Gamma_{0}} v$. Logo, se $x$ é um elemento de $R$ (de $M$ ) então $x=\sum_{v \in \Gamma_{0}} x v$, ou seja, qualquer elemento não nulo $x$ em $R$ (em $M$ ) pode ser escrito como uma soma de elementos uniformes à direita. Portanto, com as hipóteses do Lema 4.3, se $\mathcal{G}^{\prime}$ é uma base de Gröbner à direita tip-reduzida para um submódulo $N$ de um k $\Gamma$-módulo $M$, então o conjunto $\mathcal{G}=\left\{g v: g \in \mathcal{G}^{\prime}, v \in \Gamma_{0}\right.$ e tip $\left.(g v)=\operatorname{tip}(g)\right\}$ é uma base de Gröbner à direita tip-reduzida uniforme para $N$. De fato, dado que tip $(\mathcal{G}) \subseteq \mathcal{M}$ e $\mathcal{M}$ é uma k-base coerente para $M$, então para cada $g \in \mathcal{G}$ existe único $v \in \Gamma_{0}$ tal que $\operatorname{tip}(g) v=\operatorname{tip}(g)$.

Antes de concluir este pequeno resumo sobre bases de Gröbner à direita, mostraremos um "exemplo" de uma base de Gröbner à direita tip-reduzida.

Exemplo 4.5 (Teorema 7.1 de [12]) Sejam $R=\mathrm{k} \Gamma, \mathcal{B}$ sua base usual com uma ordem admissivel $>$ e $I$ um ideal de $R$ com uma base de Gröbner $\mathcal{G}$ tip-reduzida consistindo de elementos uniformes. Então o conjunto

$\mathrm{rt} \mathcal{G}=\left\{p g: p \notin \operatorname{tip}(I), g \in \mathcal{G}\right.$, se existem $r, s \in \mathcal{B}$ e $g^{\prime} \in \mathcal{G}$ de modo que

$$
\left.r \operatorname{tip}\left(g^{\prime}\right) s=p \operatorname{tip}(g) \text { então } s \in \Gamma_{0} \text { e } g=g^{\prime}\right\}
$$

é uma base de Gröbner à direita para $I{ }^{3}$. Além disso, $\mathrm{rt \mathcal {G }}$ é um conjunto tip-reduzido formado por elementos uniformes. Dentre outras propriedades interessantes do conjunto $\mathrm{rt} \mathcal{G}$, destacaremos as seguintes:

1. se a cardinalidade de $\mathrm{rt \mathcal {G }}$ é finita, então o conjunto $\mathcal{G}$ também é finito.

2. se $\Lambda=R / I$ é uma álgebra de dimensão finita, então a cardinalidade de rtG é finita, com $|\operatorname{rt} \mathcal{G}| \leq \operatorname{dim}_{\mathrm{k}}(\Lambda) \cdot|\mathcal{G}|$, onde $|X|$ denota a cardinalidade do conjunto $X$.

Portanto, se $I$ é um ideal de $R=\mathrm{k} \Gamma$ tal que $R / I$ tem dimensão finita, então o item 2. garante que vI é um $R$-módulo finitamente gerado, para todo $v \in \Gamma_{0}$, uma vez que $v \mathrm{rtG}$ forma uma base de Gröbner à direita para vI.

\footnotetext{
${ }^{3}$ Estamos olhando $I$ como um $R$-submódulo de $R_{R}=\bigoplus_{v \in \Gamma_{0}} v R$.
} 
Finalizaremos esta introdução com dois resultados que serão úteis no decorrer do texto. O primeiro deles usa o conceito de termo líder para mostrar que $x R$ é um $R$-módulo projetivo, no caso em que $x$ é uniforme à direita e $(\mathcal{B},>)$ é uma base multiplicativa ordenada para $R=\mathrm{k} \Gamma$. Observe que este é um caso particular do teorema que afirma que $\mathrm{k} \Gamma$ é uma álgebra hereditária e assim todo ideal à direita (à esquerda) em $\mathrm{k} \Gamma$ é projetivo. Por outro lado, este teorema pode ser obtido como consequência da Proposição 4.12.

Lema 4.4 Seja $x$ um elemento de $R=\mathrm{k} \Gamma$ uniforme à direita, com $x v=x$ para algum $v \in \Gamma_{0}$. Então $\operatorname{tip}(x r)=\operatorname{tip}(x) \operatorname{tip}(r)$, para todo $r \in R \backslash\{0\}$ tal que $x r \neq 0$. Além disso, $x R \cong v R$ como $R$-módulos à direita.

Prova.

Se $x r \neq 0$ então $v r=r$. Assim, como $x v=x$, temos que tip $(x)=\operatorname{tip}(x) v$ e $\operatorname{tip}(r)=v \operatorname{tip}(r)$. De onde segue que tip $(x) \operatorname{tip}(r) \in \mathcal{B}$. Logo, se

$$
x=c_{1} \operatorname{tip}(x)+\sum_{i} c_{i} p_{i} \text { e } r=d_{1} \operatorname{tip}(r)+\sum_{j} d_{j} q_{j},
$$

onde $c_{i}, d_{j} \in \mathrm{k}$ e $p_{i}, q_{j} \in \mathcal{B}$, então

$$
x r=c_{1} d_{1} \operatorname{tip}(x) \operatorname{tip}(r)+\sum_{j} c_{1} d_{j} \operatorname{tip}(x) q_{j}+\sum_{i} d_{1} c_{i} p_{i} \operatorname{tip}(r)+\sum_{i, j} c_{i} d_{j} p_{i} q_{j}
$$

Mas como > é uma ordem admissível, temos as seguintes implicações:

- $\left[\operatorname{tip}(x)>p_{i}, \forall i\right] \wedge\left[\operatorname{tip}(r)>q_{j}, \forall j\right] \Rightarrow \operatorname{tip}(x) \operatorname{tip}(r)>p_{i} \operatorname{tip}(r)>p_{i} q_{j}, \forall i, \forall j$.

- $\operatorname{tip}(r)>q_{j}, \forall j \Rightarrow \operatorname{tip}(x) \operatorname{tip}(r)>\operatorname{tip}(x) q_{j}, \forall j$.

Isto mostra que tip $(x y)=\operatorname{tip}(x) \operatorname{tip}(r)$.

Para a segunda parte, definimos $\phi: v R \rightarrow x R$ por $\phi(v r)=x r$, para todo $r \in R$. É claro que $\phi$ é um morfismo de $R$-módulos que é sobrejetor. Mostremos que $\phi$ é injetor: suponha que $0=\phi(v r):=x r$. Pela primeira parte, $\operatorname{tip}(x) v \neq 0, v \operatorname{tip}(r) \neq 0$ se $v r \neq 0$. Assim, $\operatorname{tip}(x) \operatorname{tip}(r) \neq 0$ $\mathrm{e}$, portanto, $\operatorname{tip}(x r) \neq 0$. Isto contradiz a igualdade $x r=0 \mathrm{e}$, com isso, podemos concluir que $\phi$ é injetor.

Como resultado do lema acima, temos o seguinte

Corolario 4.5 Para todo $x \in R=\mathrm{k} \Gamma$, o $R$-módulo $x R$ é projetivo.

Prova.

Como todo elemento de $R$ é escrito, de forma única, como uma soma de elementos uniformes à direita, então o resultado segue.

É interessante para o que segue resumir estes dois resultados dizendo: se $x$ é um elemento de $R$ então $x R$ é um $R$-módulo projetivo dado por $x R=\bigoplus_{v \in \Gamma_{0}}(x v) R \cong \bigoplus_{v \in \Gamma_{0}^{\prime}} v R$, com $\Gamma_{0}^{\prime} \subseteq \Gamma_{0}$. 


\subsection{Exemplo de módulos com base ordenada}

Ao longo desta seção $R$ denota a álgebra de caminhos $\mathrm{k} \Gamma$ de um carcás finito, $\mathcal{B}$ a base usual de $R, \mathcal{I}$ um conjunto de índices e para cada $i \in \mathcal{I}, v_{i}$ é um vértice em $\Gamma$.

Definiremos uma base $\mathcal{C}$ para o $R$-módulo (à direita) projetivo $P=\bigoplus_{i \in \mathcal{I}} v_{i} R$ e, em seguida, uma boa ordem sobre tal base, a qual dependerá de uma ordem admissível sobre a base $\mathcal{B}$ de $R$ e de uma boa ordem sobre o conjunto de índices $\mathcal{I}$.

Definição 4.8 Seja $P=\bigoplus_{i \in \mathcal{I}} v_{i} R$ e para cada $j \in \mathcal{I}$ considere o conjunto

$$
\mathcal{C}_{j}:=\left\{x=\left(x_{i}\right)_{i \in \mathcal{I}} \in P: x_{j} \text { é um caminho em } R \text { com origem em } v_{j} \text { e } x_{i}=0 \text { se } i \neq j\right\} .
$$

Definimos $\mathcal{C}$ como a união dos $\mathcal{C}_{j}$ 's, com $j$ percorrendo o conjunto de índices $\mathcal{I}$.

Assim definido, o conjunto $\mathcal{C}$ forma uma k-base para o $R$-módulo $P=\bigoplus_{i \in \mathcal{I}} v_{i} R$. Além disso, dados $b \in \mathcal{B}$ e $x \in \mathcal{C}$, é claro que $x b \in \mathcal{C} \cup\{0\}$, ou seja, $\mathcal{C}$ é uma base coerente de $P=\bigoplus_{i \in \mathcal{I}} v_{i} R$. Por outro lado, se $>_{\mathcal{I}}$ é uma boa ordem para $\mathcal{I}$ e $>$ é uma ordem admissível para $\mathcal{B}$, então podemos definir uma ordem sobre $\mathcal{C}$ como segue:

Definição 4.9 (Ordem tip-índice) Sejam $x=\left(x_{j}\right)$ e $y=\left(y_{j}\right)$ dois elementos de $\mathcal{C}$. Diremos que $x \succ y$ se o caminho não nulo que aparece em $x$ é maior do que o caminho não nulo que aparece $y$ (com respeito a ordem admissivel $>$ sobre $\mathcal{B})$, ou, se estes caminhos forem iguais e a coordenada do elemento não nulo que ocorre em x é maior que a coordenada do elemento não nulo que ocorre em y (usando $\left.>_{\mathcal{I}}\right)$.

É claro que $\succ$ depende da escolha da boa ordem $>_{\mathcal{I}}$ para $\mathcal{I}$. Assim, sempre que usarmos um conjunto de índices $\mathcal{I}$ fixaremos alguma boa ordem $>_{\mathcal{I}}$. O próximo lema mostra que $\mathcal{C}$ é um conjunto bem ordenado com respeito a ordem $\succ$.

Lema 4.6 Sejam $\left(\mathcal{I},>_{\mathcal{I}}\right)$ um conjunto bem ordenado, $P=\bigoplus_{i \in \mathcal{I}} v_{i} R$ um $R$-módulo (com cada $\left.v_{i} \in \Gamma_{0}\right), \mathcal{C}=\bigcup_{i \in \mathcal{I}} \mathcal{C}_{i} e \succ$ como nas definições 4.8 e 4.9, respectivamente. Então $(\mathcal{C}, \succ)$ é um conjunto bem ordenado.

Prova.

Mostraremos apenas que qualquer subconjunto finito, $X=\left\{x^{1}, \ldots, x^{n}\right\}$, de $\mathcal{C}$ é totalmente ordenado com respeito a $\succ$. A prova é feita usando indução sobre o número de elementos em $X$. Para tanto, suponhamos que $x_{i_{j}}^{j}$ é o caminho não nulo que ocorre em $x^{j}$.

- O caso $n=1$ não há o que fazer!

- $n=2:$ se $x_{i_{1}}^{1} \neq x_{i_{2}}^{2}$ então $x_{i_{1}}^{1}>x_{i_{2}}^{2}$ ou $x_{i_{2}}^{2}>x_{i_{1}}^{1}$ o que implica que $x^{1} \succ x^{2}$ ou $x^{2} \succ x^{1}$, respectivamente. Se $x_{i_{1}}^{1}=x_{i_{2}}^{2}$, usamos a boa ordem $>_{\mathcal{I}}$ para decidir se $x^{1} \succ x^{2}$ ou se $x^{2} \succ x^{1}$. 
- $n=k+1$, para $k \geq 2$ : por hipótese de indução o conjunto $\left\{x^{1}, \ldots, x^{k}\right\}$ é totalmente ordenado. Agora, pelo caso $n=2, x^{k+1}$ é comparável com cada $x^{j}$, com $j=1, \ldots, k$ e, portanto, $\left\{x^{1}, \ldots, x^{k+1}\right\}$ é um conjunto totalmente ordenado.

Para que $(\mathcal{C}, \succ)$ seja uma base ordenada de $P=\bigoplus_{i \in \mathcal{I}} v_{i} R$, resta verificar que $\succ$ é uma ordem admissível, e este é o resultado do próximo lema.

Lema 4.7 Nas mesmas hipóteses do Lema 4.6, temos que $\succ$ é uma ordem admissível sobre $\mathcal{C}$.

Prova.

Consideremos $x, y \in \mathcal{C}$ e $p, q \in \mathcal{B}$. Vamos mostrar que se $x \succ y$ então $x p \succ y p$ (sempre que $x p \neq 0 \neq y p)$ e que se $p>q$ então $x p \succ x q$ (sempre que $x p \neq 0 \neq x q$ ). De fato, primeiramente suponhamos que $x \succ y$ e que $x p \neq 0 \neq y p$. Assim, por definição, ou a coordenada (não nula) $x_{j}$ que ocorre em $x$ é maior que a coordenada $y_{i}$ que ocorre em $y$ ou elas são iguais e $j>_{\mathcal{I}} i$. Se ocorrer o primeiro caso, como $x_{j} p \neq 0 \neq y_{i} p$ e $>$ é uma ordem admissível sobre $\mathcal{B}$, segue que $x_{j} p>y_{i} p$ e, portanto, $x p \succ y p$. Por outro lado, se ocorrer o segundo caso, teremos que $x_{j} p=y_{i} p$ é a coordenada que ocorre em $x p$ e em yp. Como estas ocorrem nas posições $j$ e $i$, respectivamente, e $j>_{\mathcal{I}} i$, novamente teremos $x p \succ y p$. Isto conclui a prova da primeira parte. A prova da segunda parte é análoga.

Assim, concluímos que $(\mathcal{C}, \succ)$ é uma base ordenada para $P=\bigoplus_{i \in \mathcal{I}} v_{i} R$ e, portanto, todos os resultados obtidos em [12] para a teoria de $R$-módulos com base ordenada poderão ser utilizados para a terna $(P, \mathcal{C}, \succ)$.

Encerraremos esta seção definindo novamente, para efeito de uso posterior, os conceito de termo líder de um elemento de $P$ e de conjunto tip-reduzido, de forma equivalente àquela feita na Definição 4.7. Restrigiremos nossa atenção aos elementos uniformes à direita, pois estes serão suficientes para resultados posteriores.

Definição 4.10 Seja $w=\left(w_{i}\right)$ um elemento uniforme à direita, não nulo, de $P=\bigoplus_{i \in \mathcal{I}} v_{i} R$. Então tip $(w)$ é o elemento de $\mathcal{C}$ cuja k-ésima coordenada é o caminho tip $\left(w_{k}\right) \in \mathcal{B}$ que satisfaz as seguintes condições:

T1. $\operatorname{tip}\left(w_{k}\right) \geq \operatorname{tip}\left(w_{i}\right)$, para todo $w_{i}$ não nulo;

T2. $k \geq_{\mathcal{I}} i$ para todo $i$ tal que $\operatorname{tip}\left(w_{k}\right)=\operatorname{tip}\left(w_{i}\right)$.

Em outras palavras, o tip $(w)$ é o elemento com maior índice entre as entradas com termo líder maximal.

Nessas condições denotaremos o caminho tip $\left(w_{k}\right)$ por tippath $(w)$ e o indice $k$ por tipcoord $(w)$.

Pela definição acima, obtemos propriedades semelhantes àquelas já conhecidas. 
Lema 4.8 Sejam $v, w \in P=\bigoplus_{i \in \mathcal{I}} v_{i} R$ uniformes à direita e $p$ e $q$ caminhos em $\mathcal{B}$. Assim:

(a) $\operatorname{tippath}(w p)=\operatorname{tippath}(w) p, \operatorname{tipcoord}(w p)=\operatorname{tipcoord}(w)$ e $\operatorname{tip}(w p)=\operatorname{tip}(w) p$, sempre que $\operatorname{tip}(w) p \neq 0$.

(b) se $\operatorname{tip}(v) \succ \operatorname{tip}(w)$ então $\operatorname{tip}(v p) \succ \operatorname{tip}(w p)$, sempre que $\operatorname{tip}(v) p \neq 0$ e $\operatorname{tip}(w) p \neq 0$.

Prova.

(a) Se tip $(w) p \neq 0$ então tip $\left(w_{k}\right) p$ é a coordenada não nula de tip $(w) p$, onde $k=\operatorname{tipcoord}(w)$. Mas como sabemos do Lema $4.4 \operatorname{tip}\left(w_{k} p\right)=\operatorname{tip}\left(w_{k}\right) p$, basta mostrarmos que tip $\left(w_{k} p\right)$ satisfaz as condições T1 e T2 da Definição 4.10. Dado que tip $\left(w_{i}\right) \leq \operatorname{tip}\left(w_{k}\right)$, para todo $i$, a condição $T 1$ segue da desigualdade

$$
\operatorname{tip}\left(w_{i} p\right)=\operatorname{tip}\left(w_{i}\right) p \leq \operatorname{tip}\left(w_{k}\right) p=\operatorname{tip}\left(w_{k} p\right), \text { para todo } w_{i} \neq 0 .
$$

E Se tip $\left(w_{i} p\right)=\operatorname{tip}\left(w_{k} p\right)$, então $\operatorname{tip}\left(w_{i}\right) p=\operatorname{tip}\left(w_{k}\right) p$ e, portanto, $\operatorname{tip}\left(w_{i}\right)=\operatorname{tip}\left(w_{k}\right)$. De onde segue que $k \geq_{\mathcal{I}} i$, provando assim a condição T2.

(b) Segue diretamente do item (a) e do fato de $\succ$ ser uma ordem admissível sobre $\mathcal{C}$ (Lema 4.7).

Antes de lembrarmos da noção de conjunto tip-reduzido à direita, reescreveremos o conceito de divisão à esquerda usando a notação acima:

○ se $x$ e $y$ são elementos da base $\mathcal{C}$, então dizemos que $x$ divide $y$ à esquerda, e escrevemos $\left.x\right|_{e} y$, quando existe um caminho $p \in \mathcal{B}$ tal que $y=x p$. E este é o caso se, e somente se, $\left.\operatorname{tippath}(x)\right|_{e} \operatorname{tippath}(y)$ e $\operatorname{tipcoord}(x)=\operatorname{tipcoord}(y)$.

Definição 4.11 Seja $X$ um subconjunto de $P=\bigoplus_{i \in \mathcal{I}} v_{i} R$ formado por elementos, não nulos, uniformes à direita. Dizemos que $X$ é tip-reduzido à direita se dados $v, w \in X$ tais que $\left.\operatorname{tip}(v)\right|_{e} \operatorname{tip}(w)$, então $v=w$.

A próxima proposição mostra, de certa forma, que sempre podemos "tip-reduzir" um subconjunto de $P$ formado por elementos uniformes à direita. É importante observar que a demonstração, que é bem parecida com aquela apresentada para o Lema 4.3, mostra efetivamente como "tipreduzir" um dado conjunto.

Proposição 4.9 Seja $X$ um subconjunto de $P=\bigoplus_{i \in \mathcal{I}} v_{i} R$ formado por elementos, não nulos, uniformes à direita. Então existe um subconjunto, $Y$, de $P$ composto por elementos uniformes à direita tal que $Y$ é tip-reduzido e o R-submódulo de $P$ gerado por $Y$ é igual ao gerado por $X$.

Prova. 
Sejam $M$ o $R$-submódulo de $P$ gerado por $X, T:=\{\operatorname{tip}(m): m \in M \backslash\{0\}$ uniforme à direita $\}$ e $T^{*}:=\left\{t \in T:\right.$ se existe $t^{\prime} \in T$ tal que $\left.t^{\prime}\right|_{e} t$ então $\left.t^{\prime}=t\right\}$. É claro que tip $(X):=\{\operatorname{tip}(x): x \in X\}$ é um subconjunto de $T$ e, portanto, $T \neq \emptyset$. Para cada $t \in T^{*}$, escolhamos um elemento uniforme à direita $y_{t}$ em $M$ tal que tip $\left(y_{t}\right)=t$ e consideremos $Y:=\left\{y_{t}: t \in T^{*}\right\}$. Com isso, $Y$ é um conjunto tip-reduzido composto por elementos uniformes à direita e note que o $R$-submódulo $N$ de $P$ gerado por $Y$ está contido em $M$.

Observemos que para mostrarmos que $M \subseteq N$, basta verificar que todos os elementos uniformes à direita de $M$ pertencem a $N$, uma vez todo elemento $m \in M$ pode ser escrito na forma $m=$ $\sum_{v \in \Gamma_{0}} m v$. De fato, suponhamos que existe um elemento uniforme à direita $m \in M$ tal que $m \in M \backslash N$. Podemos escolher $m \in M \backslash N$ com a propriedade que tip $(m)$ seja minimal com respeito a ordem $\succ$. Logo $m \notin Y$ e, portanto, temos uma das seguintes condições:

- $\operatorname{tip}(m) \neq \operatorname{tip}\left(y_{t}\right)$, para todo $t \in T^{*}$, o que implica que existe $t_{1} \in T$ tal que $\left.t_{1}\right|_{e} \operatorname{tip}(m)$. Se $t_{1} \notin T^{*}$, então existe $t_{2} \in T$ tal que $\left.t_{2}\right|_{e} t_{1}$. Se $t_{2} \notin T^{*}$, então existe $t_{3} \in T$ tal que $\left.t_{3}\right|_{e} t_{2}$. Continuando este processo, construímos uma cadeia descendente da forma

$$
t_{1} \succ t_{2} \succ \cdots \succ t_{k} \succ \cdots
$$

Como $(\mathcal{C}, \succ)$ é um conjunto bem ordenado, então existe $s$ tal que $t_{s}=t_{s+j}, \forall j \geq 0$. De onde segue que existe $s$ tal que $t_{s} \in T^{*}$ e $\left.t_{s}\right|_{e} \operatorname{tip}(m)$.

- $\operatorname{tip}(m)=\operatorname{tip}\left(y_{t}\right):=t$, para $\operatorname{algum} t \in T^{*} .\left.\mathrm{E} \operatorname{assim} t\right|_{e} \operatorname{tip}(m)$.

Em qualquer dos casos existe $t \in T^{*}$ tal que $\left.t\right|_{e}$ tip $(m)$. Então escrevendo $m=\lambda_{0} m_{0}+\sum_{i} \lambda_{i} m_{i}$, onde $m_{0} \succ m_{i}, \forall i \neq 0$, cada $\lambda_{i} \in \mathbf{k} \backslash\{0\}$ e cada $m_{i} \in \mathcal{C}$, teremos que existe $p \in \mathcal{B}$ de forma que

$$
m=\lambda_{0} \operatorname{tip}\left(y_{t}\right) p+\sum_{i} \lambda_{i} m_{i}, \operatorname{com} y_{t} \in N \operatorname{etip}\left(y_{t}\right)=t
$$

Logo $\operatorname{tip}(m) \succ \operatorname{tip}\left(m-\lambda_{0} \operatorname{tip}\left(y_{t}\right) p\right)$ e pela minimalidade do tip $(m)$ segue que $m-\lambda_{0} \operatorname{tip}\left(y_{t}\right) p \in N$, e portanto $m \in N$, contradizendo nossa hipótese.

O próximo resultado garante, em particular, que existe um algoritmo finito no caso em que o conjunto $X$, da proposição anterior, é um conjunto finito.

Corolario 4.10 Nas mesmas condições da Proposição 4.9, o conjunto $Y$ é finito sempre que $X$ for finito.

Prova.

Seja $X:=\left\{x_{1}, \ldots, x_{n}\right\}$. Se $X$ não é tip-reduzido, então existem $i$ e $j$ tais que $i \neq j$ e $\left.\operatorname{tip}\left(x_{i}\right)\right|_{e} \operatorname{tip}\left(x_{j}\right)$. Consideremos $X_{1}=\left\{x_{1}, \ldots, x_{j-1}, x_{j^{\prime}}, x_{j+1}, \ldots, x_{n}\right\}$, onde $x_{j^{\prime}}=x_{j}-c x_{i} p$ para algum $c \in$ k e algum $p \in \mathcal{B}$. Note que o $R$-submódulo de $P$ gerado por $X_{1}$ é igual ao gerado por $X$. Além disso, $x_{j^{\prime}}$ é uniforme à direita e tip $\left(x_{j}\right) \succ \operatorname{tip}\left(x_{j^{\prime}}\right)$. Se $X_{1}$ não é tip-reduzido, construímos 
$X_{2}$ de forma análoga. Este processo pára num número finito de passos, pois $\succ$ é uma boa ordem para $\mathcal{C}$. E então $Y=X_{s}$ é um conjunto finito tip-reduzido à direita e o $R$-submódulo de $P$ gerado por $Y$ é igual ao gerado por $X$.

Como ilustração do processo acima faremos o seguinte

Exemplo 4.6 Seja $\Gamma$ o carcás

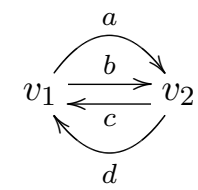

com ordem grau-lexicográfica $>$ sobre $\mathcal{B}$ definida a partir de $v_{1}<v_{2}<a<b<c<d$. Sejam

$$
P=v_{1} R \oplus v_{1} R \oplus v_{2} R \oplus v_{2} R
$$

e a ordem $\succ$ induzida por $\left(v_{1}, 0,0,0\right) \succ\left(0, v_{1}, 0,0\right) \succ\left(0,0, v_{2}, 0\right) \succ\left(0,0,0, v_{2}\right)$. Consideremos $X=\left\{f_{1}, f_{2}, f_{3}\right\}$, onde $f_{1}=(a c, b c a c+a c a c, 0, c a c), f_{2}=\left(v_{1}, b c+a c, d, 0\right)$ e $f_{3}=(a, b, d a+c b, d a)$. Observe que $f_{1}=f_{1} v_{1}, f_{2}=f_{2} v_{1}$ e $f_{3}=f_{3} v_{2}$. Além disso, como

- $a c<c a c<a c a c<b c a c \Rightarrow \operatorname{tip}\left(f_{1}\right)=(0, b c a c, 0,0)$

- $v_{1}<d<a c<b c \Rightarrow \operatorname{tip}\left(f_{2}\right)=(0, b c, 0,0)$

- $a<b<c b<d a$ e $\left(0,0, v_{2}, 0\right) \succ\left(0,0,0, v_{2}\right) \Rightarrow \operatorname{tip}\left(f_{3}\right)=(0,0, d a, 0)$

temos que $\operatorname{tip}\left(f_{1}\right)=\operatorname{tip}\left(f_{2}\right) a c$, ou seja, $\left.\operatorname{tip}\left(f_{2}\right)\right|_{e} \operatorname{tip}\left(f_{1}\right)$. Então trocamos $f_{1}$ por $g_{1}:=f_{1}-f_{2} a c=$ $(0,0,-d a c, c a c)$ e consideramos $X_{1}=\left\{g_{1}, f_{2}, f_{3}\right\}$. Agora, dado que tip $\left(g_{1}\right)=(0,0, d a c, 0)$ temos que tip $\left.\left(f_{3}\right)\right|_{e} \operatorname{tip}\left(g_{1}\right)$ e assim trocamos $g_{1}$ por $g_{2}=g_{1}-(-1) f_{3} c=(a c, b c, c b c, c a c+d a c)$. Mas como $\operatorname{tip}\left(g_{2}\right)=(0,0,0, d a c)$, segue que o conjunto $Y=X_{2}=\left\{g_{2}, f_{2}, f_{3}\right\}$ é tip-reduzido à direita. Além disso, o $R$-submódulo de $P=v_{1} R \oplus v_{1} R \oplus v_{2} R \oplus v_{2} R$ gerado por $X$ ou por $X_{1}$ ou por $X_{2}$ é igual a $g_{2} R \oplus f_{2} R \oplus f_{3} R$.

Concluiremos esta seção com os seguintes resultados importantes.

Lema 4.11 ([12]) Se $\mathcal{G}$ é um subconjunto tip-reduzido de $P=\bigoplus_{i \in \mathcal{I}} v_{i} R$ formado por elementos uniformes à direita, então o submódulo de $P$ gerado por $\mathcal{G}$ é o $R$-módulo projetivo $\bigoplus_{g \in \mathcal{G}} g R$.

Assim obtemos um resultado fundamental, que será usado livremente no que segue.

Proposição 4.12 Seja $Q$ um submódulo de um $R$-módulo projetivo $P=\bigoplus_{i \in \mathcal{I}} v_{i} R$, onde $\mathcal{I}$ é um conjunto de índices e cada $v_{i}$ é um vértice do carcás $\Gamma$. Então existe uma base de Gröbner à direita $\left\{f_{j}\right\}_{j \in \mathcal{J}}$ uniforme à direita tip-reduzida de $Q$. Além disso $Q=\bigoplus_{j \in \mathcal{J}} f_{j} R$, qualquer que seja essa base. 
Prova.

Seja $(\mathcal{C}, \succ)$ uma base ordenada para $P$. Então pelo Lema 4.3 todo submódulo de $P$ possui uma base de Gröbner à direita uniforme tip-reduzida. A última parte segue do lema precedente.

Corolario 4.13 A álgebra de caminhos de $\Gamma$ é uma álgebra hereditária.

Para finalizar a seção, faremos uma observação que será de suma importância no que segue.

Observação 4.3 Da Observação 4.2 segue que o conjunto $\left\{f_{j}\right\}_{j \in \mathcal{J}}$ da proposição anterior pode ser escolhido enumerável.

\subsection{Construindo uma resolução projetiva}

O principal objetivo desta seção é exibir um procedimento, apresentado em [13], que constrói resoluções projetivas de módulos sobre quocientes de álgebras de caminhos. Para isto faremos uso dos conceitos das seções precedentes e da teoria para construção de resoluções projetivas apresentada em [14].

Assumamos nesta seção que $R=\mathrm{k} \Gamma$ é a álgebra de caminhos de um carcás finito sobre o corpo k, $I$ é um ideal de $R$ tal que $I \subseteq J^{2}$, onde $J$ denota o ideal de $R$ gerado pelas flechas de $\Gamma$, e que $M_{\Lambda}$ é um $\Lambda$-módulo à direita, onde $\Lambda$ é álgebra quociente $R / I$.

Iniciaremos com a construção (feita em [14]) de uma resolução projetiva para o $\Lambda$-módulo $M_{\Lambda}$.

Escolha uma família $\left\{f_{i}^{0}\right\}_{i \in T_{0}}$ de elementos de $R$ tal que existe um epimorfismo do $\Lambda$-módulo $\bigoplus_{i \in T_{0}} f_{i}^{0} R / \bigoplus_{i \in T_{0}} f_{i}^{0} I$ em $M_{\Lambda}$. Podemos supor sem perda de generalidade que cada $f_{i}^{0}$ é vértice em $\Gamma$ (com possíveis repetições). Assim, temos o seguinte diagrama de $R$-módulos

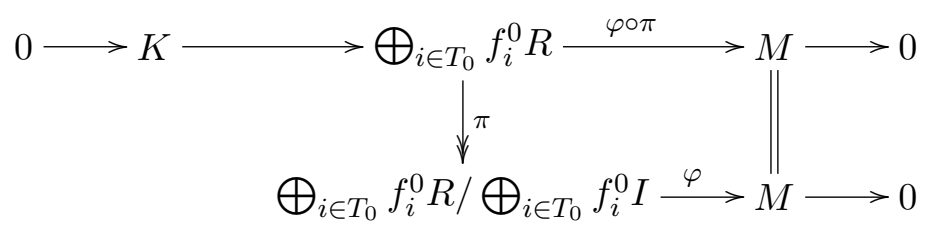

onde $\pi$ é a projeção canônica e $K=$ Nuc $(\varphi \circ \pi)$. Logo, como $K$ é um submódulo de $\bigoplus_{i \in T_{0}} f_{i}^{0} R$, então pela Proposição 4.12 existe um conjunto tip-reduzido, $\left\{f_{j}^{1^{*}}\right\}_{j}$, de elementos (uniformes à

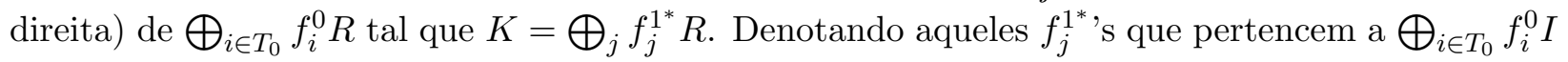
por $f_{j}^{1}$ 's e os outros por $f_{j}^{1}$ 's, obtemos uma apresentação para o $R$-módulo $M_{\Lambda}$ da seguinte forma:

$$
0 \longrightarrow\left(\bigoplus_{i \in T_{1}} f_{i}^{1} R\right) \oplus\left(\bigoplus_{j \in U_{1}} f_{j}^{1^{\prime}} R\right) \stackrel{H^{1}}{\longrightarrow} \bigoplus_{i \in T_{0}} f_{i}^{0} R \stackrel{\varphi \circ \pi}{\longrightarrow} M \longrightarrow 0
$$

onde

(i) $H^{1}$ é uma inclusão,

(ii) os $f_{i}^{0}$ 's, $f_{i}^{1}$ 's e $f_{i}^{1^{\prime}}$ 's são uniformes à direita, 
(iii) $f_{j}^{1^{\prime}} \in \bigoplus_{i \in T_{0}} f_{i}^{0} I$, para cada $j \in U_{1}$,

(iv) o conjunto $\left\{f_{i}^{1}\right\}_{i \in T_{1}} \cup\left\{f_{j}^{1^{\prime}}\right\}_{j \in U_{1}}$ é tip-reduzido.

Para a construção dos $f^{2^{*}}$ 's consideremos a intersecção $\left(\bigoplus_{i \in T_{1}} f_{i}^{1} R\right) \cap\left(\bigoplus_{i \in T_{0}} f_{i}^{0} I\right)$. Se ela for igual a zero, paramos o processo. Caso contrário, existe um conjunto $\left\{f_{j}^{2^{*}}\right\}_{j}$ de elementos uniformes à direita (tip-reduzida) em $\bigoplus_{i \in T_{1}} f_{i}^{1} R$ tal que $\left(\bigoplus_{i \in T_{1}} f_{i}^{1} R\right) \cap\left(\bigoplus_{i \in T_{0}} f_{i}^{0} I\right)=\bigoplus_{j} f_{j}^{2^{*}} R$. Novamente, descartamos aqueles elementos $f_{j}^{2^{*}}$ 's que estão em $\bigoplus_{i \in T_{1}} f_{i}^{1} I$ e denotamos por $\left\{f_{i}^{2}\right\}_{i \in T_{2}}$ aqueles $f_{j}^{2^{*}}$ 's que não estão em $\bigoplus_{i \in T_{1}} f_{i}^{1} I$. Se ocorrer o caso de todos os $f_{j}^{2^{*}}$ 's pertencerem a $\bigoplus_{i \in T_{1}} f_{i}^{1} I$, o conjunto $T_{2}=\emptyset$ e assim paramos a construção. Logo, de forma indutiva constrói-se a família de elementos $\left\{f_{i}^{k}\right\}_{i \in T_{k}}$, para qualquer $k \geq 3$, onde cada $f_{i}^{k} \in\left(\bigoplus_{i \in T_{k-1}} f_{i}^{k-1} R\right) \backslash\left(\bigoplus_{i \in T_{k-1}} f_{i}^{k-1} I\right)$.

Como o leitor pode observar, no processo acima se utiliza apenas a existência de uma família $\left\{f_{j}\right\}_{j \in \mathcal{J}}$ de elementos uniformes à direita (ver Proposição 4.12).

Da construção obtemos a seguinte cadeia de inclusões

$$
\cdots \subseteq \bigoplus_{i \in T_{n}} f_{i}^{n} R \subseteq \bigoplus_{i \in T_{n-1}} f_{i}^{n-1} R \subseteq \cdots \subseteq \bigoplus_{i \in T_{1}} f_{i}^{1} R \subseteq \bigoplus_{i \in T_{0}} f_{i}^{0} R
$$

Além disso, temos o seguinte resultado.

Teorema 4.14 ([14]) Para cada $n \geq 0$, sejam $P_{n}=\bigoplus_{i \in T_{n}} f_{i}^{n} R / \bigoplus_{i \in T_{n}} f_{i}^{n} I$ e $\delta^{n}: P_{n} \rightarrow P_{n-1}$ o homomorfismo de $\Lambda$-módulos induzido pela inclusão $\bigoplus_{i \in T_{n}} f_{i}^{n} R \subseteq \bigoplus_{i \in T_{n-1}} f_{i}^{n-1} R$. Então,

$$
(\mathcal{P}, \delta): \quad \cdots \longrightarrow P_{n} \stackrel{\delta^{n}}{\longrightarrow} P_{n-1} \rightarrow \cdots \rightarrow P_{1} \stackrel{\delta^{1}}{\longrightarrow} P_{0} \stackrel{\varphi}{\longrightarrow} M_{\Lambda} \longrightarrow 0
$$

é uma resolução projetiva de $M_{\Lambda}$.

Em [14] mostra-se também que esta resolução $(\mathcal{P}, \delta)$ é finitamente gerada sempre que $\Lambda$ for uma álgebra noetheriana, $I$ um $R$-módulo à direita finitamente gerado e $M_{\Lambda}$ um $\Lambda$-módulo finitamente gerado (Teorema 1.3 de [14]). Em particular, se $I$ é um ideal de $R$ tal que $J^{n} \subseteq I \subseteq J^{2}$, para algum $n \geq 2$, e $M$ é um $\Lambda$-módulo de dimensão finita, então a resolução $(\mathcal{P}, \delta)$ é finitamente gerada.

Além disso, se $\Lambda=\mathrm{k} \Gamma / I$ é uma álgebra graduada, com $I \subseteq J^{2}$ sendo um ideal graduado, e $M$ é um $\Lambda$-módulo graduado, então a resolução $(\mathcal{P}, \delta)$ pode ser escolhida como sendo uma resolução graduada (Proposição 1.5 de [14]).

Finalmente, visto que $(\mathcal{P}, \delta)$ não é necessariamente minimal, uma questão natural seria descobrir em que situações podemos "ajustar" tal resolução de forma que ela torne-se minimal. Ainda em [14], mostra-se que isso é possível sempre que $J^{n} \subseteq I \subseteq J^{2}$, para algum $n \geq 2$, ou sempre que $\Lambda$ é uma álgebra graduada e $M$ um $\Lambda$-módulo graduado.

Baseado na construção acima, queremos encontrar um procedimento que encontre uma resolução projetiva para um dado $\Lambda$-módulo e para isto, faz-se necessário iniciarmos com uma apresentação projetiva - sobre a álgebra de caminhos $R$ - para o dado $\Lambda$-módulo.

Sejam $I \subseteq J^{2}$ um ideal da álgebra de caminhos $R=\mathrm{k} \Gamma, \Lambda=R / I, M$ um $\Lambda$-módulo à direita e $\mathcal{G}=\left\{g_{i}\right\}_{i \in \mathcal{I}}$ uma base de Gröbner tip-reduzida uniforme para o ideal $I$, com respeito a alguma 
ordem admissível $>$ sobre a base usual $\mathcal{B}$ de $R$. Como sabemos, existe uma apresentação de $M$ sobre $R$ da forma

$$
0 \longrightarrow\left(\bigoplus_{i \in T_{1}} f_{i}^{1} R\right) \oplus\left(\bigoplus_{j \in U_{1}} f_{j}^{1^{\prime}} R\right) \stackrel{H^{1}}{\longrightarrow} \bigoplus_{i \in T_{0}} f_{i}^{0} R \stackrel{\varphi \circ \pi}{\longrightarrow} M \longrightarrow 0
$$

onde

(i) $H^{1}$ é uma inclusão,

(ii) os $f_{i}^{0}$ 's, $f_{i}^{1}$ 's e $f_{i}^{1^{\prime}}$ 's são uniformes à direita,

(iii) $f_{j}^{1^{\prime}} \in \bigoplus_{i \in T_{0}} f_{i}^{0} I$, para cada $j \in U_{1}$,

(iv) o conjunto $\left\{f_{i}^{1}\right\}_{i \in T_{1}} \cup\left\{f_{j}^{1^{\prime}}\right\}_{j \in U_{1}}$ é tip-reduzido,

(v) $T_{1}$ é um conjunto enumerável. (Veja Observação 4.3)

Queremos construir conjuntos $\left\{f_{i}^{2}\right\}_{i \in T_{2}}$ e $\left\{f_{i}^{2^{\prime}}\right\}_{i \in U_{2}}$ tais que $\left\{f_{i}^{2}\right\}_{i \in T_{2}} \cup\left\{f_{i}^{2^{\prime}}\right\}_{i \in U_{2}}$ seja um conjunto uniforme à direita, tip-reduzido, cada $f_{i}^{2^{\prime}}$ pertence a $\bigoplus_{i \in T_{1}} f_{i}^{1} I, T_{2}$ seja um conjunto enumerável e

$$
0 \longrightarrow\left(\bigoplus_{i \in T_{2}} f_{i}^{2} R\right) \oplus\left(\bigoplus_{j \in U_{2}} f_{j}^{2^{\prime}} R\right) \stackrel{H^{2}}{\longrightarrow} \bigoplus_{i \in T_{1}} f_{i}^{1} R \longrightarrow \Omega_{\Lambda}^{1}(M) \longrightarrow 0
$$

seja uma sequência exata de $R$-módulos onde $H^{2}$ é uma inclusão e $\Omega_{\Lambda}^{1}(M)$ é o núcleo do epimorfismo $\varphi: \bigoplus_{i \in T_{0}} f_{i}^{0} R / \bigoplus_{i \in T_{0}} f_{i}^{0} I \longrightarrow M$.

Antes da construção dos $f_{i}^{2}$ 's e dos $f_{i}^{2^{\prime}}$ 's, precisamos de algumas definições preliminares.

Definição 4.12 Sejam $\mathcal{G}=\left\{g_{i}\right\}_{i \in \mathcal{I}}$ uma base de Gröbner tip-reduzida para I e $p$ um caminho não trivial em $\Gamma$. Definimos $X(p)$ como o conjunto dos caminhos q que possui as seguintes propriedades:

(1) $\left.p\right|_{e} q$;

(2) Existe $g_{j} \in \mathcal{G}$ tal que tip $\left.\left(g_{j}\right)\right|_{d} q$, isto é, $q=q^{\prime} \operatorname{tip}\left(g_{j}\right)$ para algum $q^{\prime} \in \mathcal{B}$;

(3) Se existem $r, s \in \mathcal{B}$ e $g_{i} \in \mathcal{G}$ tais que $q=r \operatorname{tip}\left(g_{i}\right) s$, então $s$ é um vértice.

Observe que a existência do $g_{j}$ e do $g_{i}$ nas propriedades (2) e (3) da definição acima, implica que $i=j$, pois o conjunto $\left\{g_{t}\right\}_{t \in \mathcal{I}}$ é tip-reduzido. Se $q \in X(p)$ e $q=q^{\prime} \operatorname{tip}\left(g_{i}\right)$, chamamos $g_{i}$ da relação final de $q$.

Podemos ilustrar as propriedades (1) e (2) da definição de $X(p)$ por meio de uma das formas

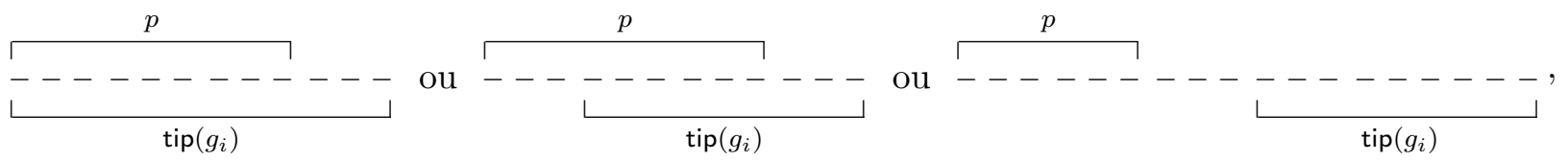

onde o caminho $q$ é indicado pela linha tracejada. 
Como veremos mais adiante os $f_{i}^{2}$ 's estarão associados a um dos dois primeiros diagramas e os $f_{i}^{2^{\prime}}$ 's ao terceiro diagrama. Assim, é interessante dividir o conjunto $X(p)$ nos dois subconjuntos (disjuntos):

$$
O(p):=\{q \in X(p): p \text { e o tip da relação final de } q \text { se sobrepõem }\} \text { e } N(p):=X(p) \backslash O(p) \text {. }
$$

Dessa forma, um elemento $q \in O(p)$ pode ser descrito pelo seguinte diagrama

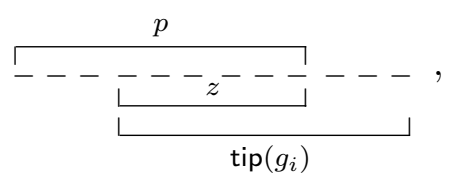

onde $1 \leq \ell(z) \leq \ell(p)$ e o caminho $q$ é indicado pela linha tracejada. Por outro lado, um elemento $q \in N(p)$ pode ser representado pelo diagrama

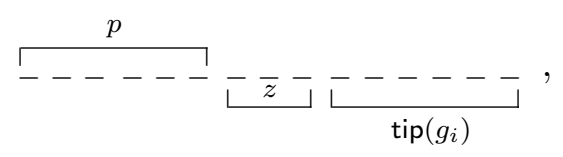

onde $z$ é um caminho de comprimento maior ou igual a zero.

O exemplo abaixo ilustra como encontrar os conjuntos $X, N$ e $O$ definidos acima.

Exemplo 4.7 Sejam $\Gamma$ o carcás

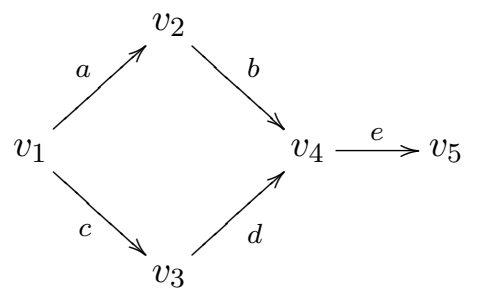

e I o ideal de $\mathrm{k} \Gamma$ gerado pelo conjunto $\{a b-c d, b e\}$. Queremos encontrar os conjuntos $X(a), X(b)$ e $X(c)$, com respeito as ordens $>_{1}$ e $>_{2}$ do Exemplo 4.3.

(a) Seja $>_{1}$ a ordem grau-lexicográfica induzida por $a>\ldots>e>v_{1}>\ldots>v_{5}$, então $\mathcal{G}=$ $\left\{g_{1}=a b-c d, g_{2}=b e, g_{3}=c d e\right\}$ é uma base Gröbner para o ideal I. Além disso, é fácil ver que esta base é tip-reduzida.

Por definição, os elementos de $X(a)$ são os caminhos de $\mathcal{B}$ tais que

(1) $\left.a\right|_{e} q$;

(2) Existe $g_{j} \in \mathcal{G}$ tal que $\left.\operatorname{tip}\left(g_{j}\right)\right|_{d} q$, isto é, $q=q^{\prime} \operatorname{tip}\left(g_{j}\right)$ para algum $q^{\prime} \in \mathcal{B}$;

(3) Se existem $r, s \in \mathcal{B}$ e $g_{i} \in \mathcal{G}$ tais que $q=r \operatorname{tip}\left(g_{i}\right) s$, então s é um vértice. 
De (1) segue que $q \in\{a, a b, a b e\}$. Mas como $\operatorname{tip}(\mathcal{G})=\{a b, b e, c d e\}$, da propriedade (2) temos que $q \neq a$. Por outro lado, observe que abe $=r(a b) s$, onde $s=e, r=v_{1}$ e ab=tip $\left(g_{1}\right)$, isto é, abe não satisfaz a condição (3). Portanto, $X(a)=\{a b\}$. Além disso, como $p=a$ e o termo líder da relação final de $q=a b$, que é o próprio ab, se sobrepõem, então $O(a)=\{a b\}=X(a)$ e $N(a)=\emptyset$.

De forma análoga conclui-se que $X(c)=\{c d e\}=O(c)$ e $N(c)=\emptyset$. Para encontrar $X(b)$, observe que o único elemento de $\mathcal{B}$ que satisfaz (1) é o caminho $q=$ be. Além disso, tomando $j=2$ na propriedade (2), teremos que be $=($ be $) v_{5}=\operatorname{tip}\left(g_{3}\right) v_{5}$, isto é, be satisfaz a condição (2). Finalmente, a condição (3) é satisfeita trivialmente e, portanto, $X(b)=\{b e\}$

(b) Seja $>_{2}$ a ordem grau-lexicográfica induzida por $e>\ldots>a>v_{1}>\ldots>v_{5}$. Então $\mathcal{G}=\{a b-c d, b e\}$ é base de Gröbner tip-reduzida e tip $(\mathcal{G})=\{c d, b e\}$. Se $q \in X(a)$, então $q \in\{a, a b, a b e\}$ e pela condição (2) segue que $q=a b e$. Além disso, é claro que abe satisfaz a condição (3). Portanto, $X(a)=\{a b e\}$. Por outro lado, é fácil verificar que $O(a)=\emptyset e$ $N(a)=X(a)$. Fica como exercício para o leitor verificar que $X(b)=\{b e\}=O(b), X(c)=$ $\{c d\}=O(c)$ e $N(b)=\emptyset=N(c)$.

Definiremos agora $T_{2}$, o conjunto de índices para os $f_{i}^{2}$ 's:

- $T_{2}:=\left\{(i, q): i \in T_{1}\right.$ e $\left.q \in O\left(\operatorname{tippath}\left(f_{i}^{1}\right)\right)\right\}$

Como $T_{1}$ e $\mathcal{B}$ são conjuntos enumeráveis, segue que $T_{2}$ também o é. Uma vez definido $T_{2}$, podemos construir o elemento $f_{s}^{2}$, para um dado $s \in T_{2}$. Para tanto, suponha que $s=(i, q)$ e que $\operatorname{tipcoord}\left(f_{i}^{1}\right)=i^{*}$. Dadas as definições de $T_{2}$ e de $O$, vemos que existem $p, q^{\prime} \in \mathcal{B}$ e $g_{j} \in \mathcal{G}$ tais que $q=\operatorname{tippath}\left(f_{i}^{1}\right) p=q^{\prime} \operatorname{tip}\left(g_{j}\right)$. Desta igualdade, é claro que $\mathrm{t}(p)=\mathrm{t}(q)=\mathrm{t}\left(g_{j}\right)$.

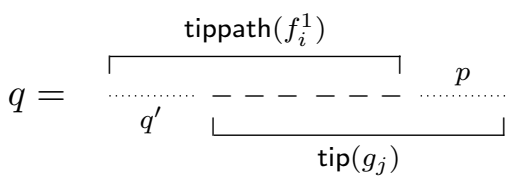

Considerando o elemento $f_{i}^{1} p-\varepsilon_{i *} c q^{\prime} g_{j}$, onde $c=c \ell\left(f_{i}^{1}\right) / c \ell\left(g_{j}\right)$, temos que $\left(f_{i}^{1} p-\varepsilon_{i *} c q^{\prime} g_{j}\right) \mathrm{t}(p)=$ $f_{i}^{1} p-\varepsilon_{i *} c q^{\prime} g_{j}$, ou seja, $f_{i}^{1} p-\varepsilon_{i *} c q^{\prime} g_{j}$ é uniforme à direita. Além disso, como $c q^{\prime} g_{j}=f_{i^{*}}^{0} r \in I$ para algum $r \in R$ e $f_{i^{*}}^{0} r=f_{i^{*}}^{0}\left(f_{i^{*}}^{0} r\right)$, segue que $\varepsilon_{i *} c q^{\prime} g_{j} \in \bigoplus_{l \in T_{0}} f_{l}^{0} I$ e sua (única) coordenada não nula, $c q^{\prime} g_{j}$, está na componente $i^{*}=\operatorname{tipcoord}\left(f_{i}^{1}\right)$. Logo, denotando por $\mu$ o morfismo $\varphi \circ \pi: \bigoplus_{l \in T_{0}} f_{l}^{0} R \rightarrow$ $M$ da apresentação 4.2 , teremos que $\mu\left(f_{i}^{1} p-\varepsilon_{i *} c q^{\prime} g_{j}\right)=\mu\left(f_{i}^{1} p\right)-\mu\left(\varepsilon_{i *} c q^{\prime} g_{j}\right)=0$, uma vez que $\bigoplus_{l \in T_{0}} f_{l}^{0} I:=$ Nuc $\pi \subseteq$ Nuc $\mu$ e que $f_{i}^{1} p \in \bigoplus_{i \in T_{1}} f_{i}^{1} R \subseteq$ Nuc $\mu$. Isto mostra que $f_{i}^{1} p-\varepsilon_{i *} c q^{\prime} g_{j}$ pertence a $\left(\bigoplus_{i \in T_{1}} f_{i}^{1} R\right) \oplus\left(\bigoplus_{i \in U_{1}} f_{i}^{1^{\prime}} R\right)$. Assim, existem únicos $r_{j}$ 's e $s_{l}$ 's em $R$ tais que

$$
\text { (*) } f_{i}^{1} p-\varepsilon_{i *} c q^{\prime} g_{j}=\sum_{j \in T_{1}} f_{j}^{1} r_{j}+\sum_{l \in U_{1}} f_{l}^{1^{\prime}} s_{l} .
$$


Finalmente, definimos o elemento $f_{s}^{2}$ como sendo a diferença $f_{i}^{1} p-\sum_{j \in T_{1}} f_{j}^{1} r_{j}$. Observe que no processo de obtenção da igualdade (*) é importante que o conjunto $\left\{f_{i}^{1}\right\}_{i \in T_{1}} \cup\left\{f_{j}^{1^{\prime}}\right\}_{j \in U_{1}}$ seja tipreduzido à direita.

Antes de definir o conjunto $U_{2}$, apresentamos o lema seguinte que mostra algumas propriedades básicas do elemento $f_{s}^{2}$.

Lema 4.15 Para cada $s=(i, q) \in T_{2}$, o elemento $f_{s}^{2}$ como construído acima possui as seguintes propriedades:

1. é uniforme à direita.

2. pertence ao conjunto $\left(\bigoplus_{i \in T_{1}} f_{i}^{1} R\right) \cap\left(\bigoplus_{j \in T_{0}} f_{j}^{0} I\right)$.

3. $\operatorname{tip}\left(f_{s}^{2}\right)=\operatorname{tip}\left(f_{i}^{1}\right) p$.

Prova.

1. Pela unicidade da igualdade $(*)$ e pelo fato $\left(f_{i}^{1} p-\varepsilon_{i *} c q^{\prime} g_{j}\right) \mathrm{t}(p)=f_{i}^{1} p-\varepsilon_{i *} c q^{\prime} g_{j}$, temos que $f_{j}^{1} r_{j} \mathrm{t}(p)=f_{j}^{1} r_{j}$ e $f_{l}^{1^{\prime}} r_{l} \mathrm{t}(p)=f_{l}^{1^{\prime}} r_{l}$, para todo $j \in T_{1}$ e para todo $l \in U_{1}$. Logo,

$$
f_{s}^{2} \mathrm{t}(p)=\left(f_{i}^{1} p-\sum_{j \in T_{1}} f_{j}^{1} r_{j}\right) \mathrm{t}(p)=f_{i}^{1} p-\sum_{j \in T_{1}} f_{j}^{1} r_{j}=f_{s}^{2} .
$$

2. Por hipótese cada $f_{i}^{1^{\prime}} \in \bigoplus_{l \in T_{0}} f_{l}^{0} I$ e, como observado anteriormente, $\varepsilon_{i *} c q^{\prime} g_{j} \in \bigoplus_{l \in T_{0}} f_{l}^{0} I$. Desse modo, o resultado segue das seguintes igualdades:

$$
f_{s}^{2}:=f_{i}^{1} p-\sum_{j \in T_{1}} f_{j}^{1} r_{j}=\varepsilon_{i *} c q^{\prime} g_{j}+\sum_{l \in U_{1}} f_{l}^{1^{\prime}} s_{l}
$$

3. Como $\left\{f_{l}^{1}\right\}_{l \in T_{1}} \cup\left\{f_{l}^{1^{\prime}}\right\}_{l \in U_{1}}$ é um base Gröbner (à direita) tip-reduzida à direita para $\left(\bigoplus_{i \in T_{1}} f_{i}^{1} R\right) \oplus$ $\left(\bigoplus_{i \in U_{1}} f_{i}^{1^{\prime}} R\right)$, e que $\operatorname{tip}\left(f_{i}^{1} p-\varepsilon_{i *} c q^{\prime} g_{j}\right) \prec \operatorname{tip}\left(f_{i}^{1} p\right)$, então $\operatorname{tip}\left(f_{i}^{1} p\right) \succ \operatorname{tip}\left(f_{j}^{1} r_{j}\right)$, para todo $j \in T_{1}$. Além disso, da parte 1 . sabemos que $f_{s}^{2} \mathrm{t}(p)=f_{s}^{2}$ (uniforme à direita) e, portanto, tip $\left(f_{s}^{2}\right)=$ $\operatorname{tip}\left(f_{i}^{1} p\right)=\operatorname{tip}\left(f_{i}^{1}\right) p$.

Passamos a definir $U_{2}$, o conjunto de índices para os $f_{j}^{2^{\prime}}$ 's, e em seguida construir o $f_{s}^{2^{\prime}}$, para $\operatorname{algum} s \in U_{2}$ fixado.

- $U_{2}:=\left\{(i, q): i \in T_{1}\right.$ e $\left.q \in N\left(\operatorname{tippath}\left(f_{i}^{1}\right)\right)\right\}$

Assim como $T_{2}$, é claro que $U_{2}$ é um conjunto enumerável. Se $s=(i, q) \in U_{2}$, então pela definição de $N$ existem um caminho $z \in \mathcal{B}$ e um $g_{j} \in \mathcal{G}$ tais que $q=\operatorname{tippath}\left(f_{i}^{1}\right) z \operatorname{tip}\left(g_{j}\right)$.

$$
q=\stackrel{\operatorname{tippath}\left(f_{i}^{1}\right)}{\square-----\ldots \ldots \ldots \ldots . .------} \underset{\operatorname{tip}\left(g_{j}\right)}{\underbrace{}_{----D}}
$$


Definimos então $f_{s}^{2^{\prime}}:=f_{i}^{1} z g_{j}$. Dessa forma, temos que $f_{s}^{2^{\prime}} \in\left(\bigoplus_{i \in T_{1}} f_{i}^{1} R\right) \oplus\left(\bigoplus_{i \in U_{1}} f_{i}^{1^{\prime}} R\right)$ e que $\operatorname{tip}\left(f_{s}^{2^{\prime}}\right)=\operatorname{tip}\left(f_{i}^{1}\right) z \operatorname{tip}\left(g_{j}\right)$. Além disso, não é difícil ver que $f_{s}^{2^{\prime}} \in\left(\bigoplus_{i \in T_{1}} f_{i}^{1} R\right) \cap\left(\bigoplus_{j \in T_{0}} f_{j}^{0} I\right)$.

O próximo teorema mostra as principais propriedades $\operatorname{dos} f_{i}^{2}$ 's e $\operatorname{dos} f_{i}^{2^{\prime}}$ 's e estas propriedades nos permitirão construir uma $\Lambda$-resolução projetiva para $M_{\Lambda}$.

\section{Teorema 4.16}

$$
\left(\bigoplus_{i \in T_{1}} f_{i}^{1} R\right) \bigcap\left(\bigoplus_{j \in T_{0}} f_{j}^{0} I\right)=\left(\bigoplus_{l \in T_{2}} f_{l}^{2} R\right) \bigoplus\left(\bigoplus_{l \in U_{2}} f_{l}^{2^{\prime}} R\right)
$$

$e$

$$
\left\{f_{l}^{2}\right\}_{l \in T_{2}} \cup\left\{f_{l}^{2^{\prime}}\right\}_{l \in U_{2}}
$$

é um conjunto uniforme à direita e tip-reduzido e, portanto, uma base de Gröbner à direita tipreduzida (formada por elementos uniformes à direita) para $\left(\bigoplus_{i \in T_{1}} f_{i}^{1} R\right) \cap\left(\bigoplus_{j \in T_{0}} f_{j}^{0} I\right)$. Além disso, cada $f_{s}^{2^{\prime}} \in \bigoplus_{j \in T_{1}} f_{j}^{1} I$.

Prova.

Observemos que $f_{s}^{2^{\prime}}=f_{s}^{2^{\prime}} \mathrm{t}\left(\operatorname{tip}\left(g_{j}\right)\right)$. Assim, pelo primeiro item do Lema 4.15, segue que $\left\{f_{l}^{2}\right\}_{l \in T_{2}} \cup\left\{f_{l}^{2^{\prime}}\right\}_{l \in U_{2}}$ é um conjunto uniforme à direita. Como $g_{j} \in I$, concluímos que cada $f_{s}^{2^{\prime}}:=\left(f_{i}^{1} z\right) g_{j} \in \bigoplus_{j \in T_{1}} f_{j}^{1} I$.

Antes de verificar que o conjunto $\left\{f_{l}^{2}\right\}_{l \in T_{2}} \cup\left\{f_{l}^{2^{\prime}}\right\}_{l \in U_{2}}$ é tip-reduzido à direita, faremos algumas observações sobre os tip's dos $f_{l}^{2}$ 's e $\operatorname{dos} f_{l}^{2^{\prime}}$ 's:

- sabemos que se $s=(i, q) \in T_{2}$ e $g_{j}$ é a relação final do caminho $q$, então existem caminhos $p$ e $q^{\prime}$ tais que $\operatorname{tippath}\left(f_{i}^{1}\right) p=q^{\prime} \operatorname{tip}\left(g_{j}\right)$. Além disso vimos que $\operatorname{tip}\left(f_{s}^{2}\right)=\operatorname{tip}\left(f_{i}^{1}\right) p$, de onde segue que tippath $\left(f_{s}^{2}\right)=\operatorname{tippath}\left(f_{i}^{1}\right) p$. Assim, se $i^{*}=\operatorname{tipcoord}\left(f_{i}^{1}\right)$, então $\operatorname{tippath}\left(f_{i}^{1}\right) p$ aparece na $i^{*}$-ésima componente de $f_{s}^{2}$, visto como elemento de $\bigoplus_{l \in T_{0}} f_{l}^{0} R$.

- por outro lado, se $s=(i, q) \in U_{2}$ e $g_{j}$ é a relação final do caminho $q$, então existe um caminho $z$ tal que $q=\operatorname{tippath}\left(f_{i}^{1}\right) z \operatorname{tip}\left(g_{j}\right)$. Como $f_{s}^{2^{\prime}}:=f_{i}^{1} z g_{j}$, então temos que $\operatorname{tippath}\left(f_{s}^{2^{\prime}}\right)=$ $\operatorname{tippath}\left(f_{i}^{1} z g_{j}\right)=\operatorname{tippath}\left(f_{i}^{1} z\right) \operatorname{tip}\left(g_{j}\right)=\operatorname{tippath}\left(f_{i}^{1}\right) z \operatorname{tip}\left(g_{j}\right)$, onde a segunda e a terceira igualdade são obtidas do Lema 4.8, respectivamente. Logo, se $i^{*}=\operatorname{tipcoord}\left(f_{i}^{1}\right)$, então $\operatorname{tippath}\left(f_{s}^{2^{\prime}}\right)=\operatorname{tippath}\left(f_{i}^{1}\right) z \operatorname{tip}\left(g_{j}\right)$ ocorre na $i^{*}$-ésima de $f_{s}^{2^{\prime}}$ como elemento de $\bigoplus_{l \in T_{0}} f_{l}^{0} R$.

A partir destas observações, podemos provar que $\left\{f_{l}^{2}\right\}_{l \in T_{2}} \cup\left\{f_{l}^{2^{\prime}}\right\}_{l \in U_{2}}$ é um conjunto tipreduzido. Mostraremos inicialmente que cada um dos conjuntos $\left\{f_{l}^{2}\right\}_{l \in T_{2}}$ e $\left\{f_{l}^{2^{\prime}}\right\}_{l \in U_{2}}$ é tip-reduzido à direita. De fato, se existirem $s=(i, q), t=\left(j, q^{\prime}\right) \in T_{2}$ tais que $s \neq t$ e $\left.\operatorname{tip}\left(f_{s}^{2}\right)\right|_{e} \operatorname{tip}\left(f_{t}^{2}\right)$, então $\operatorname{tipcoord}\left(f_{s}^{2}\right)=\operatorname{tipcoord}\left(f_{t}^{2}\right)$ e $\left.\operatorname{tippath}\left(f_{s}^{2}\right)\right|_{e} \operatorname{tippath}\left(f_{t}^{2}\right)$. Mas dado que $\operatorname{tippath}\left(f_{s}^{2}\right)=\operatorname{tippath}\left(f_{i}^{1}\right) p_{i}$ e tippath $\left(f_{t}^{2}\right)=\operatorname{tippath}\left(f_{j}^{1}\right) p_{j}, \operatorname{teremos}$ que tipcoord $\left(f_{i}^{1}\right)=\operatorname{tipcoord}\left(f_{j}^{1}\right)$ e

$$
\operatorname{tippath}\left(f_{j}^{1}\right) p_{j}=\operatorname{tippath}\left(f_{t}^{2}\right)=\operatorname{tippath}\left(f_{s}^{2}\right) p=\operatorname{tippath}\left(f_{i}^{1}\right) p_{i} p,
$$


ou seja, $\left.\operatorname{tip}\left(f_{j}^{1}\right)\right|_{e} \operatorname{tip}\left(f_{i}^{1}\right)$ ou $\left.\operatorname{tip}\left(f_{i}^{1}\right)\right|_{e} \operatorname{tip}\left(f_{j}^{1}\right)$. Neste caso, dado que o conjunto $\left\{f_{l}^{1}\right\}_{l \in T_{i}}$ é tipreduzido, concluímos que $i=j$. Assim, temos uma das seguinte situações:

$$
\begin{aligned}
& q^{\prime}=q w: \quad \frac{\operatorname{tippath}\left(f_{i}^{1}\right) \quad \operatorname{tip}\left(g_{l}\right)}{\underbrace{--------\ldots \ldots \ldots}_{\operatorname{tip}\left(g_{k}\right)}}
\end{aligned}
$$

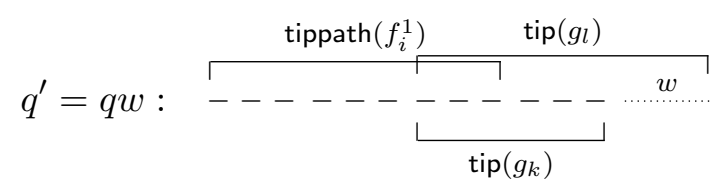

onde, no caso 4.5 temos uma contradição com a condição (3) da definição de $X\left(\operatorname{tippath}\left(f_{i}^{1}\right)\right)$, e no caso 4.6 temos uma contradição com o fato do conjunto $\left\{g_{j}\right\}_{j}$ ser tip-reduzido. Tais contradições se devem a suposição de que o conjunto $\left\{f_{l}^{2}\right\}_{l \in T_{2}}$ não é tip-reduzido. De forma semelhante, verifica-se que $\left\{f_{l}^{2^{\prime}}\right\}_{l \in U_{2}}$ é tip-reduzido.

Resta-nos verificar que não existem $s \in T_{2}$ e $s^{\prime} \in U_{2}$ tais que $\left.\operatorname{tip}\left(f_{s}^{2}\right)\right|_{e} \operatorname{tip}\left(f_{s^{\prime}}^{2^{\prime}}\right)$ ou $\left.\operatorname{tip}\left(f_{s^{\prime}}^{2^{\prime}}\right)\right|_{e} \operatorname{tip}\left(f_{s}^{2}\right)$. Suponhamos que existam $s=(i, q) \in T_{2}$ e algum $s^{\prime}=\left(i^{\prime}, q^{\prime}\right) \in U_{2}, \operatorname{tipcoord}\left(f_{s}^{2}\right)=\operatorname{tipcoord}\left(f_{s^{\prime}}^{2^{\prime}}\right)$ e $\left.\operatorname{tippath}\left(f_{s}^{2}\right)\right|_{e} \operatorname{tippath}\left(f_{s^{\prime}}^{2^{\prime}}\right)$ ou $\left.\operatorname{tippath}\left(f_{s^{\prime}}^{2^{\prime}}\right)\right|_{e} \operatorname{tippath}\left(f_{s}^{2}\right)$.

Se $\left.\operatorname{tippath}\left(f_{s}^{2}\right)\right|_{e} \operatorname{tippath}\left(f_{s^{\prime}}^{2^{\prime}}\right)$ então

$$
\operatorname{tippath}\left(f_{i^{\prime}}^{1}\right) z \operatorname{tip}\left(g_{j}\right)=\operatorname{tippath}\left(f_{s^{\prime}}^{2^{\prime}}\right)=\operatorname{tippath}\left(f_{s}^{2}\right) w=\operatorname{tippath}\left(f_{i}^{1}\right) p w
$$

e, portanto, tippath $\left(f_{i^{\prime}}^{1}\right)$ divide tippath $\left(f_{i}^{1}\right)$ à esquerda, ou vice-versa. Em qualquer dos casos, dado que $\left\{f_{l}^{1}\right\}_{l \in T_{i}}$ é tip-reduzido, concluímos que $i=i^{\prime}$. Mas então, $\operatorname{como} \operatorname{tippath}\left(f_{s}^{2}\right) \operatorname{divide} \operatorname{tippath}\left(f_{s^{\prime}}^{2^{\prime}}\right)$ à esquerda, concluímos que a relação final de $q$ aparece antes da relação final de $q^{\prime}$, o que contradidiz a condição (3) da definição de $X\left(\operatorname{tippath}\left(f_{i}^{1}\right)\right)$ :

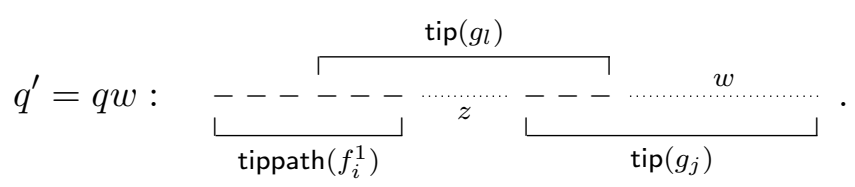

De forma análoga, prova-se que $\operatorname{tippath}\left(f_{s^{\prime}}^{2^{\prime}}\right)$ não divide $\operatorname{tippath}\left(f_{s}^{2}\right)$ à esquerda.

Mostramos assim que o conjunto $\left\{f_{l}^{2}\right\}_{l \in T_{2}} \cup\left\{f_{l}^{2^{\prime}}\right\}_{l \in U_{2}}$ é tip-reduzido. Para concluir a prova do teorema, vamos mostrar a igualdade 4.4.

Agora, dado que o conjunto $\left\{f_{l}^{2}\right\}_{l \in T_{2}} \cup\left\{f_{l}^{2^{\prime}}\right\}_{l \in U_{2}}$ é tip-reduzido uniforme à direita, temos que o submódulo gerado por este conjunto pode ser escrito como $\left(\bigoplus_{l \in T_{2}} f_{l}^{2} R\right) \oplus\left(\bigoplus_{l \in U_{2}} f_{l}^{2^{\prime}} R\right)$ (pelo Lema 4.11). Sendo assim, mostraremos que o conjunto $\left\{f_{l}^{2}\right\}_{l \in T_{2}} \cup\left\{f_{l}^{2^{\prime}}\right\}_{l \in U_{2}}$ gera $\left(\bigoplus_{i \in T_{1}} f_{i}^{1} R\right) \cap$ 
$\left(\bigoplus_{j \in T_{0}} f_{j}^{0} I\right)$. Porém, a inclusão

$$
\left(\bigoplus_{l \in T_{2}} f_{l}^{2} R\right) \bigoplus\left(\bigoplus_{l \in U_{2}} f_{l}^{2^{\prime}} R\right) \subseteq\left(\bigoplus_{i \in T_{1}} f_{i}^{1} R\right) \bigcap\left(\bigoplus_{j \in T_{0}} f_{j}^{0} I\right)
$$

já foi observada anteriormente.

Suponhamos que $\left\{f_{l}^{2}\right\}_{l \in T_{2}} \cup\left\{f_{l}^{2^{\prime}}\right\}_{l \in U_{2}}$ não gera $\left(\bigoplus_{i \in T_{1}} f_{i}^{1} R\right) \cap\left(\bigoplus_{j \in T_{0}} f_{j}^{0} I\right)$ e seja $x \in\left(\bigoplus_{i \in T_{1}} f_{i}^{1} R\right) \cap$ $\left(\bigoplus_{j \in T_{0}} f_{j}^{0} I\right)$ tal que tip $(x)$ é minimal (com respeito a ordem $\succ$ ) com a propriedade

$$
x \notin\left(\bigoplus_{l \in T_{2}} f_{l}^{2} R\right) \bigoplus\left(\bigoplus_{l \in U_{2}} f_{l}^{2^{\prime}} R\right)
$$

Dado que $x \in \bigoplus_{i \in T_{1}} f_{i}^{1} R$ e os $f_{i}^{1}$ 's são tip-reduzidos, então $\operatorname{tip}(x)=\operatorname{tip}\left(f_{i}^{1} p\right)=\operatorname{tip}\left(f_{i}^{1}\right) p$, para algum $i \in T_{1}$ e algum $p \in \mathcal{B}$. Por outro lado, como $x$ também pertence a $\bigoplus_{j \in T_{0}} f_{j}^{0} I$, temos que existem $q, z \in \mathcal{B}$ e $j \in T_{0}$ tais que tip $(x)=\varepsilon_{i^{*}} q \operatorname{tip}\left(g_{j}\right) z$, pois $\left\{g_{j}\right\}_{j}$ é base de Gröbner tip-reduzida para $I$. Então, ou $\operatorname{tip}\left(g_{j}\right)$ sobrepõe $\operatorname{tippath}\left(f_{i}^{1}\right)$ ou não.

Se eles se sobrepõem, então existe $l \in T_{2}$ tal que $\operatorname{tip}\left(f_{l}^{2}\right) z=\operatorname{tip}(x)$, isto pela definição de $T_{2}$ e pelo fato que podemos escolher $j$ de forma que $q$ tenha comprimento mínimo com a propriedade $\operatorname{tip}(x)=\varepsilon_{i^{*}} q \operatorname{tip}\left(g_{j}\right) z$. Como $\operatorname{tip}\left(x-c f_{l}^{2} z\right) \prec \operatorname{tip}(x)$, onde $c=\mathrm{c} \ell(x) / \mathrm{c} \ell\left(f_{l}^{2}\right)$, segue que a diferença $x-c f_{l}^{2} z$ é um elemento de $\left(\bigoplus_{l \in T_{2}} f_{l}^{2} R\right) \oplus\left(\bigoplus_{l \in U_{2}} f_{l}^{2^{\prime}} R\right)$, o que contradiz 4.7 .

Se eles não se soprepõem, então existem $w \in \mathcal{B}$ e $l \in U_{2}$ tais que $\operatorname{tip}\left(f_{l}^{2^{\prime}}\right) w=\operatorname{tip}(x)$. No entanto, dado que tip $\left(x-c f_{l}^{2^{\prime}} w\right) \prec \operatorname{tip}(x)$, onde $c=c \ell(x) / c \ell\left(f_{l}^{2^{\prime}}\right)$, segue que $x-c f_{l}^{2^{\prime}} w \in\left(\bigoplus_{l \in T_{2}} f_{l}^{2} R\right) \oplus$ $\left(\bigoplus_{l \in U_{2}} f_{l}^{2^{\prime}} R\right)$, contradizendo novamente 4.7 .

Como ilustração do procedimento acima, temos o seguinte exemplo:

Exemplo 4.8 Sejam $\Gamma$ o carcás

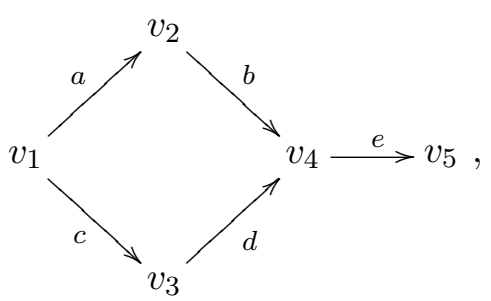

I o ideal de $\mathrm{k} \Gamma$ gerado pelo conjunto $\{a b-c d, b e\}$ e $M=v_{1}\left(\frac{\Lambda}{\underline{\underline{ }}}\right)$, onde $\Lambda=\mathrm{k} \Gamma / I$ e $\underline{\mathrm{r}}$ é o radical 
de Jacobson de $\Lambda$. Então temos o seguinte diagrama

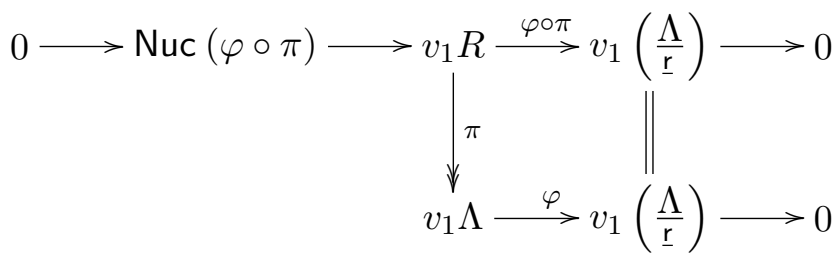

onde $\varphi$ e $\pi$ são projeções canônicas e, denotando por $J$ o ideal das flechas de $\Gamma$, Nuc $(\varphi \circ \pi)=$ $v_{1} J=a R \oplus c R$. De modo que existe uma apresentação

$$
0 \longrightarrow a R \oplus c R \stackrel{H^{1}}{\longrightarrow} v_{1} R \longrightarrow v_{1}\left(\frac{\Lambda}{\underline{r}}\right) \longrightarrow 0
$$

onde $H^{1}(a)=a$ e $H^{1}(c)=c$.

Sejam $f_{1}^{0}=v_{1}, f_{1}^{1}=a$ e $f_{2}^{1}=c$. Neste caso, vemos que $T_{0}=\{1\}$ e $T_{1}=\{1,2\}$. Queremos encontrar os conjuntos $T_{2}:=\left\{(i, q): i \in T_{1}\right.$ e $q \in O\left(\operatorname{tippath}\left(f_{i}^{1}\right)\right\}$ e $U_{2}:=\left\{(i, q): i \in T_{1}\right.$ e $q \in$ $N\left(\operatorname{tippath}\left(f_{i}^{1}\right)\right\}$, e os elementos $f_{s}^{2}$ e $f_{s}^{2^{\prime}}$.

(a) Do Exemplo 4.3, se escolhemos $>_{1}$ como a ordem grau-lexicográfica induzida por $a>\ldots>$ $e>v_{1}>\ldots>v_{5}$, então $\mathcal{G}=\left\{g_{1}=a b-c d, g_{2}=b e, g_{3}=c d e\right\}$ é uma base Gröbner para $o$ ideal I e $\operatorname{tip}(\mathcal{G})=\{a b, b e, c d e\}$.

Observemos que como $\operatorname{tippath}\left(f_{1}^{1}\right)=\operatorname{tip}\left(f_{1}^{1}\right)=a$ e $\operatorname{tippath}\left(f_{2}^{1}\right)=\operatorname{tip}\left(f_{2}^{1}\right)=c$ então $X\left(\operatorname{tippath}\left(f_{1}^{1}\right)\right)=$ $X(a)$ e $X\left(\operatorname{tippath}\left(f_{2}^{1}\right)\right)=X(c)$. Pela parte (a) do Exemplo 4.7 sabemos que $X(a)=\{a b\}=$ $O(a), X(c)=\{c d e\}=O(c)$ e $N(a)=\emptyset=N(c)$. Assim $T_{2}=\{(1, a b),(2, c d e)\}$ e $U_{2}=\emptyset$.

Agora encontraremos os elementos $f_{(1, a b)}^{2}$ e $f_{(2, c d e)}^{2}$. Primeiro note que para $(1, a b)$ temos

$$
f_{1}^{1} p-\varepsilon_{1 *} c q^{\prime} g_{j}=f_{1}^{1} b-v_{1} g_{1}=a b-v_{1}(a b-c d)=c d=f_{2}^{1} d .
$$

Logo $f_{(1, a b)}^{2}:=f_{1}^{1} b-f_{2}^{1} d=a b-c d$. Por outro lado, para $(2, c d e)$ temos

$$
f_{2}^{1} p-\varepsilon_{1 *} c q^{\prime} g_{j}=f_{2}^{1} d e-v_{1} g_{3}=c d e-c d e=0,
$$

de $\operatorname{modo}$ que $f_{(2, c d e)}^{2}:=f_{2}^{1} p-0=f_{2}^{1} d e=c d e$.

Podemos concluir que existe um morfismo de R-módulos

$$
f_{(1, a b)}^{2} R \oplus f_{(2, c d e)}^{2} R \stackrel{H^{2}}{\longrightarrow} f_{1}^{1} R \oplus f_{2}^{1} R
$$

definido por $H^{2}\left(f_{(1, a b)}^{2}\right)=f_{1}^{1} b-f_{2}^{1} d$ e por $H^{2}\left(f_{(2, c d e)}^{2}\right)=f_{2}^{1} d e$.

(b) Se escolhemos $>_{2}$, então $\mathcal{G}=\{a b-c d$,be $\}$ é uma base Gröbner e tip $(\mathcal{G})=\{c d$, be $\}$. Pela parte (b) do exemplo anterior sabemos que $X(a)=\{a b e\}=N(a), X(c)=\{c d\}=O(c)$ e 
$O(a)=\emptyset=N(c)$. Assim, $T_{2}=\{(2, c d)\}$ e $U_{2}=\{(1, a b e)\}$. Além disso,

$$
f_{2}^{1} d-v_{1} g_{1}=c d-(a b-c d)=2 c d-a b
$$

de modo que $f_{(2, c d)}^{2}=f_{2}^{1} d-(2 c d-a b)=a b-c d$. Por outro lado, como $q:=$ abe $=$ $\operatorname{tippath}\left(f_{1}^{1}\right) v_{2} \operatorname{tip}\left(g_{2}\right)$, então

$$
f_{(1, a b e)}^{2^{\prime}}:=f_{1}^{1} v_{2} g_{2}=a v_{2} b e=a b e
$$

Finalmente, temos uma aplicação

$$
f_{(1, a b e)}^{2^{\prime}} R \oplus f_{(2, c d)}^{2} R \stackrel{H^{2}}{\longrightarrow} f_{1}^{1} R \oplus f_{2}^{1} R
$$

dada por $H^{2}\left(f_{(1, a b e)}^{2^{\prime}}\right)=f_{1}^{1} b e$ e $H^{2}\left(f_{(2, c d)}^{2}\right)=f_{1}^{1} b-f_{2}^{1} d$.

Para concluir a parte principal da nossa construção precisamos apenas mostrar a existência da apresentação 4.3. Para isto, necessitamos do lema abaixo:

Lema 4.17 Sejam $L, M_{1}, M_{2}$ e $N$ módulos sobre um anel $A$ tais que existe uma sequência exata da forma

$$
0 \longrightarrow L \longrightarrow M_{1} \oplus M_{2} \stackrel{g}{\longrightarrow} N \longrightarrow 0
$$

onde $M_{2} \subseteq \operatorname{Nuc}(g)$. Então existe um diagrama comutativo

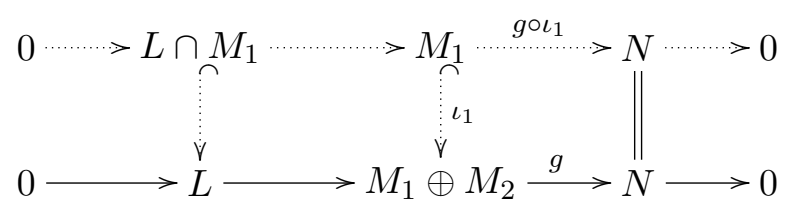

onde $\iota_{1}: M_{1} \rightarrow M_{1} \oplus M_{2}$ é a inclusão na primeira coordenada e $L \cap M_{1}=\operatorname{Nuc}\left(g \circ \iota_{1}\right)$. 
Afirmação: Do Lema 4.17, da igualdade $\left(\bigoplus_{i \in T_{1}} f_{i}^{1} R\right) \cap\left(\bigoplus_{j \in T_{0}} f_{j}^{0} I\right) \stackrel{4.4}{=}\left(\bigoplus_{l \in T_{2}} f_{l}^{2} R\right) \oplus\left(\bigoplus_{l \in U_{2}} f_{l}^{2^{\prime}} R\right)$ (Teorema 4.16) e do diagrama comutativo

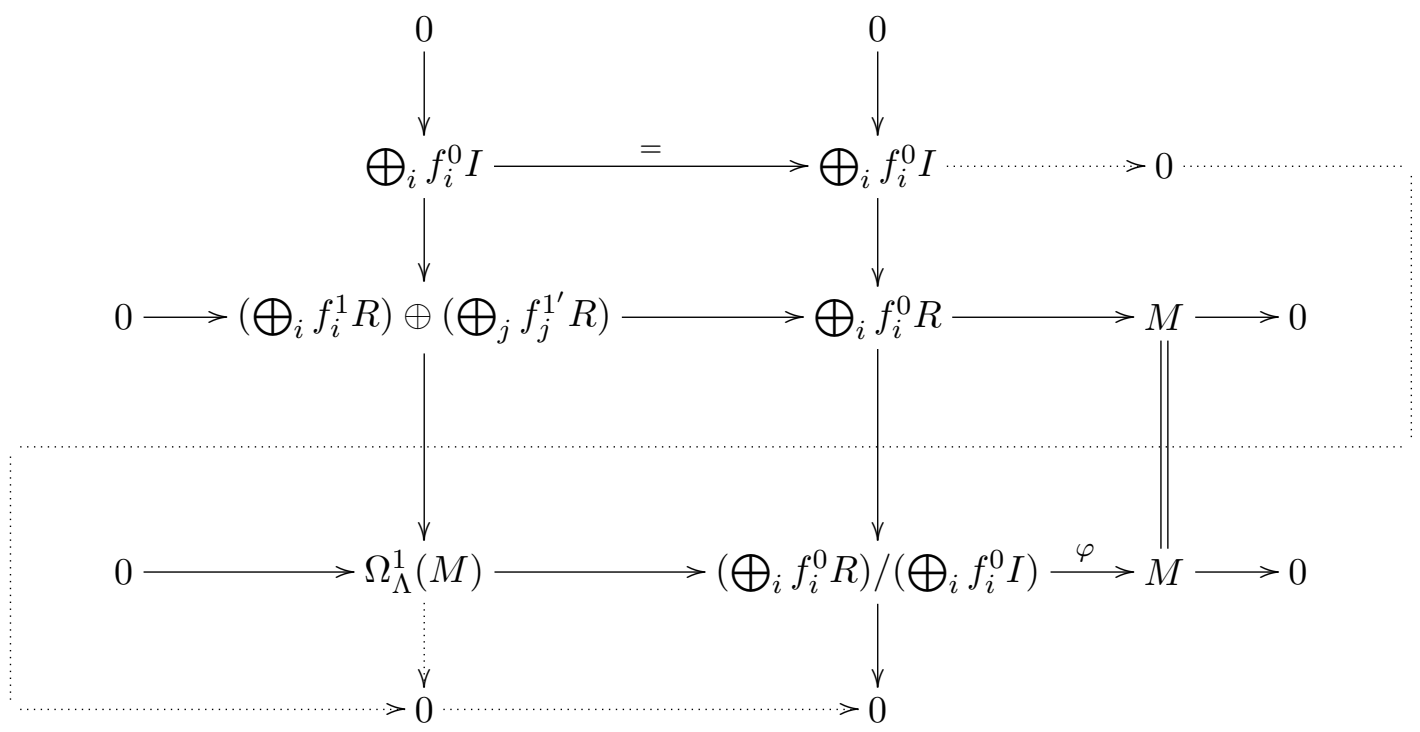

(que pode ser obtido completando o diagrama 4.1) concluímos que existe a sequência

$$
0 \longrightarrow\left(\bigoplus_{i \in T_{2}} f_{i}^{2} R\right) \oplus\left(\bigoplus_{j \in U_{2}} f_{j}^{2^{\prime}} R\right) \stackrel{H^{2}}{\longrightarrow} \bigoplus_{i \in T_{1}} f_{i}^{1} R \longrightarrow \Omega_{\Lambda}^{1}(M) \longrightarrow 0
$$

onde $H^{2}$ é uma inclusão.

$\triangleright$ De fato, considerando a sequência exata

$$
0 \longrightarrow \bigoplus_{i \in T_{0}} f_{i}^{0} I \longrightarrow\left(\bigoplus_{i \in T_{1}} f_{i}^{1} R\right) \oplus\left(\bigoplus_{j \in U_{1}} f_{j}^{1^{\prime}} R\right) \longrightarrow \Omega_{\Lambda}^{1}(M) \longrightarrow 0
$$

(coluna do lado esquerdo no diagrama acima) e observando que $\bigoplus_{j \in U_{1}} f_{j}^{1^{\prime}} R$ está contido no núcleo do epimorfismo $\left(\bigoplus_{i \in T_{1}} f_{i}^{1} R\right) \oplus\left(\bigoplus_{j \in U_{1}} f_{j}^{1^{\prime}} R\right) \rightarrow \Omega_{\Lambda}^{1}(M)$, do Lema 4.17 obtemos a sequência

$$
0 \longrightarrow\left(\bigoplus_{i \in T_{0}} f_{i}^{0} I\right) \cap\left(\bigoplus_{i \in T_{1}} f_{i}^{1} R\right) \longrightarrow \bigoplus_{i \in T_{1}} f_{i}^{1} R \longrightarrow \Omega_{\Lambda}^{1}(M) \longrightarrow 0
$$

A afirmação segue da igualdade $\left(\bigoplus_{j \in T_{0}} f_{j}^{0} I\right) \cap\left(\bigoplus_{i \in T_{1}} f_{i}^{1} R\right) \stackrel{4.4}{=}\left(\bigoplus_{l \in T_{2}} f_{l}^{2} R\right) \oplus\left(\bigoplus_{l \in U_{2}} f_{l}^{2^{\prime}} R\right)$.

Agora estamos aptos a construir uma resolução projetiva de um módulo sobre $\Lambda=R / I$. Para tanto, suponhamos que $M$ é um $\Lambda$-módulo à direita de modo que quando visto como um $R$-módulo possui uma apresentação ${ }^{4}$ projetiva da forma

$$
0 \longrightarrow\left(\bigoplus_{i \in T_{1}} f_{i}^{1} R\right) \oplus\left(\bigoplus_{j \in U_{1}} f_{j}^{1^{\prime}} R\right) \stackrel{H^{1}}{\longrightarrow} \bigoplus_{i \in T_{0}} f_{i}^{0} R \stackrel{\mu}{\longrightarrow} M \longrightarrow 0
$$

\footnotetext{
${ }^{4}$ Como observado anteriormente, tal apresentação sempre existe.
} 
onde $H^{1}$ é uma inclusão, os $f_{i}^{0}$ 's, $f_{i}^{1}$ 's e $f_{i}^{1^{\prime}}$ 's são elementos uniformes à direita, cada $f_{j}^{1^{\prime}}$ é um elemento de $\bigoplus_{i \in T_{0}} f_{i}^{0} I$, o conjunto $\left\{f_{i}^{1}\right\}_{i \in T_{1}} \cup\left\{f_{j}^{1^{\prime}}\right\}_{j \in U_{1}}$ é tip-reduzido e $T_{1}$ é um conjunto enumerável. Com isto, podemos construir um conjunto de elementos uniformes à direita, $\left\{f_{i}^{2}\right\}_{i \in T_{2}} \cup\left\{f_{j}^{2^{\prime}}\right\}_{j \in U_{2}}$, tip-reduzido de modo que

$$
\left(\bigoplus_{j \in T_{0}} f_{j}^{0} I\right) \cap\left(\bigoplus_{i \in T_{1}} f_{i}^{1} R\right) \stackrel{4.4}{=}\left(\bigoplus_{l \in T_{2}} f_{l}^{2} R\right) \oplus\left(\bigoplus_{l \in U_{2}} f_{l}^{2^{\prime}} R\right)
$$

e

$$
0 \longrightarrow\left(\bigoplus_{l \in T_{2}} f_{l}^{2} R\right) \oplus\left(\bigoplus_{l \in U_{2}} f_{l}^{2^{\prime}} R\right) \stackrel{H^{2}}{\longrightarrow} \bigoplus_{i \in T_{1}} f_{i}^{1} R \longrightarrow \Omega_{\Lambda}^{1}(M) \longrightarrow 0
$$

é uma sequência exata entre $R$-módulos (à direita), onde $H^{2}$ é uma inclusão e

$$
\Omega_{\Lambda}^{1}(M)=\operatorname{Nuc}\left(\varphi: \bigoplus_{j \in T_{0}} f_{j}^{0} R / \bigoplus_{j \in T_{0}} f_{j}^{0} I \longrightarrow M\right)
$$

Substituindo $M$ por $\Omega_{\Lambda}^{1}(M)$ e vendo os $f_{i}^{1}$ 's como os $f_{i}^{0}$ 's, os $f_{i}^{2}$ 's como os $f_{i}^{1}$ 's e os $f_{i}^{2^{\prime}}$ 's como os $f_{i}^{1^{\prime}}$ 's, podemos aplicar o Teorema 4.16 e nossa construção à apresentação 4.9 para construir elementos $f_{i}^{3}$ 's e $f_{i}^{3^{\prime}}$ 's em $\bigoplus_{i \in T_{2}} f_{i}^{2} R$ tais que

$$
\left(\bigoplus_{j \in T_{1}} f_{j}^{1} I\right) \cap\left(\bigoplus_{i \in T_{2}} f_{i}^{2} R\right)=\left(\bigoplus_{l \in T_{3}} f_{l}^{3} R\right) \oplus\left(\bigoplus_{l \in U_{3}} f_{l}^{3^{\prime}} R\right)
$$

e

$$
0 \longrightarrow\left(\bigoplus_{l \in T_{3}} f_{l}^{3} R\right) \oplus\left(\bigoplus_{l \in U_{3}} f_{l}^{3^{\prime}} R\right) \stackrel{H^{3}}{\longrightarrow} \bigoplus_{i \in T_{2}} f_{i}^{2} R \longrightarrow \Omega_{\Lambda}^{2}(M) \longrightarrow 0
$$

é uma sequência exata de $R$-módulos, onde (i) $H^{3}$ é uma inclusão, (ii) os elementos $f_{i}^{3}$ 's e $f_{i}^{3^{\prime}}$ 's são uniformes à direita, (iii) cada $f_{j}^{3^{\prime}}$ está em $\bigoplus_{i \in T_{2}} f_{i}^{2} I$, (iv) o conjunto $\left\{f_{i}^{3}\right\}_{i \in T_{3}} \cup\left\{f_{j}^{3^{\prime}}\right\}_{j \in U_{3}}$ é tip-reduzido, e (v) $\Omega_{\Lambda}^{2}(M)$ é o núcleo da aplicação $\bigoplus_{j \in T_{1}} f_{j}^{1} R / \bigoplus_{j \in T_{1}} f_{j}^{1} I \longrightarrow \Omega_{\Lambda}^{1}(M)$ induzida do epimorfismo $\bigoplus_{j \in T_{1}} f_{j}^{1} R \longrightarrow \Omega_{\Lambda}^{1}(M)$ obtido na sequência 4.9 .

Observação 4.4 Talvez seja interessante verificar que a aplicação $\bar{\psi}: \bigoplus_{j \in T_{1}} f_{j}^{1} R / \bigoplus_{j \in T_{1}} f_{j}^{1} I \longrightarrow$ $\Omega_{\Lambda}^{1}(M)$ acima, induzida pelo epimorfismo $\psi: \bigoplus_{j \in T_{1}} f_{j}^{1} R \longrightarrow \Omega_{\Lambda}^{1}(M)$, é uma função. Para isto, consideremos $x+\left(\bigoplus_{j \in T_{1}} f_{j}^{1} I\right) \in \bigoplus_{j \in T_{1}} f_{j}^{1} R / \bigoplus_{j \in T_{1}} f_{j}^{1} I$ e definamos $\bar{\psi}\left(x+\left(\bigoplus_{j \in T_{1}} f_{j}^{1} I\right)\right):=\psi(x)$. Sejam $x, y \in \bigoplus_{j \in T_{1}} f_{j}^{1} R$ tais que $x-y \in \bigoplus_{j \in T_{1}} f_{j}^{1} I$. Como cada $f_{j}^{1} \in \bigoplus_{i \in T_{0}} f_{i}^{0} R$, segue que $f_{j}^{1} a \in \bigoplus_{i \in T_{0}} f_{i}^{0} I$, para todo $a \in I$. Logo, $\bigoplus_{j \in T_{1}} f_{j}^{1} I \subseteq \bigoplus_{i \in T_{0}} f_{i}^{0} I$ e, portanto, $x-y \in\left(\bigoplus_{j \in T_{0}} f_{j}^{0} I\right) \cap$ $\left(\bigoplus_{i \in T_{1}} f_{i}^{1} R\right)=\operatorname{Nuc}(\psi)$. Isto mostra que $\bar{\psi}$ é uma função. Além disso, não é difícil mostrar que $\bar{\psi}$ é um morfismo de $\Lambda$-módulos. Finalmente, a sobrejetividade de $\bar{\psi}$ é uma consequência direta de sua definição e do fato de $\psi$ ser um epimorfismo.

Segue do diagrama 4.8 que essas aplicações são induzidas pela inclusão $\bigoplus_{i} f_{i}^{1} R \subseteq \bigoplus_{i} f_{i}^{0} R$. 
De um modo geral, repetindo o procedimento acima obteremos, para $n \geq 2$, elementos $f_{i}^{n}$ 's e $f_{i}^{n^{\prime}}$ 's tais que

$$
\left(\bigoplus_{j \in T_{n-2}} f_{j}^{n-2} I\right) \bigcap\left(\bigoplus_{i \in T_{n-1}} f_{i}^{n-1} R\right)=\left(\bigoplus_{l \in T_{n}} f_{l}^{n} R\right) \bigoplus\left(\bigoplus_{l \in U_{n}} f_{l}^{n^{\prime}} R\right)
$$

e

$$
0 \longrightarrow\left(\bigoplus_{l \in T_{n}} f_{l}^{n} R\right) \oplus\left(\bigoplus_{l \in U_{n}} f_{l}^{n^{\prime}} R\right) \stackrel{H^{n}}{\longrightarrow} \bigoplus_{i \in T_{n-1}} f_{i}^{n-1} R \longrightarrow \Omega_{\Lambda}^{n-1}(M) \longrightarrow 0
$$

é uma sequência exata de $R$-módulos com $\Omega_{\Lambda}^{n-1}(M)$ sendo o núcleo da aplicação

$$
\bigoplus_{j \in T_{n-2}} f_{j}^{n-2} R / \bigoplus_{j \in T_{n-2}} f_{j}^{n-2} I \longrightarrow \Omega_{\Lambda}^{n-2}(M)
$$

e

(i) $H^{n}$ é uma inclusão.

(ii) Os elementos $f_{i}^{n}$ 's e $f_{i}^{n^{\prime}}$ 's são uniformes à direita.

(iii) Cada $f_{i}^{n^{\prime}}$ pertence a $\bigoplus_{j \in T_{n-2}} f_{j}^{n-1} I$.

(iv) O conjunto $\left\{f_{i}^{n}\right\}_{i \in T_{n}} \cup\left\{f_{i}^{n^{\prime}}\right\}_{i \in U_{n}}$ é tip-reduzido.

Além disso, para cada $f_{i}^{n} \in \bigoplus_{j \in T_{n-1}} f_{j}^{n-1} R$, existem elementos em $R$, digamos $h_{l, i}^{n-1, n}$, tais que

$$
f_{i}^{n}=\sum_{l \in T_{n-1}} f_{l}^{n-1} h_{l, i}^{n-1, n}
$$

Finalmente, seja

$$
P^{n}:=\bigoplus_{j \in T_{n}} f_{j}^{n} R / \bigoplus_{j \in T_{n}} f_{j}^{n} I
$$

e denotando por $v_{i}^{n}$ o vértice de $\Gamma$ tal que $f_{i}^{n}=f_{i}^{n} v_{i}^{n}$, vemos que $P_{n}$ é isomorfo a $\bigoplus_{i \in T_{n}} v_{i}^{n} \Lambda$ (veja Lema 4.4), para todo $n \geq 0$, de modo que cada $P^{n}$ é um $\Lambda$-módulo projetivo. Por outro lado, se $f_{i}^{n+1}=\sum_{l \in T_{n}} f_{l}^{n} h_{l, i}^{n, n+1}$, defina $\delta^{n+1}: P^{n+1} \rightarrow P^{n} \operatorname{como} \delta^{n+1}\left(f_{i}^{n+1}+\bigoplus_{l \in T_{n+1}} f_{l}^{n+1} I\right)$ sendo igual a $f_{j}^{n} h_{j, i}^{n, n+1}+\bigoplus_{l \in T_{n}} f_{l}^{n} I$ na componente de $P^{n}$ correspondente a $f_{j}^{n}+\bigoplus_{l \in T_{n}} f_{l}^{n} I$. Observemos que definida assim, a aplicação $\delta^{n+1}$ pode ser vista como a matriz $\left(\left\{\overline{h_{j, i}^{n, n+1}}\right\}_{i, j}\right)$. Então do Teorema 4.14 concluímos que

$$
(\mathcal{P}, \delta): \quad \cdots \longrightarrow P^{n} \stackrel{\delta^{n}}{\longrightarrow} P^{n-1} \rightarrow \cdots \rightarrow P^{1} \stackrel{\delta^{1}}{\longrightarrow} P^{0} \stackrel{\varphi}{\longrightarrow} M_{\Lambda} \longrightarrow 0
$$

é uma resolução projetiva do $\Lambda$-módulo $M$. Chamamos a resolução $(\mathcal{P}, \delta)$ da resolução associada a apresentação 4.8 .

Finalizaremos a seção com um exemplo que ilustra a construção. 
Exemplo 4.9 Assim como nos Exemplos 4.3, 4.7 e 4.8 sejam $\Gamma$ o carcás

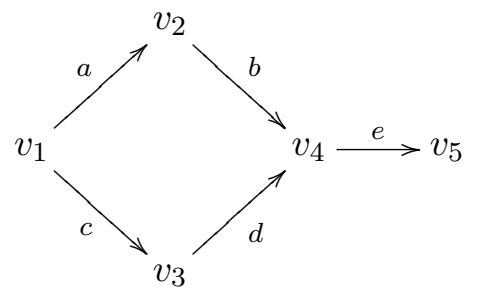

e I o ideal de $\mathrm{k} \Gamma$ gerado pelo conjunto $\{a b-c d, b e\}$. Consideremos $f_{i}^{0}=v_{1}, f_{1}^{1}=a, f_{2}^{1}=c$, $f_{1}^{2}=f_{1}^{1} b-f_{2}^{1} d, f_{2}^{2}=f_{2}^{1}$ de e $>_{1}$ a ordem grau-lexicográfica induzida de $v_{5}<\ldots<v_{1}<e<\ldots<a$. Inicialmente, encontraremos os $f_{i}^{3}$ 's. Como sabemos do Exemplo 4.8, $T_{2}=\{(1, a b),(2$, cde $)\}$, mas para "enxugar" a notação escreveremos apenas $T_{2}=\{1,2\}$. Como $b>d$ e $f_{2}^{2}=f_{1}^{1} 0+f_{2}^{1} d e$, então $\operatorname{tip}\left(f_{1}^{2}\right)=(b, 0)$ e $\operatorname{tip}\left(f_{2}^{2}\right)=(0, d e)$ em $f_{1}^{1} R \oplus f_{2}^{1} R$. Assim $X\left(\operatorname{tippath}\left(f_{1}^{2}\right)\right)=X(b)=\{b e\}^{5} e$ $X\left(\operatorname{tippath}\left(f_{2}^{2}\right)\right)=X(d e)=\emptyset$ (pois o único elemento de $\mathcal{B}$ que divide de à esquerda é o próprio de e ninguém do conjunto tip $(\mathcal{G})=\{a c, b e, c d e\}$ divide de à direita). Logo, $T_{3}=\{(1, b e)\}$ e $U_{3}=\emptyset$.

Agora vamos calcular $f_{(1, b e)}^{3}$. Para tanto, observemos que

$$
f_{1}^{2} e-f_{1}^{1} g_{2}=f_{1}^{1} b e-f_{2}^{1} d e-f_{1}^{1} b e=-f_{2}^{1} d e=-f_{2}^{2},
$$

e segue que $f_{1}^{3}:=f_{(1, b e)}^{3}=f_{1}^{2} e+f_{2}^{2}$. Portanto, temos uma aplicação $H^{3}: f_{1}^{3} \rightarrow f_{1}^{2} R \oplus f_{2}^{2} R$ dada por $H^{3}\left(f_{1}^{3}\right)=\left(e, v_{5}\right)$.

Afirmamos que $T_{4}=\emptyset=U_{4}$. De fato, como e $>v_{5}$, então tip $\left(f_{1}^{3}\right)=(e, 0)$ em $f_{1}^{2} R \oplus f_{2}^{2} R$. Assim, $X\left(\operatorname{tippath}\left(f_{1}^{3}\right)\right)=X(e)=\emptyset$ e com isso $T_{4}=\emptyset=U_{4}$.

Finalmente, para exibir a resolução projetiva de $M_{\Lambda}$ obtida a partir da nossa construção, faremos um resumo dos exemplos anteriores:

- $O$ morfismo $H^{1}: f_{1}^{1} R \oplus f_{2}^{1} R \rightarrow f_{1}^{0} R$ é dado por $H^{1}(a)=a$ e $H^{1}(c)=$ c. Além disso, $f_{1}^{1} R / f_{1}^{1} I \cong v_{2} \Lambda$ e $f_{2}^{1} R / f_{2}^{1} I \cong v_{3} \Lambda$.

- $O$ morfismo $H^{2}: f_{1}^{2} R \oplus f_{2}^{2} R \rightarrow f_{1}^{1} R \oplus f_{2}^{1} R$ é dado por $H^{2}\left(f_{1}^{2}\right)=f_{1}^{1} b-f_{2}^{1} d$ e $H^{2}\left(f_{2}^{2}\right)=f_{2}^{1} d e$. Além disso, $f_{1}^{2} R / f_{1}^{2} I \cong v_{4} \Lambda$ e $f_{2}^{2} R / f_{2}^{2} I \cong v_{5} \Lambda$.

Portanto, a resolução projetiva de $M:=v_{1}(\Lambda / \underline{r})$ encontrada a partir da nossa construção anterior é dada por

$$
0 \longrightarrow v_{5} \Lambda \stackrel{\delta^{3}}{\longrightarrow} v_{4} \Lambda \oplus v_{5} \Lambda \stackrel{\delta^{2}}{\longrightarrow} v_{2} \Lambda \oplus v_{3} \Lambda \stackrel{\delta^{1}}{\longrightarrow} v_{1} \Lambda \longrightarrow M \longrightarrow 0
$$

onde $\delta^{1}=(a c), \delta^{2}=\left(\begin{array}{cc}b & 0 \\ -d & d e\end{array}\right)$ e $\delta^{3}=\left(\begin{array}{c}e \\ v_{5}\end{array}\right)$.

Por outro lado, se considerarmos a ordem $>_{2}$ induzida por $e>\ldots>a>v_{1}>\ldots>v_{5}$, então

\footnotetext{
${ }^{5}$ Exemplo 4.7
} 
$\mathcal{G}=\{a b-c d, b e\}$ é base de Gröbner e $\operatorname{tip}(\mathcal{G})=\{c d, b e\}$. Além disso,

$$
\operatorname{tip}\left(f_{(2, c d)}^{2}\right)=\operatorname{tip}\left(f_{1}^{1} b-f_{2}^{1} d\right)=(0, d)
$$

como elemento de $f_{1}^{1} R \oplus f_{2}^{1} R$. Logo $X\left(\operatorname{tippath}\left(f_{(2, c d)}^{2}\right)=X(d)=\emptyset\right.$ e, portanto, $T_{3}=\emptyset=U_{3}$. De onde segue que a resolução projetiva de $M$, sobre $\Lambda$, construída a partir do nosso procedimento é

$$
0 \longrightarrow v_{4} \Lambda \stackrel{\delta^{2}}{\longrightarrow} v_{2} \Lambda \oplus v_{3} \Lambda \stackrel{\delta^{1}}{\longrightarrow} v_{1} \Lambda \longrightarrow M \longrightarrow 0
$$

onde $\delta^{1}=\left(\begin{array}{lll}a & c\end{array}\right)$ e $\delta^{2}=\left(\begin{array}{c}b \\ -d\end{array}\right)$

Note que a resolução 4.12 é minimal, enquanto a resolução 4.11 não o é. Em particular, podemos concluir que a construção da resolução projetiva de $M_{\Lambda}$ usando nosso método acima, depende da escolha de uma ordem admissível para $\mathcal{B}$. Isso já era esperado, uma vez que tanto a base de Gröbner do ideal $I$ quanto os termos líderes dependem fundamentalmente da escolha da ordem sobre $\mathcal{B}$.

Talvez seja interessante lembrar que em [14] encontramos um método que "ajusta" uma resolução não-minimal a uma resolução minimal. Faremos algo semelhante na seção 4.5.

\subsection{Resoluções finitamente geradas}

Como na maior parte deste capítulo, $\Gamma$ denotará um carcás finito, $\mathrm{k}$ um corpo, $R$ a álgebra de caminhos de $\Gamma$ sobre $\mathrm{k}, \Lambda=R / I$, onde $I$ é um ideal de $R$, e $\mathcal{B}$ a base usual de $R$.

O objetivo dessa seção é encontrar condições suficientes sobre $M$ e $I$ de modo que a resolução $(\mathcal{P}, \delta)$ construída na seção precedente tenha a propriedade adicional que cada termo $P^{n}$ é um $\Lambda$ módulo finitamente gerado. Como veremos, isto dependerá essencialmente da cardinalidade dos conjuntos $T_{0}$ e $T_{1}$ e da escolha de uma ordem admissível para $\mathcal{B}$ sobre a qual o ideal $I$ possui uma base de Gröbner finita.

O próximo resultado mostra que se os conjuntos $T_{0}$ e $T_{1}$ são finitos e existe uma ordem admissível sobre $\mathcal{B}$ de modo que $I$ possui base de Gröbner finita, então a resolução $(\mathcal{P}, \delta)$ é finitamente gerada, ou seja, cada termo dessa resolução é um $\Lambda$-módulo finitamente gerado. Vale salientar que tal resultado não depende do fato do conjunto $U_{1}$ ser finito ou não.

Proposição 4.18 Seja $\Lambda=R / I$, onde $R$ é álgebra de caminhos do carcás $\Gamma$. Suponha que existe uma base multiplicativa ordenada $(\mathcal{B},>)$ sobre a qual existe uma base de Gröbner finita para $I$. Seja $M$ um $\Lambda$-módulo que possui uma apresentação (visto como $R$-módulo)

$$
0 \longrightarrow\left(\bigoplus_{i \in T_{1}} f_{i}^{1} R\right) \oplus\left(\bigoplus_{j \in U_{1}} f_{j}^{1^{\prime}} R\right) \stackrel{H^{1}}{\longrightarrow} \bigoplus_{i \in T_{0}} f_{i}^{0} R \longrightarrow M \longrightarrow 0,
$$

onde $T_{0}$ e $T_{1}$ são conjuntos finitos, $H^{1}$ é uma inclusão, $\left\{f_{i}^{1}\right\}_{i \in T_{1}}$ e $\left\{f_{i}^{1^{\prime}}\right\}_{i \in U_{1}}$ são conjuntos tipreduzidos uniformes à direita e cada $f_{i}^{1^{\prime}}$ é um elemento de $\bigoplus_{i \in T_{0}} f_{i}^{0} I$. Então existe uma resolução 
projetiva $(\mathcal{P}, \delta)$ de $M_{\Lambda}$ (visto como $\Lambda$-módulo ) associada a (*) de modo que cada termo dessa resolução é finitamente gerado.

Prova.

Seja $\mathcal{G}$ uma base de Gröbner finita para $I$ formada por elementos uniformes. Dado que $\mathcal{G}$ é um conjunto finito, então para cada $f_{i}^{1}$ existe apenas um número finito de elementos $g_{j}$ em $\mathcal{G}$ tal que $\operatorname{tippath}\left(f_{i}^{1}\right)$ e tip $\left(g_{j}\right)$ se sobrepõem. Por outro lado, como $T_{1}$ também é um conjunto finito, segue que existe apenas um número finito de $f_{i}^{1}$ 's. Portanto, existe um número finito de $O\left(\operatorname{tippath}\left(f_{i}^{1}\right)\right)$ 's e cada um destes conjuntos é finito. Mas como $T_{2}:=\left\{(i, q): i \in T_{1}\right.$ e $q \in O\left(\operatorname{tippath}\left(f_{i}^{1}\right)\right\}$, concluímos que $T_{2}$ é finito. Por indução, segue que cada $T_{n}, n \geq 0$, é um conjunto finito.

Para concluir a prova, basta lembrar que $P^{n}:=\bigoplus_{i \in T_{n}} f_{i}^{n} R / \bigoplus_{i \in T_{n}} f_{i}^{n} I$, para todo $n \geq 0$.

Nas mesmas hipóteses da proposição acima, note que se for assumido também que o conjunto $U_{1}$ é finito, então $U_{n}$ é finito, para todo $n \geq 1$.

Por [11], temos que se $I$ é um ideal de $R$ tal que $\Lambda=R / I$ é uma álgebra de dimensão finita, então $I$ possui base de Gröbner universal ${ }^{6}$ finita. Tendo em mãos esta observação e a última proposição, seria interessante buscar condições sobre $M_{\Lambda}$ de modo que os conjuntos $T_{0}$ e $T_{1}$ em (*) possam ser escolhidos finitos. E isto é que faremos até o final dessa seção.

A próxima proposição mostra que $T_{0}$ e $T_{1}$ são conjuntos finitos sempre que $M$ for um $R$-módulo finitamente apresentado.

Proposição 4.19 Seja $M$ um $\Lambda$-módulo que visto como $R$-módulo possui uma apresentação finitamente gerada. Suponhamos que existe uma ordem admissivel sobre $\mathcal{B}$ tal que I possui uma base de Gröbner finita com respeito a esta ordem. Então existe uma apresentação da forma 4.8 tal que sua resolução $(\mathcal{P}, \delta)$ associada é finitamente gerada.

Prova.

Sabemos que todo $R$-módulo projetivo é da forma $\bigoplus_{i \in \mathcal{I}} v_{i} R$, onde $\mathcal{I}$ é um conjunto de índices e cada $v_{i}$ é um vértice em $\Gamma$. Como $R$ é uma álgebra hereditária e que $M$ é uma $R$-módulo finitamente apresentado, segue que existe uma apresentação da forma

$$
0 \rightarrow \bigoplus_{i=1}^{n_{1}} w_{i} R \stackrel{\varphi}{\longrightarrow} \bigoplus_{i=1}^{n_{0}} v_{i} R \longrightarrow M \rightarrow 0
$$

onde os $v_{i}$ 's e os $w_{i}$ 's são vértices em $\Gamma$. Seja $h^{i}:=\varphi\left(w_{i}\right)=\varphi\left(w_{i}\right) w_{i} \in \bigoplus_{i=1}^{n_{0}} v_{i} R$, para cada $i=1, \ldots, n_{1}$. Podemos tip-reduzir o conjunto $\left\{h^{1}, \ldots, h^{n_{1}}\right\}^{7}$ e dividir o conjunto resultante em dois conjuntos $\left\{f_{1}^{1}, \ldots, f_{t_{1}}^{1}\right\}$ e $\left\{f_{1}^{1^{\prime}}, \ldots, f_{u_{1}}^{1^{\prime}}\right\}$ tal que cada $f_{j}^{1^{\prime}} \in \bigoplus_{i=1}^{n_{0}} v_{i} I$. Finalmente, denotando

\footnotetext{
${ }^{6}$ Uma base de Gröbner é dita universal se ela é base de Gröbner com respeito a qualquer ordem admissível sobre $\mathcal{B}$.

${ }^{7}$ Veja Proposição 4.9
} 
$v_{i}$ por $f_{i}^{0}$, para cada $i=1, \ldots, n_{0}$, obtemos uma apresentação para $M_{R}$ da forma

$$
0 \longrightarrow\left(\bigoplus_{i \in T_{1}} f_{i}^{1} R\right) \oplus\left(\bigoplus_{j \in U_{1}} f_{j}^{1^{\prime}} R\right) \stackrel{H^{1}}{\longrightarrow} \bigoplus_{i \in T_{0}} f_{i}^{0} R \longrightarrow M \longrightarrow 0
$$

onde os $f_{i}^{0}$ 's, os $f_{i}^{1}$ 's e os $f_{i}^{1^{\prime}}$ 's são elementos uniformes à direita, os conjuntos $T_{0}=\left\{1, \ldots, n_{0}\right\}$ e $T_{1}=\left\{1, \ldots, t_{1}\right\}$ são finitos e o conjunto $\left\{f_{i}^{1}\right\}_{i \in T_{1}} \cup\left\{f_{i}^{1^{\prime}}\right\}_{i \in U_{1}}$ é tip-reduzido e a aplicação $H^{1}$ é a inclusão de $\operatorname{Im} \varphi=\left\langle h^{1}, \ldots, h^{n_{1}}\right\rangle_{R}=\left(\bigoplus_{i \in T_{1}} f_{i}^{1} R\right) \oplus\left(\bigoplus_{j \in U_{1}} f_{j}^{1^{\prime}} R\right)$ em $\bigoplus_{i \in T_{0}} f_{i}^{0} R$. Nestas condições, podemos aplicar a proposição anterior a apresentação acima e, assim, obter nossa tese.

Com este resultado, podemos perguntar agora quais são os $\Lambda$-módulos que são finitamente apresentados como $R$-módulos.

A próxima proposição mostra que todos os $\Lambda$-módulos de dimensão finita são finitamente apresentados quando vistos como $R$-módulos. Para demonstrá-la, precisamos dos próximos três lemas.

Lema 4.20 Seja A uma k-álgebra, $M$ um A-módulo à direita de dimensão finita e Ann $(M)$ o anulador à direita de $M$. Então $A / \operatorname{Ann}(M)$ é uma k-álgebra de dimensão finita.

Prova.

Como $M$ é um k-espaço vetorial de dimensão finita, então $\operatorname{dim}_{\mathrm{k}} \operatorname{End}_{\mathrm{k}}(M)<\infty$. Com isso, basta mostrar que existe uma injeção linear de $A / \operatorname{Ann}(M)$ em $\operatorname{End}_{\mathrm{k}}(M)$. Para tanto, considere a aplicação $f: A / \operatorname{Ann}(M) \rightarrow \operatorname{End}_{\mathrm{k}}(M)$ dada por $f(\bar{r})=-\cdot \bar{r}$, onde $-\cdot \bar{r}: M \rightarrow M$ é a multiplicação por $\bar{r}$. Não é difícil verificar que $f$ é uma função linear. Por outro lado, se $f(\bar{r})=0$, então $m \cdot r=m \cdot \bar{r}=0$, para todo $m \in M$, e assim $r \in \operatorname{Ann}(M)$. Portanto, $\bar{r}=0$ e $f$ é injetora.

Lema 4.21 Sejam $R$ um anel e $f: X \rightarrow Y, g: Y \rightarrow Z, h: X \rightarrow Z$ morfismos de $R$-módulos tais que $f$ é um epimorfismo e $h=g \circ f$. Então existe um epimorfismo do Nuc $(h)$ no Nuc $(g)$. 
Prova.

Do diagrama

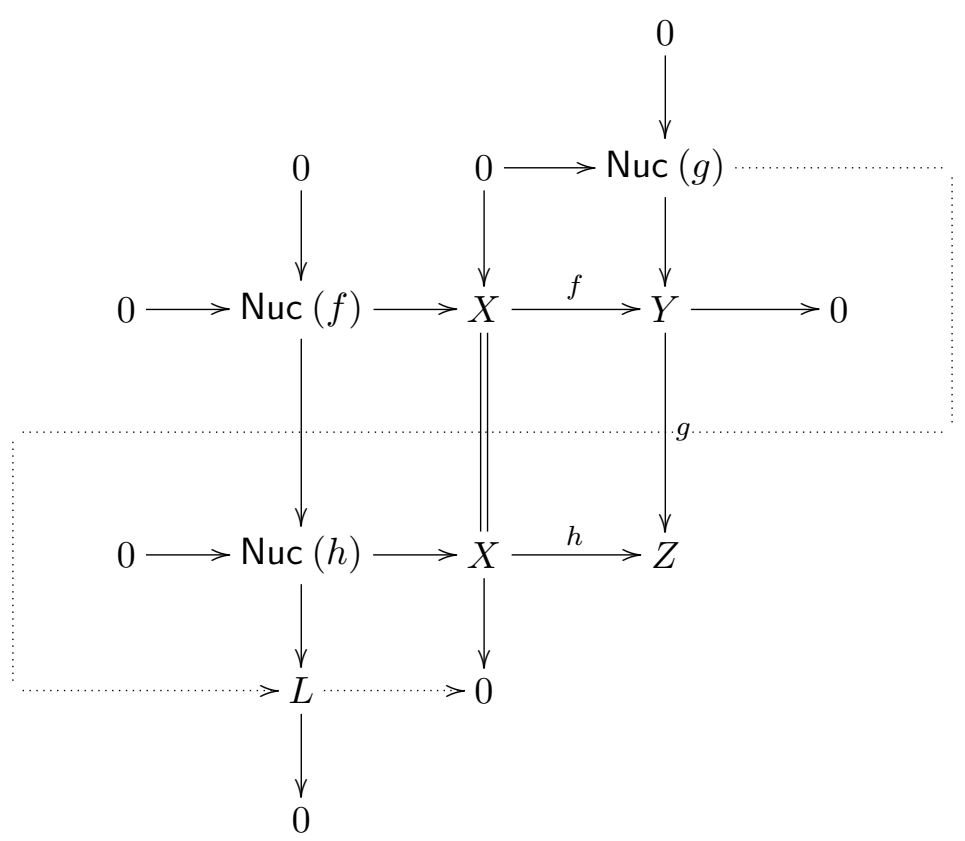

obtemos a sequência exata $0 \rightarrow \operatorname{Nuc}(f) \rightarrow \operatorname{Nuc}(h) \rightarrow L \cong$ Nuc $(g) \rightarrow 0$, e o resultado segue.

Lema 4.22 Sejam $R$ um anel, $X, Y$ e $P$ três $R$-módulos tais que $P$ é um módulo projetivo $e$ existem epimorfismos $p: P \rightarrow Y$ e $\pi: X \rightarrow Y$. Então existe um epimorfismo de $P \oplus \operatorname{Nuc}(\pi)$ em $X$.

Prova.

Dado que $P$ é um módulo projetivo, então existe um morfismo $\varphi: P \rightarrow X$ tal que $p=\pi \circ \varphi$. Agora definamos $\psi: P \oplus \operatorname{Nuc}(\pi) \rightarrow X$ como $\psi(a+t)=\varphi(a)+t$, para todo $a \in P$ e todo $t \in \operatorname{Nuc}(\pi)$. É claro que $\psi$ é um morfismo de $R$-módulos. Resta-nos verificar que $\psi$ é um epimorfismo. De fato, observemos que para cada $x \in X$, existe $a \in P$ tal que $p(a)=\pi(x)$. Assim, como $p=\pi \circ \varphi$, temos que $x-\varphi(a) \in \operatorname{Nuc}(\pi)$. De onde segue que $\psi(a+(x-\varphi(a)):=\varphi(a)+(x-\varphi(a))=x$.

Proposição 4.23 Sejam $\Lambda=R / I$ e $M$ um $\Lambda$-módulo de dimensão finita. Então $M$, visto como $R$-módulo, é finitamente apresentado. Além disso, se I tem base de Gröbner finita, então existe uma apresentação da forma 4.8 com resolução associada $(\mathcal{P}, \delta)$ finitamente gerada.

Prova.

Sejam $\operatorname{Ann}(M)$ o $R$-anulador à direita de $M_{R}$ e $\Sigma=R / \operatorname{Ann}(M)$. Então, pelo Lema 4.20, segue que $\Sigma$ é uma k-álgebra de dimensão finita e, portanto, $M$ é um $\Sigma$-módulo finitamente gerado. Seja $\left\{m_{i}\right\}_{i=1}^{t_{0}}$ um conjunto gerador de $M$ como $\Sigma$-módulo. Dado que cada $m_{i}$ é uma soma de elementos uniformes à direita, então podemos supor, sem perda de generalidade, que o conjunto $\left\{m_{i}\right\}_{i=1}^{t_{0}}$ é 
uniforme à direita. Neste caso, denote por $\left\{f_{i}^{0}\right\}_{i=1}^{t_{0}}$ o conjunto de vértices em $\Gamma$ tal que $m_{i} f_{i}^{0}=m_{i}$, para todo $i=1, \ldots, t_{0}$. Dado que $M$ e $\Sigma$ são de dimensão finita, então existe uma apresentação projetiva de $\Sigma$-módulos da forma

$$
\bigoplus_{i=1}^{d} w_{i} \Sigma \longrightarrow \bigoplus_{i=1}^{t_{0}} f_{i}^{0} \Sigma \stackrel{g}{\longrightarrow} M \rightarrow 0
$$

onde cada $w_{i}$ é um vértice em $\Gamma$. Com isso, obtemos uma sequência exata de $R$-módulos

$$
0 \rightarrow K \longrightarrow \bigoplus_{i=1}^{t_{0}} f_{i}^{0} R \stackrel{\bar{g}}{\longrightarrow} M \rightarrow 0
$$

$\operatorname{com} \bar{g}=g \circ \pi$ - onde $\pi$ é a projeção canônica de $\bigoplus_{i=1}^{t_{0}} f_{i}^{0} R$ em $\bigoplus_{i=1}^{t_{0}} f_{i}^{0} \Sigma$ - e $K=\operatorname{Nuc}(\bar{g})$. Aplicando o Lema 4.21 aos morfismos $g, \pi$ e $\bar{g}$, concluímos que existe um epimorfimo de $K$ em Nuc $(g)$. Por outro lado, do epimorfismo natural de $\bigoplus_{i=1}^{d} w_{i} R$ em Nuc $(g)$, existe o seguinte diagrama:

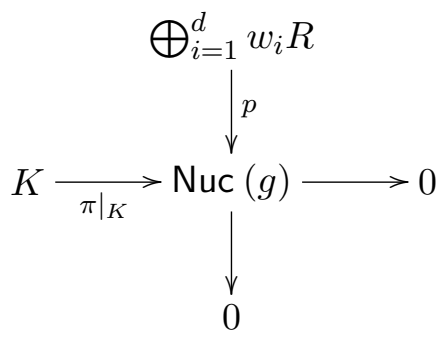

Agora aplicando o Lema 4.22 ao diagrama acima e observando que Nuc $\left(\left.\pi\right|_{K}\right)=\operatorname{Nuc}(\pi)=$ $\bigoplus_{i=1}^{t_{0}} f_{i}^{0} \operatorname{Ann}(M)$, podemos concluir que existe um epimorfismo de $\left(\bigoplus_{i=1}^{d} w_{i} R\right) \oplus\left(\bigoplus_{i=1}^{t_{0}} f_{i}^{0} \operatorname{Ann}(M)\right)$ sobre $K$. Sendo assim, para mostrar que $K$ é um $R$-módulo finitamente gerado, basta verificar que $\bigoplus_{i=1}^{t_{0}} f_{i}^{0} \operatorname{Ann}(M)$ é finitamente gerado. Para tanto, lembremos que o fato de $R / \operatorname{Ann}(M)$ ser uma álgebra de dimensão finita, então $\operatorname{Ann}(M)$ possui uma base de Gröbner finita com respeito a qualquer ordem admissível. Do Exemplo 4.5 segue que $\bigoplus_{i=1}^{t_{0}} f_{i}^{0} \operatorname{Ann}(M)$ é finitamente gerado. Isto conclui a prova de que $M$ é um $R$-módulo finitamente apresentado.

A segunda parte segue da proposição 4.19 .

Da proposição acima, segue que qualquer módulo finitamente gerado $M$ sobre uma álgebra $\Lambda=$ $R / I$ de dimensão finita possui uma resolução projetiva finitamente gerada que pode ser construída a partir do procedimento mostrado na seção anterior. De fato, dado que $\Lambda$ é de dimensão finita, então $M$ é de dimensão finita e $I$ possui uma base de Gröbner finita sobre qualquer ordem admissível.

\subsection{Resoluções de módulos lineares sobre álgebras de Koszul}

Nesta seção modificaremos ${ }^{8}$ a construção da seção 4.3 para produzir resoluções lineares (e portanto, projetivas minimais) de módulos lineares sobre álgebras de Koszul.

\footnotetext{
${ }^{8}$ Como feito em [13].
} 
A menos de menção em contrário, $R=\mathrm{k} \Gamma$ é a álgebra de caminhos de um carcás finito $\Gamma$ sobre um corpo k com a graduação usual, $I \subseteq J^{2}$ um ideal de $R$ graduado, $\Lambda=R / I$ com a graduação induzida de $R$ e $M$ é um $\Lambda$-módulo graduado.

Proposição 4.24 Sejam $\Lambda=R / I$ uma álgebra graduada com $I \subseteq J^{2}$ e $M$ um $\Lambda$-módulo linear. Suponhamos que

$$
\bigoplus_{i=1}^{t_{1}} w_{i} \Lambda \longrightarrow \bigoplus_{i=1}^{t_{0}} v_{i} \Lambda \stackrel{\varphi}{\longrightarrow} M \rightarrow 0
$$

seja um início de uma resolução linear de $M$, onde os $v_{i}$ 's e os $w_{i}$ 's são vértices em $\Gamma$. Então existe uma apresentação projetiva de $M_{R}$ da forma

$$
0 \longrightarrow\left(\bigoplus_{i=1}^{t_{1}} f_{i}^{1} R\right) \oplus\left(\bigoplus_{j \in U_{1}} f_{j}^{1^{\prime}} R\right) \stackrel{H^{1}}{\longrightarrow} \bigoplus_{i=1}^{t_{0}} f_{i}^{0} R \stackrel{\mu}{\longrightarrow} M \longrightarrow 0,
$$

onde os elementos do conjunto $\left\{f_{i}^{0}\right\}_{i=1}^{t_{0}}$ são vértices, os conjuntos $\left\{f_{i}^{1}\right\}_{i=1}^{t_{1}} e\left\{f_{i}^{1^{\prime}}\right\}_{i \in U_{1}}$ são uniformes à direita, tip-reduzidos e $f_{i}^{1}, f_{j}^{1^{\prime}} \in \bigoplus_{i=1}^{t_{0}} f_{i}^{0} R$, para todo $i=1, \ldots, t_{1}$ e para todo $j \in U_{1}$. Além disso, cada $f_{i}^{1}$ pode ser escolhido de modo que como elemento de $\bigoplus_{i=1}^{t_{0}} f_{i}^{0} R$ é uma soma de elementos de comprimento 1 em $R$ e cada $f_{j}^{1^{\prime}}$ pode ser escolhido como um elemento de $\bigoplus_{i=1}^{t_{0}} f_{i}^{0} I$.

Prova.

Da apresentação 4.13, temos o seguinte diagrama

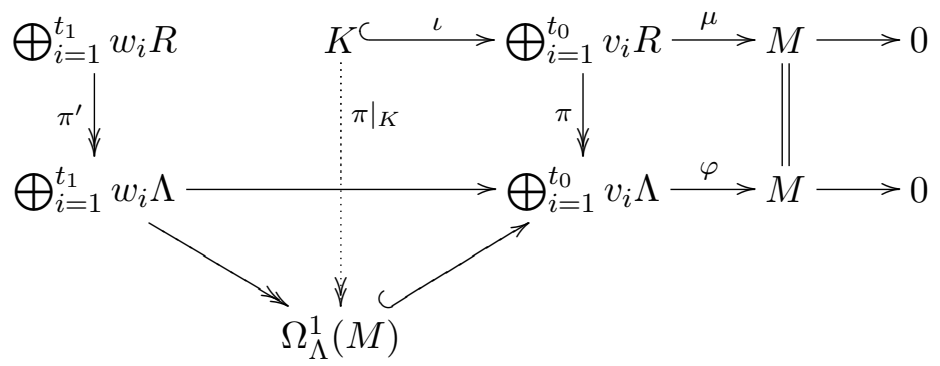

onde o epimorfismo do $R$-módulo projetivo $K:=\operatorname{Nuc}(\mu)$ em Nuc $(\varphi):=\Omega_{\Lambda}^{1}(M)$ é dado pelo Lema 4.21 aplicado aos morfismos $\varphi, \mu$ e $\pi$. Além disso, como a projeção canônica $\pi^{\prime}: \bigoplus_{i=1}^{t_{1}} w_{i} R \rightarrow$ $\bigoplus_{i=1}^{t_{1}} w_{i} \Lambda$ é uma cobertura projetiva na categoria gr $R$ de $R$-módulos graduados (à direita) e morfismos de grau zero, segue que o epimorfismo de $\bigoplus_{i=1}^{t_{1}} w_{i} R$ em $\Omega_{\Lambda}^{1}(M)^{9}$ também é uma cobertura projetiva em $\operatorname{gr} R$. Assim, existe uma sequência em $\operatorname{gr} R$ da forma

$$
0 \longrightarrow \operatorname{Nuc}(\beta) \longrightarrow K \underset{\alpha}{\stackrel{\beta}{\rightleftarrows}} \bigoplus_{i=1}^{t_{1}} w_{i} R \longrightarrow 0
$$

onde $\beta \alpha=\operatorname{id}_{\bigoplus_{i} w_{i} R}$. Em particular, $K$ é isomorfo a $\left(\bigoplus_{i=1}^{t_{1}} w_{i} R\right) \oplus \operatorname{Nuc}(\beta)$.

\footnotetext{
${ }^{9}$ obtido do diagrama acima
} 
Do diagrama comutativo

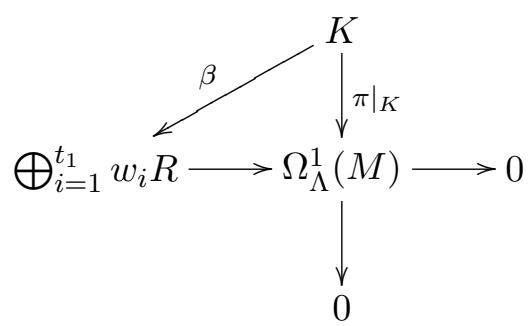

e da igualdade $\operatorname{Nuc}(\pi)=\bigoplus_{i=1}^{t_{0}} v_{i} I$, temos que $\operatorname{Nuc}(\beta) \subseteq \bigoplus_{i=1}^{t_{0}} v_{i} I$. Como Nuc $(\beta)$ é um $R$-módulo projetivo, então existem vértices $\left\{w_{i}^{\prime}\right\}_{i \in U_{1}^{\prime}}$ em $\Gamma_{0}$ tal que Nuc $(\beta) \cong \bigoplus_{i \in U_{1}^{\prime}} w_{i}^{\prime} R$.

Agora sejam

$$
\begin{gathered}
f_{i}^{0}=v_{i}, \text { para todo } i=1, \ldots, t_{0}, \\
h_{i}^{1}=\iota \alpha\left(w_{i}\right), \text { para todo } i=1, \ldots, t_{1}
\end{gathered}
$$

$\mathrm{e}$

$$
h_{i}^{1^{\prime}}=\iota\left(w_{i}^{\prime}\right) \text {, para todo } i \in U_{1}^{\prime} .
$$

Dado que os $w_{i}$ 's e $w_{i}^{\prime}$ 's são vértices em $\Gamma_{0}$, segue que os $h_{i}^{1}$ 's e $h_{i}^{1}$ 's são elementos uniformes à direita. Agora, tip-reduzimos os conjuntos $\left\{h_{i}^{1}\right\}_{i=1}^{t_{1}}$ e $\left\{h_{i}^{1^{\prime}}\right\}_{i \in U_{1}^{\prime}}$ e denotamos o resultado por $\left\{f_{i}^{1}\right\}_{i=1}^{t_{1}}$ e $\left\{f_{i}^{1^{\prime}}\right\}_{i \in U_{1}}$, respectivamente. Lembremos que no processo de tip-redução ${ }^{10}$ a uniformidade (à direita) dos elementos é mantida.

Finalmente, dado que $\alpha$ é uma aplicação de grau zero, $\iota$ é uma inclusão e $M$ é um módulo linear segue que as coordenadas de cada $f_{i}^{1}$, como elemento de $\bigoplus_{i=1}^{t_{0}} f_{i}^{0} R$, são uma soma de elementos de comprimento $1 \mathrm{em} R$. Além disso, como $h_{i}^{1^{\prime}}:=\iota\left(w_{i}^{\prime}\right) \in \operatorname{Nuc}(\beta) \subseteq \bigoplus_{i=1}^{t_{0}} f_{i}^{0} I$ e $f_{l}^{1^{\prime}}=h_{l}^{1^{\prime}}-c h_{j}^{1^{\prime}} p$, para algum $c \in \mathrm{k}$ e algum $p \in \mathcal{B}$, temos que cada $f_{l}^{1^{\prime}}$ pertence a $\bigoplus_{i=1}^{t_{0}} f_{i}^{0} I$ e que cada uma de suas coordenadas é uma soma de elementos em $R$ de comprimento maior ou igual a 2 .

Corolario 4.25 Com a mesma notação da proposição anterior, temos que

$$
\bigoplus_{i=1}^{t_{1}} f_{i}^{1} R / \bigoplus_{i=1}^{t_{1}} f_{i}^{1} I \longrightarrow \Omega_{\Lambda}^{1}(M) \rightarrow 0
$$

é uma cobertura projetiva graduada de $\Omega_{\Lambda}^{1}(M)$, onde $\Omega_{\Lambda}^{1}(M)$ é o núcleo de $\varphi: \bigoplus_{i=1}^{t_{0}} v_{i} \Lambda \longrightarrow M$.

Prova.

Basta observar que tal aplicação é induzida da inclusão $\bigoplus_{i=1}^{t_{1}} f_{i}^{1} R \subseteq \bigoplus_{i=1}^{t_{0}} f_{i}^{0} R$, como já visto na Observação 4.4. Pela proposição anterior, cada $f_{i}^{1}$ quando visto como um elemento de $\bigoplus_{i=1}^{t_{0}} f_{i}^{0} R$ é soma de elementos de comprimento 1 em $R$, ou seja,

$$
f_{i}^{1}=\sum_{j} f_{j}^{0} h_{j, i}
$$

\footnotetext{
${ }^{10}$ Se necessário, veja a Definição 4.11 e a Proposição 4.9.
} 
com cada $h_{j, i}$ em $(\mathrm{k} \Gamma)_{1}$ e o resultado segue da Proposição 3.2.

É importante observar que na prova da Proposição 4.24 não garantimos que a união $\left\{f_{i}^{1}\right\}_{i \in T_{1}} \cup$ $\left\{f_{i}^{1^{\prime}}\right\}_{i \in U_{1}}$ seja um conjunto tip-reduzido pois mostramos apenas que cada um dos conjuntos $\left\{f_{i}^{1}\right\}_{i \in T_{1}}$ e $\left\{f_{i}^{1^{\prime}}\right\}_{i \in U_{1}}$ é tip-reduzido. Mas lembremos que para a construção dos $f_{i}^{2}$ 's a partir da apresentação 4.14 construída na proposição anterior, precisamos que o conjunto $\left\{f_{i}^{1}\right\}_{i \in T_{1}} \cup\left\{f_{i}^{1^{\prime}}\right\}_{i \in U_{1}}$ seja tipreduzido $^{11}$. Veremos na prova do próximo teorema como contornar essa situação.

O conjunto de índices $T_{2}$ para os $f_{s}^{2}$ 's é definido da mesma forma, ou seja, $T_{2}:=\{(i, q): i \in$ $T_{1}$ e $\left.q \in O\left(\operatorname{tippath}\left(f_{i}^{1}\right)\right\}\right)$. Além disso, suponhamos que estejam construídos os $f_{i}^{2}$ 's como na seção 4.3 e que a apresentação resultante seja linear. Neste caso, cada coordenada de $f_{i}^{2}$, como elemento de $\bigoplus_{i=1}^{t_{0}} f_{i}^{0} R$, é uma soma de caminhos de comprimento 2. Se $\Lambda$ for uma álgebra de Koszul, então $I$ é um ideal quadrático e, portanto, existe uma base de Gröbner $\mathcal{G}$ para $I$ tip-reduzida uniforme consistindo de elementos homogêneos de grau maior ou igual a 2. Se na construção do $f_{i}^{2}$ ocorrer um $g_{j} \in \mathcal{G}$ de grau $d$ então o elemento $f_{i}^{2}$ também possui grau $d$. Então se queremos construir uma resolução linear, basta considerar os elementos de grau 2 em $\mathcal{G}$, uma vez que o processo de tip-redução de $\left\{f_{i}^{1}\right\}_{i \in T_{1}} \cup\left\{f_{i}^{1^{\prime}}\right\}_{i \in U_{1}}$ não altera grau. Observe ainda que há somente um número finito desses elementos, pois $\mathcal{G}$ é um conjunto tip-reduzido e em $\Gamma$ existe apenas um número finito de caminhos de comprimento 2.

Teorema 4.26 Sejam $\Lambda=R / I$ uma álgebra de Koszul com $I \subseteq J^{2}$ e $M$ um $\Lambda$-módulo linear que tem uma resolução projetiva linear da forma 4.13. Então existe um procedimento para construir um conjunto finito $\left\{f_{i}^{2}\right\}_{T_{2}}$ de elementos em $\bigoplus_{i=1}^{t_{1}} f_{i}^{1} R$ com $f_{i}^{2}=\sum_{l=1}^{t_{1}} f_{l}^{1} r_{l}$, para alguns elementos homogêneos $r_{l}$ em $R$, tal que

$$
\bigoplus_{i \in T_{2}} f_{i}^{2} R / \bigoplus_{i \in T_{2}} f_{i}^{2} I \stackrel{\delta^{2}}{\longrightarrow} \bigoplus_{i=1}^{t_{1}} f_{i}^{1} R / \bigoplus_{i=1}^{t_{1}} f_{i}^{1} I \longrightarrow \Omega_{\Lambda}^{1}(M) \rightarrow 0
$$

é o início de uma resolução projetiva linear de $\Omega_{\Lambda}^{1}(M)$, onde $\Omega_{\Lambda}^{1}(M)$ é igual ao Nuc $(\varphi)$ e $\delta^{2}$ é a aplicação induzida pela inclusão $\bigoplus_{i \in T_{2}} f_{i}^{2} R \subseteq \bigoplus_{i=1}^{t_{1}} f_{i}^{1} R$.

Prova.

Seja $s=(i, q) \in T_{2}$. Então existe $g_{j} \in \mathcal{G}$ tal que

$$
q=\operatorname{tippath}\left(f_{i}^{1}\right) p=q^{\prime} \operatorname{tip}\left(g_{j}\right)
$$

para alguns caminhos $p$ e $q^{\prime}$ em $\Gamma$. Como observado no parágrafo anterior, precisamos somente daqueles elementos de $\mathcal{G}$ com grau 2. Assim, tip $\left(g_{j}\right)$ é um caminho de comprimento 2 que sobrepõe $\operatorname{tippath}\left(f_{i}^{1}\right)$ e como a proposição anterior garante que as coordenadas de $f_{i}^{1}$ são uma soma de caminhos de comprimento 1 , segue que $p$ é necessariamente uma flecha e $q^{\prime}$ um vértice. Sabemos de nossa construção que o elemento $f_{i}^{1}-\varepsilon_{i^{*}} c q^{\prime} g_{j}$ pertence a $\left(\bigoplus_{i=1}^{t_{1}} f_{i}^{1} R\right) \oplus\left(\bigoplus_{j \in U_{1}} f_{j}^{1^{\prime}} R\right)$, onde

\footnotetext{
${ }^{11}$ Ver seção 4.3
} 
$c=\mathrm{c} \ell\left(f_{i}^{1}\right) / \mathrm{c} \ell\left(g_{j}\right) \in \mathrm{k}$. Mas como o conjunto $\left\{f_{i}^{1}\right\}_{i \in T_{1}} \cup\left\{f_{i}^{1^{\prime}}\right\}_{i \in U_{1}}$ não é necessariamente tipreduzido, então aparentemente não existe um algoritmo que expresse $f_{i}^{1}-\varepsilon_{i} * q^{\prime} g_{j}$ como um elemento da soma (direta) acima.

Afirmação: Quando escrevemos $f_{i}^{1}-\varepsilon_{i^{*}} c q^{\prime} g_{j}$ como uma soma da forma $\sum_{i \in T_{1}} f_{i}^{1} r_{i}+\sum_{j \in U_{1}} f_{j}^{1^{\prime}} s_{j}$, $\operatorname{com} r_{i}, s_{j} \in R$, nenhum $\operatorname{dos} f_{j}^{1^{\prime}}$ 's ocorrendo nessa soma tem grau maior ou igual a três ${ }^{12}$.

$\triangleright$ De fato, se a afirmação fosse falsa, então para cada $f_{l}^{1^{\prime}}$ com grau maior ou igual a 3 existiria um subconjunto $T_{1}^{l}$ de $T_{1}$ de modo que $\sum_{i \in T_{1}^{l}} f_{i} r_{i}=-f_{l}^{1^{\prime}} s_{j}$, pois o grau de $f_{i}^{1}-\varepsilon_{i^{*}} c q^{\prime} g_{j}$ é igual a 2 . E isto seria uma contradição, uma vez que $\left(\bigoplus_{i=1}^{t_{1}} f_{i}^{1} R\right) \cap\left(\bigoplus_{j \in U_{1}} f_{j}^{1^{\prime}} R\right)=\{0\}$

Por outro lado, como existe apenas um número finito de elementos de comprimento 2 em $\Gamma$ e o subconjunto de $\left\{f_{i}^{1^{\prime}}\right\}_{i \in U_{1}}$ formado por elementos homogêneos de grau 2 é ainda um conjunto tip-reduzido, então existe somente um número finito de tais elementos. Denotamos tal subconjunto por $\left\{f_{i}^{1^{\prime}}\right\}_{i \in U_{1(2)}}$, para um conjunto finito $U_{1(2)}$.

Lembremos que na construção ${ }^{13}$ dos elementos $f_{s}^{2}$ 's, assumimos que o conjunto $\left\{f_{i}^{1}\right\}_{i \in T_{1}} \cup$ $\left\{f_{i}^{1^{\prime}}\right\}_{i \in U_{1}}$ é tip-reduzido. Porém, como já chamamos a atenção, a proposição anterior assegura apenas que cada um dos conjuntos $\left\{f_{i}^{1}\right\}_{i \in T_{1}}$ e $\left\{f_{i}^{1^{\prime}}\right\}_{i \in U_{1(2)}}$ é tip-reduzido, mas não necessariamente a união, ou seja, pode ocorrer o caso em que o termo líder de algum $f_{i}^{1}$ divida (à esquerda) o termo líder de algum $f_{j}^{1^{\prime}}$, ou vice-versa. Neste caso, precisamos refinar os conjuntos $\left\{f_{i}^{1}\right\}_{i \in T_{1}}$ e $\left\{f_{i}^{1^{\prime}}\right\}_{i \in U_{1(2)}}$ de modo que a união deste refinamento seja um conjunto tip-reduzido e que, obviamente, suas propriedades principais permaneçam. Para tanto, é interessante fazer a seguinte observação:

Observação: Para o processo de tip-redução de $\left\{f_{i}^{1}\right\}_{i \in T_{1}} \cup\left\{f_{i}^{1^{\prime}}\right\}_{i \in U_{1(2)}}$ podemos nos preocupar somente em "melhorar" os elementos do conjunto $\left\{f_{i}^{1^{\prime}}\right\}_{i \in U_{1(2)}}$, isto é, no resultado da tip-redução o conjunto $\left\{f_{i}^{1}\right\}_{i \in T_{1}}$ não será alterado.

$\triangleright$ De fato, basta verificar que o termo líder de um elemento de $\left\{f_{i}^{1^{\prime}}\right\}_{i \in U_{1(2)}}$ não divide o termo líder de um elemento de $\left\{f_{i}^{1}\right\}_{i \in T_{1}}$. Mas isto ocorre porque as coordenadas de cada $f_{i}^{1}$ têm grau 1 e as coordenadas de cada $f_{j}^{1^{\prime}}$ têm grau $2 \mathrm{e}$, portanto, não é possível que $\left.\operatorname{tippath}\left(f_{j}^{1^{\prime}}\right)\right|_{e} \operatorname{tippath}\left(f_{i}^{1}\right)$, quaisquer que sejam $i \in T_{1}$ e $j \in U_{1(2)}$. De onde segue que não existem $i \in T_{1}$ e $j \in U_{1(2)}$ tais que $\left.\operatorname{tip}\left(f_{j}^{1^{\prime}}\right)\right|_{e} \operatorname{tip}\left(f_{i}^{1}\right)$.

Neste caso, haverá mudanças somente em $\left\{f_{i}^{1^{\prime}}\right\}_{i \in U_{1(2)}}$. Denotaremos os novos elementos por $\left\{\mathbf{f}_{i}^{1^{\prime}}\right\}_{i \in U_{1(2)^{\prime}}}$. É importante lembrar que cada $\mathbf{f}_{l}^{\mathbf{1}^{\prime}}$ é expresso em termos de $f_{j}^{1}$ 's e $f_{j}^{1^{\prime}}$ 's. Além disso, a tip-redução de algum $f_{i}^{1^{\prime}}$ é obtida por subtração de elementos da forma $d f_{i}^{1} a$, onde $a \in \Gamma_{1}$ e $d \in \mathrm{k}$, e elementos da forma $d f_{j}^{1^{\prime}}$, onde $j \in U_{1(2)}$ e $d \in \mathrm{k}$. Portanto, cada $\mathbf{f}_{l}^{1^{\prime}}$ é um elemento homogêneo de grau 2.

Para construir $f_{s}^{2}$, com $s=(i, q) \in T_{2}$, primeiro achamos uma expressão

$$
(*) \quad f_{i}^{1}-\varepsilon_{i^{*}} c q^{\prime} g_{j}=\sum_{l \in T_{1}} f_{l}^{1} r_{l}+\sum_{l \in U_{1(2)^{\prime}}} \mathbf{f}_{l}^{1^{\prime}} s_{l}
$$

para alguns elementos $r_{l}$ e $s_{l}$ em $R$. Como já observado antes, as coordenadas do elemento do

\footnotetext{
${ }^{12}$ quando visto como um elemento de $\bigoplus_{i=1}^{t_{0}} f_{i}^{0} R$

${ }^{13}$ Seção 4.3
} 
lado esquerdo da igualdade acima são uma soma de caminhos de comprimento 2. Se algum caminho de comprimento maior ou igual a 2 ocorre em algum $r_{l}$, então $\operatorname{tippath}\left(\sum_{l \in T_{1}} f_{l}^{1} r_{l}\right)=$ $\operatorname{tippath}\left(\sum_{l \in U_{1(2)}} \mathbf{f}_{l}^{\mathbf{1}^{\prime}} s_{l}\right)$, o que contradiz o fato do conjunto $\left\{f_{i}^{1}\right\}_{i \in T_{1}} \cup\left\{\mathbf{f}_{i}^{\mathbf{1}^{\prime}}\right\}_{i \in U_{1(2)}}$ ser tip-reduzido. Além disso, por argumentos de comprimento, conclui-se que não há vértices ocorrendo em nenhum $r_{l}$. Assim cada $r_{l}$ é uma soma de flechas e cada $s_{l}$ é um vértice. Substituindo cada $\mathbf{f}_{l}^{1^{\prime}}$ por expressões da forma $f_{l}^{1^{\prime}}-c f_{i}^{1} p$, reescrevemos a igualdade $(*)$ na forma

$$
f_{i}^{1}-\varepsilon_{i^{*}} c q^{\prime} g_{j}=\sum_{l \in T_{1}} f_{l}^{1} r_{l}^{\prime}+\sum_{l \in U_{1(2)}} f_{l}^{1^{\prime}} s_{l}^{\prime}
$$

para alguns elementos homogêneos $r_{l}^{\prime}$ e $s_{l}^{\prime}$ de graus 1 e 0 , respectivamente. De modo que

$$
f_{s}^{2}:=f_{i}^{1} p-\sum_{l \in T_{1}} f_{l}^{1} r_{l}^{\prime}=\varepsilon_{i^{*}} c q^{\prime} g_{j}+\sum_{l \in U_{1(2)}} f_{l}^{1^{\prime}} s_{l}^{\prime}
$$

é um elemento homogêneo de grau 2, isto é, quando visto como um elemento de $\bigoplus_{i \in T_{0}} f_{i}^{0} R$ cada uma de suas coordenadas é uma soma de caminhos de comprimento 2.

É importante lembrar que na Seção 4.3 quando mostramos que o conjunto $\left\{f_{i}^{2}\right\}_{i \in T_{2}}$ é tipreduzido usamos apenas o fato de $\left\{f_{i}^{1}\right\}_{i \in T_{1}}$ ser tip-reduzido. Portanto, segue da Proposição 4.24 que $\left\{f_{i}^{2}\right\}_{i \in T_{2}}$ é tip-reduzido. Além disso, é claro que $f_{s}^{2}$ é uniforme à direita.

Pela definição de $f_{s}^{2}$ acima, já sabemos que $\bigoplus_{i \in T_{2}} f_{i}^{2} R \subseteq\left(\bigoplus_{i \in T_{1}} f_{i} R\right) \cap\left(\bigoplus_{i \in T_{0}} f_{i}^{0} I\right)$. Agora mostraremos que os elementos homogêneos de grau $2 \mathrm{em}\left(\bigoplus_{i \in T_{1}} f_{i} R\right) \cap\left(\bigoplus_{i \in T_{0}} f_{i}^{0} I\right)$ pertecencem a $\bigoplus_{i \in T_{2}} f_{i}^{2} R$. Suponha que existe um elemento homogêneo de grau 2 em $\left(\bigoplus_{i \in T_{1}} f_{i} R\right) \cap\left(\bigoplus_{i \in T_{0}} f_{i}^{0} I\right) \backslash$ $\bigoplus_{i \in T_{2}} f_{i}^{2} R$ e denote por $x$ um elemento homogêneo de grau 2 em $\left(\bigoplus_{i \in T_{1}} f_{i} R\right) \cap\left(\bigoplus_{i \in T_{0}} f_{i}^{0} I\right)$, onde tip $(x)$ é o menor possível tal que $x \notin \bigoplus_{i \in T_{2}} f_{i}^{2} R$. Assim $x=\sum_{i=1}^{t_{1}} f_{i}^{1} b_{i}=\sum_{i=1}^{t_{0}} f_{i}^{0} g_{j_{i}}$, onde $b_{i} \in R$ e $g_{j_{i}} \in \mathcal{G}$ são elementos homogêneos de graus 1 e 2, respectivamente. Então existem $i \in T_{1} \mathrm{e}$ $l \in T_{0}$ tais que $\operatorname{tip}(x)=\operatorname{tip}\left(f_{i}^{1} b_{i}\right)=f_{l}^{0} \operatorname{tip}\left(g_{j_{l}}\right)$. Neste caso, temos que $\operatorname{tippath}\left(f_{i}^{1}\right) b_{i}=f_{l}^{0} \operatorname{tip}\left(g_{j_{l}}\right) \mathrm{e}$ tomando $s=\left(i, \operatorname{tippath}\left(f_{i}^{1}\right) b_{i}\right) \in T_{2}$ segue que $\operatorname{tip}(x)=\operatorname{tip}\left(f_{s}^{2}\right)$. Pela minimalidade de $x$ segue que $x-c f_{s}^{2} \in \bigoplus_{i \in T_{2}} f_{i}^{2} R$, onde $\mathrm{c}=\mathrm{c} \ell(x) / \mathrm{c} \ell\left(f_{s}^{2}\right) \in \mathrm{k}$, o que é um absurdo.

Então construímos um conjunto $\left\{f_{i}^{2}\right\}_{i \in T_{2}}$, tip-reduzido uniforme à direita com todos os elementos homogêneos de grau 2, tal que $\left(\bigoplus_{i \in T_{1}} f_{i} R\right) \cap\left(\bigoplus_{i \in T_{0}} f_{i}^{0} I\right) \cap(\mathrm{k} \Gamma)_{2}=\left(\bigoplus_{i \in T_{2}} f_{i}^{2} R\right) \cap(\mathrm{k} \Gamma)_{2}$.

Finalmente, podemos demonstrar que

$$
\bigoplus_{i \in T_{2}} f_{i}^{2} R / \bigoplus_{i \in T_{2}} f_{i}^{2} I \stackrel{\delta^{2}}{\longrightarrow} \bigoplus_{i=1}^{t_{1}} f_{i}^{1} R / \bigoplus_{i=1}^{t_{1}} f_{i}^{1} I \longrightarrow \Omega_{\Lambda}^{1}(M) \rightarrow 0
$$

é linear. Pelo corolário acima, restá-nos provar que $\bigoplus_{i \in T_{2}} f_{i}^{2} R / \bigoplus_{i \in T_{2}} f_{i}^{2} I$ é gerado em grau 2 - o que segue do fato de cada $f_{i}^{2}$ ser homogêneo de grau 2 - e que existe uma aplicação $\bigoplus_{i \in T_{2}} f_{i}^{2} R / \bigoplus_{i \in T_{2}} f_{i}^{2} I \longrightarrow \Omega_{\Lambda}^{2}(M) \rightarrow 0$ de grau 0 . Do diagrama comutativo com linhas e col- 
unas exatas

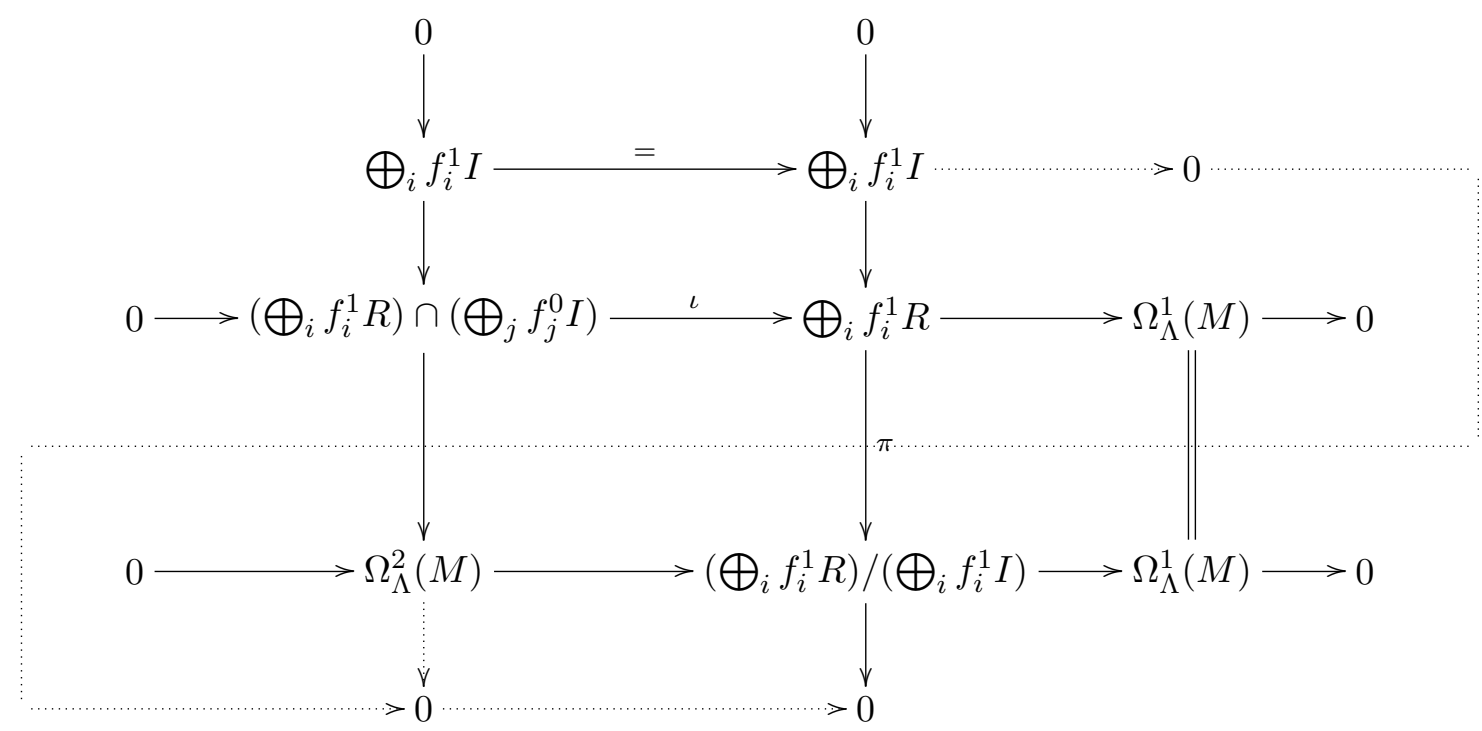

obtemos um epimorfismo $\left(\bigoplus_{i} f_{i}^{1} R\right) \cap\left(\bigoplus_{j} f_{j}^{0} I\right) \rightarrow \Omega_{\Lambda}^{2}(M)$, que é a restrição do epimorfismo canônico $\pi$. Como $\Omega_{\Lambda}^{2}(M)$ é um módulo de Koszul gerado em grau 2, então existe $X$ contido em $\left(\bigoplus_{i} f_{i}^{1} R\right) \cap$ $\left(\bigoplus_{j} f_{j}^{0} I\right)$ gerado em grau 2 tal que $\pi_{\left.\right|_{X}}$ é uma cobertura projetiva minimal graduada de $\Omega_{\Lambda}^{2}(M)$. Por outro lado como $\left(\bigoplus_{i \in T_{1}} f_{i} R\right) \cap\left(\bigoplus_{i \in T_{0}} f_{i}^{0} I\right) \cap(\mathrm{k} \Gamma)_{2}=\left(\bigoplus_{i \in T_{2}} f_{i}^{2} R\right) \cap(\mathrm{k} \Gamma)_{2}$, então $X \subseteq \bigoplus_{i \in T_{2}} f_{i}^{2} R$ e, portanto, $\pi$ restrita a $\bigoplus_{i \in T_{2}} f_{i}^{2} R$ é ainda um epimorfismo. Mas tal aplicação é igual a $\pi \circ \bar{\iota}$, onde $\bar{\iota}$ é a restrição de $\iota$ a $\bigoplus_{i \in T_{2}} f_{i}^{2} R$, ou seja, é uma aplicação induzida pela inclusão $\bigoplus_{i \in T_{2}} f_{i}^{2} R \subseteq \bigoplus_{i} f_{i}^{1} R$. Além disso, como $f_{j}^{2}=\sum_{l} f_{l}^{1} r_{l}$ com $r_{l} \in(\mathrm{k} \Gamma)_{1}$ tal aplicação preserva graus.

Como já observado anteriormente, a construção apresentada na Seção 4.3 não produz necessariamente uma resolução projetiva minimal para um dado módulo (linear). O procedimento apresentado na presente seção difere daquele no sentido que aqui ao construirmos um $f_{s}^{2}$ consideramos somente os elementos da base de Gröbner de grau dois. ${ }^{14}$ Ilustraremos isto com o exemplo seguinte.

Exemplo 4.10 Assim como no Exemplo 4.9 sejam $\Gamma$ o carcás

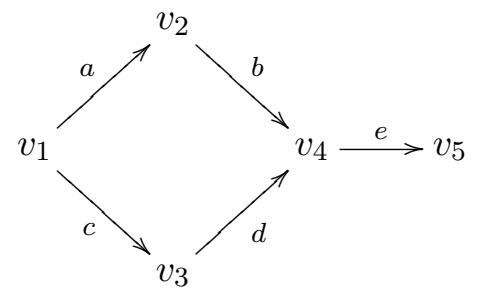

e I o ideal de $\mathrm{k} \Gamma$ gerado pelo conjunto $\{a b-c d, b e\}$. Note que $\Lambda=\mathrm{k} \Gamma / I$ é uma álgebra de Koszul e, portanto, que $M=v_{1}(\Lambda / \underline{r})$ é um módulo linear. Como já observado antes, a resolução projetiva

\footnotetext{
${ }^{14}$ De certa forma, não precisamos de todos os índices do conjunto $T_{2}$, mas somente daqueles que dependem dos elementos de grau 2 da base de Gröbner.
} 
(obtida no Exemplo 4.9 usando-se a ordem $>_{1}$ )

$$
0 \longrightarrow v_{5} \Lambda \longrightarrow v_{4} \Lambda \oplus v_{5} \Lambda \longrightarrow v_{2} \Lambda \oplus v_{3} \Lambda \longrightarrow v_{1} \Lambda \longrightarrow M \longrightarrow 0,
$$

de $M$ não é minimal. Para esta ordem, $\mathcal{G}=\{a b-c d$, be, cde $\}$ é uma base de Gröber para I. Assim, para a construção de um $f_{s}^{2}$, usando o procedimento acima, precisamos somente considerar os elementos em $T_{2}$ que dependam do subconjunto $\{a b-c d, b e\}$, já que "cde" tem grau 3. Desta forma, produzimos somente $f_{(1, a b)}^{2}=a b-c d$. Portanto, assim como no Exemplo 4.9, obtemos a resolução

$$
0 \longrightarrow \frac{f_{1}^{2} R}{f_{1}^{2} I} \longrightarrow \frac{f_{1}^{1} R}{f_{1}^{1} I} \oplus \frac{f_{2}^{1} R}{f_{2}^{1} I} \longrightarrow \frac{v_{1} R}{v_{1} I} \longrightarrow M \longrightarrow 0
$$

projetiva (minimal) linear de $M$. 


\section{Considerações Finais}

A resolução projetiva apresentada no último capítulo pode ser usada para se obter resultados teóricos e computacionais. No trabalho [16], Green e Marcos usaram essa resolução para obter resultados sobre uma nova generalização de álgebras de Koszul, que são as chamadas Álgebras 2-d deteminadas e as álgebras $2-d$ koszul, eles provam que as álgebras 2- $d$ determinadas monomiais têm sua álgebra de extensões finitamente geradas. Eles conjecturam que esse resultado vale em geral. Nós pretendemos em nosso doutorado, estudar essa e outras conjecturas usando a resolução aqui descrita. Pretendemos aprender a utilizar as implementações existentes da resolução aqui descrita.

Outro objeto para estudo futuro é a pergunta deixada no final do Capítulo 2 sobre a existência de álgebras homologicamente auto-duais não monomiais. Achamos que será possível resolver o problema fazendo uso da teoria de bases de Gröbner. 


\section{Referências Bibliográficas}

[1] I. Assem, D. Simson, and A. Skowronski, Elements of the representation theory of associative algebras, vol. 1, USA, 2006.

[2] M. Auslander, I. Reiten, and S. O. Smal $\oslash$, Representation theory of artin algebras, Cambridge Stud. Adv. Math. 36, Cambridge University Press, Cambridge, 1995.

[3] J. Michael Bardzell, Resolutions and cohomology of finite dimensional algebras, Phd. Thesis, Virginia Polytechnic Institute and State University, Virginia, 1996.

[4] _ The alternating syzygy behavior of monomial algebras, J. Algebra 188 (1997), no. 1, $69-89$.

[5] A. A. Villas Bôas, Aspectos algébricos e computacionais de base de Gröbner não comutativas, Dissertação de Mestrado, Universidade de São Paulo, São Paulo, 2005.

[6] R. Bautista, P. Gabriel, A. V. Roiter, and L. Salmeron, Representation-finite algebras and multiplicative bases, Invent. Math. 81 (1985), 217-285.

[7] A. A. Beilinson, V. A. Ginzburg, and W. Soergel, Koszul duality patterns in representation theory, J. Amer. Math. Soc. 9 (1996), no. 2, 473-527.

[8] D. J. Benson, Representations and cohomology 1, Cambridge Stud. Adv. Math. 30, Cambridge University Press, United Kingdom, 1998.

[9] K. Bongartz, Algebras and quadratic forms, J. London Math. Soc. 28 (1983), no. 3, 461-469.

[10] E. Cline, B. Parshall, and L. Scott, Abstract Kazhdan-Lustig theories, Tohoku Math. J. 45 (1993), no. 4, 511-534.

[11] E. L. Green, Noncommutative Gröbner bases, and projective resolutions, Computational methods for representations of groups and algebras (Essen, 1997), Progr. Math. 173 (1999), 29-60.

[12] _ Multiplicative bases, Gröbner bases, and right Gröbner bases, Symbolic computation in algebra, analysis, and geometry (Berkeley, 1998), J. Symbolic Comput. 29353 (2000), no. 4-5, 601-623.

[13] E. L. Green and $\emptyset$. Solberg, An algorithmic approach to resolutions, J. Symbolic Comput. 42 (2007), no. 11-12, 1012-1033.

[14] E. L. Green, $\emptyset$. Solberg, and D. Zacharia, Minimal projective resolutions, Trans. Amer. Math. Soc. 353 (2001), 2915-2939.

[15] E. L. Green, D. Happel, and D. Zacharia, Projective resolutions over artin algebras with zero relations, Illinois Journal of Algebra 29 (1983), no. 1, 80-90. 
[16] E. L. Green and E. N. Marcos, d-Koszul algebras, 2-d determined algebras and 2-d-Koszul algebras, preprint.

[17] E. L. Green, E. N. Marcos, R. Martinez-Villa, and P. Zhang, d-koszul algebras, Journal of Pure and Applied Algebra 193 (2004), 141-163.

[18] E. L. Green and R. Martínez-Villa, Koszul and Yoneda algebras, Representation theory of algebras (Cocoyoc, 1994), CMS Conf. Proc., Amer. Math. Society, Providence, RI 18 (1996), $247-297$.

[19] Koszul and Yoneda algebras II, Representation theory of algebras (Cocoyoc, 1994), CMS Conf. Proc., Amer. Math. Society, Providence, RI 24 (1998), 227-244.

[20] E. L. Green and D. Zacharia, The cohomology ring of a monomial algebra, Manuscripta Math. 85 (1994), 11-23.

[21] S. Mac Lane, Homology, Spring-Verlag: Classics in Mathematics, New York, 1995.

[22] C. Năstăsescu and F. Van Oystaeyen, Graded ring theory, North-Holland Mathematical Library 28, New York, 1982.

[23] B. Parshall and L. Scott, Derived categories, quasi-hereditary algebras and algebraic groups, Mathematical Lecture Notes Series 3 (1988), 1-105.

[24] _ Koszul algebras and the Frobenius automorphism, Quart. J. Math. Oxford 46 (1995), no. $2,345-384$.

[25] S. B. Priddy, Koszul resolutions, Trans. Amer. Math. Soc. 152 (1970), 39-60.

[26] D. Zacharia, Ext and Koszul algebras, notas de mini-curso, seminário conjunto AMS-Sociedad Mexicana de Matematicas, não publicadas (1990). 


\section{Índice Remissivo}

álgebra

de Koszul, 35

de Yoneda, 23

graduada, 16

0,1-split-básica, 19

gerada em graus 0 e 1, 16

homologicamente auto-dual, 29

monomial, 14

quadrática, 14

de caminhos, 13

oposta, 4

base

de Gröbner, 49

à direita, 52

à direita tip-reduzida, 52

reduzida, 50

tip-reduzida, 51

multiplicativa, 48

ordenada, 51

cobertura projetiva graduada, 20

coeficiente líder, 47

conjunto tip-reduzido, 50

à direita, 57

elemento

homogênio, 16, 18

uniforme, 51

ideal graduado, 16

módulo

de Koszul, 35

graduado, 18

de grau $k, 18$

ocorre em, 47

ordem

admissível, 48 grau-lexicográfica, 48

tip-índice, 55

produto de Yoneda, 22

radical de Jacobson graduado, 17

resolução lenear, 33

resolução projetiva graduada, 20

minimal, 20

teorema

da comparação, 10

da adjunção, 12

termo líder, 47, 52, 56 